\author{
UNIVERSITY OF SÃO PAULO \\ SÃO CARLOS SCHOOL OF ENGINEERING
}

GABRIEL SALES CANDIDO SOUZA

EVALUATION OF LAMINATED COMPOSITE PLATES

BEHAVIOR UNDER SHEAR-AFTER-IMPACT

LOADING CONDITIONS: A METHODOLOGY

PROPOSAL

São Carlos

2021 



\section{EVALUATION OF LAMINATED COMPOSITE PLATES BEHAVIOR UNDER SHEAR-AFTER-IMPACT LOADING CONDITIONS: A METHODOLOGY PROPOSAL}

Text submitted for the degree of Master of Sciences presented to the São Carlos School of Engineering of the University of São Paulo in fulfillment of the requirements for the degree of Master in Mechanical Engineering.

Concentration Area: Aeronautical Engineering

Supervisor: Associate Professor Volnei Tita

\section{VERSÃO CORRIGIDA}

São Carlos 


\section{FOLHA DE JULGAMENTO}

Candidato: Engenheiro GABRIEL SALES CANDIDO SOUZA.

Título da dissertação: "Avaliação do comportamento de placas laminadas de material compósito sob cisalhamento após carregamento de impacto".

Data da defesa: 26/02/2021 .

\section{Comissão Julgadora}

Prof. Associado Volnei Tita

(Orientador)

(Escola de Engenharia de São Carlos - EESC/USP)

Prof. Dr. Rui Jorge Costa de Miranda Guedes

(Universidade do Porto/U.PORTO)

Prof. Dr. Maikson Luiz Passaia Tonatto

(Universidade Federal de Santa Maria/UFSM)

Coordenador do Programa de Pós-Graduação em Engenharia Mecânica: Prof. Associado Carlos de Marqui Junior

Presidente da Comissão de Pós-Graduação:

Prof. Titular Murilo Araujo Romero

\section{Resultado}

APROVADO

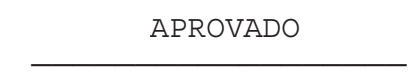



Dedico este trabalho à minha irmã Gabriela

pelo seu sucesso e crescimento na batalha de superação da dor e por nos mostrar que tudo nesta vida é efêmero. 


\section{ACKNOWLEDGEMENTS}

Firstly, I would like to thank my family for all the love, support and comprehension since always. To my parents Mara and Alberto, my grandmothers Martha and Vastir, my brother Tiê and my sisters Gabriela and Maitê, I would like to leave a special thanks; love you all.

To the advisor of this Master's Dissertation, professor Volnei Tita, for being such a reasonable person and the best possible advisor I could have ever dreamed of. Your balance, orientation and friendship were of unique importance for the development of this work and made it a good ride during this time. Thank you a lot, you are an inspiration for me.

To my laboratory friends, for the conversations, help through this work and moments which own a special place in my heart. Also, to my other friends from other places and University of Brasília, that even far away holds great importance in my life. A special thanks to Ciro and Natasha, the best couple ever; uncle Gabriel eagerly waits for Livia's birth. I would like to mention the priceless debt that I owe to Fernando Madureira, Bruno Christoff, Maísa Maciel, Gregório Ferreira, Denys Marques, Marcelo Leite, Matheus Urzedo, Victor Barcelos, Abraão Ferreira, Lucas Santana, Thiago Scheiner, Rommel Lucas and Pedro Senna for being such loyal and supportive friends. Also, if during this period of social isolation I have never truly felt myself alone, I owe this to Renan Prado, Guilherme Lucon, Igor Uenohara, Luiz Gustavo Humphries, Paulo Bancatelli and Arthur Almeida: thank you so much.

Special thanks to the employees of University of São Paulo, in particular to those of the Aeronautical Engineering Department for the help and management of working environment through this time. You are also responsible to enable research development here. Yet, thanks to the National Council for Scientific and Technological Development $(\mathrm{CNPq})$ and the Coordination for the Improvement of Higher Education Personnel (CAPES) for the financial support of this work and of the Graduate Program in Mechanical Engineering of EESC/USP. Without it, I would not be able to have the opportunity to start and finish this work.

I could not let pass the opportunity to thank all the people involved in the treatment of my sister Gabriela. During a great amount of time in the last year you were fully dedicated to reconquer life quality for her. During many moments that our hearts were broken you were there to maintain equilibrium inside our minds. And you and us triumphed towards the darkness; besides all the cruelty and harshness of the world, life can be beautiful — everything is ephemeral. 
Ultimately, to all of those that directly or indirectly are responsible for the current point of my journey: thank you! 
"Alague seu coração de esperanças, mas não deixe que se afogue nelas. Se achar que precisa voltar, volte! Se perceber que precisa seguir, siga! Se estiver tudo errado, comece novamente. Se estiver tudo certo, continue. Se sentir saudades, mate-as. Se perder um amor, não se perca! Se o achar, segure-o! Circunda-te de rosas, ama, bebe e cala. O mais é nada." 



\section{RESUMO}

\section{SOUZA, G. S. C. AVALIAÇÃO DO COMPORTAMENTO DE PLACAS LAMINADAS DE MATERIAL COMPÓSITO SOB CISALHAMENTO APÓS CARREGAMENTO DE IMPACTO. 2021. 145p. Dissertação}

(Mestrado) - Escola de Engenharia de São Carlos, Universidade de São Paulo, 2021.

Materiais compósitos laminados de matriz polimérica reforçados por fibras (MPRF) representam, na indústria aeronáutica, a maior revolução desde o advento da turbina a jato. Possuindo alta rigidez e resistência específica, estes encontram cada vez mais espaço em aplicações de alta performance e, especialmente, em aeronaves. Contudo, atualmente existem limitações em seu uso atreladas a complexidade na previsão do comportamento destes materiais sob carregamentos de impacto, o qual pode resultar em redução significativa em suas propriedades mecânicas. Além disso, devido a sua heterogeneidade e anisotropia, os mecanismos de iniciação e progressão de dano e falha não são entendidos plenamente. Desta forma, a aplicação de materiais compósitos em estruturas aeronáuticas ainda seguem filosofias de projeto conservadoras. Neste contexto, é estratégico o estudo do comportamento mecânico e do processo de falha apresentado por estes materiais. Além disso, o comportamento pós-falha, no sentido de prever sua resistência residual, é de grande importância para utilização de filosofias tolerantes ao dano ao se projetar uma dada estrutura. Assim, este trabalho apresenta uma abordagem experimental, auxiliada computacionalmente, para avaliar o comportamento de compósitos do tipo MPRF sob cisalhamento pós-impacto de baixa velocidade com reforço unidirecional tendo em vista que este é um tema pouco explorado pela literatura existente. Com base na Mecânica do Dano Contínuo, emprega-se um modelo de material da literatura afim de se investigar computacionalmente a falha e pós-falha destes materiais sob impacto e cisalhamento pós-impacto. Assim, ensaios experimentais em espécimes $\left[0^{\circ}\right]_{16}$ são conduzidos em um aparato de rail test (3 trilhos) e drop test a fim de se obter resultados de curvas tensão-deformação para os laminados com e sem dano. Baseado nestes, uma métrica de dano fenomenológica para cisalhamento pós-impacto é proposta para auxiliar na obtenção da resistência residual destes materiais. Simulações computacionais são realizadas afim de se obter via método dos elementos finitos as tendências observadas experimentalmente e para avaliar as potencialidades e limitações do modelo de material utilizado. Conclui-se ao término deste trabalho que a metodologia proposta é promissora para se obter um complementar as abordagens já consolidadas de compressão e flexão pós-impacto.

Palavras-chave: Compósitos laminados. Carregamento de impacto. Comportamento pós-impacto. Análise de elementos finitos. Cisalhamento pós-impacto. 



\section{ABSTRACT}

\section{SOUZA, G. S. C. EVALUATION OF LAMINATED COMPOSITE PLATES}

BEHAVIOR UNDER SHEAR-AFTER-IMPACT LOADING CONDITIONS: A METHODOLOGY PROPOSAL. 2021. 145p. Dissertation (Master's) - São Carlos School of Engineering, University of São Paulo, São Carlos, 2021.

Fiber-reinforced polymer (FRP) laminated composite materials represents the greatest revolution since the development of the jet turbine in aeronautical industry. Possessing high stiffness and strength to weight ratio, they encounter increasingly space in high-performance applications, particularly in aircrafts. However, nowadays there are limitations in their usage attached to the complexity of prediction of behavior that these materials presents under impact loadings, which can result in a significant reduction in their mechanical properties. Moreover, due to its heterogeneity and anisotropy, the description of initiation and propagation of damage and failure mechanisms are not fully understood yet. Thus, the application of composite materials in aeronautical structures still follow conservative design philosophies. In this context, it is strategic the study of the mechanical behavior and the failure development and evolution presented by these materials. Following this, the post-failure behavior comprehension, in the sense of prediction of its residual strength, it is of major relevance to use damage tolerant design philosophies when designing one structure. So, this work presents a experimental approach, computationally aided, to evaluate the shear-after (low-velocity) impact behavior of FRP composites with unidirectional (UD) reinforcement since this is a poorly explored theme by the existent literature. Based on Continuum Damage Mechanics (CDM), a material model present in the literature is employed to investigate computationally the failure and post-failure of these materials under impact an shear-after-impact loadings. Thus, experimental tests in $\left[0^{\circ}\right]_{16}$ specimens are conducted in a 3-rail and drop-weight tests apparatus to obtain the stress-strain curves results for laminates with and without damage. Based in these, a phenomenological damage metric for shear-after-impact is proposed to aid the obtaining of the residual strength of these materials. Computational simulations are realized aiming to obtain, using the finite element method, the tendencies experimentally observed and to evaluate the potentialities and limitations of the material model employed. It is concluded at the end of this work that the proposed methodology is promising to obtain a complementary to the already consolidated approaches of compression- and flexure-after-impact.

Keywords: Laminated composites. Impact loading. Post-impact behavior. Finite element analysis. Shear-after-impact. 



\section{LIST OF FIGURES}

Figure 1 - Boeing 787 dreamliner (a) and Airbus A380 (b) . . . . . . . . . . . 3

Figure 2 - Northrop Grumman B-2 Spirit. . . . . . . . . . . . . . . 3

Figure 3 - Stress in a cubic element. . . . . . . . . . . . . . . . 9 9

Figure 4 - Engineering and tensorial shear strains. . . . . . . . . . . . . 10

Figure 5 - Lamina numbering system for a laminate. . . . . . . . . . . . . . . 13

Figure 6 - Comparison between large (a) and small (b) mass impact. . . . . . . 16

Figure 7 - Impact Characterization Diagram. . . . . . . . . . . . . . . . 18

Figure 8 - Delaminated area orientation and morphology. . . . . . . . . . . . . 19

Figure 9 - Matrix and fiber failures. . . . . . . . . . . . . . . . . 20

Figure 10 - Shear cracks (a) and tensile crack (b) scheme. . . . . . . . . . . . . . . 21

Figure 11 - Pine tree (a) and reversed pine tree (b) patterns for thick and thin laminates, respectively. . . . . . . . . . . . . . . . . . . 21

Figure 12 - Example of damage left by LEI on a thin composite plate on both sides. 22

Figure 13 - Compression residual strength as a function of the permanent indentation after impact. . . . . . . . . . . . . . . . . . . 23

Figure 14 - Schematic of design load levels as a function of damage severity. . . . . 24

Figure 15 - Damage size and inspection type dependency. . . . . . . . . . . . . . 25

Figure 16 - Properties degradation as proposed by Hahn and Tsai. . . . . . . . . . 27

Figure 17 - Intralaminar failure modes. . . . . . . . . . . . . . . . . . 28

Figure 18 - Kink band region (left) and fiber misalignment represented by local waviness (right) at some lamina. . . . . . . . . . . . . . . . 28

Figure 19 - Fracture of a UD lamina submitted to transverse compression and in-plane shear. . . . . . . . . . . . . . . . . . . . . . . . 29

Figure 20 - Schematic representation of crack initiation and scales of analyses. . . . 30

Figure 21 - Continuum damage mechanics concept for composite materials. . . . . 32

Figure 22 - Representative volume element with damage. . . . . . . . . . . . . . . 33

Figure 23 - Hypothetical compression stress-strain experimental curve (a), secant modulus (b) and successive secant modulus (c). . . . . . . . . . . 37

Figure 24 - Ply damage threshold limits. . . . . . . . . . . . . . . . . . . . . . 39

Figure 25 - Stiffness degradation due to damage and parameters calculation procedure. 41

Figure 26 - Damage evolution due to shear (a) and transverse tension (b). . . . . . 42

Figure 27 - Specimen fixture (a) and rigid base (b) described in the ASTM D7136 standard. . . . . . . . . . . . . . . . . . . 4 44

Figure 28 - Specimen fixture of the ASTM D5628 standard. . . . . . . . . . . . . . 44

Figure 29 - Force (a) and energy (b) histories examples for several LVI energy levels. 45

Figure 30 - Energy history scheme: absorbed and elastic portions. . . . . . . . . . . 46 
Figure 31 - Displacement against force plot. . . . . . . . . . . . . . . . . . 47

Figure 32 - Absorbed energy x Impact energy. . . . . . . . . . . . . . . . . . . . . . 47

Figure 33 - Overview of impact analysis methods. . . . . . . . . . . . . . . . . . . 48

Figure 34 - Fan-shaped delamination morphology. . . . . . . . . . . . . . . 51

Figure 35 - UMAT subroutine (a) and VUMAT subroutine schemes (b). . . . . . . 55

Figure 36 - Iosipescu and V-notched rail tests. . . . . . . . . . . . . . . 57

Figure 37 - Principle of 3-rail test: setup (a); induced deformation (b) . . . . . . . 58

Figure 38 - Shear stress-strain curves of L700 laminate. . . . . . . . . . . . . . . 59

Figure 39 - Typical scenario of damage and failure under CAI. . . . . . . . . . . . 62

Figure 40 - Compression-after-impact support fixture . . . . . . . . . . . . . . 63

Figure 41 - Oven for curing (a), adopted lamination sequence with required components

(b) and vacuum bag scheme (c) . . . . . . . . . . . . . . . 66 66

Figure 42 - Expected and experimental cure cycles of the composite laminated plates. 67

Figure 43 - Example of a coupon after spraying (a) and universal testing machine (b). 69

Figure 44 - Stress-strain curves for $\left[0^{\circ}\right]_{8 T}$ coupons under tension. . . . . . . . . . . 71

Figure 45 - Stress-strain curves for $\left[90^{\circ}\right]_{8 T}$ coupons under tension. . . . . . . . . . . 72

Figure 46 - Stress-strain curves for $\left[ \pm 45^{\circ}\right]_{4 s}$ coupons under tension. . . . . . . . . . 74

Figure 47 - Linear-elastic shear threshold parameter obtaining. . . . . . . . . . . 76

Figure 48 - Damage evolution for pure cases of in-plane shear (a) and transverse tension (b). . . . . . . . . . . . . . . . . 76

Figure 49 - Percentage contribution to degradation of each stress nature of off-axis laminates as a function of the fiber orientation. . . . . . . . . . 80

Figure 50 - Monotonic and cyclic stress-strain curves of $\left[45^{\circ}\right]_{6}$ (a) and $\left[70^{\circ}\right](\mathrm{b})$ off-axis coupons. . . . . . . . . . . . . . . . . . . 81

Figure 51 - Transverse (a) and shear (b) stress-strain cyclic curves for $\left[45^{\circ}\right]_{6}$ coupons: material coordinate system. . . . . . . . . . . . . . . 81

Figure 52 - Failure modes observed in off-axis $\left[70^{\circ}\right]_{6}$ (a) and $\left[45^{\circ}\right]_{6}$ (b) coupons. . . 82

Figure 53 - Damage evolution: coupled cases of transverse tension (a) in-plane shear

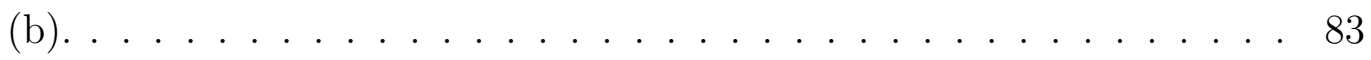

Figure 54 - Coupling coefficient $b$ as a function of the equivalent thermodynamic force. . . . . . . . . . . . . . . . . . . . 8 83

Figure 55 - Coupling coefficient $b$ as a function of the fiber orientation $\theta \ldots \ldots$. . . 84

Figure 56 - Two rail (a) and three rail (b) test standardized apparatus. . . . . . . . 85

Figure 57 - Drop-weight test complete system. . . . . . . . . . . . . . . 87

Figure 58 - Three rail apparatus for shear and SAI tests. . . . . . . . . . . . . 87

Figure 59 - Drop-tower basis with guiding pins (circled) . . . . . . . . . . . . . 88

Figure 60 - Proposed specimen for SAI testing. . . . . . . . . . . . . . . . . . 89

Figure 61 - Impact events scheme in the SAI proposed coupon. . . . . . . . . . . . 90

Figure 62 - Proposed low-energy impact evaluation strategy. . . . . . . . . . . . . . 91 
Figure 63 - Proposed methodology flowchart for SAI analysis. . . . . . . . . . . . 92

Figure 64 - Summary of SAI analysis. . . . . . . . . . . . . . . . . . 93

Figure 65 - SAI damage index: example of experimental curve defining $E_{P}$ and $E_{D}^{\min } .95$

Figure 66 - Methodology for SAI experimental analysis. . . . . . . . . . . . . . . 98

Figure 67 - Impact force (a), displacement (b) and energy (c) histories; force-displacement curves $(\mathrm{d}) \ldots \ldots \ldots \ldots 9$

Figure 68 - Shear stress-strain curves: pristine/damaged rail test and \pm 45 angle-ply coupons. . . . . . . . . . . . . . . . . . . . 101

Figure 69 - Pristine (left) and impacted (right) plates after 3-rail test. . . . . . . . 102

Figure 70 - Pristine and impacted plates after 3-rail test without usage of a drilling mask. . . . . . . . . . . . . . . . . . 102

Figure 71 - FE model of the $\left[0^{\circ}\right]_{16}$ 3-rail test specimen. . . . . . . . . . . . . . 103

Figure 72 - Comparison between shear stress-strain curves using the current work damage onset envelope and Ferreira (2014) for tensile \pm 45 angle-ply and rail test laminate. . . . . . . . . . . . . . . . . . . 105

Figure 73 - Damage variables evolution during FEA of rail tests (a) and tensile \pm 45 angle-ply (b) models. . . . . . . . . . . . . . . . . . . . 105

Figure 74 - Damage variable $d_{6}$ distribution at ultimate shear stress for rail test (a) and tensile \pm 45 angle-ply (b) computational models. . . . . . . . . . . 106

Figure 75 - Computational shear stress-strain curves: pristine/damaged rail tests FE model results. . . . . . . . . . . . . . . . . . . . . . . . . . 107

Figure 76 - DIC strain field of pristine coupon at ultimate (a) and fracture (b) shear stresses; FEM strain field of pristine coupon at fracture shear stress (c). 109

Figure 77 - DIC strain field of damaged coupon at ultimate (a) and fracture (b) shear stresses; FEM strain field of damaged coupon with $50 \%$ degradation at fracture shear stress (c). . . . . . . . . . . . . . . 110

Figure 78 - Computational FE results for tension in $\left[0^{\circ}\right]_{5}$ laminate. . . . . . . . . 128

Figure 79 - Detailed cut of FE results for tension in $\left[0^{\circ}\right]_{5}$ laminate. . . . . . . . . . 129

Figure 80 - Computational $\mathrm{FE}$ and experimental results for tension in $\left[0^{\circ}\right]_{8 T}$ laminate. 130

Figure 81 - Computational FE and experimental results for tension in $\left[ \pm 45^{\circ}\right]_{4 s}$ laminate. . . . . . . . . . . . . . . . . . . . 131

Figure 82 - Detailed cut of obtained results for tension in $\left[ \pm 45^{\circ}\right]_{4 s}$ laminate. . . . . 132

Figure 83 - Damage variables values monitoring for $\left[ \pm 45^{\circ}\right]_{4 s}$ laminate. . . . . . . . 132

Figure 84 - Damage variables monitoring: $d_{2} \ldots \ldots \ldots$. . . . . . . . . . . 134

Figure 85 - Damage variables monitoring: $d_{6}$. . . . . . . . . . . . . . . 135

Figure 86 - Force history of the drop-test for the Al 7075-T6 plate. . . . . . . . . . 138

Figure 87 - Drop-tower frame with the white paint sprayed T-beam (circled). . . . 138

Figure 88 - Displacement history of the drop-test for the Al 7075-T6 plate. . . . . . 139

Figure 89 - Force-displacement curve of the drop-test for the Al 7075-T6 plate. . . 140 
Figure 90 - Instant velocity of the drop-test for the Al 7075-T6 plate. . . . . . . . . 141

Figure 91 - Energy history of the drop-test for the Al 7075-T6 plate (complete raw data). . . . . . . . . . . . . . . . . . . 141

Figure 92 - Optimization convergence curve (a) and relative error between raw and filtered displacement data (b) . . . . . . . . . . . . . . . . . . . . . . 144

Figure 93 - Energy history of the drop-test for the Al 7075-T6 plate. . . . . . . . . 144

Figure 94 - Energy history acquirement procedure . . . . . . . . . . . . . 145 


\section{LIST OF TABLES}

Table 1 - Tensorial and contracted notations for stress and strain . . . . . . . . 9

Table 2 - Material model summary . . . . . . . . . . . . . 43

Table 3 - Summary of numerical models in literature (after Lin (LIN; WAAS, 2019)) 52

Table 4 - Obtained results for fiber/matrix volume fractions of each coupon . . . 68

Table 5 - Fiber and matrix volume fractions final results . . . . . . . . . . . 69

Table 6 - Results for the longitudinal tensile tests of $\left[0^{\circ}\right]_{8 T}$ specimens . . . . . 71

Table 7 - Results for the transverse tensile tests of $\left[90^{\circ}\right]_{8 T}$ specimens $\ldots \ldots$. . . 72

Table 8 - Results for the shear tests of $\left[ \pm 45^{\circ}\right]_{4 s}$ specimens . . . . . . . . 73

Table 9 - Carbon fiber composite properties summary . . . . . . . . . . . . 74

Table 10 - Tests and standards for material parameters identification . . . . . . . . 75

Table 11 - Compression damage modes parameters . . . . . . . . . . . . . 75

Table 12 - Damage evolution parameters for pure in-plane shear and transverse tension . . . . . . . . . . . . . . . . . 77

Table 13 - Coupling coefficient, thermodynamic force and damage variables values results for off-axis laminates (average of tested coupons) . . . . . . . . . 82

Table 14 - Drop-test parameters for the $[0]_{16}$ laminates. . . . . . . . . . . . . . . 99

Table 15 - CFRP drop-tests summary for $[0]_{16}$ laminates. . . . . . . . . . . . . 100

Table 16 - Results summary for simple (rail) and pure (angle-ply) shear comparisons: current work and Ferreira's (2014) envelopes . . . . . . . . . . . . 107

Table 17 - Shear-after-impact damage index FEM results . . . . . . . . . . . . 108

Table 18 - Tita's material properties and strength values . . . . . . . . . . . . 127

Table 19 - Tensile results for $\left[0^{\circ}\right]_{5}$ coupon comparison . . . . . . . . . . . . . 129

Table 20 - Tensile results for $\left[0^{\circ}\right]_{8 T}$ coupon comparison . . . . . . . . . . . . 130

Table 21 - Drop-test parameters for Al 7075-T6 . . . . . . . . . . . . . . . 137

Table 22 - Optimization parameters . . . . . . . . . . . . . . . 143

Table 23 - Aluminum plate drop-test summary of results . . . . . . . . . . . . 145 



\begin{tabular}{|c|c|}
\hline $1 \mathrm{D}$ & One-dimensional \\
\hline $2 \mathrm{D}$ & Two-dimensional \\
\hline $3 \mathrm{D}$ & Three-dimensional \\
\hline ACEE & Aircraft Energy Efficiency \\
\hline ASTM & American Society for Testing and Materials \\
\hline BVID & Barely Visible Impact Damage \\
\hline $\mathrm{BD}$ & Bidirectional \\
\hline $\mathrm{BC}$ & Boundary Condition \\
\hline CFRP & Carbon Fiber-Reinforced Polymer \\
\hline CLT & Classical Laminate Theory \\
\hline CZM & Cohesive Zone Method \\
\hline CAI & Compression-After-Impact \\
\hline CDM & Continuum Damage Mechanics \\
\hline DI & Damage Index \\
\hline DTL & Damage Threshold Load \\
\hline DOF & Degree Of Freedom \\
\hline $\mathrm{DIC}$ & Digital Image Correlation \\
\hline FS & Factor of Safety \\
\hline FI & Failure Index \\
\hline FFT & Fast Fourier Transform \\
\hline FRP & Fiber-Reinforced Polymer \\
\hline $\mathrm{FE}$ & Finite Element \\
\hline FEA & Finite Element Analysis \\
\hline FEM & Finite Element Method \\
\hline
\end{tabular}




\begin{tabular}{|c|c|}
\hline FPF & First Ply Failure \\
\hline FAI & Flexure-After-Impact \\
\hline FORTRAN & IBM Mathematical Formula Translation System \\
\hline GA & Genetic Algorithm \\
\hline GFRP & Glass Fiber-Reinforced Polymer \\
\hline GEA & Grupo de Estruturas Aeronáuticas \\
\hline HIV & High-Velocity Impact \\
\hline LaRC03 & Langley Research Center 03 Failure Criterion \\
\hline LDS & Laser Distance Sensor \\
\hline $\mathrm{LPF}$ & Last Ply Failure \\
\hline $\mathrm{L}$ & Longitudinal \\
\hline LEI & Low-Energy Impact \\
\hline LL & Limit Load \\
\hline LVI & Low-Velocity Impact \\
\hline MATLAB & Matrix Laboratory \\
\hline MOER & Mode One Energy Ratio \\
\hline MTS & MTS Systems Corporation \\
\hline NASA & National Aeronautics and Space Administration \\
\hline RVE & Representative Volume Element \\
\hline RFI & Resin Film Infusion \\
\hline RTM & Resin Transfer Molding \\
\hline S-G & Savitsky-Golay \\
\hline SAI & Shear-After-Impact \\
\hline SS & Shearography Speckle \\
\hline SHBT & Split-Hopkinson Bar Testing \\
\hline SHM & Structural Health Monitoring \\
\hline
\end{tabular}


Transverse

UD

Unidirectional

LL Ultimate Load

UMAT User Material Subroutine

VUMAT Vectorized User Material Subroutine

VBM Vibration Based Method

VCCT Virtual Crack Closure Technique

WWFE World-Wide Failure Exercise 



\section{LIST OF SYMBOLS}

\begin{tabular}{|c|c|}
\hline$E_{a}$ & Absorbed Energy \\
\hline $\mathrm{D}$ & Bending-Torsion Stiffness Matrix \\
\hline $\mathrm{w}_{e}$ & Complementary Energy Density \\
\hline$S_{i j}$ & Compliance Matrix \\
\hline$\omega$ & Contact Frequency \\
\hline$K_{\alpha}$ & Contact Stiffness \\
\hline$b$ & Coupling Parameter \\
\hline B & Coupling Stiffness Matrix \\
\hline$\kappa$ & Curvature \\
\hline $\mathcal{D}$ & Damage Dissipation \\
\hline DI & Damage Index \\
\hline$D_{i j k l}$ & Damage Tensor \\
\hline$d_{i}$ & Damage Variable \\
\hline$A$ & Damaged Zone \\
\hline$E_{i i}^{\mathrm{df}}$ & Degraded Young's Moduli in $i$-direction \\
\hline$\rho$ & Density \\
\hline$u$ & Displacement in the $\mathrm{x}$-direction \\
\hline$v$ & Displacement in the $\mathrm{y}$-direction \\
\hline$w$ & Displacement in the z-direction \\
\hline$\hat{\sigma}_{i j}$ & Effective Stress Tensor \\
\hline$E_{e}$ & Elastic Energy \\
\hline$\gamma_{i j}$ & Engineering Shear Strain \\
\hline$F_{\exp }$ & Experimental Force \\
\hline
\end{tabular}

A Extensional Stiffness Matrix 


\begin{tabular}{|c|c|}
\hline$V_{f}$ & Fiber Volume Fraction \\
\hline$F$ & Force \\
\hline$\underline{\mathrm{N}}$ & Force Resultants \\
\hline$g$ & Gravitational Acceleration \\
\hline$h$ & Height \\
\hline$E_{i}$ & Impact Energy \\
\hline$M_{i}$ & Impactor Mass \\
\hline$R_{i}$ & Impactor Radius \\
\hline$I_{1}$ & Inertial Parameter \\
\hline$v_{0}$ & Initial Velocity \\
\hline$S^{L}$ & In-Plane Shear Strength \\
\hline$A_{0}$ & Interaction Failure Zone \\
\hline $\mathcal{K E}$ & Kinetic Energy \\
\hline$\delta_{i j}$ & Kronecker's Delta \\
\hline$X_{C}$ & Longitudinal Compressive Strength \\
\hline$X_{T}$ & Longitudinal Tensile Strength \\
\hline$E_{11}$ & Longitudinal Young's Modulus \\
\hline$\zeta_{w}$ & Loss Factor \\
\hline$\nu_{12}$ & Major Poisson's Ratio \\
\hline$m$ & Mass \\
\hline$\underline{\kappa}$ & Mid-Plane Curvatures \\
\hline$\underline{\varepsilon}^{0}$ & Mid-Plane Engineering Strains \\
\hline$E_{D}^{\min }$ & Minimum Allowable Toughness \\
\hline$\nu_{21}$ & Minor Poisson's Ratio \\
\hline$\underline{\mathrm{M}}$ & Moment Resultants \\
\hline $\bar{F}$ & Normalized Force \\
\hline
\end{tabular}




\begin{tabular}{|c|c|}
\hline $\bar{t}$ & Normalized Time \\
\hline$E_{33}$ & Out-of-plane Young's Modulus \\
\hline$K_{b s}$ & Plate Bending-Shear Stiffness \\
\hline$D^{*}$ & Plate Effective Bending Stiffness \\
\hline$M_{p}$ & Plate Mass \\
\hline$\nu_{i j}$ & Poisson's Ratio in the $i-j$ planes \\
\hline$Q_{i j}$ & Reduced Stiffness Matrix \\
\hline$\lambda$ & Relative Stiffness \\
\hline$G_{i j}$ & Shear Moduli in the $i$ - $j$ planes \\
\hline$\tau$ & Shear Stress \\
\hline$C_{i j}$ & Stiffness Matrix \\
\hline$\varepsilon$ & Strain \\
\hline$\sigma$ & Stress \\
\hline$\hat{Y}$ & Thermodynamic Forces for Combined Stress State \\
\hline$Y_{j}$ & Thermodynamic Variable \\
\hline$E_{D}$ & Toughness of the Damaged Coupon \\
\hline$E_{P}$ & Toughness of the Pristine Coupon \\
\hline$t$ & Time \\
\hline $\bar{Q}_{i j}$ & Transformed Reduced Stiffness Matrix \\
\hline$Y_{C}$ & Transverse Compressive Strength \\
\hline$Y_{T}$ & Transverse Tensile Strength \\
\hline$E_{22}$ & Transverse Young's Modulus \\
\hline$v$ & Velocity \\
\hline$V$ & Voltage \\
\hline$W$ & Work \\
\hline$E_{i i}$ & Young's Moduli in $i$-direction \\
\hline
\end{tabular}





\section{CONTENTS}

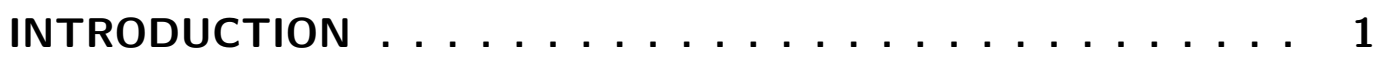

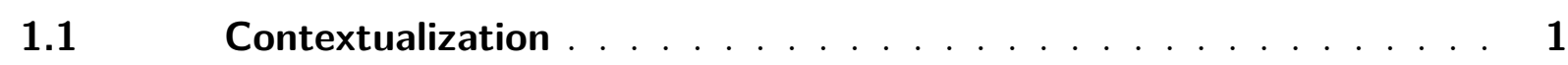

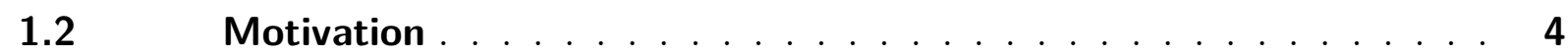

1.3 Dissertation Goals . . . . . . . . . . . . . . . . . . 5

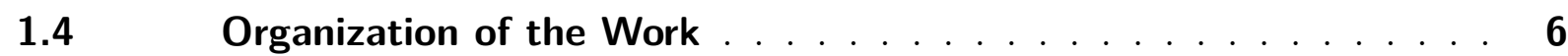

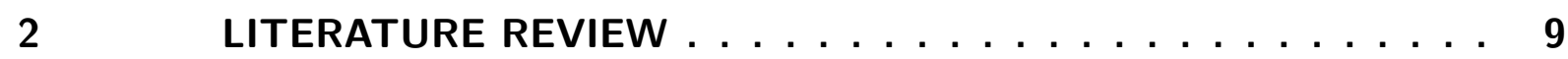

$2.1 \quad$ Stress-Strain Relations for Anisotropic Elasticity . . . . . . . . . 9

$2.2 \quad$ Impact on Composite Materials . . . . . . . . . . . . 15

2.2.1 Impact Induced Damage . . . . . . . . . . . . . . . . . . . 19

2.2.2 Barely-Visible Impact Damage . . . . . . . . . . . . . . . . . 22

2.3 Damage, Degradation and Failure of Composite Materials . . . . . 25

2.3.1 Continuum Damage Mechanics . . . . . . . . . . . . . . . . . . . . . . 29

2.3.2 Developed Material Models . . . . . . . . . . . . . . . . . . 36

$2.4 \quad$ Impact Analyses for Composite Materials . . . . . . . . . . . . 43

2.4.1 Experimental Approach . . . . . . . . . . . . . . . . . . . 43

2.4.2 Computational Approach . . . . . . . . . . . . . . . 48

$2.5 \quad$ Shear Testing of Composite Materials . . . . . . . . . . . 56

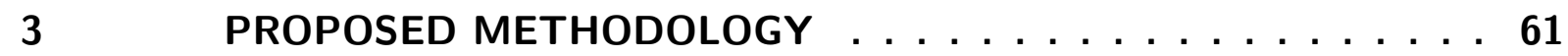

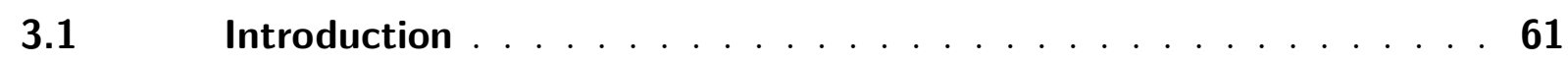

3.2 Post-Impact Methodologies for Composite Materials . . . . . . . . 61

3.2.1 Compression-After-Impact . . . . . . . . . . . . . . . 61

3.2.2 Flexure-After-Impact . . . . . . . . . . . . . . 63

$3.3 \quad$ Materials and Methods . . . . . . . . . . . . . . . . 64

3.3.1 Fiber and Matrix Contents . . . . . . . . . . . . . . . 68

3.3.2 Monotonic Quasi-Static Material Characterization Tests . . . . . . . . . . . 69

3.3.3 Summary of Monotonic Quasi-Static Tests Results . . . . . . . . . . . . 74

3.3.4 Cyclic Quasi-Static Tests and Material Model Parameters Identification . . 75

3.4 Methodology for Shear-After-Impact . . . . . . . . . . . 84

$3.4 .1 \quad$ Rail Test . . . . . . . . . . . . . . . . . . . . 84

3.4.2 Drop-Weight Specimen Adaptation . . . . . . . . . . . . . . . . 86

3.4.3 Proposed Methodology for SAI . . . . . . . . . . . . . . . . . . . . . 91

3.4.4 SAl Damage Index . . . . . . . . . . . . . . . . . . . . . . . . 94 
$4.1 \quad$ Experimental Methodology . . . . . . . . . . . . . . . . . 97

$4.2 \quad$ Results and Discussion . . . . . . . . . . . . . . . . . . . 99

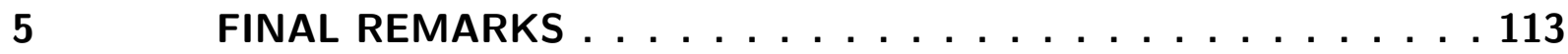

$5.1 \quad$ Conclusions . . . . . . . . . . . . . . . . . . 113

$5.2 \quad$ Future Works . . . . . . . . . . . . . . . . . . . 114

BIBLIOGRAPHY .................. 117

APPENDIX

APPENDIX A-COMPUTATIONAL INVESTIGATIONS OF QUASI-STATIC TESTS . . . . . . . . . . . . 127

APPENDIX B - DROP-TOWER CALIBRATION . . . . . . . . 137 


\section{INTRODUCTION}

\subsection{Contextualization}

Due to the necessity of designing extremely light structures, especially for the aeronautical industry, engineers search increasingly for materials that own high specific strength and stiffness. Among these, composite materials play an important role because aircraft structures are very weight-sensitive. In this sense, composites are ideal for some applications in which they are cost-effective. There are many types of composite materials and, by definition, they are composed of two or more distinct constituents possessing a matrix and a reinforcement phase (HERAKOVICH, 1998). This combination is at the macroscopic level and gives origin to a new material with unique desirable properties, i.e. a composite usually is achieved by combining two different materials that contributes isolatedly for the final properties of the former one; thus, a composite is heterogeneous. Some of these characteristics that can be improved are: strength; stiffness; acoustic/thermal insulation; weight; fatigue life; corrosion/wear resistance; among others (JONES, 1999). In this text, attention is given to the fiber-reinforced polymer (FRP) type of composite that is made of a polymer matrix reinforced with continuous fibers (usually glass, carbon or aramid). Hereafter, the word composite material (or simply composite) is associated with this type of material.

Besides all the advantages of composites, there are some challenges involving its usage. As an example, for metals with ductile behavior it is widely accepted that until the yield limit one can use von Mises failure criterion to evaluate the safety margin of one structure subjected to some kind of loading. That is not true for composites. The failure mechanisms are much more complex than that which play role in metals. There are several damage and failure modes that can be considered to fully predict the mechanical response of these materials. However, these modes are not fully understood yet (HINTON; KADDOUR; SODEN, 2004; KADDOUR; HINTON, 2013). Consequently, this makes designing with composite materials a much more difficult task than with metals.

Laminated composites are ensembles made of several layers called laminae that are stacked together to build a plate-like structures. There are many ways to manufacture this type of material, namely: hand-layup, resin transfer molding (RTM), resin film infusion (RFI), automated fiber placement or filament winding, to name a few (HERAKOVICH, 1998). This type of structure usually does not possess significant transverse strength being more susceptible to impact damage than a metallic plate. Thinking of an aircraft, damage caused by this kind of loading can arise from numerous ways such as tool dropping during the manufacturing or maintenance process, flying debris over take-off and landing procedures, bird strike, collision with another vehicle, among others (ABRATE, 1998). The 
first example usually belongs to the category of low-velocity impacts (LVI). It is important to note that this classification depends of several features besides its velocity such as geometry of the impactor, constitution of the target and others. Thus, it is preferable and more accurate classifying it as low-energy impact (LEI) although it still lacks important information about the phenomena. In this context, one needs to strictly specify the conditions in which impact takes place. For example, through this text the phenomenon is investigated in controlled conditions of a drop-weight machine where variables such as distance, velocity, mass and geometry of the impactor are well defined.

Low-energy impacts causes minimal superficial damage in composite laminates. However, it can cause severe internal damage or even failure of the structure that cannot be detected by naked eye which highly influences residual strength of the plate. Generally, this type of damage is classified as barely visible impact damage (BVID) and because of its characteristics can remain undetectable (THORSSON; WAAS; RASSAIAN, 2018a). Depending on the orientations of lamina in the laminate, impact can give rise to intralaminar damage and interlaminar failure. Specially, the last one is of much concern to the performance of the structure and usually is characterized by delamination. By definition, a delamination is the physical separation between neighbor lamina which drastically reduces the strength and stiffness of the laminate. It occurs when the stress state between these lamina overcomes the interlaminar strength (TITA, 2003). Therefore, the structural engineer needs to expend design time in well defining the material behavior under such conditions.

Composite materials are currently used in numerous types of industry applications. For example, there are the automobile, aerospace, sports, naval and medical industries. Ideal usage of composites are those where high stiffness-weight and strength-weight ratios are needed. Therefore, at aerospace industry this is justified since it is a very competitive market. In an aircraft, composite structures usage affects directly the performance by increasing its payload and range. Back in the 70s, where the application of composite material in the aeronautical industry started, the Aircraft Energy Efficiency project (ACEE), developed by NASA (National Aeronautics and Space Administration), used composites to substitute metals in some primary and mostly in secondary aeroplane structures. As a result, in some cases it was obtained reduction in $30 \%$ of the structure self-weight when compared with its metallic counterpart (NIU, 1992). These results represented a design paradigm change in structural engineering being considered the great technical revolution since the jet turbine (JONES, 1999). Nowadays, composites are used in several primary aircraft structures such as wing ribs, fuselage and wing panels, among others. As main examples of its usage in aircraft civil industry, there are the Boeing 787 dreamliner (Fig. 1a) and the Airbus A380 (Fig. 1b) where the first one has its structures composed by almost $50 \%$ of composite materials. 
Figure 1: Boeing 787 dreamliner (a) and Airbus A380 (b).

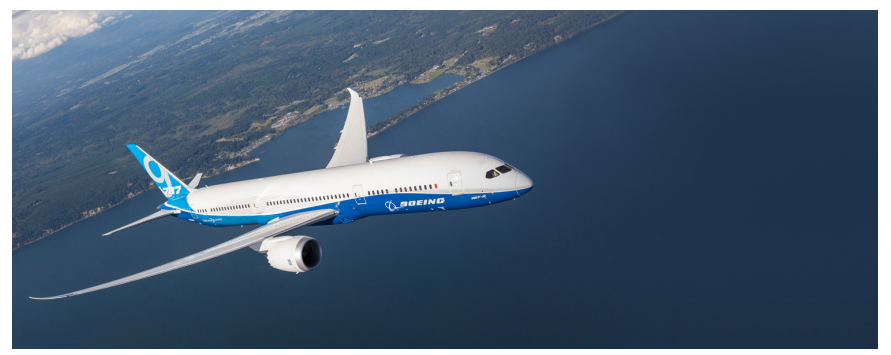

(a)

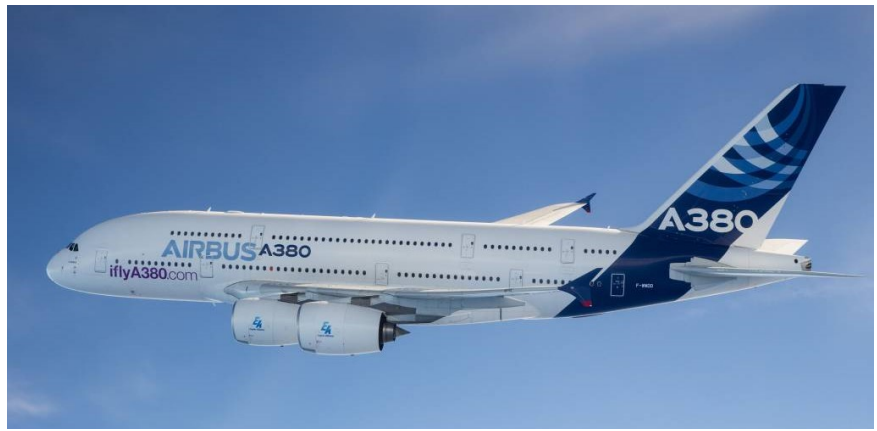

(b)

Sources: The Boeing Company (a); Airbus SE (b).

In military aircraft industry the Northrop Grumman B-2 Spirit, Boeing F-18 and Lockheed Martin F-22 can be cited as good examples of composite materials usage. Specially, it is highlighted that the B-2 Spirit (Fig. 2) possesses more than $50 \%$ of its structures composed by composites.

Figure 2: Northrop Grumman B-2 Spirit.

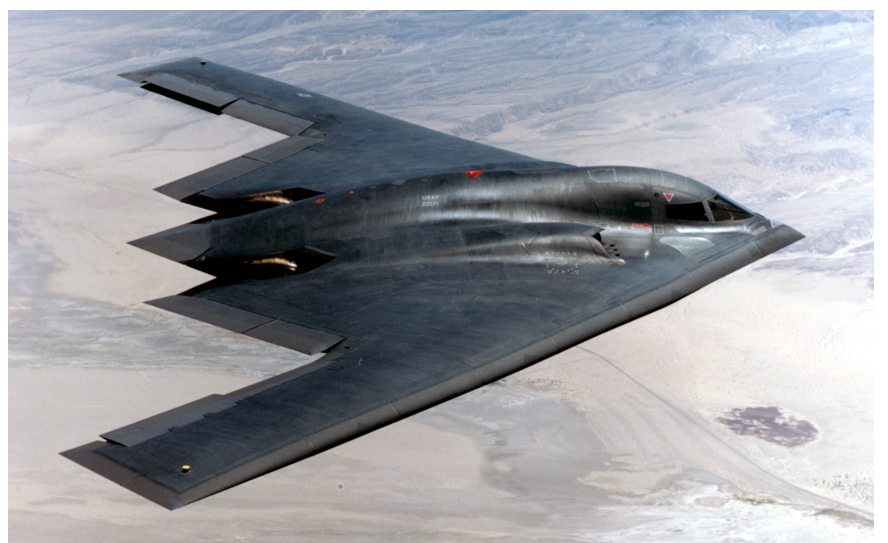

Source: Wikipedia.

Numerous successful cases can be listed where the application of composite materials was made at different industry sectors. Therefore, it is evident that composites are part 
not only of the present but also of the future of industry and academia.

\subsection{Motivation}

Airworthiness in civil aircraft industry is of much concern since passengers must have a good experience during flight. Thus, composite materials still have limited applications in this sector due to difficulties during the certification process. Challenges in the prediction of composite failure modes and in its life during operational service (TRAVESA, 2006) implies in structures designed with an infinite life philosophy. Thus, these structures are designed to not experience fatigue when considering its loading envelopes. This translates in a conservative design as the weight-saving concept cannot be applied in its full capacity. Of course, aeronautic certification authorities must guarantee that human life is preserved and worry about safety.

In this context, impact loadings that occur during the aircraft operational time must also be taken into account when designing a composite structure. Due to the complexity of the problem, many studies with the focus of overcoming these limitations were conducted in the past decades and, at some level, obtained successful results. These works follows analytical, computational and experimental approaches. Of course, an analytical strategy is the less time consuming and the cheapest one. However, certification authorities commonly requires one or both of the other analysis types to be made in conjunction with it. Regarding to experimental approaches, it is the most time consuming and expensive but it is also the one that produces the higher-fidelity results since no approximations of material behavior are made, for example. Also, to be representative of reality, an experimental analysis has to be repeated by a significant amount of times which can reveal being an almost impossible task in some cases. Thus, pure computational or hybrid computational-experimental approaches are currently used to model the composites material behavior when under impact loadings. The first one sometimes lacks exactly of experimental data to support its findings and, in this sense, hybrid strategies started to be a trend between analysts. In these approaches, a trade-off between experimental and computational results data is done. For example, a computational analysis can be conducted to guarantee that the impact is of the LVI family while quasi-static (monotonic or cyclic) tensile tests can be done for the material model parameters identification. Nowadays, this is the most powerful type of approach to, essentially, any kind of engineering problem with this inherent level of complexity.

According to Bogenfeld et al. (BOGENFELD; KREIKEMEIER; WILLE, 2018), the current challenge is the application of impact analysis in the structural level. It is worth mentioning that the evaluation of scale effects for this kind of problem on composite materials is an interesting and difficult subject of study that escapes from the scope of this work. On the other hand, sufficiently accurate models are available nowadays for predicting 
with good accuracy the response and failure behavior of composite laminated plates at the coupon scale of analysis. However, the after impact behavior of such specimens is currently being studied and still needs full comprehension. Several studies available in the literature appoints for quite reduction of compression strength of these structures on events of low-velocity impact (THORSSON; WAAS; RASSAIAN, 2018a). Furthermore, there are certain studies accounting for the flexural strength after impact (MEDEIROS, 2016) but only a few considering the shear after impact (SAI) resistance of such laminates (FENG et al., 2017b) under BVID conditions. In this context, it is important to study the SAI behavior of these structures since that once in operation, laminated composite structures are subjected to combined stress states which in most cases includes shear.

Therefore, the development of material models capable of reducing the number of experimental tests and promoting economy on the development of structural design is of great significance to the progression of composite materials application in industry.

\subsection{Dissertation Goals}

As aforementioned, the study of laminated composite plates with unidirectional reinforcement under quasi-static and face-on impact loadings is of great relevance for academia and industry. Besides several studies were conducted with the objective of evaluating the compression after impact (CAI) and flexure after impact (FAI) behavior of FRP composites, only a few aiming the SAI behavior were conducted throughout the last years. Thus, this work arises due to the lack of computational and experimental studies in the subject aiming the fulfillment of the gap that exists in the area.

In this sense, the main goal of the present work consists on proposing a methodology to evaluate the residual strength of composite laminates under shear-after-impact conditions. Therefore, some specific goals of this work are listed:

- Obtain material model parameters: experimental characterization of the in-house hand-layup manufactured specimens;

- Have a computational code: computational analysis via subroutines written in FORTRAN language and linked to ABAQUS finite element package;

- Compare computational results with experimental data: evaluate the potentialities and limitations of the material model;

- Develop a methodology considering SAI: provided with experimental and finite element method results, propose a methodology for shear-after-impact behavior assessment. 
To fulfill these objectives, reproduction of the computational results done by Ferreira (FERREIRA, 2014) are firstly made. For quasi-static simulations an UMAT (User Material Subroutine - ABAQUS Standard) is used. Once the preliminary analyses and material characterization are done, drop-weight tests are conducted with the manufactured coupons. An usual stacking sequence of $\left[0^{\circ}\right]_{16}$ of carbon fiber-reinforced polymer (CFRP) is used. At the end of this work, novel experimental guidelines for SAI testing aided by computational investigations based on the aforementioned methods and standards are proposed.

\subsection{Organization of the Work}

The present work is organized in five chapters, each of them with its themes, scopes and sections. Chapter 1 presents an overview on composite materials covering the subtopics of damage and failure caused by impact and quasi-static loadings in a brief contextualization of the theme. The relevance of the subject is highlighted giving attention to currently existing challenges in the prediction of the material behavior post-impact conditions. Motivations, goals and scope of this dissertation are presented. Chapter 2 is destined to a literature review on the themes addressed across this text. In this, the stress-strain relations for anisotropic elasticity are introduced and a more general discussion about impact mechanics on composite materials is done. The damage and failure mechanisms in these materials that occur under impact events are exposed and special attention is given to the barely-visible impact damage (BVID) type. Failure criteria, degradation laws and failure modes for composite materials are discussed. Continuum damage mechanics fundamentals and its application for composite materials failure modeling are presented and the mathematical formulation of the employed material model is introduced. A review in experimental and computational analyses of impact is presented where, for the second approach, the Finite Element Method (FEM) is focused with the implementation of user material subroutines (mainly UMAT) in ABAQUS. Lastly, an overview on the most used shear test methods for composite materials is done. In Chapter 3 post-impact CAI and FAI methodologies are discussed to provide a solid basis for the SAI methodology proposed. There is a section dedicated to materials and methods where manufacturing strategies, material characterization by means of monotonic tests and needed parameters identification via cyclic tests is performed. A new specimen is proposed for the shear-after-impact experimental analysis and the adopted damage metric is discussed. Provided with literature review and the proposed methodology, Chapter 4 deals with the experimental approach for SAI in composite materials aided by computational analyses. There is a brief introduction, followed by the experimental methodology employed and then, the obtained results are presented and discussed. Also, finite elements analyses are performed to evaluate the fidelity of the material model employed in obtaining the results needed for the SAI study. Finally, Chapter 5 compiles the results obtained, dealing with the conclusions of the present work and discuss about future works proposals. To fulfill these objectives some preliminary 
computational and experimental testing results needs to be realized and obtained. Thus, in Appendices A and B these preliminary remarks are shown and discussed. Those are used as the basis for the approach proposed through this text. 



\section{LITERATURE REVIEW}

\subsection{Stress-Strain Relations for Anisotropic Elasticity}

Hooke's Law generalized for three-dimensional (3D) anisotropy relating stress and strain in contracted notation is,

$$
\sigma_{i}=C_{i j} \varepsilon_{j}
$$

where $\sigma_{i}(i=1, \ldots, 6)$ are the stress components of a cubic 3D element in directions 1,2 and $3, C_{i j}$ is the stiffness matrix and $\varepsilon_{j}$ are the strain components as shown in Figure 3.

Figure 3: Stress in a cubic element.

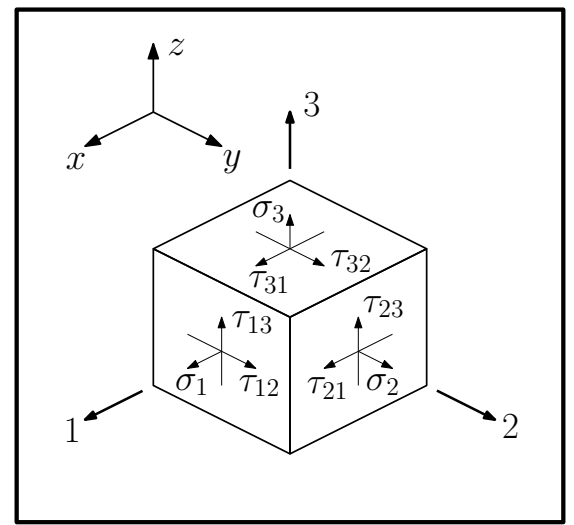

Source: by the author.

The contracted notation for 3D stress and strain is defined in Table 1 with relation to the usual tensorial notation for symmetric stress and strain tensors cases. In this, $\gamma_{i j}$ are the engineering shear strains and $\varepsilon_{i j}$ are the tensorial shear strains.

Table 1: Tensorial and contracted notations for stress and strain

\begin{tabular}{cccc}
\hline \multicolumn{2}{c}{ Stress } & \multicolumn{2}{c}{ Strain } \\
\hline Tensorial Notation & Contracted Notation & Tensorial Notation & Contracted Notation \\
\hline$\sigma_{11}$ & $\sigma_{1}$ & $\varepsilon_{11}$ & $\varepsilon_{1}$ \\
$\sigma_{22}$ & $\sigma_{2}$ & $\varepsilon_{22}$ & $\varepsilon_{2}$ \\
$\sigma_{33}$ & $\sigma_{3}$ & $\varepsilon_{33}$ & $\varepsilon_{3}$ \\
$\tau_{23}=\sigma_{32}$ & $\sigma_{4}$ & $\gamma_{23}=2 \varepsilon_{23}$ & $\varepsilon_{4}$ \\
$\tau_{31}=\sigma_{31}$ & $\sigma_{5}$ & $\gamma_{31}=2 \varepsilon_{31}$ & $\varepsilon_{5}$ \\
$\tau_{12}=\sigma_{12}$ & $\sigma_{6}$ & $\gamma_{12}=2 \varepsilon_{12}$ & $\varepsilon_{6}$ \\
\hline
\end{tabular}


Thus, for small deformations, strains are defined as (JONES, 1999):

$$
\begin{aligned}
\varepsilon_{1} & =\frac{\partial u}{\partial x}, \\
\varepsilon_{2} & =\frac{\partial v}{\partial y}, \\
\varepsilon_{3} & =\frac{\partial w}{\partial z},
\end{aligned}
$$

and,

$$
\begin{aligned}
\gamma_{23} & =\frac{\partial v}{\partial z}+\frac{\partial w}{\partial y} \\
\gamma_{31} & =\frac{\partial w}{\partial x}+\frac{\partial u}{\partial z} \\
\gamma_{12} & =\frac{\partial u}{\partial y}+\frac{\partial v}{\partial x}
\end{aligned}
$$

where $u, v$ and $w$ are displacements in the $x, y$ and $z$ directions, respectively. Also, Eqs. (2.2) and (2.3) are only valid for small strains and displacements.

Figure 4 highlights the difference between engineering and tensorial shear strains. Notice that the $\gamma_{i j}$ strain is the total angle that the element experiences when submitted to a simple shear state. On the other hand, the $\varepsilon_{i j}$ strain represents half of this " $\gamma_{i j}$ " angle that the same element experiences when submitted to a pure shear state. Physically, engineering shear strain implies in a rotation of the element while tensorial shear strain is followed by a stretching of it.

Figure 4: Engineering and tensorial shear strains.

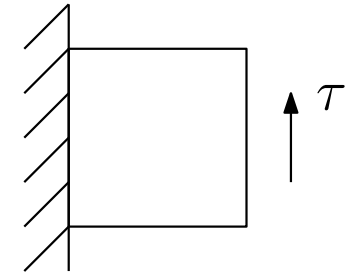

Simple shear

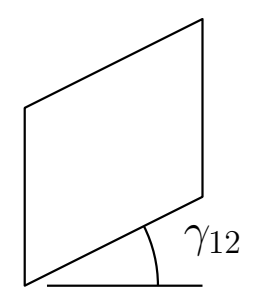

Engineering shear strain

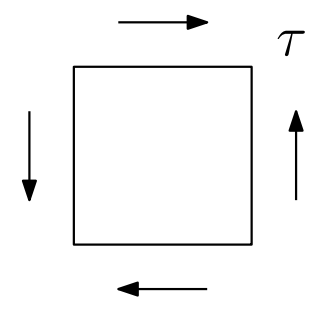

Pure shear

$$
\gamma_{12}=2 \varepsilon_{12}
$$

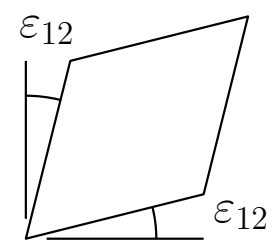

Tensorial shear strain

Source: adapted from (JONES, 1999) by the author.

The stiffness (or constitutive) matrix in Eq. (2.1) possess 36 constants. This number can be reduced when considering important strain energy aspects. Elastic materials, for 
which a elastic potential exists and is equal to the strain energy density (LEKHNITSKII, 1965), has an increment of specific work given by (JONES, 1999),

$$
d W=\sigma_{i} d \varepsilon_{i}
$$

when stresses $\sigma_{i}$ acts by means of the incremental strains $d \varepsilon_{i}$. Making use of Eq. (2.1), it follows:

$$
d W=C_{i j} \varepsilon_{j} d \varepsilon_{j}
$$

Integrating the last with respect to the strains, specific work is given by,

$$
W=\frac{1}{2} C_{i j} \varepsilon_{i} \varepsilon_{j}
$$

On the other hand, Hooke's Law can be obtained from Eq. (2.6):

$$
\frac{\partial W}{\partial \varepsilon_{i}}=C_{i j} \varepsilon_{j}
$$

and, taking the second derivative,

$$
\frac{\partial^{2} W}{\partial \varepsilon_{i} \partial \varepsilon_{j}}=C_{i j}
$$

Analogously, it follows that,

$$
\frac{\partial^{2} W}{\partial \varepsilon_{j} \partial \varepsilon_{i}}=C_{j i}
$$

Since the derivative of $W$ is immaterial ${ }^{1}$ (JONES, 1999), it is trivial that:

$$
C_{i j}=C_{j i}
$$

Hence, the stiffness matrix is symmetric and possess 21 different constants but only 18 of them are independent (JONES, 1999). Another way to prove Eq. (2.10) is to demonstrate that the stress and strain tensors are both symmetric and, consequently, the stiffness tensor is symmetric as well. Hypothetically, it is considered a continuous media that follows the generalized Hooke's law. Moreover, only small strains are taken into account. Thus, the strain components are linear functions of the stress components (LEKHNITSKII, 1965). So, it follows immediately that Eq. (2.10) is valid.

Similarly, $W$ can be expressed in terms of compliance and stress. Thus, differentiating twice with respect to stresses, it can be proven that $S_{i j}=S_{j i}$. That is, the compliance

1 The order of $i_{1}, \ldots, i_{k}$ is immaterial on the differentiation of a function $f$ with respect to the variables $x_{i_{1}}, \ldots, x_{i_{k}}$ if the same possesses continuous partial derivatives of all orders. Functions with this property are called $C^{\infty}$ functions (SPIVAK, 1971). This result is known as Clairaut-Schwarz Theorem (HUBBARD; HUBBARD, 2015). 
matrix $S_{i j}$ is also symmetric. Finally, the stress-strain relations for linear-elastic anisotropic materials is given by (GIBSON, 2012):

$$
\left\{\begin{array}{c}
\sigma_{1} \\
\sigma_{2} \\
\sigma_{3} \\
\tau_{23} \\
\tau_{31} \\
\tau_{12}
\end{array}\right\}=\left[\begin{array}{cccccc}
C_{11} & C_{12} & C_{13} & C_{14} & C_{15} & C_{16} \\
& C_{22} & C_{23} & C_{24} & C_{25} & C_{26} \\
& & C_{33} & C_{34} & C_{35} & C_{36} \\
& & & C_{44} & C_{45} & C_{46} \\
& & & & C_{55} & C_{56} \\
& & & & & C_{66}
\end{array}\right]\left\{\begin{array}{c}
\varepsilon_{1} \\
\varepsilon_{2} \\
\varepsilon_{3} \\
\gamma_{23} \\
\gamma_{31} \\
\gamma_{12}
\end{array}\right\} .
$$

where there are no symmetry planes in the material. For the case where exists two planes of material symmetry, it follows that symmetry will also exist for a third mutually orthogonal plane with respect to both of them. For a coordinate system aligned with the materials directions (1, 2 and 3), Hooke's law is given as (JONES, 1999),

$$
\left\{\begin{array}{c}
\sigma_{1} \\
\sigma_{2} \\
\sigma_{3} \\
\tau_{23} \\
\tau_{31} \\
\tau_{12}
\end{array}\right\}=\left[\begin{array}{cccccc}
C_{11} & C_{12} & C_{13} & 0 & 0 & 0 \\
C_{12} & C_{22} & C_{23} & 0 & 0 & 0 \\
C_{13} & C_{23} & C_{33} & 0 & 0 & 0 \\
0 & 0 & 0 & C_{44} & 0 & 0 \\
0 & 0 & 0 & 0 & C_{55} & 0 \\
0 & 0 & 0 & 0 & 0 & C_{66}
\end{array}\right]\left\{\begin{array}{c}
\varepsilon_{1} \\
\varepsilon_{2} \\
\varepsilon_{3} \\
\gamma_{23} \\
\gamma_{31} \\
\gamma_{12}
\end{array}\right\},
$$

and these materials are said orthotropic. Notice that materials with this property possesses only 9 independent constants in the stiffness matrix.

It is more interesting to write Eq. (2.12) by means of the compliance matrix since its terms can be directly related to elastic material properties that are measured in laboratory experiments (HERAKOVICH, 1998). It follows that,

$$
\left\{\begin{array}{c}
\varepsilon_{1} \\
\varepsilon_{2} \\
\varepsilon_{3} \\
\gamma_{23} \\
\gamma_{31} \\
\gamma_{12}
\end{array}\right\}=\left[\begin{array}{cccccc}
S_{11} & S_{12} & S_{13} & 0 & 0 & 0 \\
S_{21} & S_{22} & S_{23} & 0 & 0 & 0 \\
S_{31} & S_{32} & S_{33} & 0 & 0 & 0 \\
0 & 0 & 0 & S_{44} & 0 & 0 \\
0 & 0 & 0 & 0 & S_{55} & 0 \\
0 & 0 & 0 & 0 & 0 & S_{66}
\end{array}\right]\left\{\begin{array}{c}
\sigma_{1} \\
\sigma_{2} \\
\sigma_{3} \\
\tau_{23} \\
\tau_{31} \\
\tau_{12}
\end{array}\right\},
$$

where $S_{i j}=C_{i j}^{-1}$ and, in terms of elastic material properties,

$$
\begin{aligned}
& S_{11}=\frac{1}{E_{11}}, \quad S_{12}=-\frac{\nu_{21}}{E_{22}}, \quad S_{13}=-\frac{\nu_{31}}{E_{33}}, \\
& S_{21}=-\frac{\nu_{12}}{E_{11}}, \quad S_{22}=\frac{1}{E_{22}}, \quad S_{23}=-\frac{\nu_{32}}{E_{33}}, \\
& S_{31}=-\frac{\nu_{13}}{E_{11}}, \quad S_{32}=-\frac{\nu_{23}}{E_{22}}, \quad S_{33}=\frac{1}{E_{33}}, \\
& S_{44}=\frac{1}{G_{23}}, \quad S_{55}=\frac{1}{G_{13}}, \quad S_{66}=\frac{1}{G_{12}},
\end{aligned}
$$


in which $\nu_{i j}$ are the Poisson's coefficients in the $i$ - $j$ planes, $E_{i i}$ are the Young's moduli in $i$-direction and $G_{i j}$ are the shear moduli in the $i$ - $j$ planes. Also, Eqs. (2.13) and (2.14) puts in evidence where and how degradation will act during the progressive failure analyses, a feature to be better explained in the following sections.

Regarding the constitutive equations for multidirectional laminates, due to the existence of through-the-thickness stress gradients it is not convenient to write in the form of stress-strain relationships. Therefore, it is preferable to write the relationships between loadings (forces and moments) and stresses, i.e. to work with force $\underline{\mathrm{N}}$ and moment $\underline{M}$ resultants, which are obtained by integration of stresses over the laminate thickness $z$ by imposing static equilibrium condition. Also, considering a plane stress state in a lamina and the assumptions of the classical laminate theory (CLT), it follows that the strain-displacement relationships for the laminate are the same as those obtained by the Kirchhoff-Love (LOVE, 1888) plate theory. Figure 5 shows up the adopted convention for laminae numbering.

Figure 5: Lamina numbering system for a laminate.

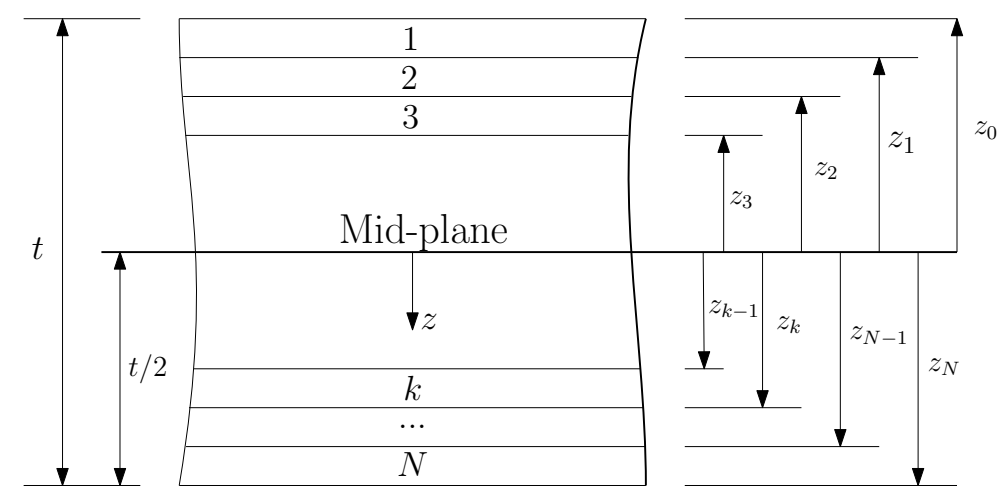

Source: adapted from (DANIEL; ISHAI, 1994) by the author.

So, the force and moment resultants (per unit length) acting at the mid-plane of the laminate are as follows:

$$
\begin{aligned}
& \underline{\mathrm{N}}=\int_{-t / 2}^{t / 2} \underline{\sigma} d z=\sum_{k=1}^{N} \int_{z_{k-1}}^{z_{k}} \underline{\sigma} d z, \\
& \underline{\mathrm{M}}=\int_{-t / 2}^{t / 2} \underline{\sigma} z d z=\sum_{k=1}^{N} \int_{z_{k-1}}^{z_{k}} \underline{\sigma} z d z,
\end{aligned}
$$

in which $\underline{\sigma}=\left\{\sigma_{x}, \sigma_{y}, \tau_{x y}\right\}^{T}, \underline{\mathrm{N}}=\left\{N_{x}, N_{y}, N_{x y}\right\}^{T}$ and $\underline{\mathrm{M}}$ is analogous. In terms of its components, $N_{x}$ and $N_{y}$ are the resultant of normal stresses, $N_{x y}$ is the in-plane shear stress resultant, $M_{x}$ and $M_{y}$ are the bending moment resultants and $M_{x y}$ is the torsional moment resultant, all of them per unit length. The stress-strain relations are given by,

$$
\underline{\sigma}_{k}=\underline{\underline{Q}}^{k} \underline{\varepsilon}^{0}+z \underline{\underline{Q}}^{k} \underline{\kappa}
$$


where $\underline{\varepsilon}^{0}$ and $\underline{\kappa}$ are the mid-plane strains (engineering) and curvatures, respectively. In the 2D case, the matrix $\underline{\underline{Q}}^{k}$ is the transformed reduced stiffness matrix, that depends of the orientation angle of each $k$-th lamina through the laminate and the engineering material properties. Now, substituting Eq. (2.15) in (2.17) and noticing that the mid-plane strains and curvatures are constant through the thickness it follows that,

$$
\begin{aligned}
& \underline{\mathrm{N}}=\left(\sum_{k=1}^{N} \underline{\underline{Q}}^{k} \int_{z_{k-1}}^{z_{k}} d z\right) \underline{\varepsilon}^{0}+\left(\sum_{k=1}^{N} \underline{\underline{Q}}^{k} \int_{z_{k-1}}^{z_{k}} z d z\right) \underline{\kappa}, \\
& \underline{\mathrm{M}}=\left(\sum_{k=1}^{N} \underline{\underline{Q}}^{k} \int_{z_{k-1}}^{z_{k}} z d z\right) \underline{\varepsilon}^{0}+\left(\sum_{k=1}^{N} \underline{\underline{Q}}^{k} \int_{z_{k-1}}^{z_{k}} z^{2} d z\right) \underline{\kappa} .
\end{aligned}
$$

Defining the extensional $\underline{\underline{\mathrm{A}}}$, coupling $\underline{\underline{\mathrm{B}}}$ and bending-torsion $\underline{\underline{\mathrm{D}}}$ stiffness matrices as,

$$
\begin{aligned}
& \underline{\underline{\mathrm{A}}}=\sum_{k=1}^{N} \underline{\underline{Q}}^{k}\left(h_{k}-h_{k-1}\right), \\
& \underline{\underline{\mathrm{B}}}=\frac{1}{2} \sum_{k=1}^{N} \underline{\underline{Q}}^{k}\left(h_{k}^{2}-h_{k-1}^{2}\right), \\
& \underline{\underline{\mathrm{D}}}=\frac{1}{3} \sum_{k=1}^{N} \underline{\underline{\underline{Q}}}^{k}\left(h_{k}^{3}-h_{k-1}^{3}\right),
\end{aligned}
$$

and substituting back in Eqs. (2.18) and (2.19) it results in the constitutive equations for multidirectional laminates which is given by,

$$
\left\{\begin{array}{l}
\underline{\mathrm{N}} \\
\underline{\mathrm{M}}
\end{array}\right\}=\left[\begin{array}{ll}
\mathbf{A} & \mathrm{B} \\
\mathbf{B} & \mathrm{D}
\end{array}\right]\left\{\begin{array}{c}
\underline{\varepsilon}^{0} \\
\underline{\kappa}
\end{array}\right\},
$$

where $\mathbf{A}=\underline{\underline{A}}$ for simplicity and applies analogously for the other two matrices. Notice that in the CLT approach the effect of transverse shear is not considered due to the kinematic assumptions and, geometrically, this means that the cross-sections transverse to the mid-plane remain plane and normal to it after the structure deforms. In cases where these effects are relevant, one needs to use other kinematic assumptions such as Mindlin-Reissner (REISSNER, 1945; MINDLIN, 1951), Reddy (REDDY, 2003) or Higher-Order Shear Deformation Theories (ABRATE, 1998), for example. Furthermore, it is important to highlight that for finite element analysis in which plate and/or shell elements are used these theories should be taken into consideration to allow obtaining the transverse shear stress distribution through-the-thickness of the laminate (ANGELO, 2018). One strategy described by Vinson and Sierakowski (VINSON; SIERAKOWSKI, 2006) considers that the strain field caused by the transverse shear is defined by a parabolic function in each layer being discontinuous at the interface. Therefore, the resultant of out-of-plane shear forces $Q$ can be calculated as,

$$
\begin{aligned}
& Q_{x}=2\left(A_{55} \varepsilon_{x z}+A_{45} \varepsilon_{y z}\right), \\
& Q_{y}=2\left(A_{45} \varepsilon_{x z}+A_{44} \varepsilon_{y z}\right),
\end{aligned}
$$


in which,

$$
A_{i j}=\frac{5}{4} \sum_{k=1}^{N} \bar{Q}_{i j}^{k}\left[h_{k}-h_{k-1}-\frac{4}{3}\left(h_{k}^{3}-h_{k-1}^{3}\right) \frac{1}{h^{2}}\right] .
$$

for $i, j=4,5$ and $h$ is the ply thickness.

\subsection{Impact on Composite Materials}

According to Abrate (ABRATE, 1998), laminated composite materials usually does not possess significant transverse strength. Thereby, structures composed by this kind of material are susceptible to be severely damaged after an impact event. Since there are various ways in which a structure can be inflicted by a foreign object, it is important to model its behavior in such events. Accordingly, there exists three principal types of models to describe impact mechanics which are:

- Energy-balance models that assumes a quasi-static behavior of the structure;

- Spring-mass models that accounts for the dynamics of the structure in a simplified manner;

- Complete models where the dynamic behavior of the structure is fully modeled.

In this context, an impact event involves the motion of the target and projectile. Also, the local indentation in the contact zone is relevant as well. All these characteristics are directly dependent of some physical quantities such as velocity and geometry of the impactor, constitution of the target, angle of incidence, to name a few. Thus, since these parameters affects the impact dynamics, several models have been proposed in the literature (ABRATE, 1998). Nevertheless, all these models have the common objective to predict the contact force history, extension of the damaged zone, estimation of the residual strength and the overall response of the structure.

Commonly, impacts are classified as low-velocity (LVI), high-velocity (HVI) or ballistic impacts. Among these, LVI has a particular importance since the structure presents usually only internal damage consisting mainly of delamination. However, the definition differs between authors. Liu and Malvern (LIU; MALVERN, 1987) classifies it according to the induced damage in the target. Meanwhile, Cantwell and Morton (CANTWELL; MORTON, 1991) classified low-velocity impact considering initial projectile velocities up to $10 \mathrm{~m} / \mathrm{s}$. By the other hand, Olsson (OLSSON, 2003) proposed for plates that the impact response is governed by the target-impactor mass ratio classifying the event as small and large mass impact as depicted in Figure 6.

In Olsson's work, small mass impacts occur when the target-impactor mass ratio follows Eq. (2.27), where the mass of the largest possible target area in which flexural waves 
Figure 6: Comparison between large (a) and small (b) mass impact.

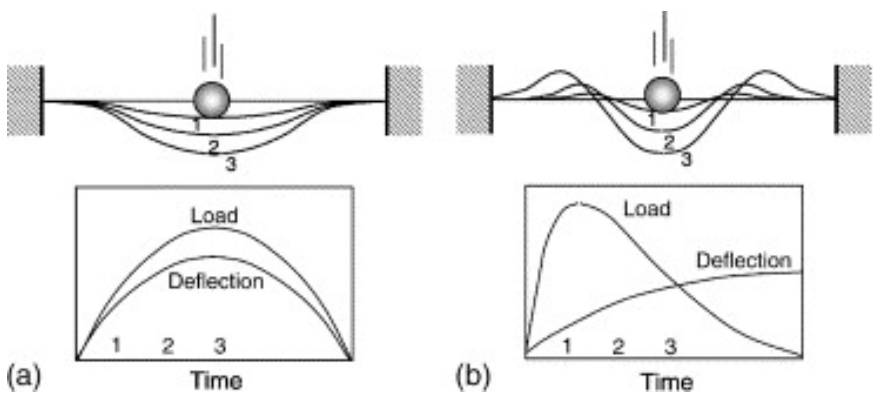

Source: after Olsson (OLSSON, 2003).

do not interfere with the boundaries is considered; large mass impacts occurs when the ratio follows Eq. (2.28). Considering a semi-spherical impactor geometry and a rectangular composite plate with length $a$ and width $b$, the criterion for small mass impact is

$$
M_{i} \leq \frac{M_{p}}{\sqrt{2 \pi}} \min \left[\frac{a}{b}\left(\frac{D_{22}}{D_{11}}\right)^{1 / 4}, \frac{b}{a}\left(\frac{D_{11}}{D_{22}}\right)^{1 / 4}\right]
$$

and, for large mass impact it follows

$$
M_{i} \geq 2 M_{p}
$$

where $M_{i}, M_{p}$ are the impactor and plate mass, respectively and $D_{i j}$ are the components of the bending-torsion stiffness matrix, written in the global coordinate system.

It can be well noticed in Figure 6 that for large mass impacts (heavy impactor in comparison with the target) load and deflection have the same behavior as under a static load being highly dependent of the boundary conditions. On the other hand, for small mass impacts, the response is governed by wave reflection from the boundaries. Thus, since it is a wave controlled local response, it does not depend of the boundary conditions. Moreover, there is also classifications of the impact phenomenon based on the duration of the contact interaction between target and indenter. The response illustrated in Figure 6 (a) is the focus of the present work being related with barely visible impact damage (BVID). That is, when impacted, the target does not present noticeable damage although its strength and stiffness are considerably affected.

Recently, in a review and benchmark study, a new method to classify the LVI is proposed by Bogenfeld, Kreikemeier and Wille (BOGENFELD; KREIKEMEIER; WILLE, 2018). It is based on the energy ratio between the first eigenmode and the total impact energy of the system. If the first eigenmode energy is at least equal to $98 \%$ of the total impact energy, it means that the system complies the assumptions of low-velocity impact. Therefore, this criterion is called Mode One Energy Ratio (MOER) and because of the aforementioned is also classified as an energy-based criterion. Moreover, it says 
that assuming Olsson's assessment of LVI, only the first mode is considered as damage relevant. Thus, the energy associated with other eigenmodes are not contributing to effectively increase damage. This makes clear that defining the impact event by means of the impactor mass or velocity does not take into account the structural response. Also, the study performed still defines a quantitatively limit for impactor speed which lies around 15 $\mathrm{m} / \mathrm{s}$ (which differs from the $10 \mathrm{~m} / \mathrm{s}$ limit proposed by Cantwell and Morton). Furthermore, this speed limit is associated with the damage pattern identified in the target. If it follows some damage patterns well described in the mentioned work and the MOER criterion, then the impact is within the limit of LVI. It is worth noting that this criterion is one of the few that considers both target and impactor physical characteristics to classify the impact event. While these factors are physically based, this criterion is a phenomenological one. Hereafter, this work use both this criterion and low-energy impact (LEI) terminology to precisely classify and refers to the impact phenomenon, respectively.

As discussed above, the literature has not yet reached to a complete clear definition of what a low-energy impact is. Thus, through this text it is also used an analytical strategy along with the MOER criterion to close even more the problem. As a matter of fact, this criterion is used to verify if there is the necessity of employing a quasi-static or a dynamic analysis approach, i.e. to know if the inertial terms can be neglected or not. In a series of works, Christoforou and Yigit (CHRISTOFOROU; YIGIT, 1998; CHRISTOFOROU, 2001; CHRISTOFOROU; ELSHARKAWY; GUEDOUAR, 2001; CHRISTOFOROU; YIGIT, 2009; YIGIT; CHRISTOFOROU, 2007) proposed the impact characterization diagram shown in Figure 7. Assuming the simplified models of infinite plate and quasi-static regime, this diagram predicts some important features of the phenomena for a reasonable range of impact cases. The diagram is divided in four regions. The transition regime encloses the region where a combination of both quasi-static and infinite plate behaviors is observed. In the quasi-static boundary curve and above it, the response is somewhat like the one illustrated in Figure 6 (a) and, obviously, quasi-static. Points that lies in or below the dashed line behaves with an infinite plate response and there is also a region next to the maximum dimensionless force $\left(\bar{F}_{\max } \approx 1.0\right)$ that results in the half-space behavior.

Since the present work deals only with impact cases in which the structure behaves in a quasi-static manner, it follows that the asymptotic limits presented in Figure 7 must be satisfied. The quasi-static behavior boundary is defined by,

$$
\bar{F}_{\max }=\sqrt{\frac{0.68}{0.68+\zeta_{w}}},
$$

where $\zeta_{w}$ is called loss factor and is a dimensionless parameter (CHRISTOFOROU; YIGIT, 1998). It follows that,

$$
\zeta_{w}=\frac{1}{16} \sqrt{\frac{K_{\alpha} M_{i}}{I_{1} D^{*}}},
$$


Figure 7: Impact Characterization Diagram.

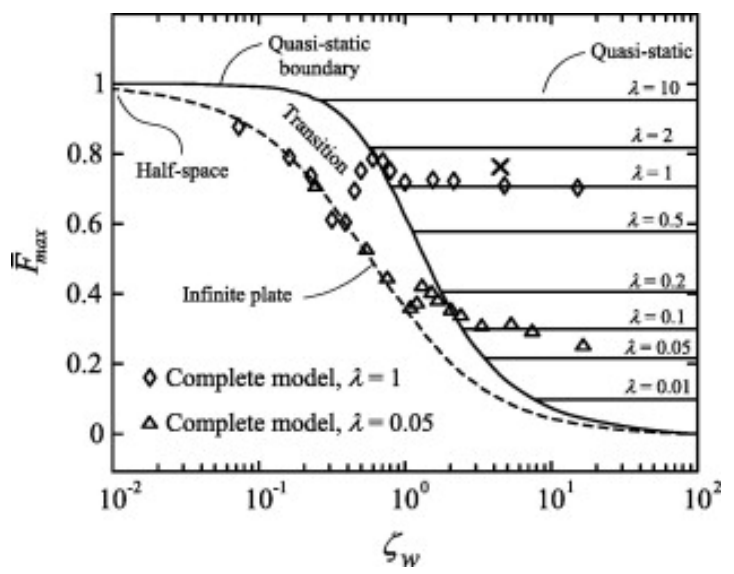

Source: after Sebaey (SEBAEY et al., 2013).

in which $K_{\alpha}$ is the contact stiffness, $M_{i}$ is the impactor mass, $I_{1}$ is the inertial parameter and $D^{*}$ is the plate effective bending stiffness (also with the reference notation conserved). Accordingly, the values of these parameters are given by

$$
\begin{aligned}
& K_{\alpha}=10.4 R_{i} S^{L}, \\
& I_{1}=\frac{M_{P}}{a b}, \\
& D^{*}=\frac{1}{2}\left(D_{12}+2 D_{66}+\sqrt{D_{11} D_{22}}\right),
\end{aligned}
$$

where $M_{p}$, a and b are the mass, length and width of the composite plate, respectively. Also, $R_{i}$ is the impactor radius whereas $S^{L}$ is the laminate in-plane shear strength (here the same notation used by Christoforou and Yigit is employed). The last can be experimentally obtained by tensile test in $\left[ \pm 45^{\circ}\right]_{n s}$ angle-ply laminates, for example. In the cases of LEI where Equation (2.28) is satisfied for large mass impacts, the normalized impact response is governed only by the parameter $\lambda$ named relative stiffness and with value defined by

$$
\lambda=\frac{K_{b s}}{K_{\alpha}}
$$

with $K_{b s}$ being the plate bending-shear stiffness calculated as

$$
K_{b s}=\frac{D^{*}}{0.0116 a^{2}}
$$

and finally, the normalized force is

$$
\bar{F}(\bar{t})=\sqrt{\frac{1+\lambda}{\lambda}} \sin \sqrt{\frac{1+\lambda}{\lambda}} \bar{t}
$$

where $\bar{t}$ is the normalized contact time defined as $\bar{t}=\omega t$ and with $\omega=\sqrt{\frac{K_{\alpha}}{M_{i}}}$ being the contact frequency. The maximum normalized impact force will be given by,

$$
\bar{F}_{\max }=\sqrt{\frac{1+\lambda}{\lambda}}
$$


with the corresponding normalized time,

$$
\bar{t}=\frac{2}{\pi} \sqrt{\frac{1+\lambda}{\lambda}}
$$

Therefore, from Equations (2.29) and (2.34) it follows that the limit of the quasi-static regime by means of the loss factor $\zeta_{w}$ and relative stiffness $\lambda$ is given by the relation,

$$
\zeta_{w} \geq \sqrt{\frac{0.68}{\lambda}}
$$

which along with the mode one energy ratio criterion is also a condition that should be satisfied to fulfill the assumption of low-energy impact.

\subsubsection{Impact Induced Damage}

Within the optics of LEI inducing barely-visible impact damage, this type of event should not result in penetration of the impactor on the target. BVID will be more explored in the next section of this manuscript. According to Abrate (ABRATE, 1998), experimental investigations indicates that the impact induced damage consists mainly of delamination, matrix cracking and fiber failure. Among these, delamination produces most significant strength reduction of the laminate and, in the range of BVID, it is only noticed between adjacent layers with different fiber orientations. Besides that, it is usually noticed that the delaminated area has a peanut shape with its major axis oriented at the same direction of the fibers in the lower ply at that interface as can be seen in Figure 8.

Figure 8: Delaminated area orientation and morphology.

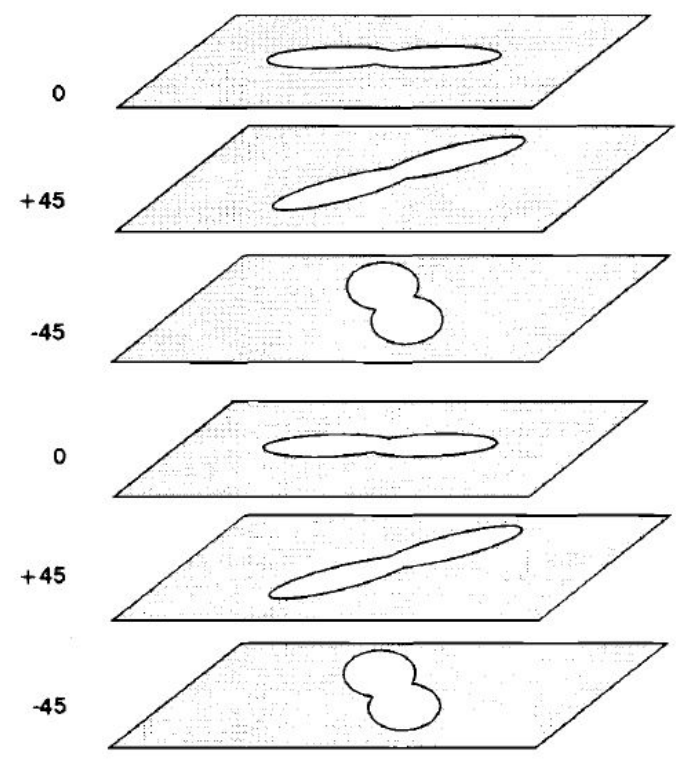

Source: after Abrate (ABRATE, 1998). 
Moreover, there is still a minimum energy level that the impact event has to reach to initiate delamination even in laminates with different fiber orientation through its layers. This energy is the initial kinetic energy of the impactor and when it is capable of induce delamination it is called by delamination threshold. There are several studies supporting that when the threshold energy is reached, the delamination size grows linearly with the kinetic energy of the impactor (ABRATE, 1998). As aforementioned, laminates subjected to low-velocity impacts presents also the failure modes of fiber fracture and matrix cracks as well as depicted in Figure 9.

Figure 9: Matrix and fiber failures.

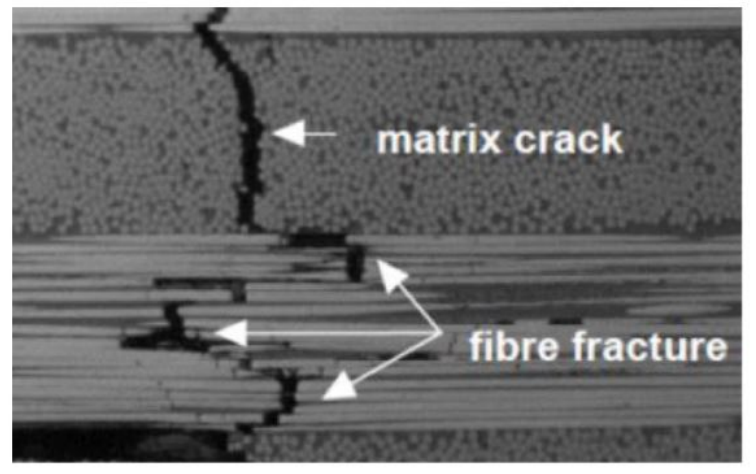

Source: after Thorsson, Waas and Rassaian (THORSSON; WAAS; RASSAIAN, 2018a).

The first does not influences the residual strength of the laminate significantly but it triggers delamination onset and propagation in impacted composite structures and can occur at the longitudinal, transverse or both directions. The process is initiated with some micro-defects, such as remaining voids from manufacture procedures, that are present in the structure. Thus, when impacted, these defects tend to concentrate stress that opens these cracks making them grow and coalesce giving rise to two types of matrix cracks that are the shear and tensile ones. Shear cracks are originated by high shear stresses and are positioned at an angle from the midsurface while tensile cracks are introduced when in-plane normal stresses exceed the transverse tensile strength of the ply (ABRATE, 1998). Figure 10 shows a schematic of both shear and tensile cracks.

When the first defect appears, it occurs a redistribution of the loading path that induces the appearance of more damaged areas. The crack tips meet the neighbor layers that can possess distinct fiber orientation promoting a huge growing in the shear stress; this is the onset of delamination. The number of delaminations increases in quantity and propagates when the applied loadings grow which can be easily observed in a 3-point bending test. Thus, when a laminate possesses layers with different fiber orientations this means that it possesses distinct bending stiffnesses which generates a high stiffness gradient through the thickness promoting dissimilar mechanical responses at each layer culminating 
Figure 10: Shear cracks (a) and tensile crack (b) scheme.

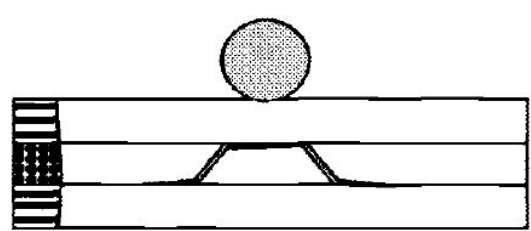

(a)

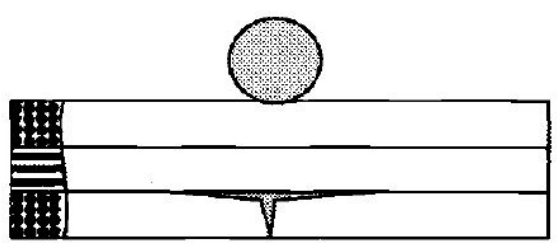

(b)

Source: after Abrate (ABRATE, 1998).

in a stress state favorable to the separation of these layers and this is all triggered by those matrix cracks. Therefore, in some sense, the failure mechanism of laminated composite materials is similar to the metals in respect to the process evolution: it initiates by small mechanisms (intralaminar damage) and progress to visible ones (interlaminar failure). Moreover, there is still the pine tree pattern that can be observed at impacted laminates (ABRATE, 1998). At thick laminates, matrix damage is localized at the same side in which impact has occurred and that is due to the high and localized action of the contact stresses between the impactor and the target resulting in a pine tree pattern. Analogously, for thin laminates, it occurs the opposite: the bending stresses in the bottom of the plate introduce matrix cracks firstly at the not impacted side of the coupon that at a final stage results in a reversed pine tree pattern as shown in Figure 11.

Figure 11: Pine tree (a) and reversed pine tree (b) patterns for thick and thin laminates, respectively.

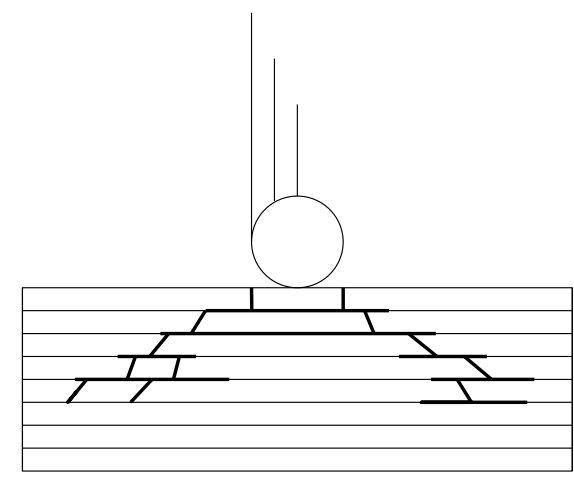

(a)

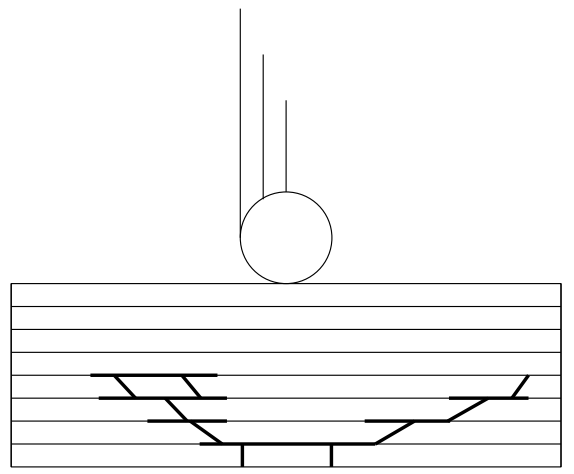

(b)

Source: adapted from (ABRATE, 1998) by the author.

During an impact event, fiber breakage can occur while increasing the kinetic energy level. Since it is the most energetic failure mechanism in composite materials, it will always show up after matrix cracking and delamination occurrence and it can be 
commonly found under the contact zone between the indenter and target. According to Nilsson (NILSSON, 2005), distribution of fiber breakage through-the-thickness is somewhat uniform for all laminates with a narrow distribution in the width and, under tensile stress, is the most important damage mode with the residual strength being driven by the level of occurrence of this mechanism. Regarding to matrix damage, it will highly influence the residual strength in the case of shear loading (SANDHU, 1976).

\subsubsection{Barely-Visible Impact Damage}

In the context of aeronautical industry, there are many primary aircraft structures made of composite materials. These, as already highlighted before, are very sensitive to impact induced damage. Thus, it is important to demonstrate that such structures are capable to possess residual strength even after the introduction of impact damage and, depending of the application, these structures must not have excessive deformations as well to ensure security and airworthiness. This must be true until the detection of the defect. After identification of damage, this is corrected by repairing the damaged region or substituting portions or even the whole part. Figure 12 shows up an example of one impacted composite plate (low-energy) and the damage left on both sides of it.

Figure 12: Example of damage left by LEI on a thin composite plate on both sides.

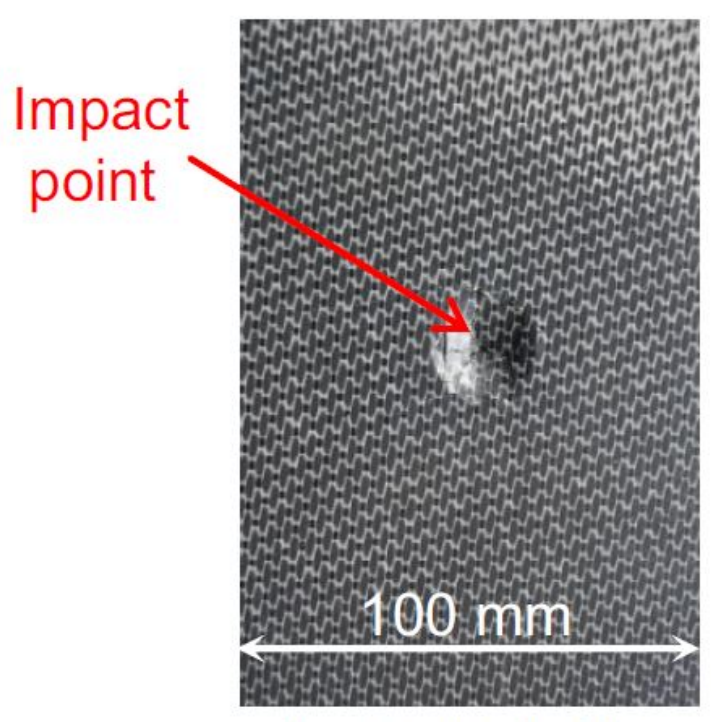

Impacted side

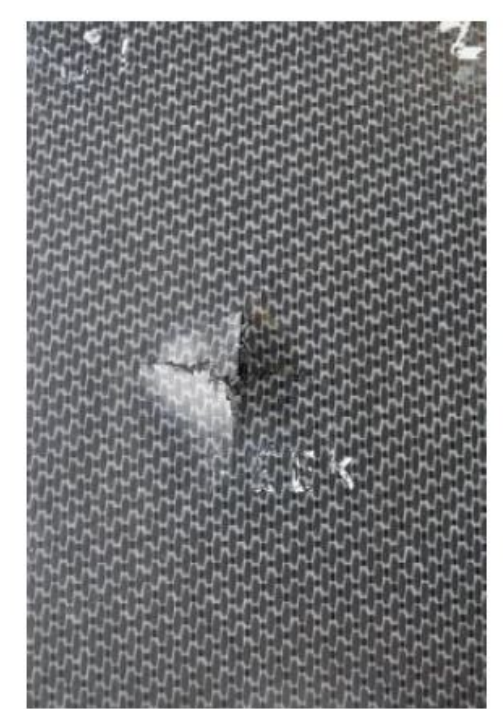

Non-impacted side

Source: after Bouvet and Rivallant (BOUVET; RIVALLANT, 2016).

Therefore, the application of the concept of damage tolerance for composite structures relies on two factors; lost of residual strength and damage detectability. Regarding the last one, for greater impact energies it is easier to identify the damage. Moreover, for aeronautic structures the damage is considered detectable only when it 
is visible at the impacted side of the structure since in most cases it is not possible to have access to the non-impacted side of those. So, for a damage tolerance design the main analysis parameter to be considered is the damage detectability and not the impact damage level, which can be counter intuitive. In this sense, an important curve to be analyzed is the residual strength as a function of the permanent indentation, as shown in Figure 13 for the case of compression-after-impact.

Figure 13: Compression residual strength as a function of the permanent indentation after impact.

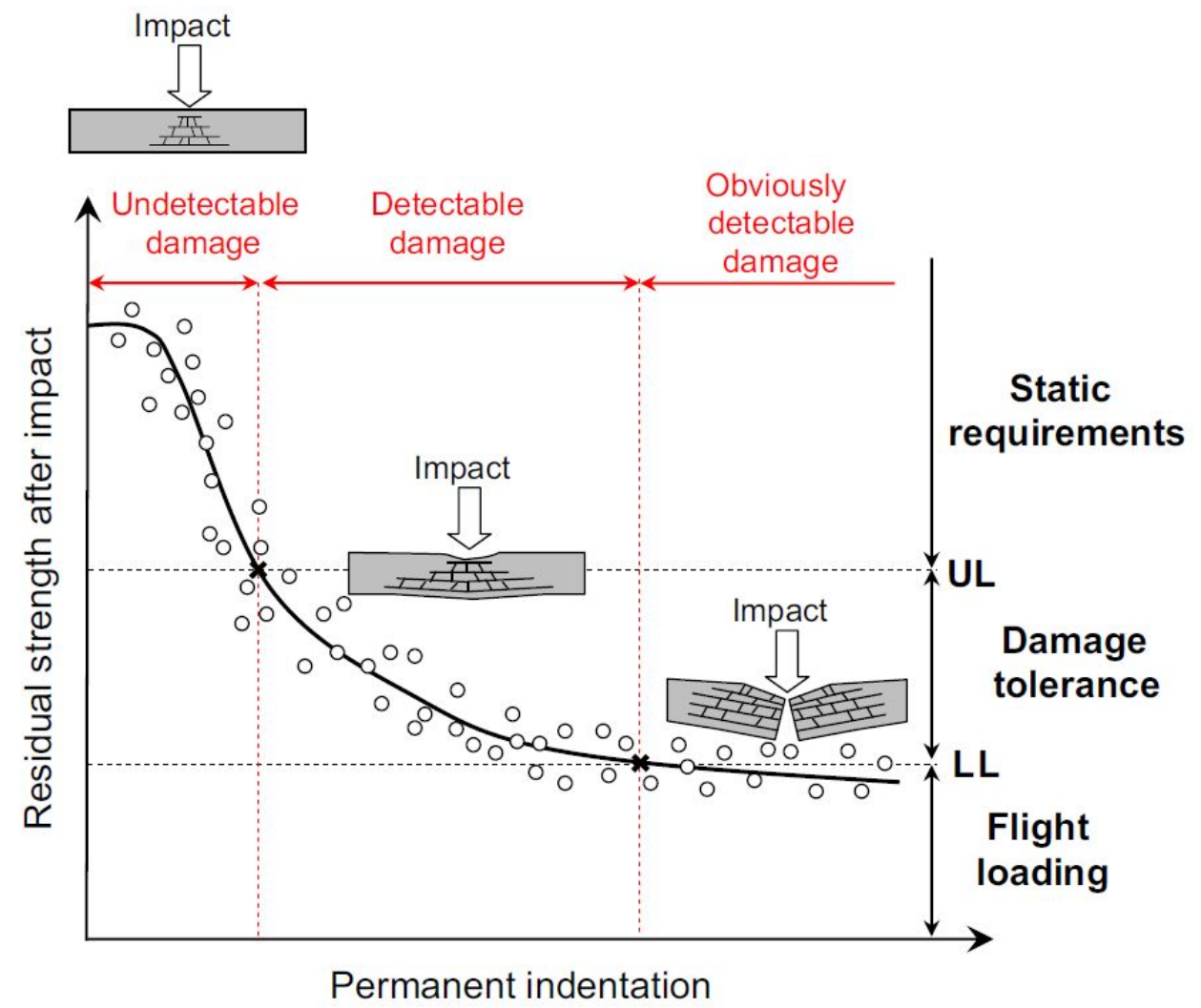

Source: after Bouvet and Rivallant (BOUVET; RIVALLANT, 2016).

In it, there are three regions classifying the damage extension. The first one is the region of undetectable damage in which the structure must withstand the static requirements until the ultimate load (UL). After it, there is the region of detectable damage (during inspection/maintenance) in which the structure has to sustain the limit load (LL). With the inspection procedures defined for damage detection, the structure is repaired and after it must withstand the ultimate load. Lastly, it is present the region of obviously detectable damage in which the structure must withstand loads ensuring safe flight conditions. As intuitive as it is, this kind of damage is the one that is readily detectable without any type of tool and deals with improbable impact events. Also, as in the previous cases, after repairing the structure, it must sustain the ultimate load. 
Still, as classified by the U.S. FAA in the AC 20-107B there are five categories describing damage in aeronautic composite structures according to its severity and detectability as shown in Figure 14.

Figure 14: Schematic of design load levels as a function of damage severity.

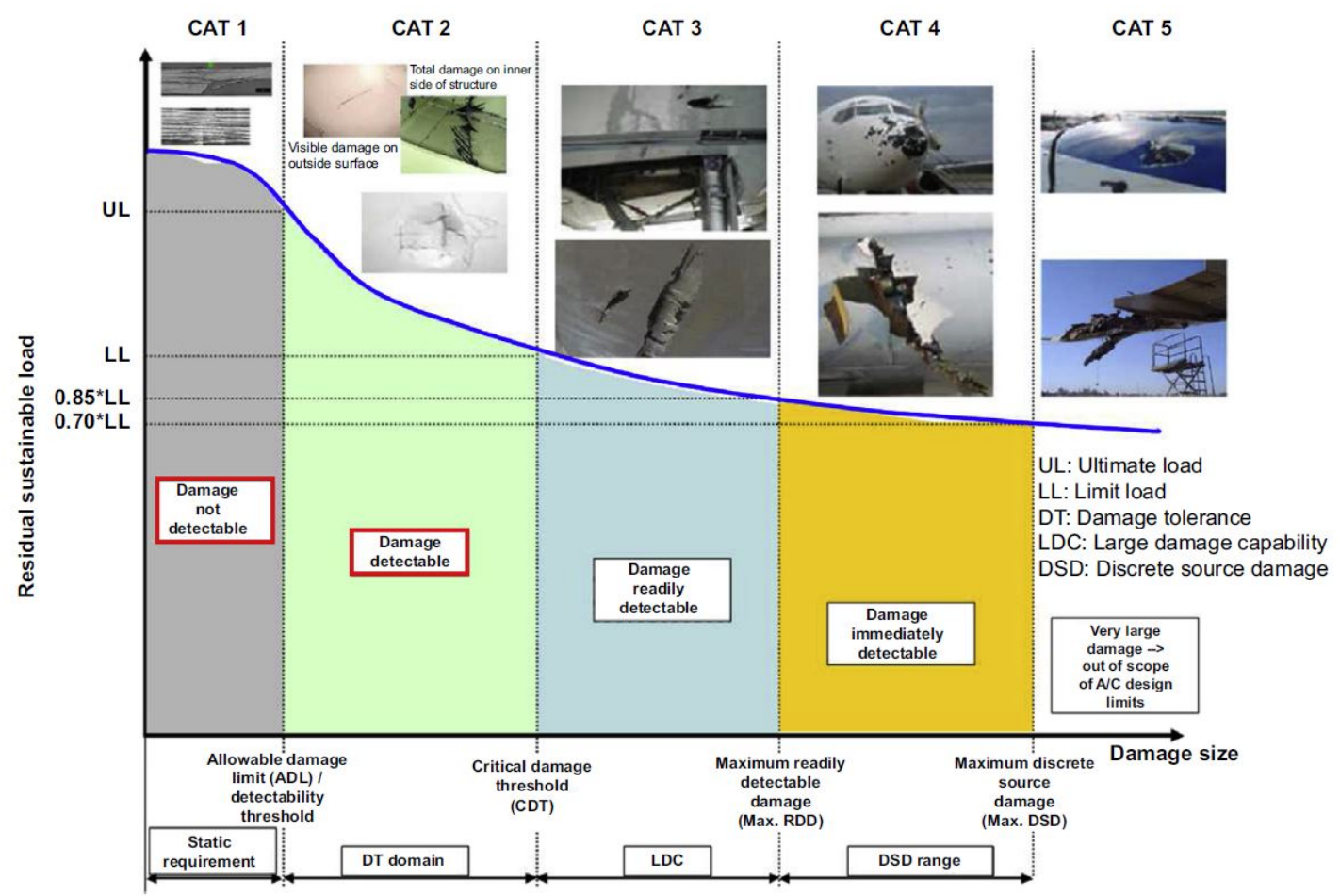

Source: adapted by Bouvet and Rivallant (BOUVET; RIVALLANT, 2016) from the FAA AC 20-107B.

Regarding the scope of this work, the category of damage of interest is the first one (CAT 1), which are those types of damages that can go unnoticed during scheduled or unscheduled inspections. Such structures must be reliable during service being capable of withstanding the ultimate load even with these damages; with the most important type and concern being the so called barely-visible impact damage (BVID). In this context, it is important to highlight the connection between BVID and LEI, with the prior being representative, for example, of tools dropping during maintenance and/or manufacture. The BVID, again, is very penalizing for the structure since it often goes unnoticed being able to induce matrix cracking, fiber failure at the impact region and delaminations in stacking sequences with high trough-the-thickness stiffness gradient.

The detectability of damage depends on the type of inspection method employed. There are four levels of inspection methods, defined as: walk-around, general visual inspection, detailed visual inspection and special detailed inspection. These are respectively placed from smaller to bigger level of detailing. The detailed visual inspection is the one to be focused here, since this is the one type in which the minimum detectable damage 
size is what is called by BVID. In it, the search of damage caused by impact and other abnormalities is carried out with the aid of appropriate lighting, magnifying glasses, surface cleaning and mirrors to name some of the most used tools. Thus, there are some detectability thresholds defined by the resolution of the employed inspection method. So, referring to Figure 15, the indentation depth is the parameter to be identified during inspection to define a BVID.

Figure 15: Damage size and inspection type dependency.

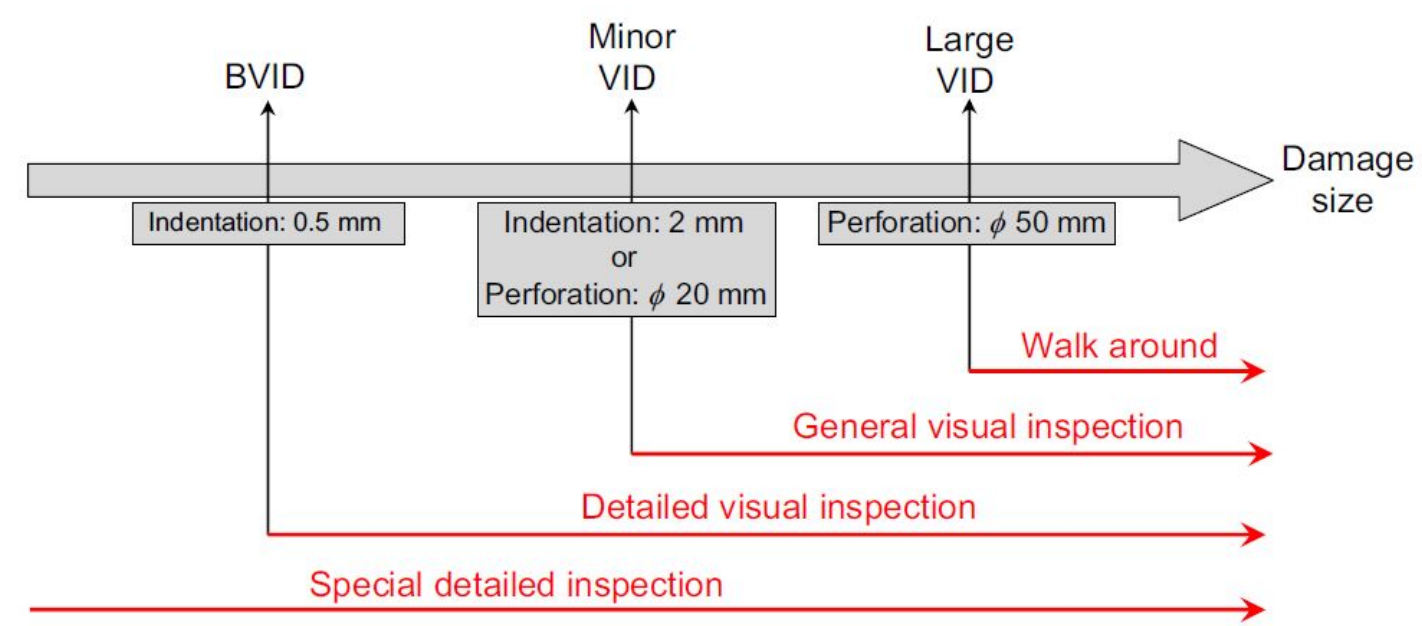

Source: after Bouvet and Rivallant (BOUVET; RIVALLANT, 2016).

It is important to highlight that for indentations between 0.25 and $0.5 \mathrm{~mm}$ the damage detectability employing a detailed visual inspection has a probability greater than 90\% (BOUVET; RIVALLANT, 2016). Therefore, in the present work the BVID is defined as any type of damage detectable by this type of inspection method with depths until 2.0 mm (without perforation).

\subsection{Damage, Degradation and Failure of Composite Materials}

The definition of failure changes from one application to another. It does not necessarily means that the structure is no more capable of endure loading in the sense that it suffers irreversible catastrophic damage. For example, in some cases, failure can stand for great displacements at some structure meaning that it suffered higher levels of it than the acceptable as design parameter. In other cases, it can mean the complete separation and/or fracture of the constituents.

For composite materials there are two possible paths to approach failure, namely First Ply Failure (FPF) and Last Ply Failure (LPF). In most cases, damage is non-uniform across the structure and it initiates before any changes in the physical appearance and/or on the macroscopic mechanical behavior of the structure. Generally, it occurs some fiber breakage and matrix cracks in some ply before than in another. In this manner, failure (in 
the sense of decrease of mechanical response capability) of a composite material occurs when one or more plies collapse. Thus, definition of which criterion and design philosophy is more suited to a specific situation is in the hands of the competent engineer.

Several theories have been developed during the last decades with various distinct approaches to failure. Then, it is straightforward to classify these lamina failure theories in categories, that are: limit or non-interactive, interactive and failure modes based theories (DANIEL; ISHAI, 1994). In the first type, either the stress or strain values (in local coordinate system) are compared with the corresponding allowable (or limit) values meaning that there are no interactions between stress and strain components (e.g. maximum stress and strain failure criteria). Interactive theories tends to include all stress components in a single expression to evaluate occurrence of failure and, thus, there is no distinction among failure modes (e.g. Hoffman and Tsai-Wu failure criterion). Finally, the last kind of theory which is mode based defines a different failure criterion for each of these failure modes (e.g. LaRC03 and Puck failure criteria).

For engineering purposes, there are some important aspects that any material model has to possess. It has to require simple tests for model parameters identification and to be easy for coupon manufacturing. Along with these, it is interesting that it can be implemented as a routine with ease possessing low computational cost (RIBEIRO, 2013). Likewise, aiming a low-cost design, it is usually recommended to choose a LPF philosophy as well. In these, failed ply loads are redistributed to the other laminate plies. Besides that, it is important to highlight the mechanisms that leads to failure in composite materials are not fully understood yet (DAVILA; CAMANHO; ROSE, 2005). This is even more visible in fiber and matrix compression failure cases. Due to these inherent challenges, it were realized two editions of the World-Wide Failure Exercises (WWFE) until the date of this work aiming the comparison and evaluation of the existent failure theories (HINTON; KADDOUR; SODEN, 2004; KADDOUR; HINTON, 2013). A large benchmark study is conducted to assess which theory best fits to the collected experimental data and they are ranked accordingly.

Degradation laws are those that will govern the damage evolution until failure of the composite laminate. Thus, when damage is detected by some failure criterion the damage evolution law will rule its propagation by reducing the laminate mechanical properties. Hahn and Tsai (HAHN; TSAI, 1974) presents in their work a brief general discussion of how property degradation should occur in composite materials as depicted in Figure 16.

Accordingly, composites with fibers oriented at $90^{\circ}$ should behave following the path D in Figure 16 presenting an abrupt decrease of mechanical properties. Hybrid composites tend to behave following the curve C or L. Lastly, for conventional cross-ply composite materials (i.e. with glass or carbon fiber reinforcement), the behavior follows the $\mathrm{G}$ line, meaning a gradual degradation process. Furthermore, degradation laws can be 
Figure 16: Properties degradation as proposed by Hahn and Tsai.

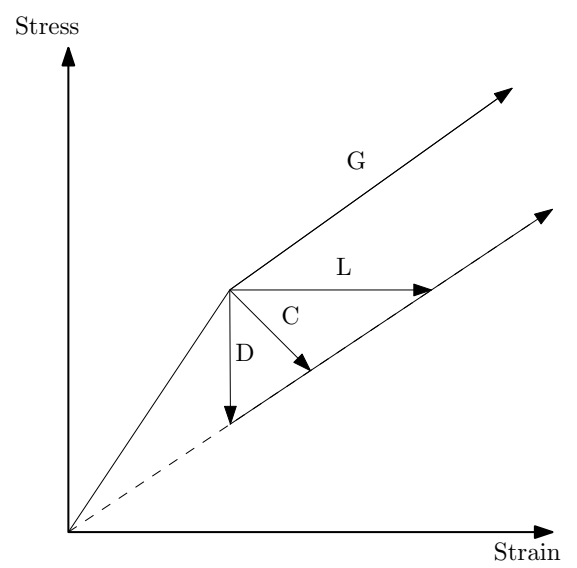

Source: adapted from (HAHN; TSAI, 1974) by the author.

mode dependent also and the properties can decay through a degradation function or a degradation factor. For example, Tita (TITA; CARVALHO; VANDEPITTE, 2008) uses both approaches. The longitudinal elastic modulus $\left(E_{11}\right)$ decays as

$$
E_{11}^{\mathrm{df}}=E_{11} \exp \left[-\left(\frac{A}{A_{0}}\right)\right]
$$

where $E_{11}{ }^{\text {df }}$ is the degraded elastic modulus, $A$ is the damaged zone (predicted by the failure criterion) and $A_{0}$ is the interaction failure zone of the fiber associated with the tensile resistance of it. The degradation law for the out-of-plane elastic modulus $E_{33}$ is given by

$$
E_{33}^{\text {dela }}=d_{1}^{\text {dela }} E_{33}
$$

with $d_{1}{ }^{\text {dela }}$ assuming a discrete continuous value of 0.01 when delamination is detected. Thus, it reduces abruptly by $99 \%$ the value of the elastic modulus as the failure occurs.

Due to the inherent anisotropy and heterogeneous nature of composite materials, describing its failure modes is a difficult task. Also, evolution of damage is not modeled with ease since intralaminar mechanisms interact between them. Still, the manufacture method highly influences the composite final quality being related with its reliability which implies in the usage of great safety margins resulting in conservative design draw-backs (TITA, 2003). However, besides all difficulties, composites had proven to be appliable engineering materials being used in several high-performance applications. Therefore, knowledge of its failure modes and mechanics is of fundamental relevance. According to Anderson (ANDERSON, 2005), there are five common types of intralaminar damage/failure mechanisms as represented in Figure 17.

Mechanism 1 is known as pull-out and consists of the fiber rupture and its separation from the matrix. Before its occurrance, the fiber-bridging (mechanism 2) can take place if 
Figure 17: Intralaminar failure modes.

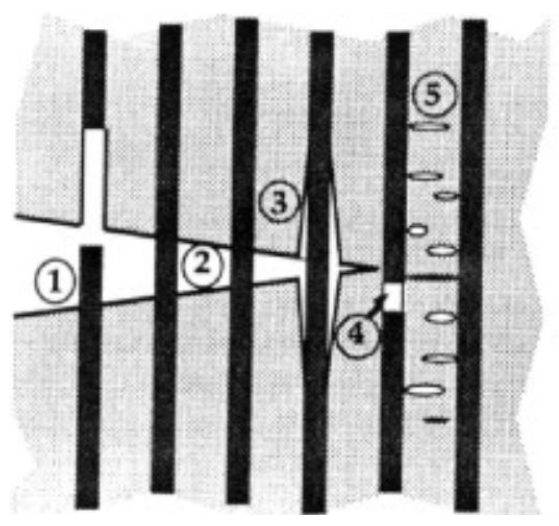

Source: after Anderson (ANDERSON, 2005).

the fiber-matrix interface resistance is high. Moreover, debonding failure can show up when there is weak fiber-matrix interaction as illustrated by mechanism 3. The fourth mechanism consists basically on the fiber fracture itself characterized by high energy release. Finally, damage inside the matrix continuous phase can occur, promoting stress concentrations and failure a posteriori. All those mechanisms depends on the loading nature. For example, under compression a fiber reinforcement can present micro-buckling promoting instability (either in extension or shear modes) of the structure. Along with this, compression loadings can induce failure by the mechanism of fiber kinking. This occurs due to the existence of fiber misalignment regions and the damage, mainly due to shear stresses, promoted in the supporting matrix as illustrated in Figure 18.

Figure 18: Kink band region (left) and fiber misalignment represented by local waviness (right) at some lamina.
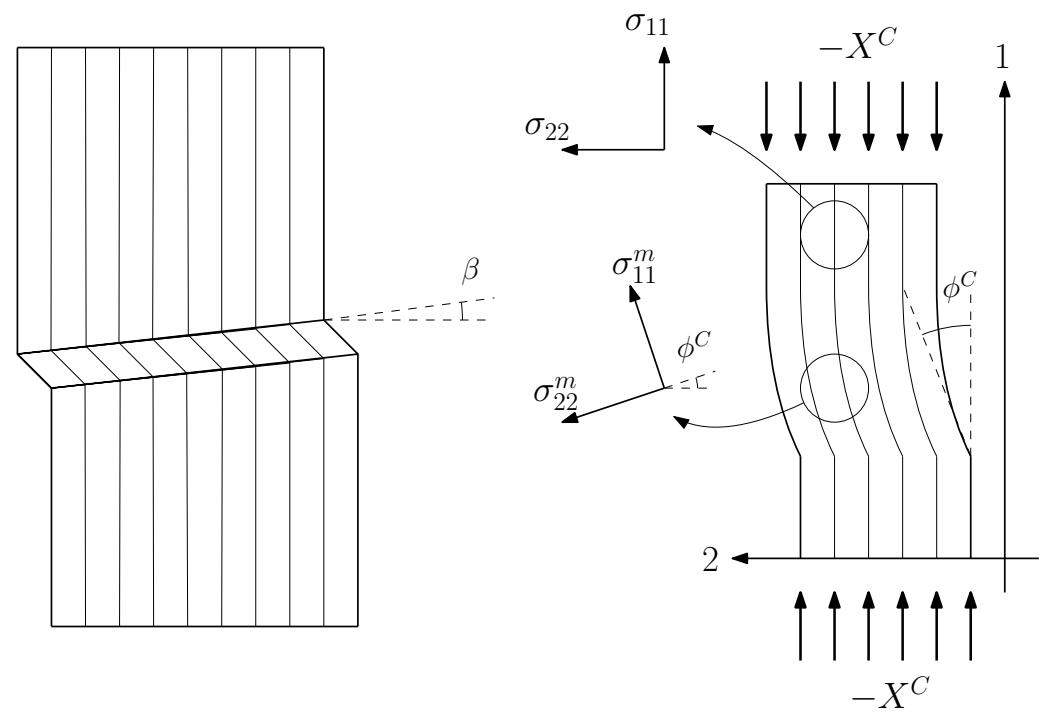

Source: adapted from (PINHO et al., 2005) by the author in (SOUZA, 2017). 
Also, matrix damage is directly related to its ductility and the in-service or imposed temperature, being possible to present a brittle or an inelastic behavior. Moreover, a composite structure can be subjected to different kinds of loadings such as shear and transverse compression simultaneously. Accordingly, an unidirectional (UD) composite lamina will present a fracture plane with some angle $\alpha$ in respect with the 1-2 plane (Figure 19). For example, Puck's failure criteria (PUCK; SCHÜRMANN, 2004) is able to capture this feature and is based on the actuation plane theory.

Figure 19: Fracture of a UD lamina submitted to transverse compression and in-plane shear.
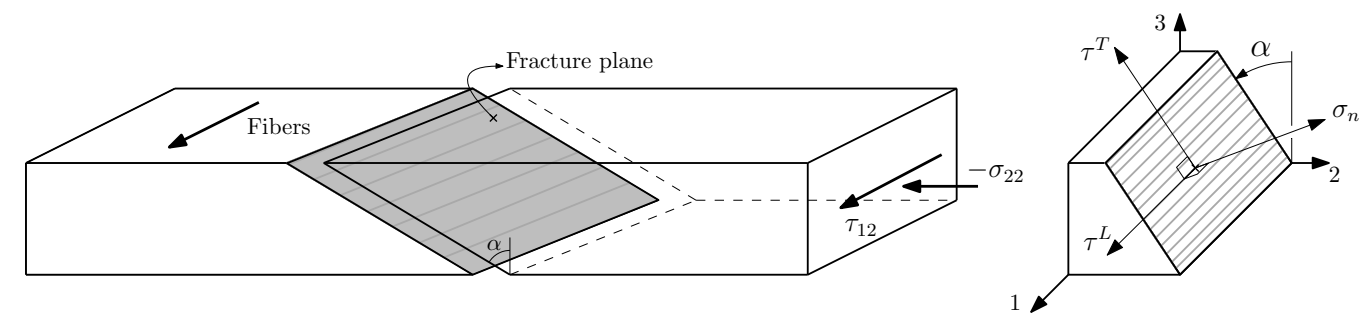

Source: adapted from (DAVILA; CAMANHO; ROSE, 2005) by the author in (SOUZA, 2017).

\subsubsection{Continuum Damage Mechanics}

Continuum damage mechanics development started in 1958 with Kachanov's work (KACHANOV, 1958) on the weakening of metals due to the deterioration process where a changing in rupture characteristic from ductile to fragile was noticed. Also, a one-dimensional (1D) surface damage variable and an isotropic treatment to damage is employed for this purpose. A more rigorous approach using a thermodynamic treatment to damage mechanics making the foundations for the justification of CDM as a theory was developed by the end of the 1970s and beginning of the 1980s in the works by Chaboche (CHABOCHE, 1978), Chaboche and Lemaitre (CHABOCHE; LEMAITRE, 1978), Murakami (MURAKAMI, 1981), Cordebois and Sidoroff (CORDEBOIS; SIDOROFF, 1982), Krajcinovic and Fonseka (KRAJCINOVIC; FONSEKA, 1981) and Krajcinovic and Silva (KRAJCINOVIC; SILVA, 1982). This enabled the application of CDM for solving engineering problems and it was based on the deterioration of mechanical properties through the classical concept of internal state variables and the basic principle of homogenization of a crack. As an example of engineering application, Lemaitre and Chaboche (CHABOCHE, 1981; LEMAITRE; CHABOCHE, 1985) use Kachanov's methodology applying it for several structural problems (e.g. turbine blade refractory alloys) and are based on previously developed concepts of effective stress (RABOTNOV, 1969) and strain equivalence. The last is an empirical postulated principle that, in the words of Lemaitre (LEMAITRE, 2012), means: "any strain constitutive equation for a damaged material may be derived 
in the same way as for a virgin material except that the usual stress is replaced by the effective stress".

Regarding to internal variables, it represents, directly or indirectly, the density and/or distribution of microscopic defects that characterize damage (PINHO; IANNUCCI; ROBINSON, 2006b). Also, since damage is an irreversible process, any CDM model has to follow the Clausius-Duhem inequality (LEMAITRE, 2012) so that the second law of thermodynamics is satisfied.

According to Lemaitre (LEMAITRE, 2012), damage is the progressive physical process by which a material breaks. In a phenomenological sense, it is the study, through mechanical variables, of the deterioration that such material presents when subjected to some loading and its evolution by means of damage accumulation. This deterioration translates on reduction of the capability of supporting loads by reduction of stiffness and strength. This, at the microscale, is the accumulation of micro-stresses in the neighborhood of defects or interfaces and the breaking of atomic bonds. At the mesoscale, it manifests as the coalescence of microcracks or microvoids that, once together, initiates a crack. Finally, within macroscale optics it is simply the growth of that crack where, in a final stage, culminates in rupture. Generally, the last scale is approached using fracture mechanics concepts and the first two scales are studied using damage variables of CDM defined in the mesoscale as illustrated in the scheme of Figure 20.

Figure 20: Schematic representation of crack initiation and scales of analyses.

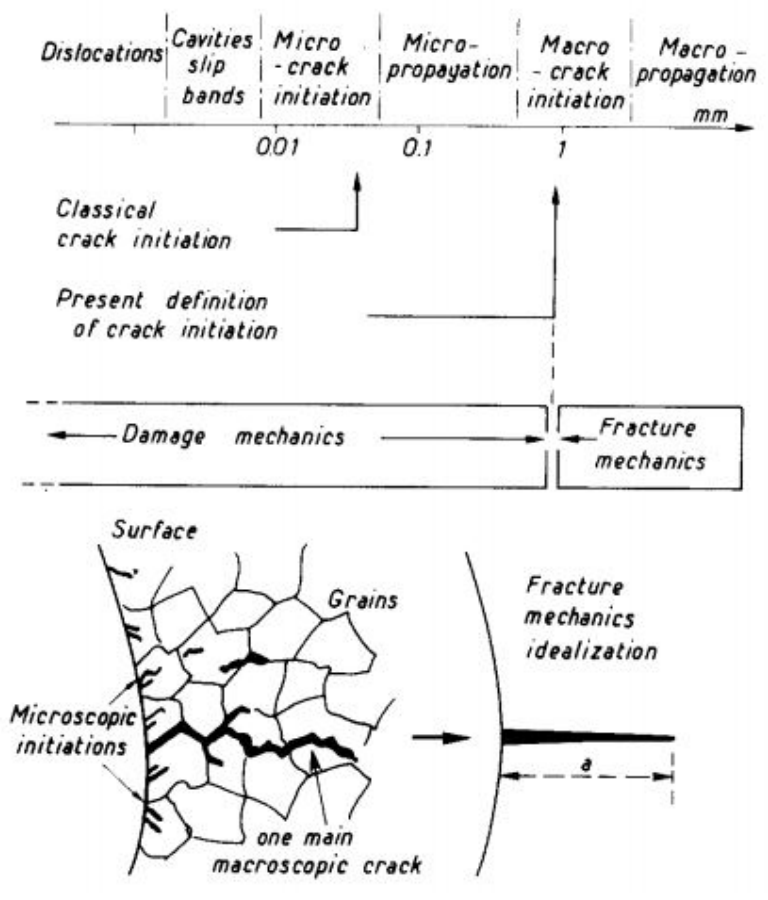

Source: after Chaboche (CHABOCHE, 1981).

Still, Lemaitre (LEMAITRE, 2012) supports that materials with distinct physical 
structures possess very similar qualitative mechanical behavior. Along with this, Tita (TITA, 2003) supports that the damage onset and progression on composite and metallic materials seems to be quite alike. Thus, this turns on possible the usage of common energy mechanisms concepts to describe mesoscopic properties of these materials. Since energy is a physical quantity that always search for restoration of perturbed states (i.e. pursuit for equilibrium states) acting in an unidirectional sense being responsible for the connection between cause and effect of phenomena, it is possible to explain material deterioration behavior using the laws of thermodynamics and mechanics of continuous media. So, continuous damage mechanics does not aim to account for the complexity of the microstructure of any kind of engineering material. Moreover, Lemaitre (LEMAITRE, 2012) list the existing manifestations of damage as follows:

- Brittle damage: occurs when a crack initiates at the lamina level without considerable plastic strain;

- Ductile damage: occurs when the nucleation and propagation of cavities progresses as result of plastic strains higher than a certain threshold value;

- Creep damage: occurs in metals loaded at elevated temperatures with plastic strains possessing a viscosity behavior (i.e. material may be deformed at constant stress). When strains are large enough, intergranular decohesions produces damage and an increase at the strain rate is noticed;

- Low cycle fatigue damage: occurs when a material is loaded cyclically at high values of stress and strain;

- High cycle fatigue damage: occurs under low levels of cyclic loadings bellow a reference stress value.

Also, Kachanov (KACHANOV, 2013) presents other types of damage manifestations that are chemo-mechanical and environmental damages, embrittlement of steel and damage of concrete. It is important to highlight that CDM deals with quantities in a mathematical sense, based on the assumption of continuity. Physically, this means that these quantities represent average values over a Representative Volume Element (RVE). According to Talreja (TALREJA, 2008), a composite under a deterioration process can be treated as a stationary structure consisting of fibers, particles and other constituents inserted in the matrix and a transient microstructure under an evolution process consisting of damage entities such as voids and cracks as depicted in Figure 21.

Within the CDM approach, homogenization is achieved proceeding the following two steps: 
Figure 21: Continuum damage mechanics concept for composite materials.

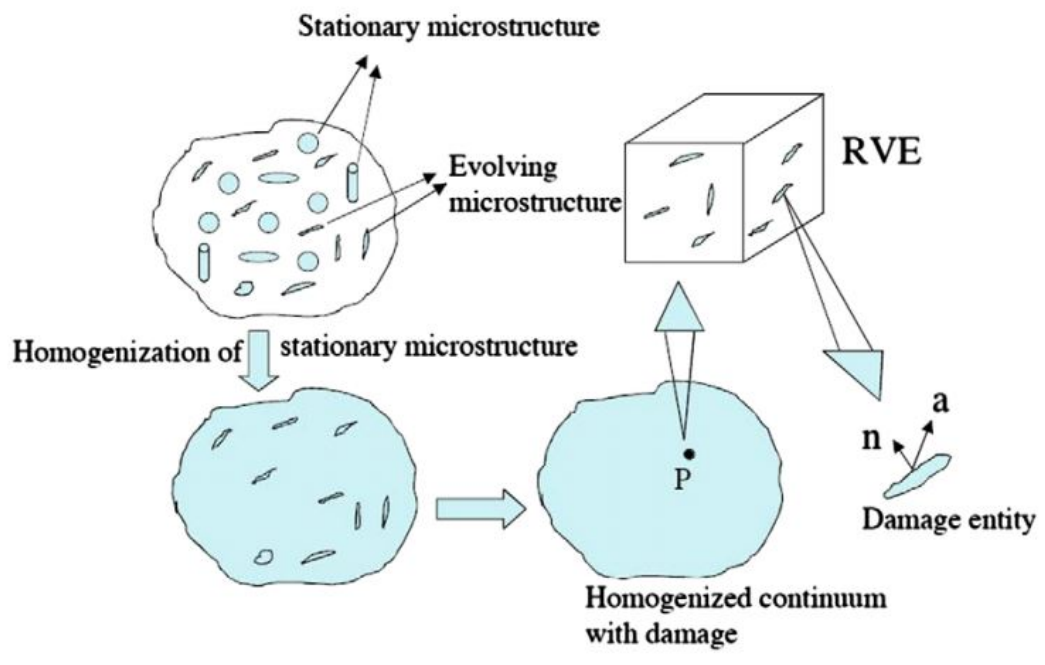

Source: after Talreja (TALREJA, 2008).

1. The stationary microstructure is transformed in a continuous media with anisotropic properties;

2. The microstructure under the evolution process is homogenized and substituted by a representative field of internal variables.

Consider a RVE including a point $\mathrm{P}$ where the composite is homogenized as illustrated in Figure 21. This volume allows a non-local description of the material state at the point $\mathrm{P}$. Thus, the stress, strain, temperature and internal variables are average values over the RVE. Also, the size of the RVE represents the mesoscale while voids and cracks are at the microscale level (TALREJA, 2008).

Continuum damage mechanics classical state laws and constitutive equations are derived postulating the existence of energy potentials. Thus, provided with the RVE in Figure 22 and homogenization concept, it is possible to derive analytical expressions accounting for the damage process.

At the RVE, damage can be defined as the ratio between the surface damaged area $\delta S_{\mathrm{D} x}$ and surface total area $\delta S$ as,

$$
D(\vec{n}, x)=\frac{\delta S_{\mathrm{D} x}}{\delta S}
$$

where $D(\vec{n}, x) \in[0,1]$ is defined as the dimensionless damage variable. Also, $\vec{n}$ is the normal vector defining the plane with abscissa $x$ along $\vec{n}$-direction. When $D=0$ the material is in its pristine form and when $D=1$ it is fully damaged (KACHANOV, 1958). Thus, damage can be interpreted as the effective surface density of microdefects. Though, 
Figure 22: Representative volume element with damage.

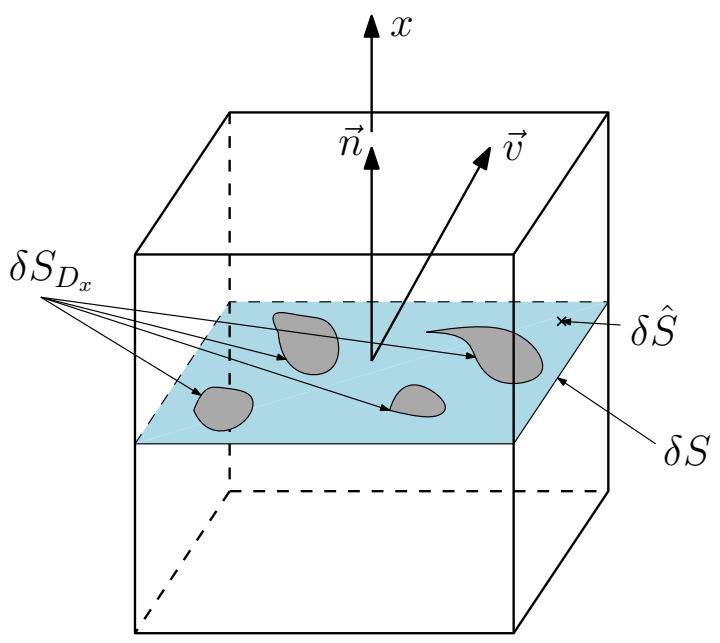

Source: adapted from (LEMAITRE, 2012) by the author.

damage is usually non-isotropic (LEMAITRE; DESMORAT, 2005) and, regarding the reference vector $\vec{v}$ in Figure 22, a more general definition of damage using tensors in index notation is,

$$
\left(I_{i j k l}-D_{i j k l}\right) v_{k} n_{l} \delta S=v_{i} \hat{n}_{j} \delta \hat{S}
$$

with $\hat{n}$ being the effective normal vector of the effective area $\delta \hat{S}=\delta S-\delta S_{\mathrm{D} x}$. Also, $I_{i j k l}$ and $D_{i j k l}$ are the identity and damage fourth-order tensors, respectively. The damage tensor is the operator which transforms $v_{i} n_{j} \delta S$ from the reference (or initial) to the $v_{i} \hat{n}_{j} \delta \hat{S}$ effective (or current) configuration and it has the following symmetries: $D_{i j k l}=D_{i j l k}=D_{j i k l}=D_{k l i j}$. The effective stress second-order tensor $\hat{\sigma}_{i j}$ is a projection of the initial stress tensor $\sigma_{i j}$ on the reference $\vec{v}$ vector direction in Figure 22. Thus, it is possible to write,

$$
v_{i} \hat{\sigma}_{i j} \hat{n}_{l} \delta \hat{S}=v_{i} \sigma_{k l} n_{j} \delta S
$$

and, substituting Eq. (2.41) in Eq. (2.42), it follows that,

$$
\hat{\sigma}_{i j}\left(I_{i j k l}-D_{i j k l}\right) v_{k} n_{l} \delta S=\sigma_{k l} v_{k} n_{l} \delta S
$$

Provided with the symmetry properties of the damage tensor, it follows that the effective stress tensor can be written as,

$$
\hat{\sigma}_{i j}=\sigma_{k l}(I-D)_{k l i j}^{-1},
$$

which is a symmetric tensor (LEMAITRE; DESMORAT, 2005). It is important to highlight that exists uniqueness of the fourth-order damage tensor that can fulfill the following restrictions: 
- Symmetry of the effective stress;

- Independence of the effective stress with the strain behavior and Poisson's effect;

- Existence of strain potentials and validity of the principle of strain equivalence which implies in compatibility with the thermodynamics framework;

- Different effects of the damage on the hydrostatic and deviatoric stress portions.

According to Lemaitre and Desmorat (LEMAITRE; DESMORAT, 2005), there is another way of defining the damage tensor which is through the initial elasticity tensor $E_{r s k l}$. Thus, the transformation operation will change this tensor into the current elasticity tensor $\hat{E}_{i j k l}$ softened by damage and is given by,

$$
\left(I_{i j r s}-D_{i j r s}\right) E_{r s k l}=\hat{E}_{i j k l} \text {. }
$$

It is worth noting that in a theoretical point of view, this definition does not yield a real state variable because it demands the previous knowledge of elasticity behavior (LEMAITRE; DESMORAT, 2005). Consider the fourth-order compliance tensor $S_{i j k l}=E_{i j k l}^{-1}$, with the last being previously denoted as the tensor $C_{i j k l}=E_{i j k l}$, and the complementary energy density for anisotropic elasticity given as,

$$
\mathrm{w}_{e}=\frac{1}{2} S_{i j k l} \sigma_{i j} \sigma_{k l} \text {. }
$$

In the case of composite materials, the damage mechanisms under shear and tension are different, thus, it is a good strategy to define two distinct scalar variables to govern this influence on elastic shear and hydrostatic energies. Such variables are $D_{s}$ for the deviatoric stress components and $D_{n}$ for hydrostatic stress components. Now, Eq. (2.46) can be rewritten as,

$$
\mathrm{w}_{e}=\underbrace{\frac{1}{2} S_{i j k l} \sigma_{i j}^{D} \sigma_{k l}^{D}}_{\text {shear energy }}+\underbrace{\frac{1}{2} S_{i j k l} \sigma_{H}^{2} \delta_{i j} \delta_{k l}}_{\text {hydrostatic energy }},
$$

in which $\sigma_{i j}^{D}$ and $\sigma_{H}$ are, respectively, the deviatoric and hydrostatic stresses and $\delta_{i j}$ is the Kronecker delta. By the definitions of stress deviator and hydrostatic stress, it follows that,

$$
\begin{aligned}
\sigma_{H} & =\frac{1}{3} \sigma_{k k}, \\
\sigma_{i j}^{D} & =\sigma_{i j}-\sigma_{H} \delta_{i j},
\end{aligned}
$$

and, when damage occurs in all directions, usage of the previous defined scalar variables is needed. Finally, the complementary energy density for damage in anisotropic materials is written as,

$$
\mathrm{w}_{e}=\frac{1}{2} S_{i j k l}\left[\frac{\sigma_{i j}^{D} \sigma_{k l}^{D}}{1-D_{s}}\right]+\frac{1}{2} S_{i j k l}\left[\frac{\sigma_{H}^{2} \delta_{i j} \delta_{k l}}{1-D_{n}}\right] .
$$


Provided with Eq. (2.49) it is now possible to determine elasticity law by,

$$
\begin{aligned}
\varepsilon_{i j}^{e D} & =\frac{\partial \mathrm{w}_{e}}{\partial \sigma_{i j}^{D}}, \\
\varepsilon_{H}^{e} & =\frac{\partial \mathrm{w}_{e}}{\partial \sigma_{H}},
\end{aligned}
$$

where $\varepsilon_{i j}^{e D}$ and $\varepsilon_{H}^{e}$ are the deviatoric and hydrostatic elastic strains, respectively. Also, it follows that the strain energy density release rate is,

$$
\begin{aligned}
Y_{s} & =\frac{\partial \mathrm{w}_{e}}{\partial D_{s}}, \\
Y_{n} & =\frac{\partial \mathrm{w}_{e}}{\partial D_{n}} .
\end{aligned}
$$

Physically, Eq. (2.51) represents the energy released by loss of stiffness of the RVE where damage occurs. Also, the thermodynamic variables $Y_{s}$ and $Y_{n}$ are by definition the associated variables of the internal state variables (i.e. damage tensor) governing the phenomenon of damage (LEMAITRE, 2012). Moreover, for plane stress states and in material coordinate system, these can be written in terms of elastic material properties as,

$$
\begin{gathered}
Y_{s}=\frac{\sigma_{12}^{2}}{2 G_{12}\left(1-D_{s}\right)^{2}}, \\
Y_{n}=\frac{\sigma_{22}^{2}}{2 E_{22}\left(1-D_{n}\right)^{2}},
\end{gathered}
$$

which are the associated variables needed to model damage kinetic evolution laws (LEMAITRE, 2012). Yet, notice in Eq. (2.52) the non-zero $\sigma_{12}$ shear stress, indicating that it is not written in principal directions. Also, the damage dissipation $\mathcal{D}$ is simply,

$$
\mathcal{D}=Y_{s} \frac{\partial D_{s}}{\partial t}+Y_{n} \frac{\partial D_{n}}{\partial t}
$$

Considering that the principal directions are time independent and remain constant, damage will possess these same directions. Hence, the effective stresses in the principal directions in a three-dimensional (3D) coordinate system are given by,

$$
\hat{\sigma}=\left[\begin{array}{ccc}
\frac{\sigma_{11}}{1-D_{1}} & 0 & 0 \\
0 & \frac{\sigma_{22}}{1-D_{2}} & 0 \\
0 & 0 & \frac{\sigma_{33}}{1-D_{3}}
\end{array}\right]
$$

where $D_{1}, D_{2}$ and $D_{3}$ are the damage variables in principal directions for $3 \mathrm{D}$ stress states. Notice that in contrast with Eq. (2.52) for the thermodynamic forces in material coordinate system and plane stress state, Eq. (2.54) is written for a general stress state in principal directions, which can be highlighted by the absence of shear stresses in the effective stress tensor $\hat{\sigma}$. Thus, it is plain to observe that these directions are not the same, except for special cases. 


\subsubsection{Developed Material Models}

Ferreira's (FERREIRA et al., 2019) criteria is a phenomenological mesoscale two-dimensional (2D) CDM based material model. It is based on the Ribeiro's (RIBEIRO, 2013) model which, in its turn, is an extension of Ladevèze's (LADEVEZE; LEDANTEC, 1992) model. Thus, lamina homogenization is done to capture fiber and matrix damage modes without being capable of evaluating the fiber-matrix interface behavior. Also, it is applied for FRP composite materials with UD fiber reinforcement and have already proven to be a reliable model for quasi-static and dynamic problems. The main difference between Ferreira's and Ribeiro's material model is that the latest is a function of the ply orientation while the first is not.

The material model is capable of capturing tensile and compression failures in direction 1; tensile and compression failures in direction 2 and failure due to in-plane shear as well. In this sense, this is a intralaminar material model not being capable to capture the onset and progression of delamination, which is an interlaminar mode. Moreover, the first damage modes identification are made separately meaning that the onset of failure mechanisms are independent among them. This is justified by the great difference between fiber and matrix elasticity moduli. Nevertheless, the evolution of properties degradation is made considering that these damage modes interact between them, feature that can be verified through the modified constitutive matrix.

\section{Ply Behavior in Direction 1}

Experimental data collected by Soden et al. (SODEN; HINTON; KADDOUR, 2004) supports that for fiber failure of CFRP and GFRP (glass fiber-reinforced plastic) under tensile loading there is no interaction between stresses and only the longitudinal component needs to be considered. Provided by these results, many failure criteria models fiber failure using maximum strain or maximum stress criteria. For example, LaRC03 and Puck's failure criteria uses the maximum strain criterion. On the other hand, Pinho's (PINHO; IANNUCCI; ROBINSON, 2006a; PINHO; IANNUCCI; ROBINSON, 2006b) and Ferreira's failure models chooses for the maximum stress criteria usage. Besides this, all of these criteria agrees that FRP composites under tensile loading has a linear-elastic behavior and presents a brittle failure. The criterion is given by,

$$
\frac{\sigma_{11}}{X_{T}} \geq 1
$$

where $X_{T}$ is the longitudinal tensile strength. This criterion is also independent of fiber volume fraction and lamina Young's moduli. Besides this, it is associated to the internal damage variable $d_{1}$, which assumes a unitary value once failure is detected considering that the reinforcement is fully damaged (i.e. there is no gradual evolution of this parameter). To avoid computational issues of localization during simulations due to the abrupt change in value of $d_{1}$ damage variable, property degradation of this criterion is not done during 
each iteration procedure. Instead, it is made at the end of each time step of the FEM solution.

On the other hand, the compressive failure behavior is more difficult to model. Thus, in Ferreira's material model it assumes a linear-elastic stress-strain behavior until a threshold value is reach. This linear threshold is experimentally identified by conducting compression tests on $0^{\circ}$ specimens and is denoted by $X_{C_{0}}$. The criteria in the linear-elastic regime is written as,

$$
\frac{\left|\sigma_{11}\right|}{X_{C_{0}}} \geq 1
$$

Thus, after $\left|\sigma_{11}\right|$ reaches this threshold value, any increasing in compression load will result in a non-linear behavior that is simulated by a secant modulus strategy as given,

$$
E_{11}=\frac{X_{C_{0}}}{\left|\varepsilon_{11}\right|}\left[1-f\left(\varepsilon_{11}\right)\right]+f\left(\varepsilon_{11}\right) E_{11_{0}}
$$

in which $E_{11_{0}}$ is the initial Young's modulus in the longitudinal direction, $f\left(\varepsilon_{11}\right)$ is a strain function obtained from parameter fitting of $0^{\circ}$ coupons stress-strain curves, $\varepsilon_{11}$ is the strain component in the fiber direction and $E_{11}$ is the secant modulus. The parameter $f\left(\varepsilon_{11}\right)$ represents the linear equation that best fits the experimental data of the stress-strain curve. The linear-elastic stress threshold value $X_{C_{0}}$ is found by the intersection of the parallel curve possessing $0.2 \%$ strain value with the experimental curve (Figure 23a). Provided with the $X_{C_{0}}$ value, the strategy is to found the line that best fits the experimental curve and, consequently, the secant elasticity modulus. After that, the procedure is repeated successively until meeting good results as shown in Figure 23c.

Figure 23: Hypothetical compression stress-strain experimental curve (a), secant modulus (b) and successive secant modulus (c).

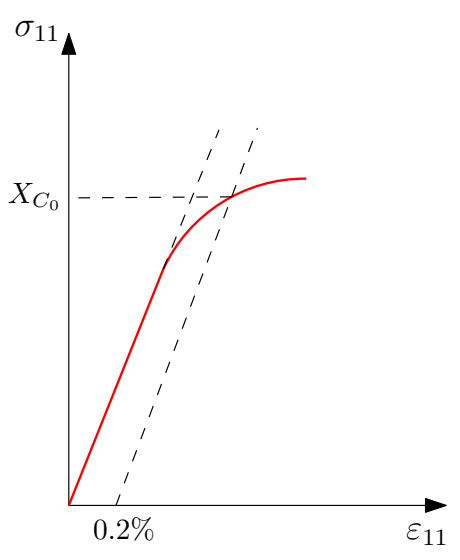

(a)

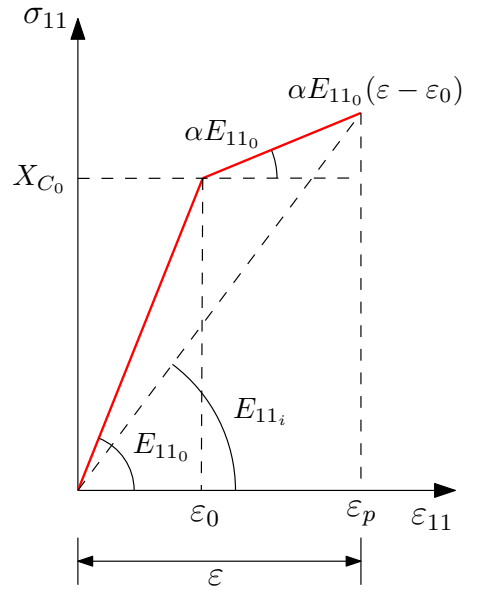

(b)

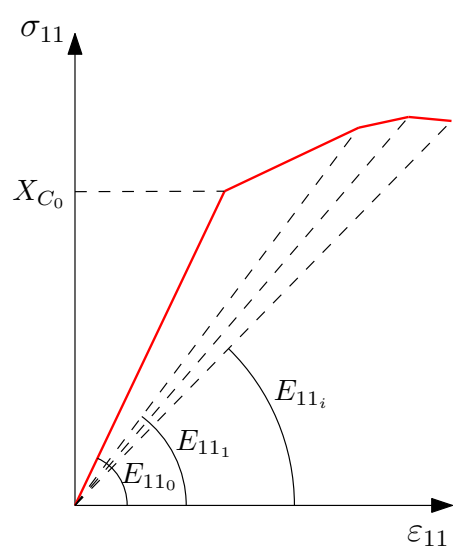

(c)

Source: adapted from (HERAKOVICH, 1998) by the author. 
Thus, the non-linear behavior is modelled by the secant modulus strategy. It follows that, from Figure 23b, the variables $\varepsilon, \varepsilon_{0}$ and $\varepsilon_{p}$ are the total strain, the strain at $X_{C_{0}}$ and plastic strain. Also, $\alpha E_{11_{0}}$ is the stiffness for the stress-strain curve above the elastic limit and $E_{11_{i}}$ is the secant modulus at the $i$-iteration where the parameter $\alpha \equiv f\left(\varepsilon_{11}\right)$ is considered for notation's sake.

Lastly, it can be well noticed the effect of loss of stiffness (i.e. damage) by the decay on the angular coefficient in each successive iteration.

\section{Ply Behavior in Direction 2 and due to In-Plane Shear}

Monotonic quasi-static tests conducted by Ribeiro (RIBEIRO, 2013) supports that the behavior in the direction 2 is non-linear under compression due to matrix inelastic deformations and damage process (PUCK; SCHÜRMANN, 2004; RIBEIRO, 2013). Also, in a plane stress state, damage process is governed by $\sigma_{22}$ (transverse loading) and $\tau_{12}$ (in-plane shear loading). Thus, Ribeiro proposed a damage threshold limit based on experimental fitting of tensile and compression test data of off-axis coupons. The threshold is given by,

$$
f=\sqrt{\sigma_{22}^{2}+\tau_{12}^{2}}-\left[\frac{2 S_{12_{y}}}{1+\left(\frac{\left|\sigma_{22}\right|}{Y_{C_{0}}}\right)^{3}}-S_{12_{y}}\right]
$$

where $Y_{C_{0}}$ and $S_{12 y}$ are the linear-elastic stress threshold values for compression and shear loadings, respectively. The strategy for obtaining these values is the same adopted for $X_{C_{0}}$. Another important thing to mention is that this model assumes that stress in fiber direction does not affect the damage state in the matrix. Ferreira (FERREIRA et al., 2019) uses this as base to propose another damage threshold limit which is a little more conservative than Ribeiro's for pure $\sigma_{22}$ and $\tau_{12}$ stress states. That is mainly due to the lack of off-axis experimental data for the studied material. The proposed modification is,

$$
f=1-\left[\frac{{\tau_{12}}^{2}}{{S_{12 y}}^{2}}+\frac{{\sigma_{22}}^{2}}{Y_{C_{0}}{ }^{2}}\right] .
$$

Figure 24 presents both Ribeiro's and Ferreira's damage threshold limit curves for the material used in their work. In it, the ply damage onset occurs when a pair of values is beyond the envelope limit. Also, it is depicted the off-axis $\left[70^{\circ}\right]_{6}$ and $\left[45^{\circ}\right]_{6}$, angle-ply $\left[ \pm 45^{\circ}\right]_{8 s}$ and $\left[90^{\circ}\right]_{8}$ data for the material used in the current work.

As noticed in Figure 24, there is also a curve-fitting failure envelope used in this work for the studied CFRP composite material. After performing the tests that are discussed in Chapter 3, it is realized that a different damage threshold limit should be proposed for the material used throughout this work. Then, the new failure envelope is 
Figure 24: Ply damage threshold limits.

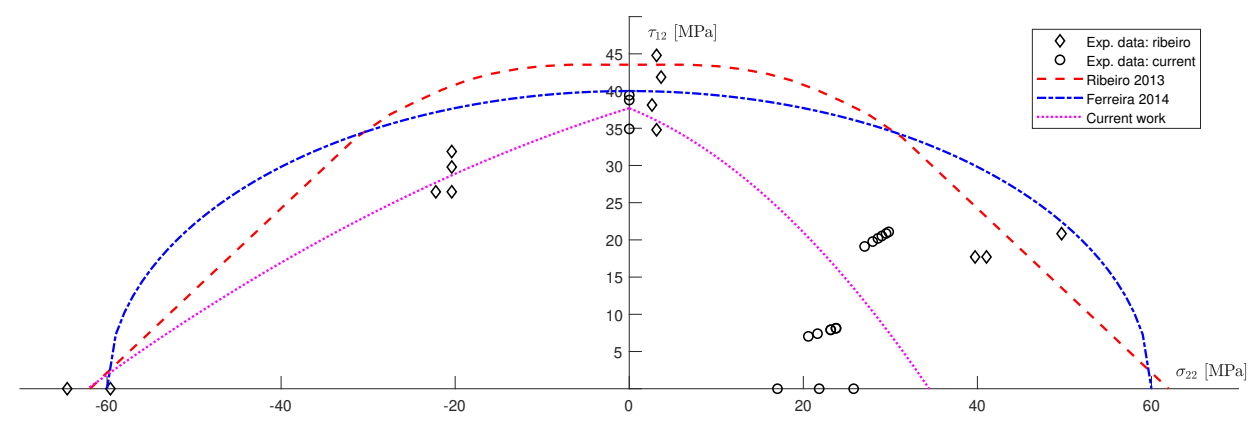

Source: reconstructed from (FERREIRA, 2014) by the author.

simply as follows,

$$
\begin{aligned}
& f_{+}=1-\left(\frac{-0.018 \sigma_{22}^{2}-0.474 \sigma_{22}+S_{12}}{\left|\tau_{12}\right|}\right), \text { for } \sigma_{22} \geq 0 \\
& f_{-}=1-\left(\frac{-0.004 \sigma_{22}^{2}+0.363 \sigma_{22}+S_{12}}{\left|\tau_{12}\right|}\right), \text { for } \sigma_{22}<0
\end{aligned}
$$

that, for both cases, it is valid that $f_{+,-}=0$ represents the threshold limit for damage onset. Although the present envelope does not possess intrinsic physical meaning it is better for the finite element investigations and it is simply empirically obtained. Furthermore, due to the lack of data for the negative portion of $\sigma_{22} \times \tau_{12}$ quadrant for the current material, the experimental data gathered by Ribeiro is used for constructing the $f_{-}$curve. It is probably not a big deal for the further computational investigations since these are held mainly at the positive quadrant of the envelope.

Analogously to the case of longitudinal loading, under compression there is a non-linear behavior starting after $Y_{C_{0}}$ value that can be simulated using the secant modulus strategy as well. It follows that,

$$
E_{22}=\frac{Y_{C_{0}}}{\varepsilon_{22}}\left[1-g\left(\varepsilon_{22}\right)\right]+g\left(\varepsilon_{22}\right) E_{22_{0}}
$$

in which $E_{22_{0}}$ is the initial Young's modulus in the transverse direction, $g\left(\varepsilon_{22}\right)$ is a strain function obtained from parameter fitting of $90^{\circ}$ coupons stress-strain curves (under compression), $\varepsilon_{22}$ is the strain component in the transverse direction and $E_{22}$ is the secant modulus. Like before, the parameter $g\left(\varepsilon_{22}\right)$ represents the linear equation that best fits the experimental data of the stress-strain curve. The procedure to determine $Y_{C_{0}}$ and $g\left(\varepsilon_{22}\right)$ is analogous to the fiber behavior case.

In order to model damage evolution in the matrix, two internal damage variables $d_{2}$, related to $\sigma_{22}$, and $d_{6}$, related to $\tau_{12}$, are introduced. Again, both of these parameters ranges from "0" for undamaged to "1" for fully damaged material. Based on CDM, the effective 
stress hypothesis relating these parameters to the lamina stress state (HERAKOVICH, 1998) is employed. Hence, considering that the effective stresses are applied on the damaged area and in the material coordinate system,

$$
\left\{\begin{array}{l}
\hat{\sigma}_{11} \\
\hat{\sigma}_{22} \\
\hat{\tau}_{12}
\end{array}\right\}=\left[\begin{array}{ccc}
\frac{1}{1-d_{1}} & 0 & 0 \\
0 & \frac{1}{1-d_{2}} & 0 \\
0 & 0 & \frac{1}{1-d_{6}}
\end{array}\right]\left\{\begin{array}{l}
\sigma_{11} \\
\sigma_{22} \\
\tau_{12}
\end{array}\right\} .
$$

Provided with effective stresses, it follows that the damaged strain energy density for the polymeric matrix is,

$$
E_{D}=\frac{1}{2}\left[\frac{\left\langle\sigma_{22}\right\rangle_{+}^{2}}{E_{22_{0}}\left(1-d_{2}\right)}+\frac{\left\langle\sigma_{22}\right\rangle_{-}^{2}}{E_{22_{0}}}+\frac{\left|\tau_{12}\right|^{2}}{G_{12_{0}}\left(1-d_{6}\right)}\right],
$$

where $\langle x\rangle$ is the Macauley brackets operator defined as,

$$
\begin{aligned}
& \langle x\rangle_{-}=\left\{\begin{array}{c}
-x, \text { if } x \leq 0, \\
0, \text { if } x>0 \\
+x, \text { if } x \geq 0 \\
0, \text { if } x<0
\end{array}\right.
\end{aligned}
$$

Moreover, Eq. (2.63) can be related to the thermodynamic forces, through the damage variables, for the ply (LADEVEZE; LEDANTEC, 1992). These are given as,

$$
\begin{aligned}
& Y_{2}=\frac{\partial E_{D}}{\partial d_{2}}=\frac{\left\langle\sigma_{22}\right\rangle_{+}^{2}}{2 E_{22_{0}}\left(1-d_{2}\right)^{2}}, \\
& Y_{6}=\frac{\partial E_{D}}{\partial d_{6}}=\frac{\tau_{12}^{2}}{2 G_{12_{0}}\left(1-d_{6}\right)^{2}} .
\end{aligned}
$$

Regarding to the evolution of the damage internal variables, once under transverse compression $d_{2}$ grows only when $\sigma_{22} \geq 0$. On the other hand, the parameter $d_{6}$ progresses independently of the sign of $\tau_{12}$. This is due to the presence of microvoids and microcracks in the ply. Thus, damage progression occurs when the lamina is under transverse compression and/or in-plane shear. In the first case, these defects can be closed and retard damage evolution (HERAKOVICH, 1998); this effect is named material self-healing. Since, damage onset in composite materials is identified through stiffness reduction, its propagation can be evaluated by means of quasi-static cyclic tests. Therefore, loss in stiffness can be identified in load-unload-reload cycles as shown in Figure 25.

Initially, for damage evaluation, it is assumed that the ply is in its pristine form $\left(d=0\right.$ for $\left.E_{0}\right)$. The degradation onset occurs when the non-linear behavior is reached. At this moment, the thermodynamic force $Y_{0}$ is calculated for $d=0$ and is given by,

$$
Y_{0}=\frac{\sigma_{0}^{2}}{2 E_{0}}
$$


Figure 25: Stiffness degradation due to damage and parameters calculation procedure.

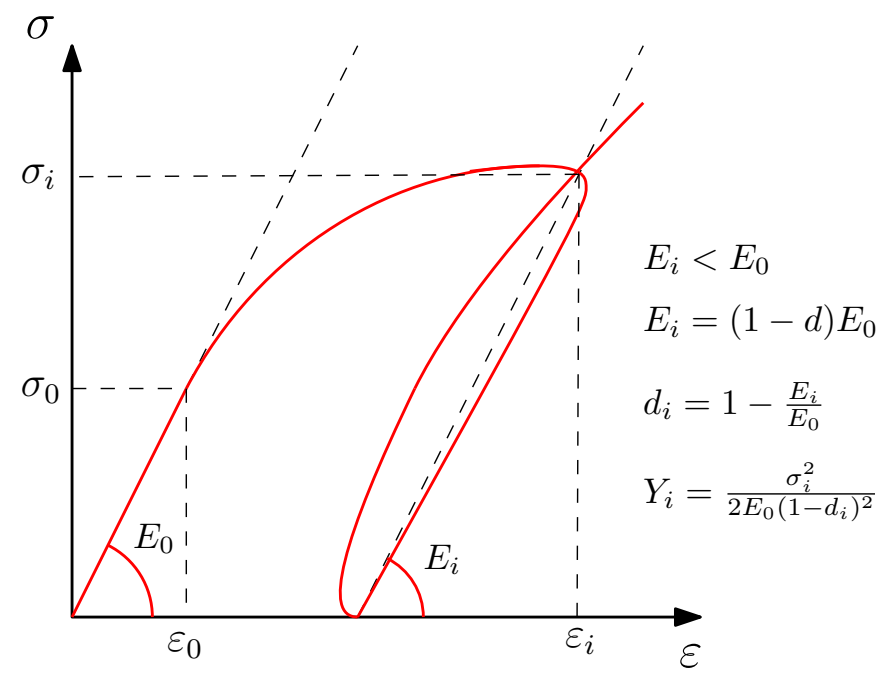

Source: adapted from (HERAKOVICH, 1998) by the author.

With increasing loading, the first cycle can present stiffness reduction with the damage parameter calculated as,

$$
d=1-\frac{E_{i}}{E_{0}}
$$

Furthermore, thermodynamic forces accounting for damage in a material point are calculated at the reloading stage as,

$$
Y_{i}=\frac{\sigma_{i}^{2}}{2 E_{0}\left(1-d_{i}\right)^{2}}
$$

and this procedure can be repeated as necessary. The maximum thermodynamic forces values associated to a loading history at any time $\tau$ until a current time $t$ are given by,

$$
\begin{aligned}
& \underline{Y}_{2}(t)=\max \left\{Y_{2}(\tau)\right\}, \\
& \underline{Y}_{6}(t)=\max \left\{Y_{6}(\tau)\right\},
\end{aligned}
$$

in which $\tau \leq t$. Another feature of the material model is the mutual influence between transverse and in-plane shear stresses in the damage process (LADEVEZE; LEDANTEC, 1992). This coupling varies from material to material and in Ferreira's model is given by a linear combination of the associated thermodynamic forces as stated by Ladevèze,

$$
\hat{Y}=Y_{6}+b Y_{2}
$$

where $b$ is the coupling coefficient and $\hat{Y}$ is the thermodynamic force related to the combined stress state. In the same fashion, it follows,

$$
\hat{\underline{Y}}(t)=\max \left\{Y_{6}(\tau)+b Y_{2}(\tau)\right\}
$$


is the maximum thermodynamic force experienced by the material, also with $\tau \leq t$. Notice that this coupling coefficient is a mathematical tool to assess the mutual influence of both transverse and in-plane shear stresses in the ply behavior. Thus, one should be cautious when obtaining it since, by construction, it must have a positive value but not necessarily varying between 0 and 1 as normally expected. If the last is desired, a normalization must be performed. This will be better explored in Chapter 3. Furthermore, there are other ways to couple these effects. For example, some authors (MIOT, 2009; MIOT; HOCHARD; LAHELLEC, 2010) use the following equivalent thermodynamic force:

$$
Y_{e q}=a Y_{2} d_{2}^{m}+b Y_{6} d_{6}^{n}
$$

in which $a, b, m$ and $n$ are the material parameters that characterizes the shear-transverse tension coupling and $Y_{e q}=\hat{Y}$ in this context. Also, other damage evolution laws are used in the aforementioned works.

Proceeding with the approach by Ladevèze, provided with the previous equations, damage evolution laws are given as,

$$
\begin{aligned}
& d_{6}=\frac{\left\langle\sqrt{\underline{\hat{Y}}}-\sqrt{Y_{0}}\right\rangle}{\sqrt{Y_{c}}}, \text { if } d_{6}<1 \text { and } Y_{2}<Y_{2}^{c} ; \text { otherwise } d_{6}=1, \\
& d_{2}=\frac{\left\langle\sqrt{\underline{\hat{Y}}}-\sqrt{Y_{0}^{\prime}}\right\rangle}{\sqrt{Y_{c}^{\prime}}}, \text { if } d_{2}<1 \text { and } Y_{2}<Y_{2}^{c} ; \text { otherwise } d_{2}=1,
\end{aligned}
$$

in which $Y_{2}^{c}$ is the tensile strength in the transverse direction. Also, the parameters $Y_{0}, Y_{0}^{\prime}, Y_{c}$ and $Y_{c}^{\prime}$ are determined in cyclic quasi-static tests. These damage evolution laws tendencies are represented in Figure 26.

Figure 26: Damage evolution due to shear (a) and transverse tension (b).

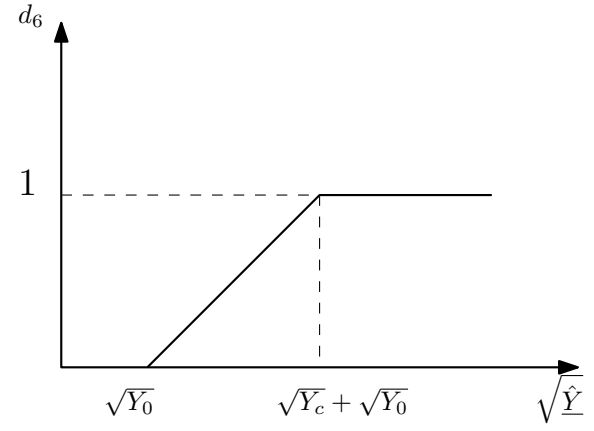

(a)

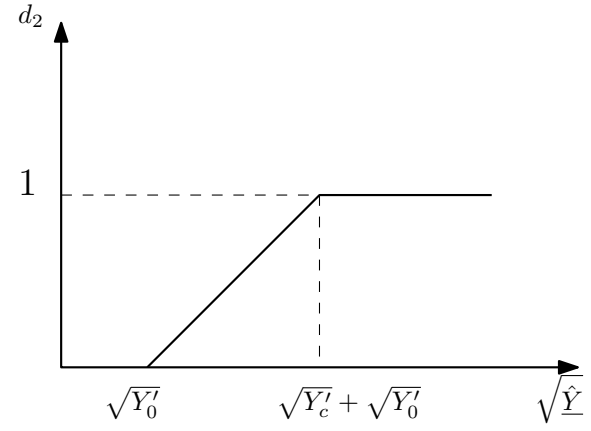

(b)

Source: adapted from (HERAKOVICH, 1998) by the author.

It is worth to mention that this material model accounts for the brittle behavior of the lamina by means of the strain energy density for the damaged matrix $E_{D}$. Hence, 
when $E_{D}$ is greater than a threshold value $E_{D C}$, experimentally obtained, the matrix is considered fully damaged (i.e. $d_{2}=d_{6}=1$ ). In short, Table 2 presents a summary of the adopted material model.

Table 2: Material model summary

\begin{tabular}{llc}
\hline Damage criterion & Ply damage mode & Degradation law \\
\hline$\frac{\sigma_{11}}{X_{T}} \geq 1$ & Tensile & $E_{11}=E_{11_{0}}\left(1-d_{1}\right)$ \\
& in direction 1 & $E_{11}=\frac{X_{C_{0}}}{\left|\varepsilon_{11}\right|}\left(1-f\left(\varepsilon_{11}\right)\right)+f\left(\varepsilon_{11}\right) E_{11_{0}}$ \\
$X_{C_{0}} \geq 1$ & Compression & $E_{22}=E_{22_{0}}\left(1-d_{2}\right)$ \\
$f \geq 0$ & in direction 1 & $E$ \\
& Tensile & \\
$f \geq 0$ & in direction 2 & $E_{22}=\frac{Y_{C_{0}}}{\varepsilon_{22}}\left(1-g\left(\varepsilon_{22}\right)\right)+g\left(\varepsilon_{22}\right) E_{22_{0}}$ \\
$f \geq 0$ & Compression & $G_{12}=G_{12_{0}}\left(1-d_{6}\right)$ \\
\hline
\end{tabular}

Finally, it follows that the modified constitutive matrix is given by (MATZENMILLER; LUBLINER; TAYLOR, 1995),

$$
D=\frac{1}{K}\left[\begin{array}{ccc}
\left(1-d_{1}\right) E_{11} & \left(1-d_{1}\right)\left(1-d_{2}\right) \nu_{21} E_{22} & 0 \\
\left(1-d_{1}\right)\left(1-d_{2}\right) \nu_{12} E_{11} & \left(1-d_{2}\right) E_{22} & 0 \\
0 & 0 & K\left(1-d_{6}\right) G_{12}
\end{array}\right]
$$

where $K=1-\left(1-d_{1}\right)\left(1-d_{2}\right) \nu_{12} \nu_{21}$. Moreover, in order to avoid the material self-healing, damage internal variables assumes always the maximum calculated values along the load history analyses.

\subsection{Impact Analyses for Composite Materials}

\subsubsection{Experimental Approach}

Currently, there are many possible experimental approaches across the literature to perform an impact test that are well described by present standards. In this context, some procedures are more suited for low-velocity, high-velocity and ballistic impacts than others. For example, the split-Hopkinson bar testing (SHBT) is recommended for ballistic tests simulating the impact between a gun projectile and a target. Thus, this type of testing is within the destructive approaches. In the same category there is the well-known charpy pendulum system used to measure the resilience of a material. On the other hand, for low-velocity impacts performed on composite materials there are the non-destructive drop-weight tests described by the ASTM D5628 and D7136 standards. The first one recommend the test to be realized with a circular coupon fixed by bolts with some specified load. The last one, also known as Boeing standard, specify a rectangular specimen to be impacted by a hemispherical aluminum indenter. The specimen is fixed with four toggle 
clamps in a inertial base (i.e. the mass of this base is much higher than the mass of the specimen/impactor and can be considered as a rigid body being ignored in the analysis) as represented in Figure 27.

Figure 27: Specimen fixture (a) and rigid base (b) described in the ASTM D7136 standard.

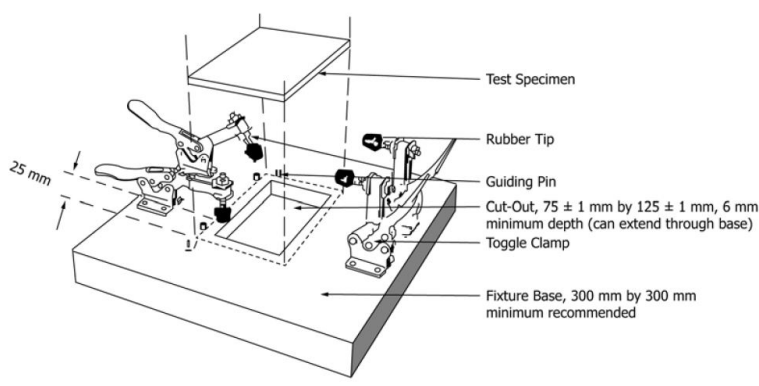

(a)

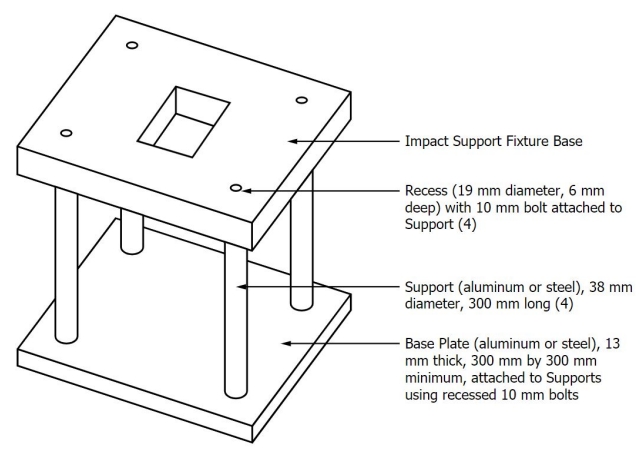

(b)

Source: American Society for Testing and Materials.

Obviously, this fixture system is less conservative than the one depicted in the D5628 standard (Figure 28) and it makes the boundary conditions pretty non-straightforward. This happens because the ASTM D7136 standard was made with the objective of performing residual strength assessment through compression-after-impact (CAI) testing.

Figure 28: Specimen fixture of the ASTM D5628 standard.

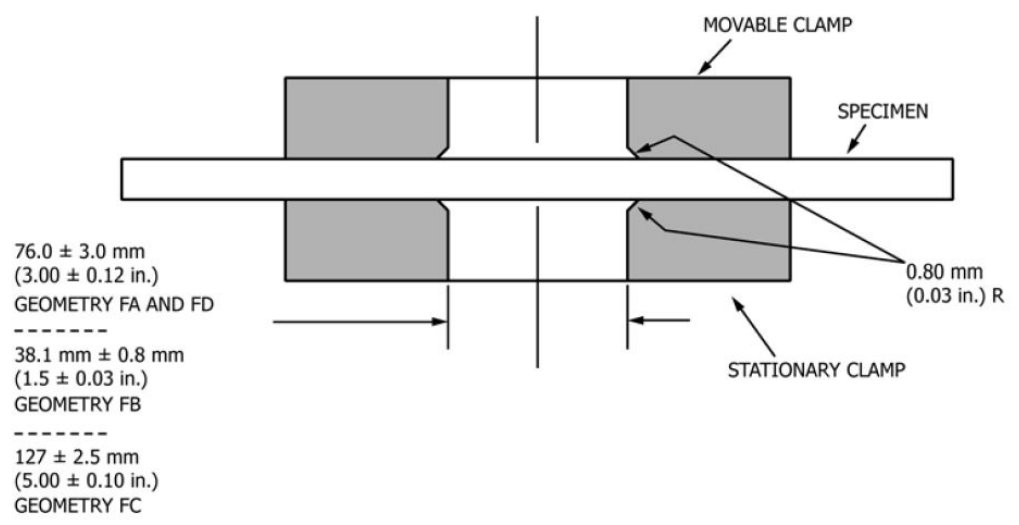

Source: American Society for Testing and Materials.

For computational purposes, it is highly recommended that a extensive boundary condition (BC) study is performed although in LVI the influence of the BC can sometimes be neglected. Thus, some previous simulations with well-behaved materials (such as aluminum) needs to be made to calibrate the numerical model with the experimental apparatus. Moreover, the drop-weight test is very flexible with respect to the kinetic energy level. It allows control of the height and mass of the impactor with some regards to energy 
losses through friction of the support with the rails. Some results of force and energy histories are exemplified in Figure 29.

Figure 29: Force (a) and energy (b) histories examples for several LVI energy levels.

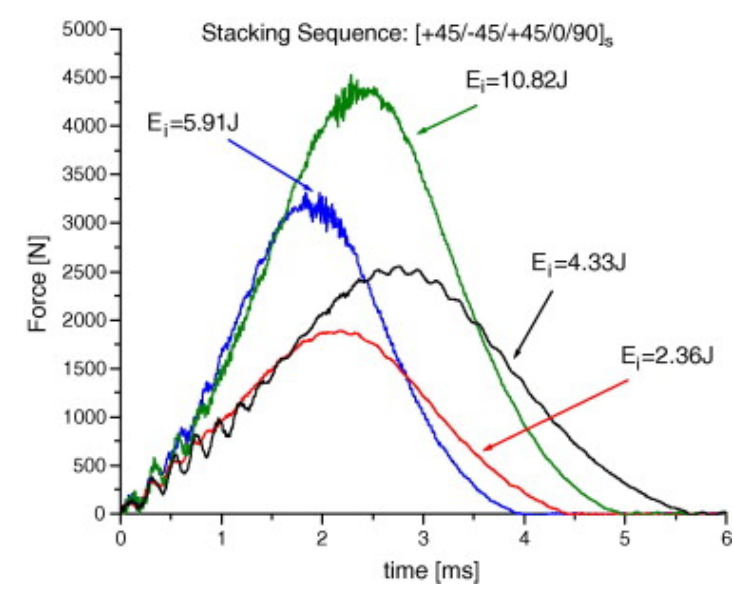

(a)

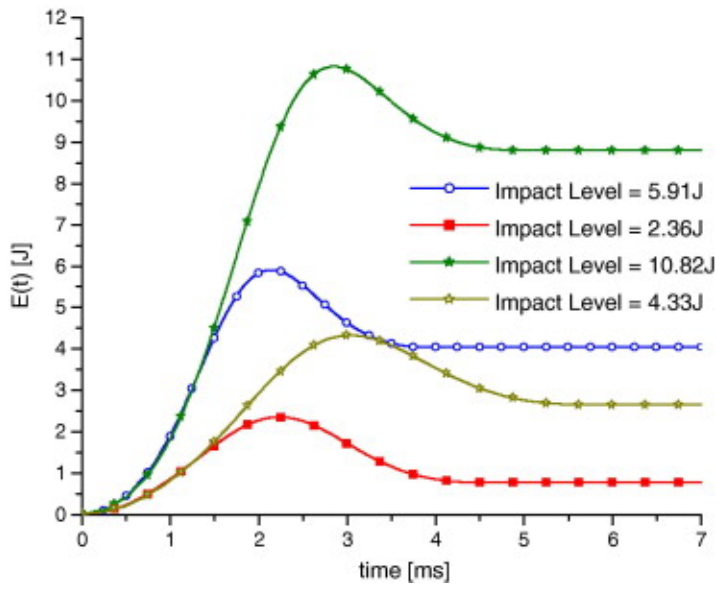

(b)

Source: after Tita, Carvalho and Vandepitte (TITA; CARVALHO; VANDEPITTE, 2008).

Firstly, it is worth mention that the displacement history (not shown) is very similar with the force one. Next, it is visible that the peak force increases with higher energy levels as expected. Also, in the work by Tita (TITA; CARVALHO; VANDEPITTE, 2008) the impactor mass for the impact energies of 2.36 and $5.91 \mathrm{~J}$ is $1.205 \mathrm{~kg}$ and the impactor mass for the energies of 4.33 and $10.82 \mathrm{~J}$ is $2.205 \mathrm{~kg}$. Thus, it can be noticed in Figure 29a that the impact duration is directly related to the impactor mass and not to the impact energy $\left(E_{i}\right)$ level itself. The initial region of the force history is dominated by high frequency inertial oscillations due to the contact of the impactor with the target. Posterior oscillations indicate the occurrence of damage and/or failure and, at very low energy levels, it can be noticed a quasi-static behavior. Related to the energy history, it can be seen in Figure $29 \mathrm{~b}$ that the absorbed energy $\left(E_{a}\right)$ grows for higher impact energy levels being proportional to the quantity of activated failure mechanisms and the size of the damaged area in the impacted specimen. The absorbed energy is the energy dissipated by the laminate on the form of damage/failure and permanent strains. Also, the other portion of the impact energy that is stored in the specimen by means of elastic waves and returned to the impactor by vibrations is the elastic energy $\left(E_{e}\right)$ as depicted in Figure 30.

The impact energy is given by

$$
E_{i}=\frac{m v_{0}^{2}}{2}
$$

with $m$ and $v_{0}$ the impactor mass and initial velocity (at the contact imminence), respectively. Neglecting energy losses by friction, the impact energy is equal to the 
Figure 30: Energy history scheme: absorbed and elastic portions.

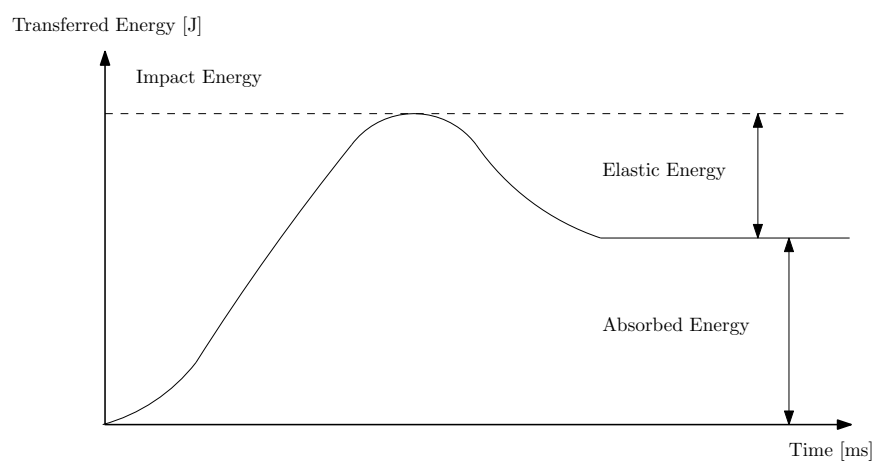

Source: adapted from (TITA, 2003) by the author.

gravitational potential energy

$$
E_{i}=m g h
$$

where $h$ is the impactor height and $g$ is the local accelaration of gravity. Then, the kinetic energy $\left(\mathcal{K} \mathcal{E}_{i}\right)$ transferred from the impactor to the composite plate is

$$
\mathcal{K E}_{i}(t)=E_{i}-\frac{m v_{i}^{2}(t)}{2}=\frac{m}{2}\left[v_{0}^{2}-v_{i}^{2}(t)\right]
$$

where $v_{i}$ is the impactor velocity through time. The last is calculated as

$$
v_{i}(t)=v_{0}-\frac{1}{m} \int_{0}^{t} F_{\exp }(t) d t
$$

in which $F_{\exp }$ is the experimental measured force through time. Of course, by Newton's Second Law it follows

$$
F_{\exp }(t)=\frac{d}{d t}\left[m \frac{d \delta_{i}(t)}{d t}\right]
$$

where $\delta_{i}$ is the experimental displacement, measured by the LDS equipment. Still, accordingly to Figure 30 it is true that

$$
\mathcal{K} \mathcal{E}_{i}(t)=E_{e}+E_{a}
$$

the $i$ index on Equations (2.75) through (2.80) are simply to count the collected samples during the drop-tests. Also, with the force and displacement histories, one can plot the displacement-force curve of the impact event as shown in Figure 31.

In this, as done by Tita (TITA; CARVALHO; VANDEPITTE, 2008), it can be noticed the existence of indentation test curves. As discussed in Tita's thesis (TITA, 2003), in some cases where the quasi-static behavior dominates the laminate response, the impact test can be well described by an indentation test. Thus, the drop-test is made to capture the peak force of the indentation one. Besides that, in impacts with several failure mechanisms this plot allows to capture the permanent displacement of the specimen. 
Figure 31: Displacement against force plot.

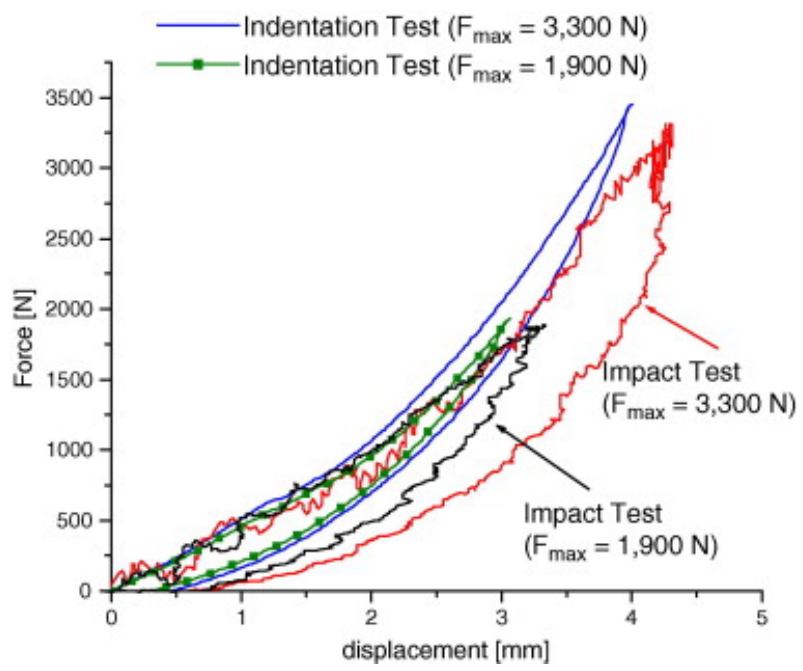

Source: after Tita, Carvalho and Vandepitte (TITA; CARVALHO; VANDEPITTE, 2008).

Another important analysis conducted by Tita (TITA; CARVALHO; VANDEPITTE, 2008 ) is the quantitative evaluation of the absorbed energy against the impact energy level (Figure 32).

Figure 32: Absorbed energy x Impact energy.

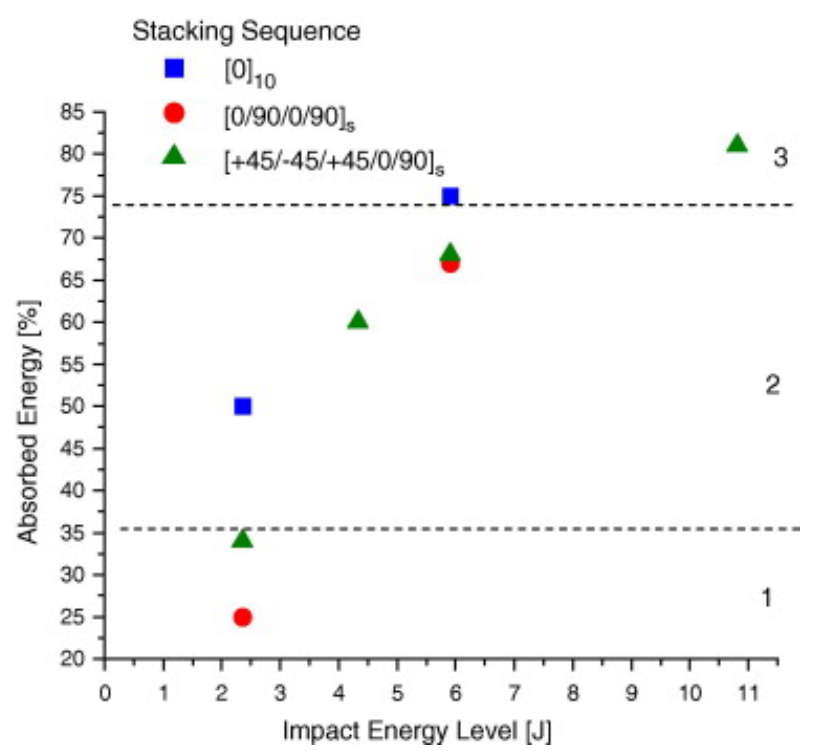

Source: after Tita, Carvalho and Vandepitte (TITA; CARVALHO; VANDEPITTE, 2008).

Notice that there are three regions defined. The first one covers the quasi-static behavior of specimens with very low absorbed energy fraction (under 35\%) and no activation of important failure mechanisms. Region 2 is characterized by matrix cracks and delaminations with an intermediate level of energy absorption between $35 \%$ and $75 \%$. 
Finally, in the last region the specimens presents high absorbed energy (over 75\%) during impact with fiber breakage failure existence.

\subsubsection{Computational Approach}

It is natural to approach a problem that it is poorly investigated by an experimental procedure. The issue in this strategy is when a massive number of tests are needed to qualify a single structure. Since composite materials are often used with the purpose of a lightweight design, it is not recommended to adopt a completely experimental approach to impact analysis in such structures. Thus, usage of numerical models to improve design conservative draw-backs are attractive and currently applied. In this sense, every impact model has in common a major aim that is being capable of predicting damage. With this purpose, the literature presents several types of modeling techniques that goes from one degree of freedom (DOF) analytical/empirical relations to micro-scale finite element models. Thus, it is straightforward to compare the existing models by means of its capability of prediction of damage and the effort (i.e. expend analysis time) it takes to finish the analysis. In this context, Bogenfeld et. al. (BOGENFELD; KREIKEMEIER; WILLE, 2018) come with a review/benchmark study of the computational techniques currently used to model impact mechanics. In their work, focus is put on stacked layer models with solid or shell elements with a cohesive zone method (CZM) approach for analysis of delamination. Figure 33 shows an overview of the methods considered.

Figure 33: Overview of impact analysis methods.

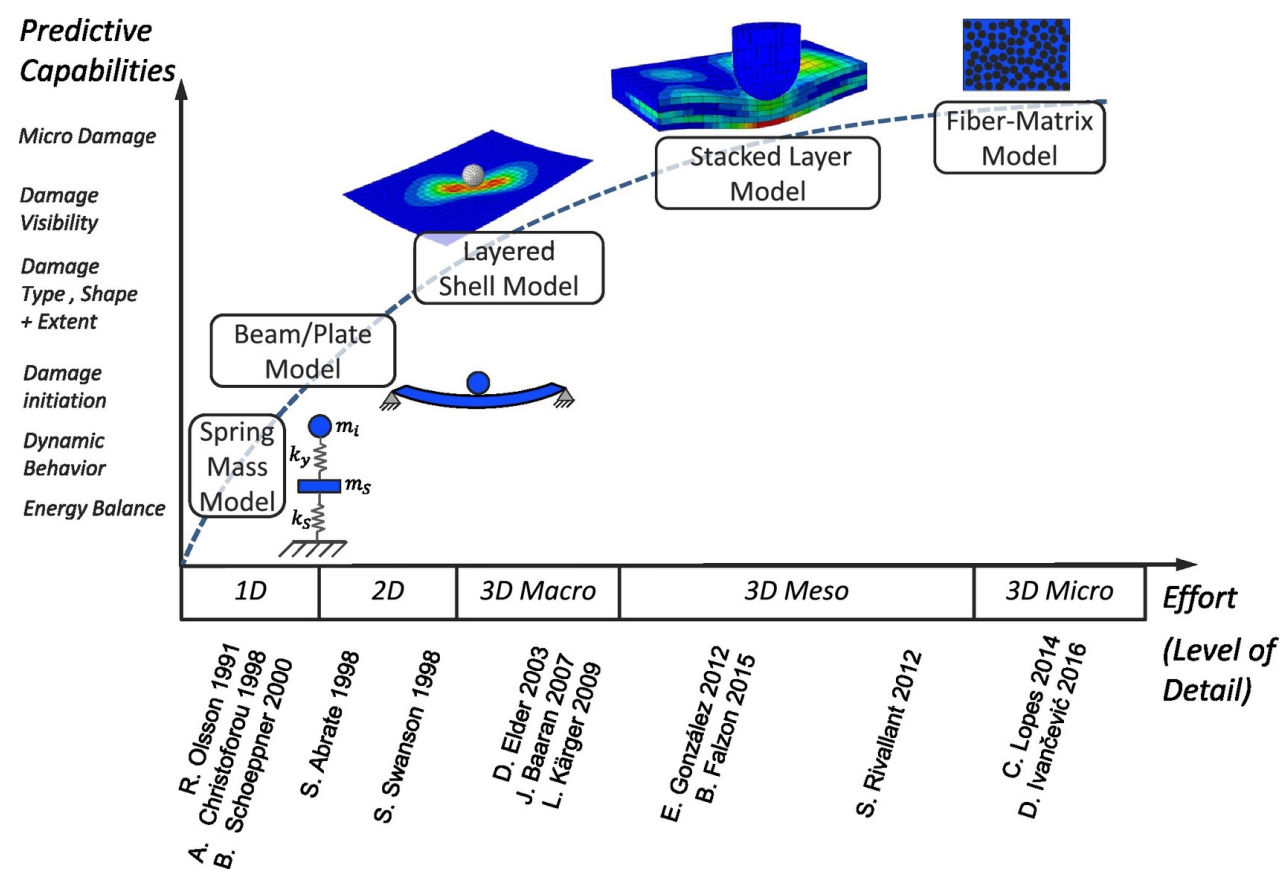

Source: after Bogenfeld, Kreikemeier and Wille (BOGENFELD; KREIKEMEIER; WILLE, 2018). 
Regarding to the cohesive zone method it is worth mentioning that this approach was firstly developed to model the adhesive fail of two adherents united in the context of a bonded joint. This problem is naturally distinct from the fail driven by delamination of two composite plies that are "united" after passing through a curing process. Thus, the present author believes that the CZM does not provide a physically-based approach to model this kind of mechanism. Also, for its correct usage it is necessary the previous knowledge of the location were delaminations will occur which can be very difficult to kwnow. Moreover, the cohesive zone method demands high computational power to model delamination in composites since it requires the insertion of more plies in the laminate where the cohesive law will govern the problem, e.g. in a laminate with $N$ laminae there is necessary to insert $N-1$ cohesive plies (for $N \geq 2$ ).

It can be well noticed that high-fidelity models with greater level of detail takes more effort to evaluate the damage onset and its propagation. In this sense, the techniques began with analytical one-dimensional strategies firstly introduced by Abrate (ABRATE, 1991) in the early 90's with a spring-mass model and followed by Olsson (OLSSON, 1992) deriving the first impact model based on Kirchhoff's plate equation. Posteriorly, a lot of analytical methods have been developed and improved by many researchers. Some shared characteristics between these models can be noticed with respect to its capabilities that are:

- Damage onset and its quantitative dimensions prediction;

- Treatment of the impact mechanics as a multi-body system (e.g. springs connected to one or more masses);

- Captures the elastic impact response taking into account the structure displacement and its local surface indentation;

- Prediction of impact duration, maximum contact force and impactor displacement.

It is worth noting that these capabilities of the analytical approaches are not so precise since these models are based on simplified hypothesis that make the problem solvable in reasonable manners. Moreover, there are even models that accounts for Damage Threshold Loads (DTL) to predict the contact forces that makes a delamination grow.

The next level of analysis is the computational one and it is based on numerical methods. Among these strategies adopted to simulate impact, the most common one is based on the Finite Element Method (FEM) and depending on the laminate's type of finite element abstraction scale can the problem lies on the macro-, meso- or micro-scales with growing fidelity and duration of analysis, respectively. The first level of abstraction is at the macro-scale and the FEM models considers a layered-shell approach to capture 
impact kinematics like in a plate model and evaluate failure conditions on the ply-level accounting for its corresponding stiffness degradation. Improvements can be made by stacking sublaminates together with an interface model between the plies (JOHNSON; PICKETT; ROZYCKI, 2001) which is the basis for the meso-scale models that are the next level of abstraction. In these, each ply is modeled with at least one layer of finite elements. The intralaminar and interlaminar damage models can be based on many theories such as the criteria developed by Hashin (HASHIN; ROTEM, 1973), Puck (PUCK; SCHÜRMANN, 2004) and many others. Here, attention is given to Continuum Damage Mechanics (CDM) for intralaminar damage models. Another important thing to mention about the meso-scale modeling techniques are that all of them works with homogenized failure conditions. Thus, without an experimental investigation it is only possible to say how the homogenized ply fails under tensile, compression or shear loadings in the directions 1, 2 or 1-2 plane.

Finally, the higher-fidelity level of analysis is at the micro-scale optics. Obviously, it is the methodology with greatest simulation effort in consequence. As a consequence, it gives detailed results with great prediction capabilities. Within these, the major improvement is the ability of distinguish between matrix, fiber or fiber-matrix interface damage and failure modes. The first impact model at this level of abstraction is the one by Lopes et. al. (LOPES et al., 2014). Such micro-scale models use as strategy the coupling of micromechanical models with a Reference Volume Element (RVE) in a multiscale approach and especially for multiaxial loading this procedure is superior to homogenized meso-scale models (BOGENFELD; KREIKEMEIER; WILLE, 2018).

The present work focus on the meso-scale level of analysis. So, the composite lamina is considered a homogeneous media although a fiber-reinforced polymer is essentially heterogeneous and, therefore, no distinction between fiber, matrix or fiber-matrix interface damage and failure modes are made. In this scenario, this text addresses intralaminar damage and failure, with this being fundamental to evaluate the residual strength considering post-impact shear loads. Of course, it is imperative to model delamination phenomenon when dealing with composite structures to fully develop an analysis procedure to treat with these. Nevertheless, this is not an easy task and involves many challenges essentially for impact events. According to Lin and Waas (LIN; WAAS, 2019) there are two major damage modes that influence delamination onset and growth that are matrix splitting and fiber kinking. Also, it is dependent on the plies position in the stacking sequence of the laminate. For the top most plies, both fiber kinking and matrix splitting plays important roles on delamination occurrence. By the other hand, at the middle plies only matrix splitting of the upper and lower plies is relevant. So, the key factor for any material model to assess delamination is to consider the interaction between these damage mechanisms and model how them leads to delamination phenomenon. Yet, one needs to capture its morphology that, for example, in a $[45 / 0 /-45 / 90]_{3 s}$ laminate studied by Lin and Waas (LIN; WAAS, 2019) during LEI it is observed a fan-shaped delamination pattern 
as shown in Figure 34 which is obtained by the micro-CT technique.

Figure 34: Fan-shaped delamination morphology.
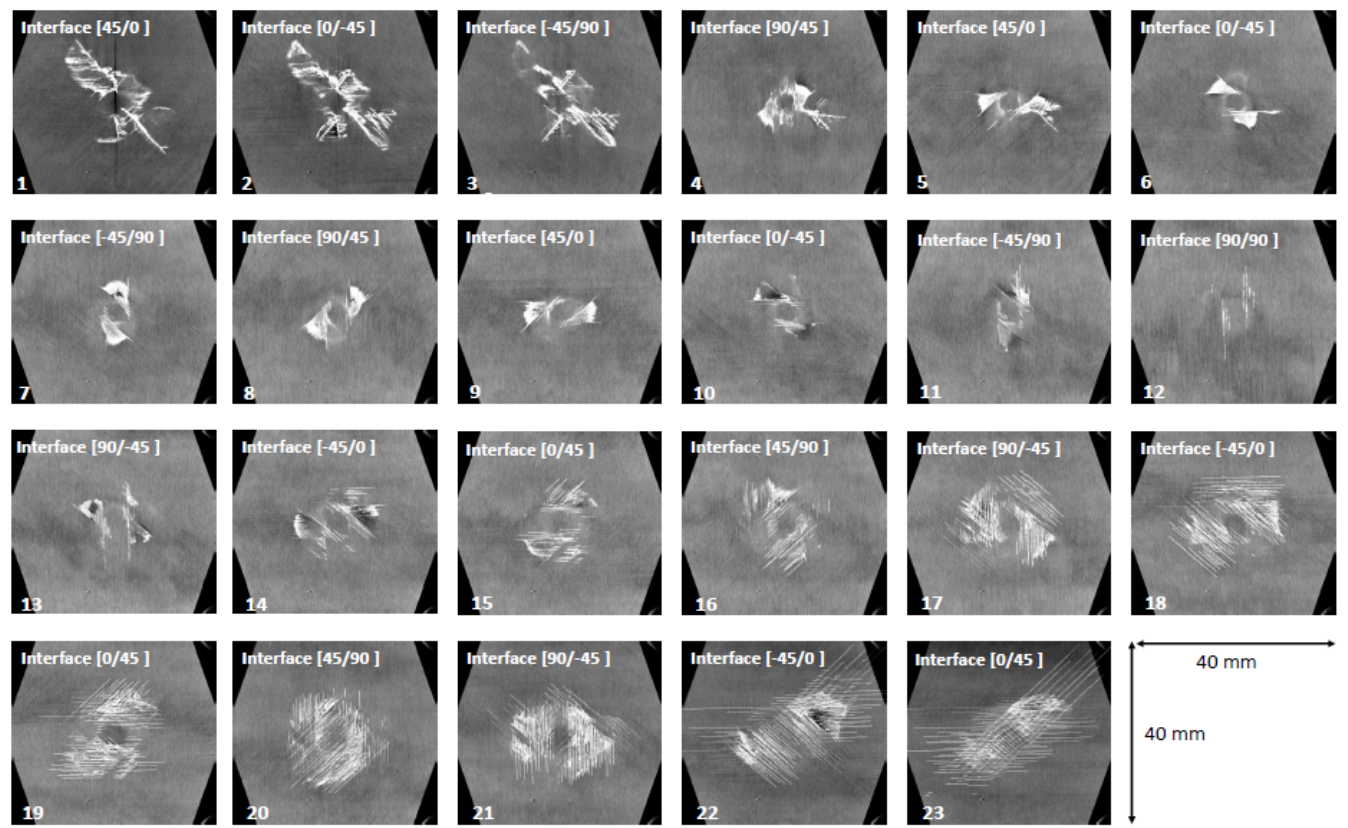

$40 \mathrm{~mm}$

$40 \mathrm{~mm}$

Source: after Lin and Waas (LIN; WAAS, 2019).

Therefore, this work deals, as aforementioned, only with intralaminar phenomena and, consequently, with laminates with low through-the-thickness stiffness gradients culminating in damage due to matrix cracking when under LEI, for example. In this sense, future efforts must be directed to predict delamination onset and growth. However, in this case, firstly it will be needed a consistent prediction of the out-of-plane stresses $\left(\sigma_{33}\right.$, $\tau_{13}$ and $\left.\tau_{23}\right)$. Thus, a 3D analysis needs to be employed using solid finite elements or a quasi-3D strategy using finite elements based in Unified Formulations (CALIRI JUNIOR; TITA; FERREIRA, 2016; CALIRI JUNIOR; FERREIRA; TITA, 2016; CALIRI JUNIOR; FERREIRA; TITA, 2017; CALIRI JUNIOR, 2017; RIBEIRO et al., 2018; FERREIRA et al., 2020).

Still, in this context, Lin and Waas (LIN; WAAS, 2019) summarizes some of the numerical models existing in the literature to account for both intra-inter ply mechanisms as shown in Table 3.

In it, the cohesive contact of Bouvet et al. (BOUVET; RIVALLANT; BARRAU, 2012) is provided by the in-built definition of ABAQUS; COH3D8 used by Falzon et al. (DONADON et al., 2008), Soutis et al. (SHI; SWAIT; SOUTIS, 2012; SHI; PINNA; SOUTIS, 2014) and Gonzalez et al. (GONZÁLEZ et al., 2012) stands for the 3D cohesive element with 8 nodes of ABAQUS; the DCZM is simply the discrete cohesive zone method approach employed by Thorsson et al. (THORSSON et al., 2018; THORSSON, 2017; THORSSON; WAAS; RASSAIAN, 2018b; THORSSON; WAAS; RASSAIAN, 2018a). 
Table 3: Summary of numerical models in literature (after Lin (LIN; WAAS, 2019))

\begin{tabular}{llccccc}
\hline & & Bouvet et al. & Falzon et al. & Soutis et al. & Gonzalez et al. & Thorsson et al. \\
\hline \multirow{3}{*}{ Fiber failure } & Tensile failure & Yes & Yes & Yes & Yes & Yes \\
& Compressive failure & Yes & Yes & Yes & Yes & Yes \\
& Fiber Crushing & Yes & Yes & Yes & No & No \\
\hline \multirow{3}{*}{ Matrix damage } & Nonlinear shear & No & Yes & Yes & No & Yes \\
& Inelastic strain & Yes & Yes & Yes & No & No \\
& Matrix crushing & No & No & No & No & Yes \\
\hline Delamination & & Cohesive contact & COH3D8 & COH3D8 & COH3D8 & DCZM \\
\hline
\end{tabular}

As discussed, one of the most popular approaches to deal with impact problems is within the framework of the Finite Element Method. Its objective is to transform the governing equations of one problem that usually posses second order derivatives into equations possessing first order derivatives to ensure symmetry on the stiffness matrix. The set of governing equations along with the boundary conditions is named Strong Form and its integral form is called Weak Form (JACOB; TED, 2007). The FEM is used to solve the weak form due to weaker continuity requirements. Thus, the solutions of the weak form need not to be as smooth as the solutions of the strong form and, combined with some well-selected approximation functions, the discrete finite element equations can be obtained. Of course, inside the set of all functions that are admissible trial solutions of the problem, the selected ones have to assure the capability to approximate a given smooth function with arbitrary accuracy; this is called completeness. One way to obtain, the transformation from the strong to the weak form is made through Green's Formula (REDDY, 1993).

In implicit FEM solutions of quasi-static equilibrium problems the momentum balance is solved neglecting inertia terms. For structural problems, the fundamental variable to be known is the displacement field that, in each time step, satisfies equilibrium and the essential, or Dirichlet, boundary conditions. This displacement field is found by solving the weak form (already discretized) of momentum balance or equilibrium equation. Therefore, equality between external and internal forces is required and, it follows that,

$$
\mathbf{f}^{\text {int }}=\mathbf{f}^{\text {ext }}
$$

where $\mathbf{f}^{\text {ext }}$ is the external forces vector and $\mathbf{f}^{\text {int }}$ is the internal forces vector. The last is a function of the unknown displacement field and, notice, Eq. (2.81) is actually a set of equations.

Dividing the problem domain into a mesh of finite elements, nodes are defined as the set of points where these elements intersects. Thus, reduction of order of the problem given in Eq. (2.81) is achieved by this procedure where the displacement field is also discretized providing finite equally sized sets of degrees of freedom and shape functions. These functions are defined in such a manner that the nodal displacements are 
interpreted as the DOFs of the problem. It follows that the element-wise representation of the displacement field $\mathbf{u}^{T}=\left\{u_{x}, u_{y}, u_{z}\right\}$ is given as,

$$
\mathbf{u}(\mathbf{x})=\mathbf{N}(\mathbf{x}) \mathbf{a}
$$

in which $\mathbf{N}$ and $\mathbf{a}$ are the shape function matrix and nodal displacement vector, respectively. Those are given by,

$$
\begin{aligned}
\mathbf{N} & =\left[\begin{array}{ccccccc}
N_{1} & 0 & 0 & \cdots & N_{n} & 0 & 0 \\
0 & N_{1} & 0 & \cdots & 0 & N_{n} & 0 \\
0 & 0 & N_{1} & \cdots & 0 & 0 & N_{n}
\end{array}\right], \\
\mathbf{a}^{T} & =\left\{\begin{array}{lllllll}
a_{1 x} & a_{1 y} & a_{1 z} & \cdots & a_{n x} & a_{n y} & a_{n z}
\end{array}\right\},
\end{aligned}
$$

where $n$ is the number of nodes in one element, $N_{i}$ are the shape functions defined over the element domain and $a_{i j}$ is the displacement of the $i$-th node in the $j$-direction (MEER, 2012). The strain field can be defined as,

$$
\varepsilon=\mathbf{B}(\mathbf{x}) \mathbf{a}
$$

with $\mathbf{B}(\mathbf{x})=\mathbf{L N}(\mathbf{x})$ being the strain nodal displacement matrix. Also, with the partial derivative matrix operator $\mathbf{L}$ defined as,

$$
\mathbf{L}^{T}=\left[\begin{array}{cccccc}
\frac{\partial}{\partial x} & 0 & 0 & 0 & \frac{\partial}{\partial z} & \frac{\partial}{\partial y} \\
0 & \frac{\partial}{\partial y} & 0 & \frac{\partial}{\partial z} & 0 & \frac{\partial}{\partial x} \\
0 & 0 & \frac{\partial}{\partial z} & \frac{\partial}{\partial y} & \frac{\partial}{\partial x} & 0
\end{array}\right] .
$$

Provided with Eq. (2.84) and a constitutive equation relating stress with strain (i.e. describes the material behavior), the stress field can be obtained from the nodal displacements. Therefore, the internal forces vector is evaluated with the calculated stress field in a loop over the elements (MEER, 2012),

$$
\mathbf{f}^{\text {int }}=\sum_{e} \mathbf{M}_{e} \int_{\Omega_{e}} \mathbf{B}^{T} \sigma d \Omega,
$$

where $\mathbf{M}_{e}$ is the incidence matrix, $\Omega_{e}$ is the element domain and a Galerkin approximation methodology is employed. If the internal forces vector is non-linear, a iterative solution using the Newton-Raphson procedure is required. In this strategy, the solution vector is updated in each iteration $j$ as,

$$
\mathbf{a}_{j}=\mathbf{a}_{j-1}+\mathbf{K}_{j-1}^{-1}\left[\mathbf{f}^{\mathrm{ext}}-\mathbf{f}^{\mathrm{int}}\left(\mathbf{a}_{j-1}\right)\right],
$$

in which $\mathbf{K}_{j-1}$ is the global tangent matrix evaluated at $\mathbf{a}_{j-1}$ and defined as,

$$
\mathbf{K}_{j-1}=\left.\frac{\partial \mathbf{f}^{\text {int }}}{\partial \mathbf{a}}\right|_{\mathbf{a}=\mathbf{a}_{j-1}}
$$


This procedure is repeated until some tolerance is reached (i.e. desired accuracy) and, then, the next time step starts. Also, the global tangent matrix is evaluated in a loop over the elements (MEER, 2012) taking the integral form below.

$$
\mathbf{K}=\int_{\Omega} \mathbf{B}^{T} \mathbf{D B} d \Omega
$$

Here, $\Omega$ is the complete analysis domain (or global domain) and $\mathbf{D}$ is the material tangent matrix which is a linearization of the constitutive law and, so, it corresponds to the stiffness $C_{i j k l}$ tensor at the elastic regime, with the prior being defined as,

$$
\mathbf{D}=\frac{\partial \sigma}{\partial \varepsilon}
$$

The last is also called Jacobian matrix. Lastly, both Eqs. (2.86) and (2.89) are evaluated in loops over the integration points.

Nowadays, there are many FE softwares that are in the state-of-the-art of development. Consequently, implementation of in-house FE codes for impact analysis is in most cases unproductive. In this context, Abaqus is a well-known finite element software with some features that enables the implementation of user material models through coupling of UMAT (User MATerial) and VUMAT subroutines for implicit (ABAQUS Standard) and explicit (ABAQUS Explicit) simulations, respectively. Therefore, it enables the implementation of the constitutive behavior of a material that is not present in the standard material library of the software using FORTRAN language. Figure 35 shows the schematic flowchart of both UMAT and VUMAT subroutines.

As aforementioned, the main difference between an UMAT and a VUMAT is the adopted numerical solution strategy: implicit and explicit in time, respectively. The UMAT subroutine functioning is described as it follows. Firstly, for every integration point of the finite elements and at all time steps, ABAQUS calls the user subroutine loads all state variables of the material model and calculate the stress tensor and the compatible Jacobian matrix $(\partial \sigma / \partial \varepsilon)$ of the given strain state satisfying equilibrium conditions. With the Jacobian matrix in hand, the strain tensor at the current iteration is obtained with the one previously calculated and the strain increment $\left({ }^{i} \varepsilon={ }^{i-1} \varepsilon+\Delta \varepsilon\right)$. In the next step, provided with the stress tensor prediction $\left({ }^{P} \sigma={ }^{i-1} C:{ }^{i} \varepsilon\right)$, the subroutine verifies the failure criterion condition. If damage or failure is identified then there is an update of the stiffness tensor by means of its degraded properties (i.e. recalculation of stress tensor and Jacobian matrix) and if no damage is identified then there is no update in the properties of the stiffness tensor and it will be the same as the one of the latest time steps. Also, if failure is detected in one step, it will be propagated to the next ones to avoid material self-healing. Finally, the effective stress tensor is evaluated and there is an update of the state variables; control of the analysis returns to ABAQUS Standard ending the loop. If there is convergence this means that the $R$-value (i.e. residue value) is smaller than the 
Figure 35: UMAT subroutine (a) and VUMAT subroutine schemes (b).

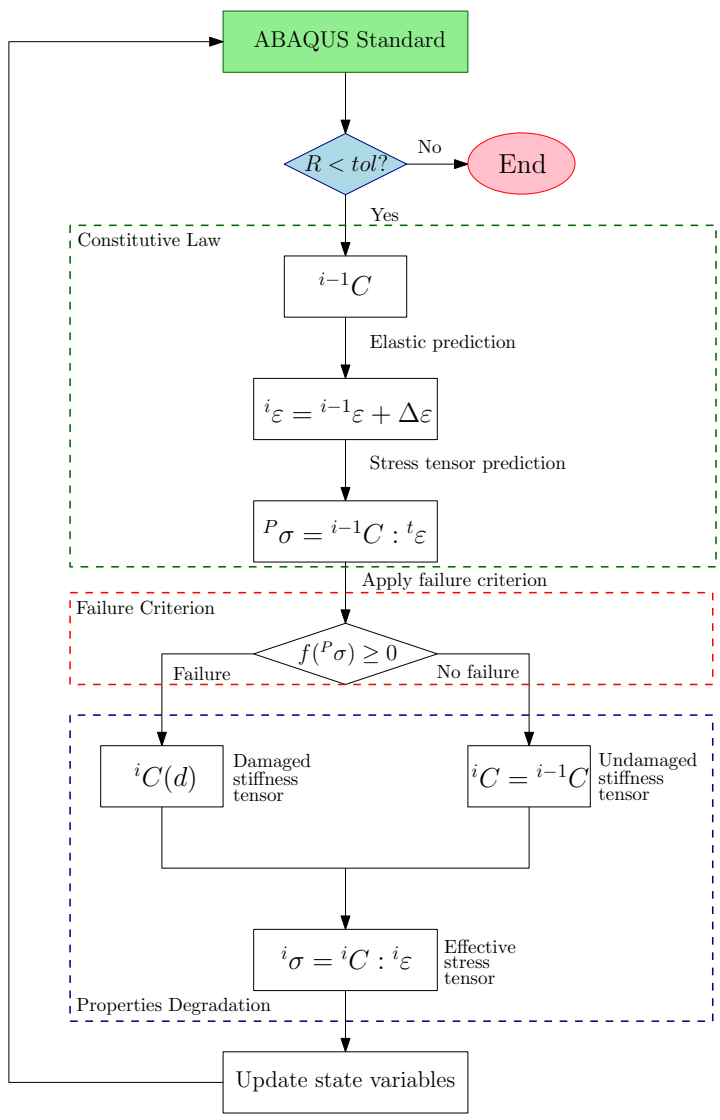

(a)

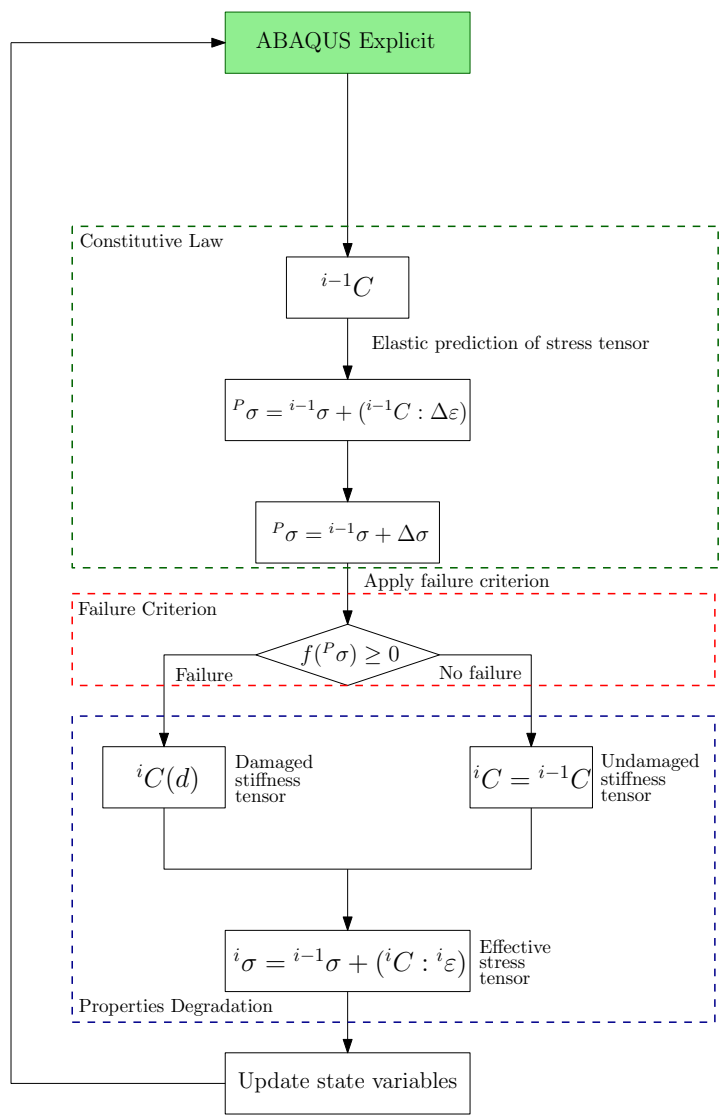

(b)

Source: reconstructed from (FERREIRA, 2014) by the author.

tolerance $($ tol $=0.005)$ and, thus, a new step increment is applied. Otherwise, ABAQUS will finish the simulation. Besides that, it is important to note that in each step increment a reduction of the residue $R$ is expected to characterize convergence; if this does not happen, then divergence occurs and the analysis is terminated by the software.

Regarding to explicit simulations in time, ABAQUS allows VUMAT subroutines implementation. Firstly, the stress tensor ${ }^{P} \sigma$ for a material point is obtained using the previously calculated ${ }^{i-1} \sigma$ stress tensor, its correspondent constitutive matrix ${ }^{i-1} C$ and at the current strain increment step ${ }^{i} \Delta \varepsilon$ provided by ABAQUS. Therefore, that is no need to obtain the Jacobian matrix and the elastic stress tensor prediction is given as ${ }^{i} \sigma={ }^{i-1} \sigma+\left({ }^{i-1} C: \Delta \varepsilon\right)$ in which ${ }^{i-1} C: \Delta \varepsilon=\Delta \sigma$. In this fashion, the constitutive tensor is updated with the values for the state variables previously obtained and, if damage does not occur, these variables are updated with elastic properties values and the elastic constitutive tensor is considered. In the next step, the subroutine verifies the failure criterion condition analogously to the UMAT case. 
It is important to note that in implicit simulations using ABAQUS, the user can simulate virtual monotonic/cyclic quasi-static tests without modelling the mass and/or damping of the structure. Since there are not any parameters of kinetic energy and damping in implicit strategies, when damage occurs and reduction in stiffness is observed, convergence issues can be present as a consequence of discontinuities in the stress-strain relations that occurs due to the sudden reduction on elastic properties, which can lead to a drastic reduction on the time increment or even in analysis interruption. A good strategy to avoid such difficulties is to manually choose a virtual damping parameter. Also, another way is to degrade the properties only at the posterior iteration step aiming analysis continuity. Regarding this last strategy, it is important to choose a sufficiently small iteration step to guarantee good numerical results and is the adopted approach in this work for both implicit and explicit numerical analyses.

For impact analysis, the explicit approach is usually recommended. Moreover, explicit methods are conditionally stable. In this manner, continuity of the numerical solution depends on the adopted time increment, which have to be less than the dilatation wave propagation time in the finite element $(\mathrm{FE})$, and the computational cost is proportional to the size of the FE model (SUN; LEE; LEE, 2000). Thus, it follows that,

$$
\Delta t \leq L^{\mathrm{el}} \sqrt{\frac{\rho}{\lambda+2 \mu}},
$$

has to be satisfied. In Eq. (2.91), $L^{\mathrm{el}}$ is the characteristic length associated to each finite element, $\rho$ is the density, $\lambda$ and $\mu$ are the material Lamé's first and second parameters, respectively. Through this work, since low-energy impacts can be considered as quasi-static indentation tests (TITA, 2003), the implicit strategy using UMAT along with ABAQUS is used.

\subsection{Shear Testing of Composite Materials}

There are various types of distinct standardized test methods to evaluate the behavior of FRP composite materials under in-plane shear state. Each test method has its own specimen geometries and peculiarities with advantages, disadvantages and test devices. The goal of those is of measuring experimentally the shear strength and modulus of the material system even though differences in the overall behavior are observed (LEEUWEN et al., 2008). Therefore, simple test methodologies and specimen manufacture without oversimplifying the problem is required; for example, most methods involves testing the coupon in universal testing machines. Some of the most well known shear test methods are:

- $\left[ \pm 45^{\circ}\right]_{n s}$ tensile (ASTM D3518); 
- Iosipescu (ASTM D5379);

- V-notched rail test (ASTM D7078);

- 2- and 3-rail tests (ASTM D4255).

The first one a shear state is induced in the laminate due to the fibers orientation. The coupon must be symmetric and balanced possessing high aspect ratio. It is one of the most adopted tests since it does not require testing devices, has a high cost-benefit allowing to manufacture many coupons without spending much material and its easy to manufacture (LEE; MUNRO, 1986). One main disadvantage is that as the applied load increases reaching the non-linear region the tendency is that the laminate has its fibers misalignment with the load direction and, thus, some properties such as ultimate shear strength can be not so representative.

The Iosipescu and V-notched rail tests are very similar in procedure and specimen geometry. Both of them require notched laminates to induce failure in this specific region and shear is induced by the load application (compressive) in the test devices as shown in Figure 36 .

Figure 36: Iosipescu and V-notched rail tests.
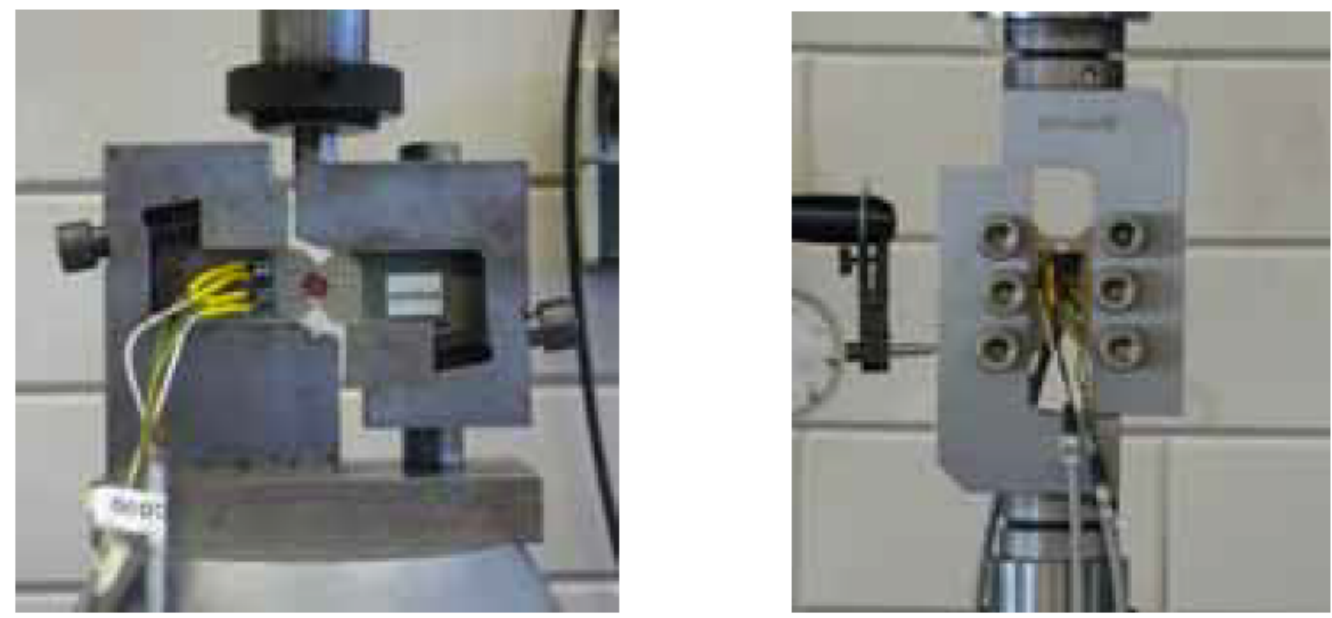

Source: after Leeuwen et al. (LEEUWEN et al., 2008).

Notice that while the Iosipescu test requires a side-loading compression, the v-notched rail requires a face-loading compression. Another difference between both is that for the first one the coupon has a higher aspect ratio with smaller distance between the notches in comparison with the v-notched test coupon. In this sense, one advantage of the last is that it requires less thicker laminates since these, with the specified geometry, are less sensitive to damage that can be introduced by the applied load due to its higher distance between both notches which reduces the influence of the laminate's anisotropy (LEEUWEN et al., 2008). 
Regarding the 2- and 3-rail shear tests, the main difference between both is within the specimen geometry and testing device. Holes are introduced for fixation and loading transference, which generates stress concentrators that can result in premature failure of the laminate affecting mainly the ultimate shear stress value (BAERE; PAEPEGEM; DEGRIECK, 2008). The applied load can be of tension or compression, with the last being the most commonly adopted. Therefore it must be guaranteed that buckling does not occur during testing. Despite these disadvantages, in the case of the 3-rail tests, it is possible to adapt the geometry of the coupon so it can be tested both under rail and drop-weight tests, being so the one chosen in this work for further shear-after-impact analyses. Moreover, the principle of the 3-rail test is depicted in Figure 37 where a pure shear state should be induced in the laminate.

Figure 37: Principle of 3-rail test: setup (a); induced deformation (b).

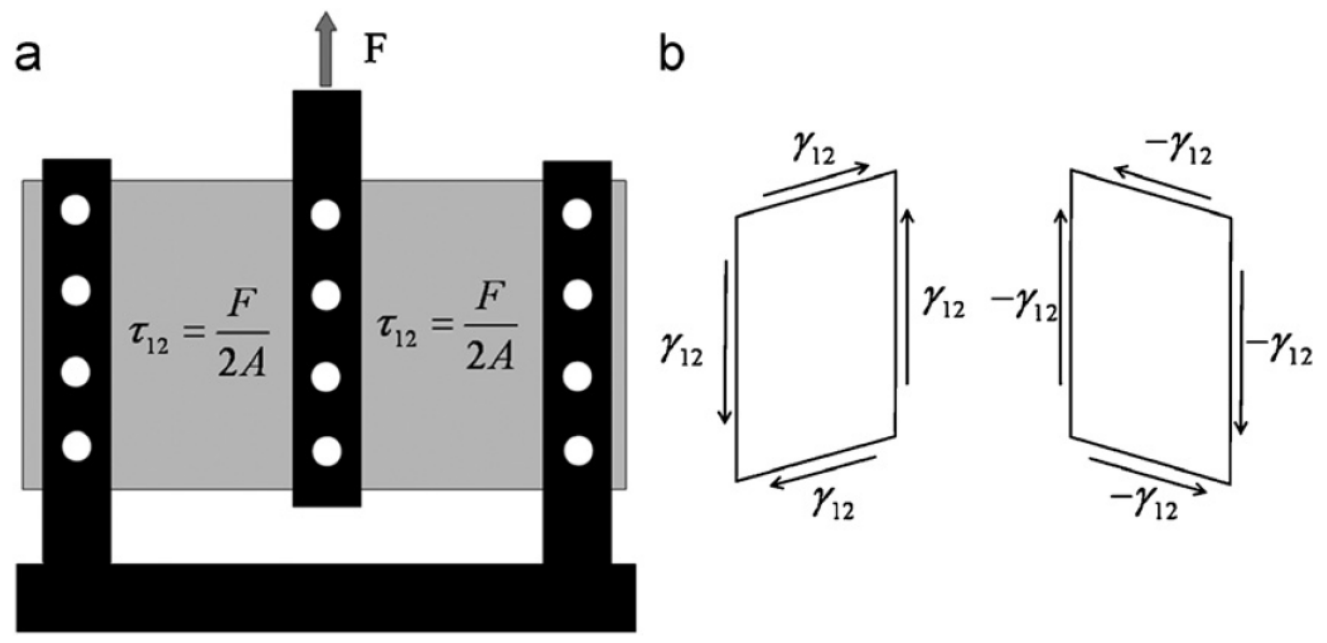

Source: after De Baere, Paepegem and Degrieck (BAERE; PAEPEGEM; DEGRIECK, 2008).

Notice that theoretically the apparatus should induce a pure shear state but what is experimentally observed is that due to the fixation a simple shear state is more in accordance with reality.

It is important to mention that despite the variety of existing test methods there is not a preferable one above all of them. It always depends on the type of analysis that is under study. Also, what is observed between all of them is that the shear modulus slightly varies while the shear ultimate strength presents higher fluctuations. A good example is in the study of Wang, Hao and Ou (WANG; HAO; OU, 2007) in which some laminates shear behaviors are compared under the v-notched rail and $\pm 45^{\circ}$ tensile shear tests as depicted in Figure 38.

In this context, many authors develops their own shear test devices, usually adapted from the standardized ones, for example Adams et al. (ADAMS et al., 2002). Also, attention 
Figure 38: Shear stress-strain curves of L700 laminate.

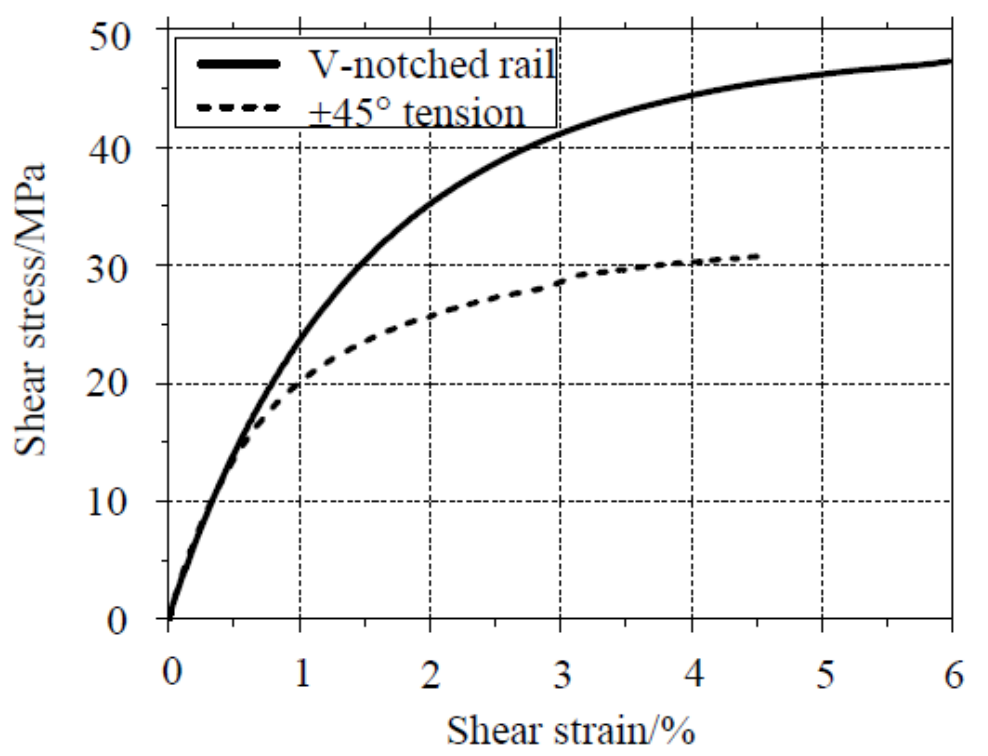

Source: after Wang, Hao and Ou (WANG; HAO; OU, 2007).

must be taken for laminates with high strength since them require very high loads which introduce more damage in the specimens. Furthermore, as aforementioned, there is not one the best shear test method for composites. An interesting benchmark study between various methods is done by Lee and Munro (LEE; MUNRO, 1986) using the decision analysis technique to aid the selection of a test method that better fits one's requirements. In the case of the present work, the 3-rail shear test showed to be the one that have better suitability to the study of the proposed methodology. 



\section{PROPOSED METHODOLOGY}

\subsection{Introduction}

This chapter is dedicated to the study of post-impact (low-energy) methodologies of analysis for composite materials. An overview on the compression-after-impact (CAI) and flexure-after-impact (FAI) is done, where in the last the proposed approach of Medeiros (MEDEIROS, 2016) for FAI is focused. Also, a section dedicated to materials and methods is presented. In it, some experimental results are presented, which are:

- Experimental tests and results for obtaining the fiber and matrix contents of specimens;

- Experimental monotonic quasi-static tests of coupons under tensile and in-plane shear loadings for material characterization;

- Experimental cyclic quasi-static tests of coupons for damage progression evaluation.

Provided with these results and based on the aforementioned post-impact studies, a methodology for the shear after impact analysis is proposed and identification of parameters associated with the material model is performed.

\subsection{Post-Impact Methodologies for Composite Materials}

To overcome over-dimensioning practices due to impact sensitivity of composite materials, there is a high demand in high-performance industry (mainly aeronautical and aerospace) for the assessment of the post-impact behavior and residual properties of structures composed by these materials. In this sense, it is of unique importance to demonstrate that these structures are still able to sustain loads after an impact event, regardless of its cause (BOUVET; RIVALLANT, 2016). Additionally, prediction of damage extension, severity and evolution is also extremely important. The understanding of these concepts leads to less-conservative design philosophies such as damage tolerance. Nowadays, there are still several drawbacks that delays its appliance in industry and overcoming these represents great advance in the context of sustainable development.

\subsubsection{Compression-After-Impact}

The most affected characteristic of composite laminates subjected to impact is the compressive strength mainly due to buckling (ABRATE, 1998; THORSSON; WAAS; RASSAIAN, 2018a; BOUVET; RIVALLANT, 2016) leading up to $75 \%$ of strength loss (BOUVET; RIVALLANT, 2016) in some cases. Commonly, compression-after-impact 
testing is carried out in laminated composite plates subjected to low-energy impact that induces delamination. Thus, its application usually lies on impacted cross-ply laminates. Once under compression, the impacted laminate can experience local, global or mixed buckling modes over the delaminated region. Thus, the buckling of sub-laminates delaminated during impact can occur inducing growth of impact induced delaminations with load application which leads to increase of buckling even more throughout the laminate. Usually, this phenomenon is catastrophic culminating in total failure of the laminate with delamination growth oriented perpendicularly with the loading direction. Meanwhile, compressive fiber failure growth of plies oriented with the load direction is also observed (BOUVET; RIVALLANT, 2016). Figure 39 shows a typical scenario of damage and failure under CAI.

Figure 39: Typical scenario of damage and failure under CAI.

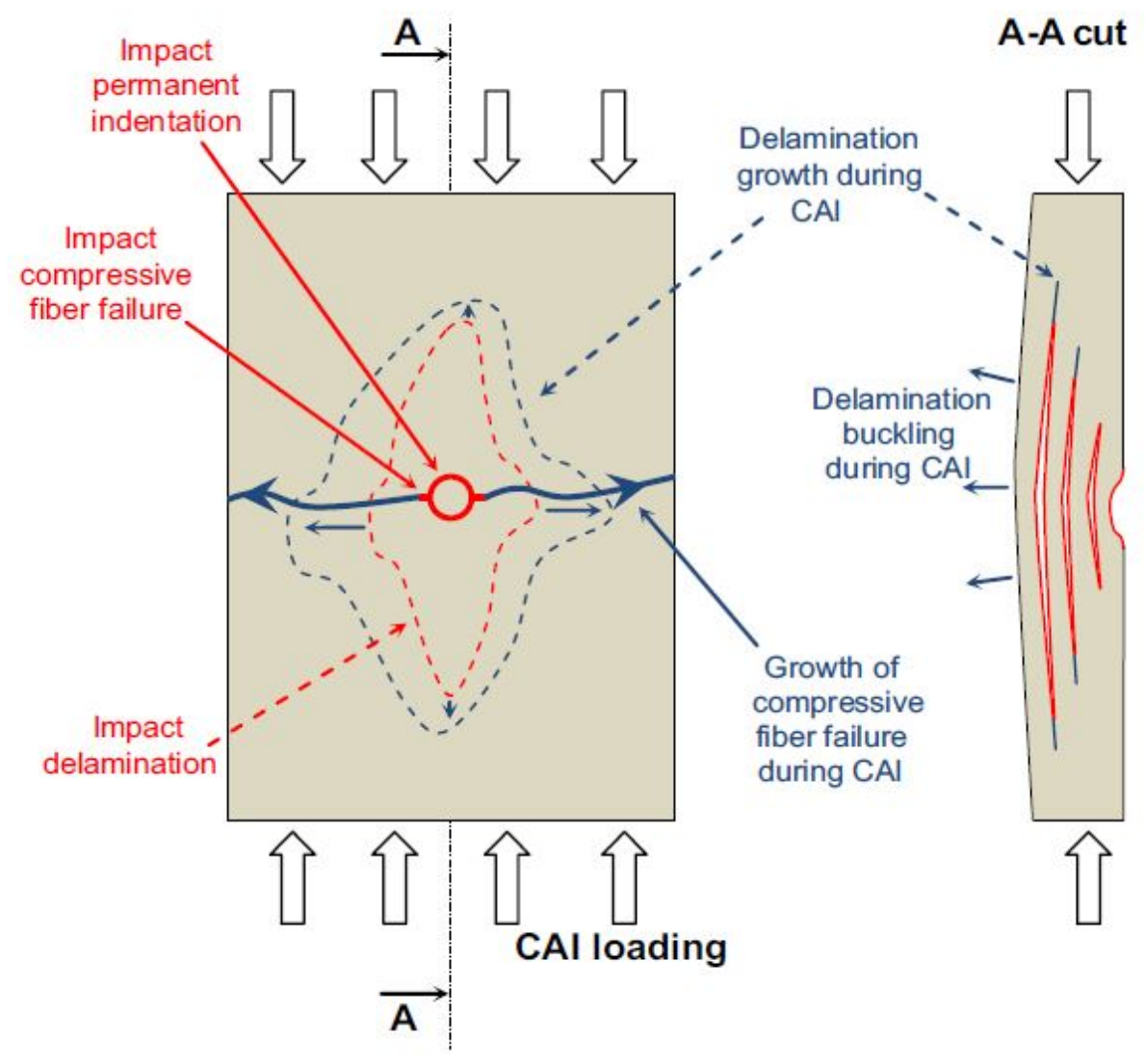

Source: after Bouvet and Rivallant (BOUVET; RIVALLANT, 2016).

It is important to highlight that the CAI experiment is a component test and it is not recommended to be done. This means that its objective is not to measure any material property but to collect data to be used as a sound basis for comparison with consistent specimen dimensions and test conditions (HODGKINSON, 2000). Regarding to the experimental apparatus, CAI tests can be performed in universal testing machines 
such as the ones by MTS or INSTRON, with the specimen fixture represented in Figure 40 as specified by the ASTM D7137 standard.

Figure 40: Compression-after-impact support fixture.

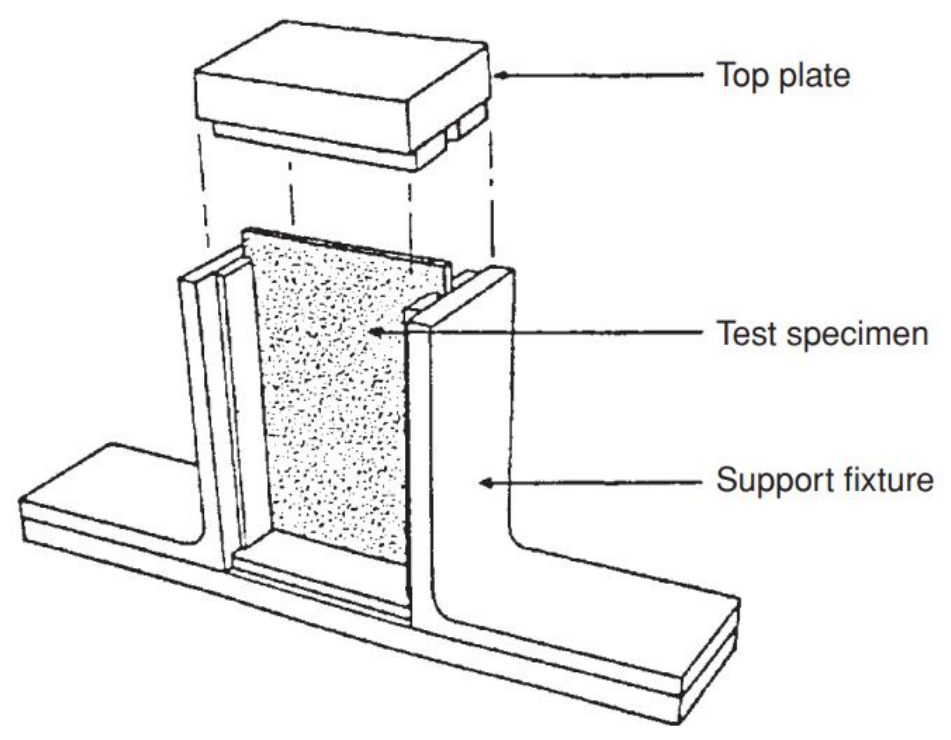

Source: American Society for Testing and Materials.

As can be noticed, the support restrains the vertical sides of the sample with anti-buckling rails equipped by knife edge supports. Also, there is a small portion of the specimen that is unsupported by the side rails of the fixture enabling a maximum compressive strain of approximately 3.3\% before excessive deflections occurrence. In time, it is important to mention that residual strength decreases with increasing impact energy and, consequently, with growing damage extension (HODGKINSON, 2000).

\subsubsection{Flexure-After-Impact}

Flexure-after-impact analysis is usually based on the evaluation of strength limit and stiffness loss experimentally assessed by 3- or 4-point bending tests. In 3-point bending, the maximum longitudinal fiber stress is located bellow the loading nose which produces stress concentration and develops undesirable cracks in the specimen. On the other hand, in 4-point bending constant load and uniformity of the stress distribution through specimen's span is observed. Thus, the last approach is more appropriate to coupons that experiences great displacements. Moreover, it is worth to mention that FAI literature is, in some sense, limited in comparison with the CAI one since there are more studies dedicated to CAI than FAI.

Among the existent FAI works, the one by Medeiros (MEDEIROS, 2016) is of particular importance. Based on the above-mentioned reasons, an investigation of post-impact strength of CFRP is experimentally performed by means of 4-point bending 
tests. During these, compression loads are strategically induced in the impacted side of the specimen since the objective of the study was to propose a new methodology to serve as an alternative to CAI approach. Also, the identification and localization of impact damage are done by non-destructive methods, namely: vibration based method (VBM) and shearography speckle (SS), respectively. The residual strength is assessed by means of a damage index (DI) which is based in FAI results. This damage index is Classical Laminate Theory (CLT) based, thus, it follows Kirchhoff's kinematics hypotheses and the laminate is considered to experience only plane stress states. Also, the main goal of the damage index is to answer the following questions:

- The structure still possess strength to support post-impact loads?

- It is necessary to repair the structure?

Considering the constitutive equation of multi-directional laminates (repeated here) given as,

$$
\left\{\begin{array}{l}
\underline{\mathrm{N}} \\
\underline{\mathrm{M}}
\end{array}\right\}=\left[\begin{array}{ll}
\mathbf{A} & \mathbf{B} \\
\mathbf{B} & \mathbf{D}
\end{array}\right]\left\{\begin{array}{c}
\underline{\varepsilon}^{0} \\
\underline{\kappa}
\end{array}\right\},
$$

under 4-point bending, the only non-zero variables in Eq. (3.1) are $M_{x}$ and $\kappa_{x}$, so it reduces to

$$
M_{x}=D_{11} \kappa_{x}
$$

Now, following Medeiros (MEDEIROS, 2016) approach, considering the maximum bending moment that the pristine structure can hold $\left(M_{\max }^{P}\right)$ and the maximum bending moment that the damaged structure holds $\left(M_{\max }^{D}\right)$, the damage index is introduced as,

$$
D I_{\mathrm{FAI}}:=1-\frac{M_{\max }^{D}}{M_{\max }^{P}} .
$$

Thus, when $D I=1$ the structure is fully damaged, $D I=0$ holds for no noticeable damage and when $0<D I<1$ it means that the structure is partially damaged.

\subsection{Materials and Methods}

The laminated composite plates used in this work for material characterization, impact and post-impact tests were all manufactured from a pre-impregnated (with epoxy resin system) unidirectional carbon fiber fabric from Texiglass Industry and Textile Commerce with nominal areal weight of $208 \mathrm{~g} / \mathrm{m}^{2}$ and $0.29 \mathrm{~mm}$ of nominal thickness. Also, the series of plates are manufactured using hand-layup technique inside a vacuum bag following a sequence of systematic proceedings to guarantee reliability and reproducibility of the laminates and results. As lamination sequence it is adopted: 
1. Thick glass plate used as mold for the laminates preventing warping of them during the cure cycle;

2. Teflon film (A) to make the cured laminate withdraw easier;

3. Pre-impregnated carbon plies each of which with its own orientation angle depending of the final purpose of the laminate;

4. Perforated film (B) to control the precipitation flux of resin during the cure cycle;

5. Peel-ply film (C) for resin excess absorption;

6. Pressure redistribution mesh (F) for homogenization of vacuum inside the vacuum bag;

7. Breather cloth layer (E) to allow conducting volatile components and trapped air inside the vacuum bag to the vacuum pump connector;

8. Metallic plate to serve as aid for laminate molding;

9. Another breather cloth layer;

10. Vacuum bagging film sealed with appropriate sealant tape;

11. Vacuum connector (F).

Regarding to the vacuum bag, it creates a difference of pressure between the inside and outside environments of the bag, enforcing compaction of it. This compaction pressure gives and maintain the mold form to the laminate and also induces excess of gases and volatile components elimination during cure. It helps to distribute in homogeneity the resin in the reinforcement and to bleed it excess to improve the fiber, matrix and void fraction ratios. Due to Torricelli's Principle, the maximum possible achievable pressure is equal to 1 atmosphere if no air insertion exists in the vacuum bag (i.e. perfect sealing). Thus, some manufacturers likes to use autoclaves to improve this maximum vacuum level. In this work, the process occurs without it.

The choice for vacuum bagging proceeding is done because is one of the most cost benefit approaches and is largely used in industry being considered a relatively reliable process since it depends on the technician's ability and experience. This highlights even more the importance of following the exposed lamination sequence. Figure 41a presents the micro-controlled oven while Figures $41 \mathrm{~b}$ and 41c presents each of the lamination components and their assembly.

The aforementioned cure is the process in which modification of the physical properties of the polymer matrix (or simply, resin) occur making it evolve to the solid state. When this state is reached, cross-linking of the reticulated molecular structure is 
Figure 41: Oven for curing (a), adopted lamination sequence with required components (b) and vacuum bag scheme (c).

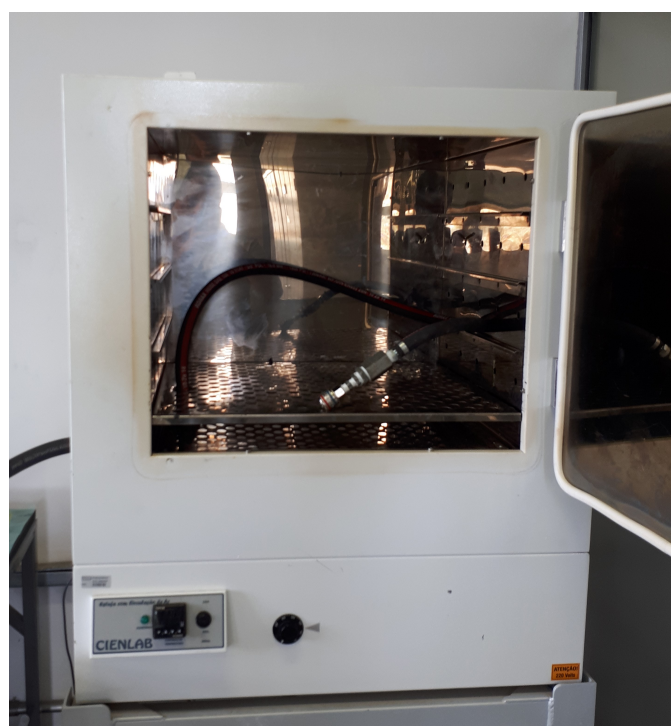

(a)

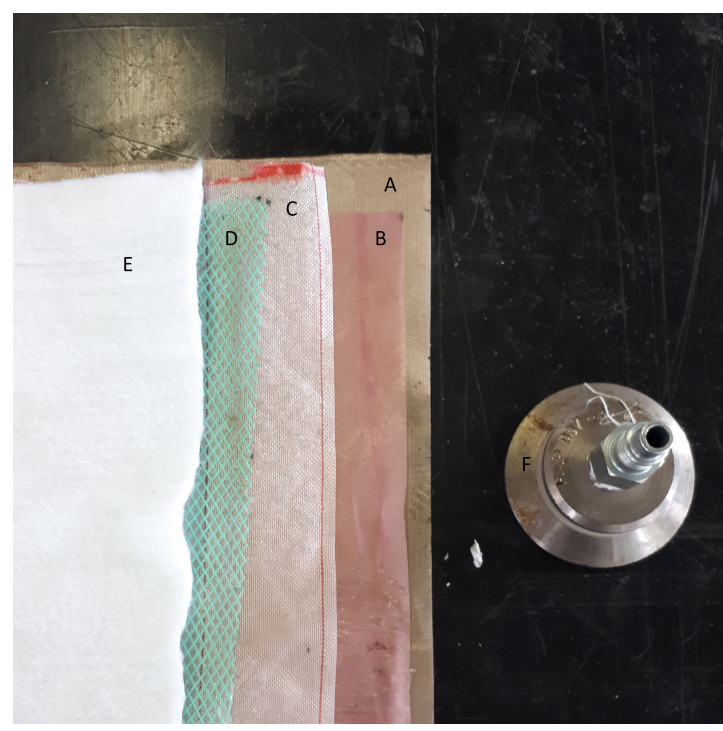

(b)

To vacuum source (air and volatiles)

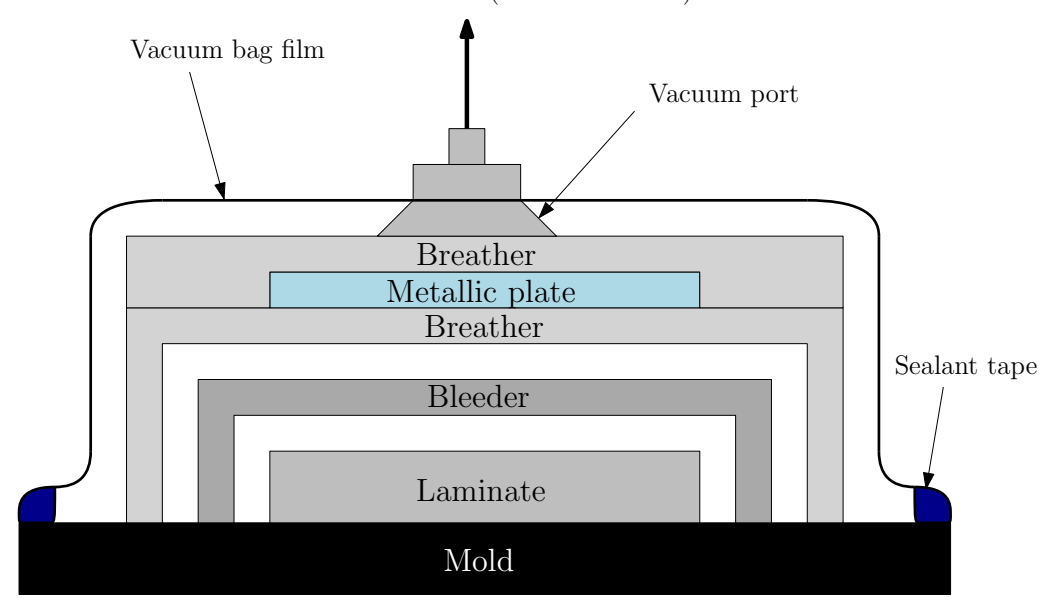

(c)

Source: by the author.

observed. Thus, in this work, the thermofix type of polymer matrix is used and, therefore, it cannot be reworked after cure. The cure cycle is the procedure in which the matrix has to go through to cure. In it, it is defined how many time and at which temperature the resin must stay to reach cure. Usually, resins tends to cure faster when submitted to high temperature levels for some time period. This procedure accelerate the process but also tends to input some residual stresses in the laminate due to the temperature gradient and higher change in volume of it during the process. Thus, it is important to program the cure cycle with smooth temperature ramps, to mitigate these effects. In this work, the manufacturer recommended cure cycle is adapted to account these drawbacks since all 
laminates are submitted to cure with heat inside an oven. Figure 42 presents the cure cycles of the manufactured composite laminates.

Figure 42: Expected and experimental cure cycles of the composite laminated plates.

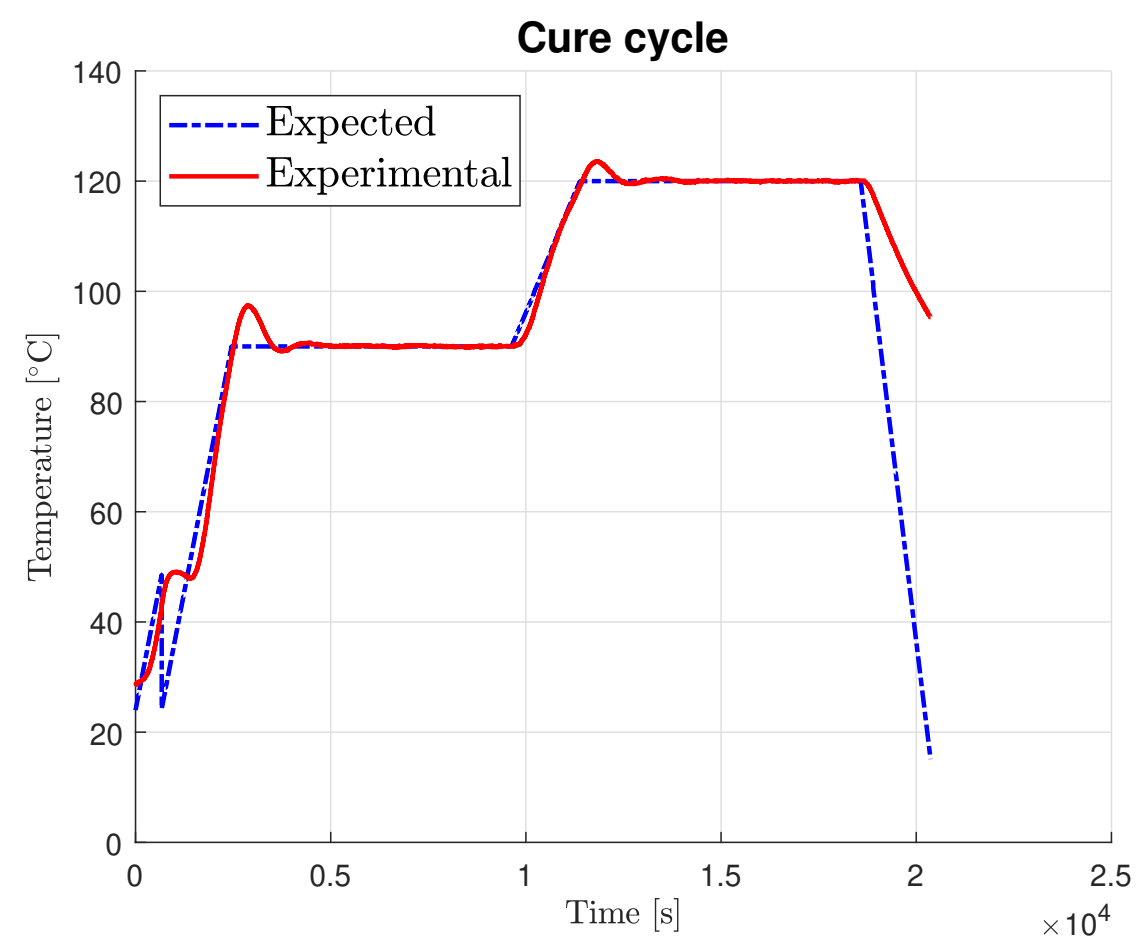

Source: by the author.

As can be noticed, good agreement between the theoretical and experimental curves is achieved producing well-cured laminates as expected with the pressure during the cure cycles remained almost constant around 23.0 in $/ \mathrm{Hg}$. Each plateau temperature was maintained for a period of 2 hours and each temperature ramp was maintained for a period of 30 minutes summing up 5 hours and 30 minutes of curing time. Moreover, as can be noticed, there is no good agreement between the expected and theoretical curves in the last temperature ramp. This is due to the non-controlled cooling adopted strategy; the cool-down was performed by leaving the specimen reach room temperature by itself. Also, as aforementioned, high temperature gradients can be harmful during the cure process. Thus, a first plateau temperature is maintained with the objective of mitigate this effect. Lastly, some manufacturing guidelines for good-practice are proposed as follows:

- Material availability checking;

- Vacuum pump and oven testing;

- Mold cleaning with isopropyl alcohol;

- Bleed, breather and vacuum bag material cutting; 
- Removal of the pre-impregnate from the freezer (to let it reach room temperature) and cutting in appropriate dimensions;

- Weighting with precision balance and measurement of ply thickness;

- Documentation;

- Vacuum bag assembly;

- Vacuum leakage and quality checking;

- Execution of the cure cycle at the oven;

- Organization of the work place and utilized material cleaning;

- Laminated plate withdraw, weighting and finishing (corners cutting and sanding);

- Specimen final thickness measurement;

- Coupons cutting, abnormalities documentation and cataloging.

By following these guidelines, work conditions should be favorable to manufacture adequate coupons for experimental testing.

\subsubsection{Fiber and Matrix Contents}

Measurement of fiber $V_{f}$ and matrix $V_{m}$ volume fractions are important for assessing the quality of the adopted manufacturing process. Therefore, tests are performed in accordance with the ASTM D3529 standard (Procedure B) for obtaining these properties. In it, the composite prepreg is exposed to a high temperature value in an air environment for a long time period. Table 4 shows the obtained results for each tested coupon.

Table 4: Obtained results for fiber/matrix volume fractions of each coupon

\begin{tabular}{lrrrrr}
\hline Coupon & Initial mass $[\mathrm{g}]$ & Final mass $[\mathrm{g}]$ & Mass \% of matrix & Matrix volume fraction & Fiber volume fraction \\
\hline $\mathrm{C} \# 1$ & 2.180 & 1.524 & 0.3009 & 0.386 & 0.614 \\
$\mathrm{C} \# 2$ & 1.830 & 1.365 & 0.2541 & 0.332 & 0.667 \\
$\mathrm{C} \# 3$ & 1.740 & $\mathrm{n} / \mathrm{a}$ & $\mathrm{n} / \mathrm{a}$ & $\mathrm{n} / \mathrm{a}$ & $\mathrm{n} / \mathrm{a}$ \\
\hline
\end{tabular}

For these tests, the coupons were exposed to $500^{\circ} \mathrm{C}$ air temperature during three hours inside an oven with $5^{\circ} \mathrm{C} / \mathrm{min}$ of heating rate. As can be noticed, coupon $\# 3$ has no documented results since it was verified reinforcement loss during the process. Table 5 shows some properties used for the calculations and average values for the volume ratios.

As can be noted, the differences $(\Delta V)$ between the obtained results for the tested coupons are of $5.4 \%$ and $5.3 \%$ for matrix and fiber volume fractions, respectively.

$\overline{2}$ The unit $\mathrm{g} / \mathrm{cc}$ stands for grams per cubic centimeter. 
Table 5: Fiber and matrix volume fractions final results

\begin{tabular}{lrrr}
\hline Property & Unit & Value & Procedure \\
\hline Mean matrix volume fraction & $\mathrm{n} / \mathrm{a}$ & 0.359 & ASTM D3529 \\
Mean fiber volume fraction & $-"-$ & 0.641 & $-"-$ \\
Reinforcement density & $\mathrm{g} / \mathrm{cc}^{2}$ & 1.9 & Manufacturer data \\
Matrix density & $-"-$ & 1.3 & $-"-$ \\
\hline
\end{tabular}

Considering the manufacturing process, this reveals that the hand-layup adopted procedures yields relatively good specimens for further testing.

\subsubsection{Monotonic Quasi-Static Material Characterization Tests}

A test campaign for material characterization following the adequate standards was performed. According to Ferreira (FERREIRA, 2014), the Digital Image Correlation (DIC) technique can substitute other displacement data acquiring methods to measure the strain state in the specimens during the tests. In his work, a comparison with DIC and strain gages data is performed and little deviation is observed between them. Since this approach is more cost benefit, in this work the DIC technique is also adopted. Therefore, specimens are sprayed with black paint in a white background creating a random pattern of dots as depicted in Figure 43a.

Figure 43: Example of a coupon after spraying (a) and universal testing machine (b).

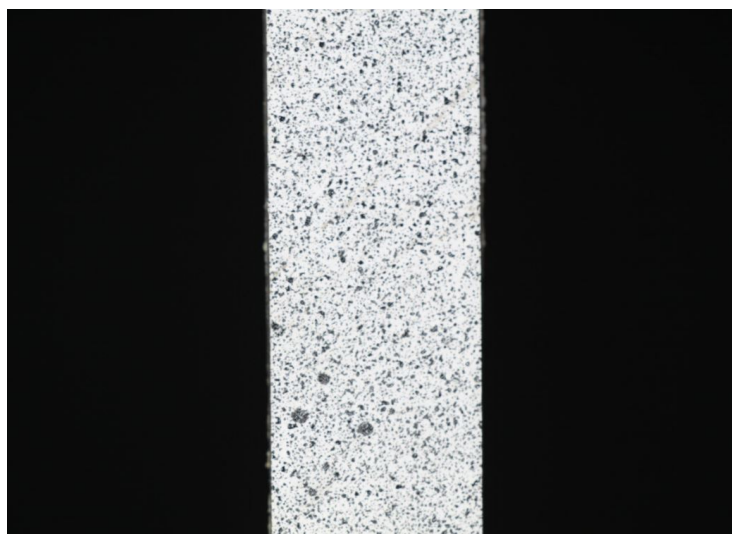

(a)

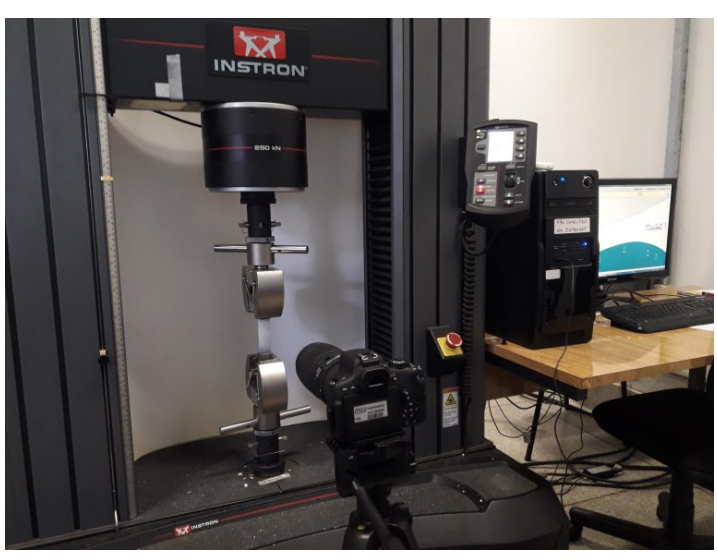

(b)

Source: by the author.

While taking pictures of these points during the tests, the GOM Correlate software process the images obtaining the displacement field and its gradient, making possible to obtain the strain field in a representative area of the coupon. The adopted picture acquirement system consists of a digital camera (CANON EOS 350) connected to a 
computer. All tests are performed in a INSTRON 5985 (Figure 43b) universal testing machine system with $250 \mathrm{kN}$ loading capacity.

Tensile tests were performed following ASTM D3039 standard procedures. The type of control used for the machine cross-head was displacement based with a testing speed of $2 \mathrm{~mm} / \mathrm{min}$. Besides it, for every characterization three specimens are tested, accordingly to the minimum representative quantity of tests imposed by the standard. The longitudinal tensile test measures the following properties:

- Longitudinal elasticity modulus $E_{11}$;

- Longitudinal tensile strength $X_{T}$;

- Major Poisson ratio $\nu_{12}$;

- Longitudinal tensile strain limit $\varepsilon_{1 T}$.

The first, is given as,

$$
E_{11}=\frac{\Delta \sigma}{\Delta \varepsilon}
$$

where $\Delta \sigma$ is the stress difference measured at 1000 and $3000 \mu \varepsilon$ strain and $\Delta \varepsilon$ is the strain difference that assumes a nominal value of $2000 \mu \varepsilon$. Following, the longitudinal tensile strength is,

$$
X_{T}=\frac{P_{\max }}{A}
$$

with $P_{\max }$ the maximum load before failure and $A$ the cross-section average value of the specimen (width and thickness are measured more than on time). The major Poisson ratio is calculated by,

$$
\nu_{12}=-\frac{\Delta \varepsilon_{T}}{\Delta \varepsilon_{L}}
$$

in which $\Delta \varepsilon_{T, L}$ are the strain differences in the transverse $(\mathrm{T})$ and longitudinal $(\mathrm{L})$ directions, respectively. The last property is taken simply as being the corresponding strain at fracture stress values. Figure 44 shows the obtained stress-strain curves for each $\left[0^{\circ}\right]_{8 T}$ coupon.

As can be readily observed, the three specimens presented similar trending curves and results. To evaluate these systematically, statistical treatment to data is performed. Firstly, the average value $\bar{x}$ is taken by,

$$
\bar{x}=\frac{1}{n} \sum_{i=1}^{n} x_{i}
$$


Figure 44: Stress-strain curves for $\left[0^{\circ}\right]_{8 T}$ coupons under tension.

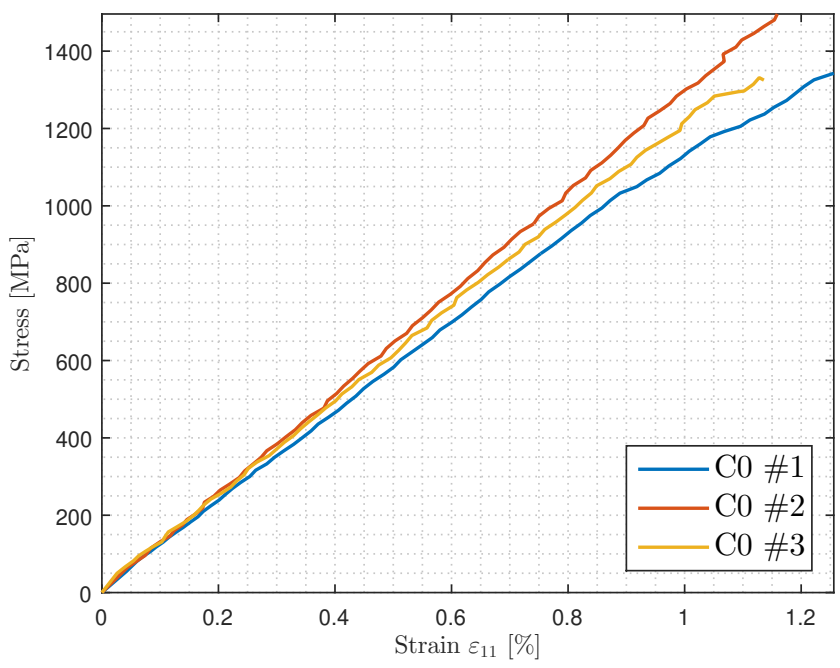

Source: by the author.

with $n$ and $x_{i}$ the number of specimens and measured values during tests, respectively. Next, the standard deviation $S_{n-1}$ and coefficient of variation $\mathbf{C V}$ are calculated as,

$$
\begin{aligned}
& S_{n-1}=\sqrt{\frac{1}{n-1} \sum_{i=1}^{n}\left(x_{i}^{2}-n \bar{x}^{2}\right)}, \\
& \mathbf{C V}=\frac{S_{n-1}}{\bar{x}} \times 100 .
\end{aligned}
$$

Finally, the confidence interval IC is calculated. Considering a symmetrical IC of $95 \%$ based in a $t$-distribution value, it follows,

$$
\mathbf{I C}=\bar{x} \pm t_{n-1(2.5 \%)} \frac{S_{n-1}}{\sqrt{n}} .
$$

The obtained results for the longitudinal tensile tests are shown in Table 6 .

Table 6: Results for the longitudinal tensile tests of $\left[0^{\circ}\right]_{8 T}$ specimens

\begin{tabular}{lrrrr}
\hline Coupon & $E_{11}[\mathrm{GPa}]$ & $\nu_{12}$ & $X_{T}[\mathrm{MPa}]$ & $\varepsilon_{1 T}$ \\
\hline C0 \#1 & 115.10 & 0.255 & 1343.71 & 0.01256 \\
C0 \#2 & 130.24 & 0.307 & 1502.23 & 0.01159 \\
C0 \#3 & 121.65 & 0.298 & 1366.31 & 0.01136 \\
\hline Average & 122.33 & 0.287 & 1404.08 & 0.01184 \\
$S_{n-1}$ & 7.59 & 0.046 & 85.74 & 0.00156 \\
CV $[\%]$ & 6.21 & 16.21 & 6.11 & 5.38 \\
IC & \pm 18.86 & \pm 0.116 & \pm 213.0 & \pm 0.00156 \\
\hline
\end{tabular}

Notice that sample $\mathrm{C} 0 \# 2$ reached higher properties than the others. The reason for this is probably due to the manufacture process that is high dependent on the experience 
of the technician and handling and must be done carefully to avoid results fluctuations. Regarding to transverse tensile tests, these were performed in $\left[90^{\circ}\right]_{8 T}$ coupons in similar conditions of the longitudinal tests. The objective is to obtain the following properties:

- Transverse elasticity modulus $E_{22}$;

- Transverse tensile strength $Y_{T}$;

- Transverse tensile strain limit $\varepsilon_{2 T}$.

Figure 45 shows the obtained stress-strain curves.

Figure 45: Stress-strain curves for $\left[90^{\circ}\right]_{8 T}$ coupons under tension.

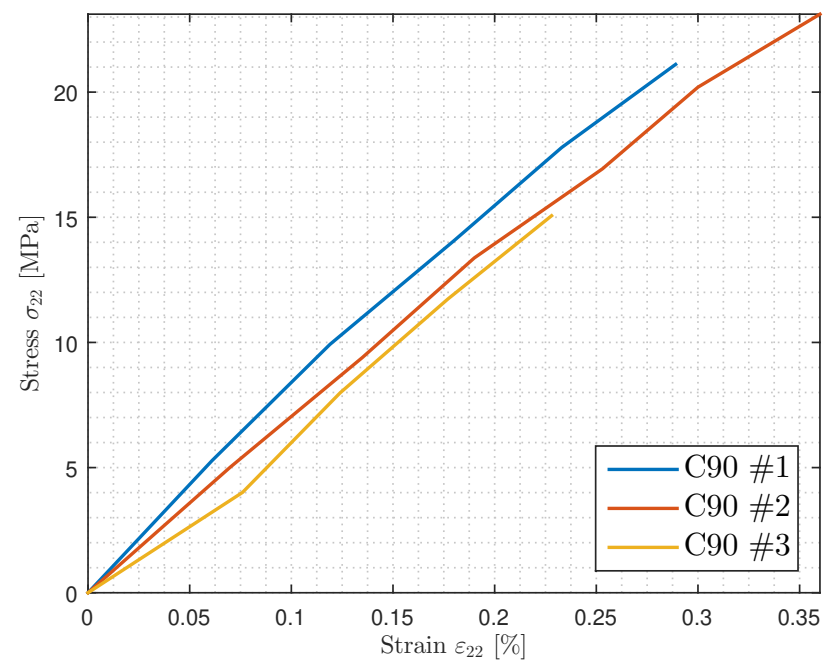

Source: by the author.

Analogously, Table 7 shows the obtained results with statistical treatment.

Table 7: Results for the transverse tensile tests of $\left[90^{\circ}\right]_{8 T}$ specimens

\begin{tabular}{lrrr}
\hline Coupon & $E_{22}[\mathrm{GPa}]$ & $Y_{T}[\mathrm{MPa}]$ & $\varepsilon_{2 T}$ \\
\hline C90 \#1 & 7.30 & 21.82 & 0.00289 \\
C90 \#2 & 6.42 & 25.78 & 0.00360 \\
C90 \#3 & 6.61 & 17.03 & 0.00228 \\
\hline Average & 6.78 & 21.55 & 0.00292 \\
$S_{n-1}$ & 0.47 & 4.38 & 0.00066 \\
CV [\%] & 6.87 & 20.32 & 22.59 \\
IC & \pm 1.16 & \pm 10.88 & \pm 0.00164 \\
\hline
\end{tabular}


With the obtained results, the minor Poisson ratio $\nu_{21}$ can be obtained by (JONES, 1999),

$$
\nu_{21}=\frac{E_{22}}{E_{11}} \nu_{12}
$$

due to the symmetry of the stiffness matrix. Thus, considering the collected mean values, it follows that $\nu_{21}=0.0159 \pm 0.0071$. For shear properties, the ASTM D3518 standard was used. According to it, the following can be obtained by performing tensile tests in $\left[ \pm 45^{\circ}\right]_{4 s}$ coupons.

- In-plane shear modulus $G_{12}$;

- In-plane shear strength $S_{12}$;

- Ultimate in-plane shear strain $\bar{\gamma}_{12}$.

The first is obtained by,

$$
G_{12}=\frac{\Delta \sigma_{12}}{\Delta \gamma_{12}}
$$

where $\Delta \sigma_{12}$ corresponds to the difference in measured applied shear stress between the two shear strain points $\Delta \gamma_{12}$ assuming a nominal value of 0.004 . The next, corresponds to

$$
\sigma_{12}=\frac{P_{i}}{2 A}
$$

that for the strength one needs to pick the maximum stress value obtained at the i-th data point. Lastly, the strain limit is obtained by,

$$
\bar{\gamma}_{12}=\varepsilon_{x x}-\varepsilon_{y y}
$$

in which $\varepsilon_{x x}$ and $\varepsilon_{y y}$ corresponds to the longitudinal and transverse normal strains at failure, respectively. The stress-strain obtained curves are shown in Figure 46.

Similarly, the adequate statistical treatment is done with the obtained results and are shown in Table 8.

Table 8: Results for the shear tests of $\left[ \pm 45^{\circ}\right]_{4 s}$ specimens

\begin{tabular}{lrrr}
\hline Coupon & $G_{12}[\mathrm{GPa}]$ & $S_{12}[\mathrm{MPa}]$ & $\bar{\gamma}_{12}$ \\
\hline C45 \#1 & 3.52 & 34.91 & 0.0149 \\
C45 \#2 & 3.35 & 38.79 & 0.0246 \\
C45 \#3 & 3.55 & 39.44 & 0.0165 \\
\hline Average & 3.47 & 37.71 & 0.0186 \\
$S_{n-1}$ & 0.11 & 2.45 & 0.0052 \\
CV [\%] & 3.20 & 6.49 & 27.75 \\
IC & \pm 0.28 & \pm 6.09 & \pm 0.0129 \\
\hline
\end{tabular}


Figure 46: Stress-strain curves for $\left[ \pm 45^{\circ}\right]_{4 s}$ coupons under tension.

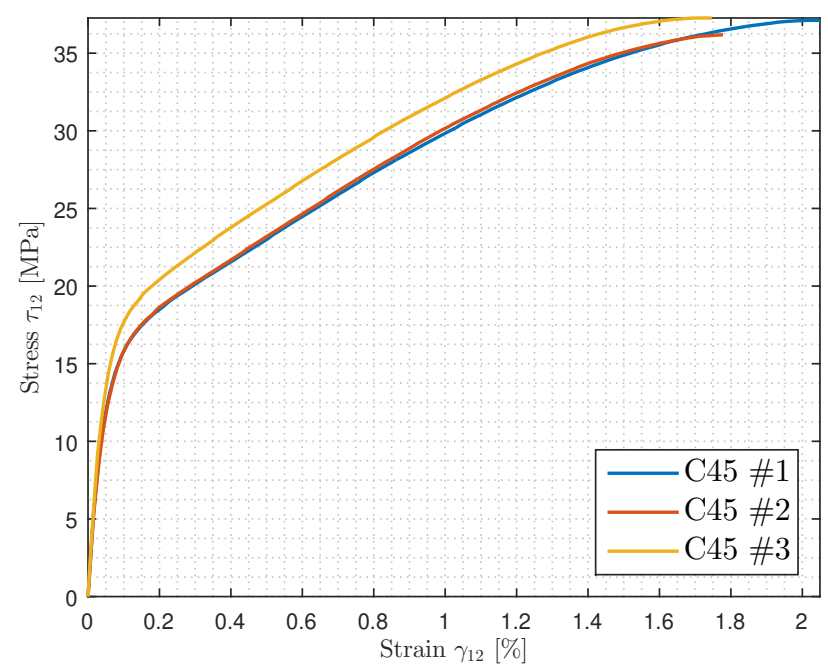

Source: by the author.

\subsubsection{Summary of Monotonic Quasi-Static Tests Results}

To simplify visualization and further consultation, Table 9 provides a summary of the monotonic quasi-static tests obtained results. Notice that since the material model has a bi-dimensional nature, there are no results for out of plane elastic and strength values. Nevertheless, some of these properties can also be used if needed, since they come from material symmetry. Also, the composite density $\rho_{c}$ is obtained applying the rule of mixtures.

Table 9: Carbon fiber composite properties summary

\begin{tabular}{cccccc}
\hline \multicolumn{3}{c}{ Elastic properties } & \multicolumn{3}{c}{ Strength values } \\
\hline$E_{11}[\mathrm{GPa}]$ & 122.33 & $127^{(i i)}$ & $X_{T}[\mathrm{MPa}]$ & 1404.08 & $1400^{(i i)}$ \\
$E_{22}[\mathrm{GPa}]$ & 6.78 & $10^{(i i)}$ & $Y_{T}[\mathrm{MPa}]$ & 21.55 & $47^{(i i)}$ \\
$G_{12}[\mathrm{GPa}]$ & 3.47 & $5.4^{(i i)}$ & $S_{12}[\mathrm{MPa}]$ & 37.71 & $53^{(i i)}$ \\
$\nu_{12}$ & 0.287 & $0.34^{(i i)}$ & $S_{13}[\mathrm{MPa}]$ & $-"-$ & $53^{(i i)}$ \\
\hline \multicolumn{3}{c}{ Physical properties } & $\rho_{c}[\mathrm{~g} / \mathrm{cc}]$ & 1.685 & $1.58^{(i i)}$ \\
& & $V_{f}[\%]$ & 64.1 & $63^{(i i)}$ \\
\hline
\end{tabular}

(ii): Hexcel M40J prepreg (TITA, 2003)

Comparing the obtained results it is noticeable that the matrix dependent properties from the Texiglass prepreg are much weaker than those from Hexcel. By the other hand, physical and fiber dependent properties are in good agreement. This leads us to believe that the pre-impregnated epoxy resin system is of lower quality in comparison with the one used by Tita and/or its storage was not properly carried out. 


\subsubsection{Cyclic Quasi-Static Tests and Material Model Parameters Identification}

If monotonic tests provides information about damage onset, then cyclic tests raise data about damage evolution. Table 10 summarize all the parameters that needs identification and corresponding standards used for doing it for both test types.

Table 10: Tests and standards for material parameters identification

\begin{tabular}{lllr}
\hline Testing coupon & Resulting Parameters & Test type & Standard \\
\hline Tensile $0^{\circ}$ & $E_{11}, \nu_{12}, X_{T}$ & Monotonic & ASTM D3039 \\
Tensile $90^{\circ}$ & $E_{22}, Y_{T}$ & Monotonic & $\mathrm{n} / \mathrm{a}$ \\
\hline & $d_{2}, \sqrt{Y_{0}^{\prime}}, \sqrt{Y_{C}^{\prime}}$ & Cyclic & ASTM3518 \\
\hline In-plane shear & $G_{12}, S_{12}, S_{12_{y}}$ & Monotonic & $\mathrm{n} / \mathrm{a}$ \\
(tensile $\left.\pm 45^{\circ}\right)$ & $d_{6}, \sqrt{Y_{0}}, \sqrt{Y_{C}}$ & Cyclic & ASTM D3410 \\
\hline Compressive $0^{\circ}$ & $X_{C_{0}}, X_{C}, f\left(\varepsilon_{11}\right)$ & Monotonic & AS \\
Compressive $90^{\circ}$ & $Y_{C_{0}}, Y_{C}, g\left(\varepsilon_{22}\right)$ & & $\mathrm{n} / \mathrm{a}$ \\
\hline Tensile $45^{\circ}$ & $d_{2}, d_{6}, b$ & Cyclic & \\
Tensile $70^{\circ}$ & $d_{2}, d_{6}, b$ & & \\
\hline
\end{tabular}

Firstly, since there is any compression test apparatus at the GEA facilities the related parameters obtained via these tests used in this work for the material model computational application are the same as those obtained by Ferreira (FERREIRA, 2014), since it was studied a very similar material in comparison with the one investigated through the present text. Thus, using the procedure described in Figure 23, the obtained parameters are listed in Table 11.

Table 11: Compression damage modes parameters

\begin{tabular}{llr}
\hline Ply damage mode & Linear-elastic threshold stress & Strain function \\
\hline Compression in direction 1 & $X_{C 0}=253 \mathrm{MPa}$ & $f\left(\varepsilon_{11}\right)=78.873 \varepsilon_{11}+1.087$ \\
Compression in direction 2 & $Y_{C 0}=60.3 \mathrm{MPa}$ & $g\left(\varepsilon_{22}\right)=4.1763 \varepsilon_{22}+0.0318$ \\
\hline
\end{tabular}

Regarding the behavior under in-plane shear, the parameter obtained trough a monotonic quasi-static test is the linear-elastic threshold limit for shear, or simply $S_{12_{y}}$. The procedure and its value are depicted in Figure 47.

Still concerning about shear response but under the cyclic loading case, experimental tests provides the evolution of the internal damage variable $d_{6}$ under pure shear state with relation to its associated thermodynamic associated force $Y_{6}$ applying the procedure described in Figure 25 and detailed in Eqs. (2.65), (2.67) and (2.73). Moreover, as highlighted by Ladevèze and LeDantec (LADEVEZE; LEDANTEC, 1992), it is important that in these tests the maximum number of 5 cycles be respected to avoid damage by the 
Figure 47: Linear-elastic shear threshold parameter obtaining.

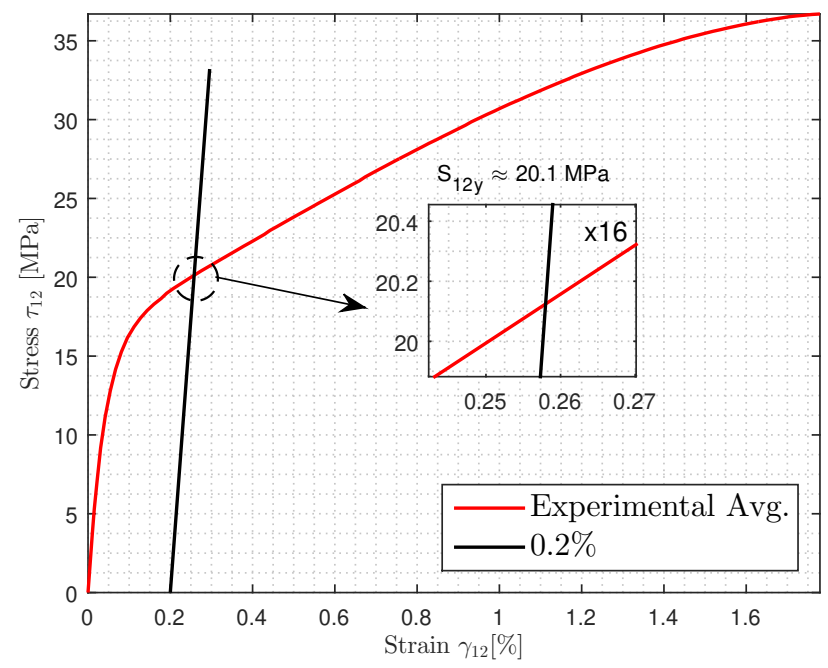

Source: by the author.

mechanism of low-cycle fatigue. Yet, since the cyclic tests are planned using the average of the monotonic ones, it is used the mean curves of the priors to evaluate the evolution of the internal damage variables regarding the increase of the respective thermodynamic force. For the internal damage variable $d_{2}$, under pure transverse tension stress state, the associated thermodynamic force is $Y_{2}$. In the same fashion, but now performing cyclic tests in $\left[90^{\circ}\right]_{8 T}$ laminates, the damage progression is studied. Figure 48 shows the obtained results whereas Table 12 sums up the obtained parameters.

Figure 48: Damage evolution for pure cases of in-plane shear (a) and transverse tension (b).

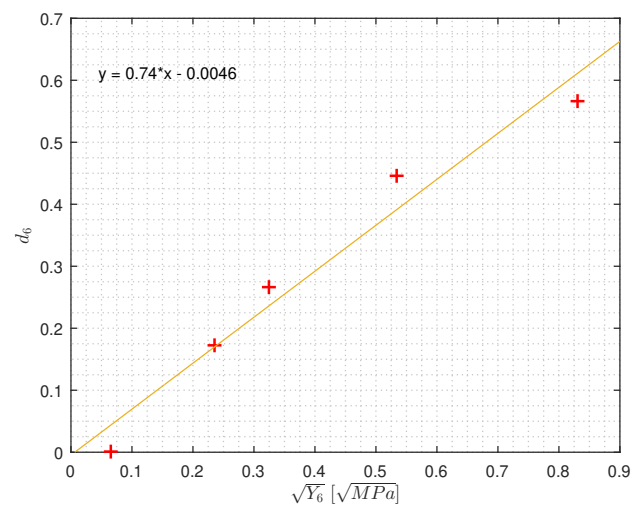

(a)

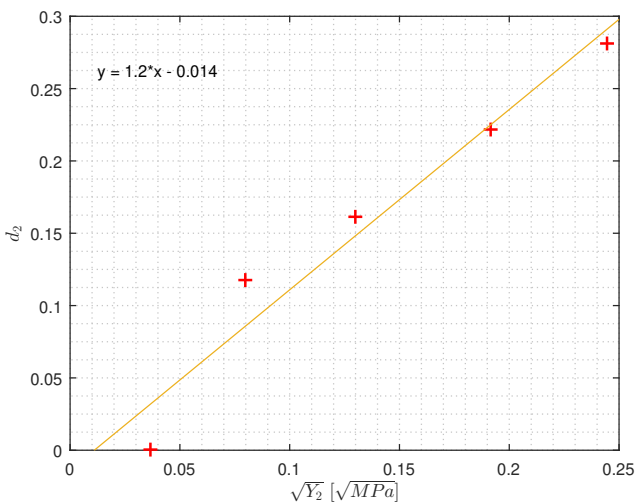

(b)

Source: by the author. 
Table 12: Damage evolution parameters for pure in-plane shear and transverse tension

\begin{tabular}{lcclc}
\hline Stress state & Parameter & Value & Description & Damage evolution law \\
\hline In-plane shear & $\sqrt{Y_{0}}$ & 0.00622 & Damage initiation & $d_{6}=0.74 \sqrt{Y_{6}}-0.0046$ \\
& $\sqrt{Y_{C}}$ & 1.35135 & Inverse angular coefficient & \\
Transverse tension & $\sqrt{Y_{0}^{\prime}}$ & 0.01167 & Damage initiation & $d_{2}=1.2 \sqrt{Y_{2}}-0.014$ \\
& $\sqrt{Y_{C}^{\prime}}$ & 0.83333 & Inverse angular coefficient & \\
\hline
\end{tabular}

Also, it is worth to mention that for each studied case, the number of specimens tested is of 3 .

According to Ladevèze (LADEVEZE; LEDANTEC, 1992), the thermodynamic force $\hat{Y}$ associated with the combined stress state is given by Eq. (2.70) following a linear combination between the shear $Y_{6}$ and transverse tension $Y_{2}$ associated thermodynamic forces and a coupling coefficient $b$. This parameter is there obtained as results of cyclic tests, that planned from the stress-strain levels achieved in quasi-static ones, in angle-ply specimens. Moreover, a good candidate is the $[ \pm 67.5]_{n s}$ laminate due to its good shear and transverse tension coupling. Starting from the average stress experienced by the laminate during the test, which is given as the ratio of the applied force and the initial area of the coupon, it is possible to obtain, using adequate transformation relations both the stress-strain states in material coordinates system. It follows, according to Herakovich (HERAKOVICH, 1998), that:

$$
\begin{aligned}
\sigma_{11} & =B \bar{\sigma}_{x}, \\
\sigma_{22} & =(1-B) \bar{\sigma}_{x}, \\
\tau_{12} & =-\frac{1}{2 m n}\left[B\left(1-2 m^{2}\right)+m^{2}\right] \bar{\sigma}_{x},
\end{aligned}
$$

where $m=\cos \theta, n=\sin \theta$ and,

$$
b=\left[\frac{m^{2}\left(2 m^{2}-1\right)+\frac{4 m^{2} n^{2} G_{12}}{E_{22}}\left(\frac{E_{22} \nu_{12}}{E_{11}}+1\right)}{\frac{4 m^{2} n^{2} G_{12}}{E_{22}}\left(\frac{E_{22}}{E_{11}}+\frac{2 E_{22} \nu_{12}}{E_{11}}+1\right)+\left(2 m^{2}-1\right)\left(m^{2}-n^{2}\right)}\right],
$$

and for the the strain state it follows,

$$
\begin{aligned}
& \varepsilon_{11}=m^{2} \varepsilon_{x}+n^{2} \varepsilon_{y}, \\
& \varepsilon_{22}=n^{2} \varepsilon_{x}+m^{2} \varepsilon_{y}, \\
& \gamma_{12}=-2 m n\left(\varepsilon_{x}-\varepsilon_{y}\right),
\end{aligned}
$$

in which $\varepsilon_{x}, \varepsilon_{y}$ are the global measured strains at the corresponding directions during the tests and considering $\varepsilon_{x y}=0$ for angle-ply laminates under tensile loads. Therefore, after performing the cylic tests, using the aforementioned relations it is possible to separate the 
influences of both shear and transverse tension by raising the $\tau_{12} \mathrm{x} \gamma_{12}$ and $\sigma_{22} \mathrm{x} \varepsilon_{22}$ curves. As derived by Ladevèze (LADEVEZE; LEDANTEC, 1992) and described by Herakovich (HERAKOVICH, 1998), using the definition of damage variable for shear, one can obtain the following relation

$$
b=\frac{\left(d_{6} \sqrt{Y_{C}}+\sqrt{Y_{0}}\right)^{2}-Y_{6}}{Y_{2}},
$$

where the parameters $Y_{C}$ and $Y_{0}$ are obtained in cyclic tests of $[ \pm 45]_{n s}$ angle-ply coupons. For such, it is only necessary to relate the damage variable $d_{6}$ value with the associated thermodynamic force $Y_{6}$ through a linear fit of the obtained points as shown in Figure 48a, for example. In total, five points are obtained, one for each cycle during the test. In turn, the thermodynamic forces and damage variables are obtained using Eqs. (2.65) and (2.67), respectively. For the sake of readability, these equations are repeated here.

$$
\begin{aligned}
Y_{2} & =\frac{\left\langle\sigma_{22}\right\rangle_{+}^{2}}{2 E_{22_{0}}\left(1-d_{2}\right)^{2}}, \\
Y_{6} & =\frac{\tau_{12}^{2}}{2 G_{12_{0}}\left(1-d_{6}\right)^{2}}, \\
d_{2} & =1-\frac{E_{22_{i}}}{E_{22_{0}}}, \\
d_{6} & =1-\frac{G_{12_{i}}}{G_{12_{0}}},
\end{aligned}
$$

in which $G_{12_{0}}$ is the shear modulus calculated as usual in quasi-static monotonic tests and $G_{12_{i}}$ is the same quantity but obtained in each cycle while for the transverse tension case it follows analogously. In this manner, using Eq. (3.17) and, consequently, knowing the value of the coupling coefficient $b$ in each cycle, it is possible to calculate $\hat{Y}$. Notice that since it is obtained one value of damage and thermodynamic force it follows that one value of $b$ is obtained for each cycle under analysis. However, in the methodology described, only one value of coupling is selected that, in the case, it corresponds to the one obtained in the cycle of greater damage and thermodynamic force values. Once determined, it is possible to relate the values of the damage variable $d_{2}$ with the total thermodynamic force $\hat{Y}$ and in a similar manner perform a linear fit in the data to obtain a relation of the type,

$$
d_{2}=c_{1} \sqrt{\hat{Y}}+c_{2}
$$

and, so, for $d_{2}=0$ or 1 it is determined $\sqrt{Y_{0}^{\prime}}$ and $\sqrt{Y_{C}^{\prime}}$, respectively. This occurs because the damage variable can be written as,

$$
d_{2}=\frac{\left\langle\sqrt{\hat{Y}}-\sqrt{Y_{0}^{\prime}}\right\rangle}{\sqrt{Y_{C}^{\prime}}} .
$$

Notice, thus, that is only the necessity of performing cyclic tests in \pm 45 and \pm 67.5 angle-ply coupons under longitudinal tension to determine the material model parameters 
associated, to know: $\sqrt{Y_{0}}, \sqrt{Y_{C}}, \sqrt{Y_{0}^{\prime}}, \sqrt{Y_{C}^{\prime}}$ and $b$. Herakovich (HERAKOVICH, 1998) still points out that the \pm 67.5 samples promotes transverse ultimate strains lower than those observed in $[90]_{n}$ specimens being more recommended to determine the transverse limits (stress and strain) since the $90^{\circ}$ laminates are susceptible to flaws (e.g. due to manufacture). Moreover, the $67.5^{\circ}$ coupon is of easy manufacturing and suffers low influence of interlaminar stresses in the failure mechanisms.

Some inconsistencies were verified while applying this calculation methodology to obtain the coupling parameter $b$ using as basis the cyclic tests data of Ribeiro (RIBEIRO, 2013). These arise due to the use of Eq. (3.17) that could lead to find any value for $b$, including negative ones that besides not having physical meaning also could not happen due to the very own construction of the coupling parameter introduced by Eq. (2.70). Therefore, the present author proposes an alternative to obtain this parameter that does not change the approach too much and is described in the sequence. The idea is to calculate $b$ only by using Eq. (2.70), that is: $\hat{Y}=Y_{6}+b Y_{2}$.

When performing a quasi-static monotonic test in the specimens of interest (i.e. one that generates coupling between shear and transverse tension) it is possible to:

- Plan the cylic tests;

- Obtain a apparent longitudinal modulus.

In its turn, during cyclic tests it is possible to verify the lost in stiffness through the evaluation of the apparent longitudinal modulus $E_{x}$ in each cycle and, consequently, the total verified damage. Analogously it follows that,

$$
d_{t}=1-\frac{E_{x_{i}}}{E_{x_{0}}}
$$

which $E_{x_{0}}$ is the obtained modulus in monotonic tests. Still, the total thermodynamic force in each cycle is given as,

$$
\hat{Y}=\frac{\bar{\sigma}_{x}^{2}}{2 E_{x_{0}}\left(1-d_{t}\right)^{2}}
$$

where $\bar{\sigma}_{x}$ is the average stress in the longitudinal direction verified during the tensile test. The thermodynamic forces $Y_{6}$ and $Y_{2}$ as well as the damage variables $d_{6}$ and $d_{2}$ are obtained as described by Ladevèze. However, in this case, the necessity of calculate $b$ based on the decoupled shear case is eliminated. Thus, isolating it in Eq. (2.70) it follows,

$$
b=\frac{\hat{Y}-Y_{6}}{Y_{2}},
$$

and it is obtained a value for $b$ in each cycle in the coupled case. Using Eq. (3.23) solves the issue of finding negative couple coefficients, what would indicate that the thermodynamic 
force associated with shear in the coupled case is bigger than the total thermodynamic force itself, what does not make any physical sense. Based on the previous arguments, monotonic tests followed by cyclic ones in off-axis $\left[45^{\circ}\right]_{6}$ and $\left[70^{\circ}\right]_{6}$ coupons are proposed aiming to verify the proposed calculation procedure for the coupling coefficient. Specimens geometry are based on the ASTM D3039 standard for transverse tension coupons possessing $175 \times 25 \times 1.25 \mathrm{~mm}$ as basis dimensions. The fiber orientations are chosen due to off-axis $45^{\circ}$ laminates possess maximum coupling between the interest damage mechanisms while as $70^{\circ}$ possess low coupling among them, having as main mechanism of damage the transverse tension, as depicted in Figure 49.

Figure 49: Percentage contribution to degradation of each stress nature of off-axis laminates as a function of the fiber orientation.

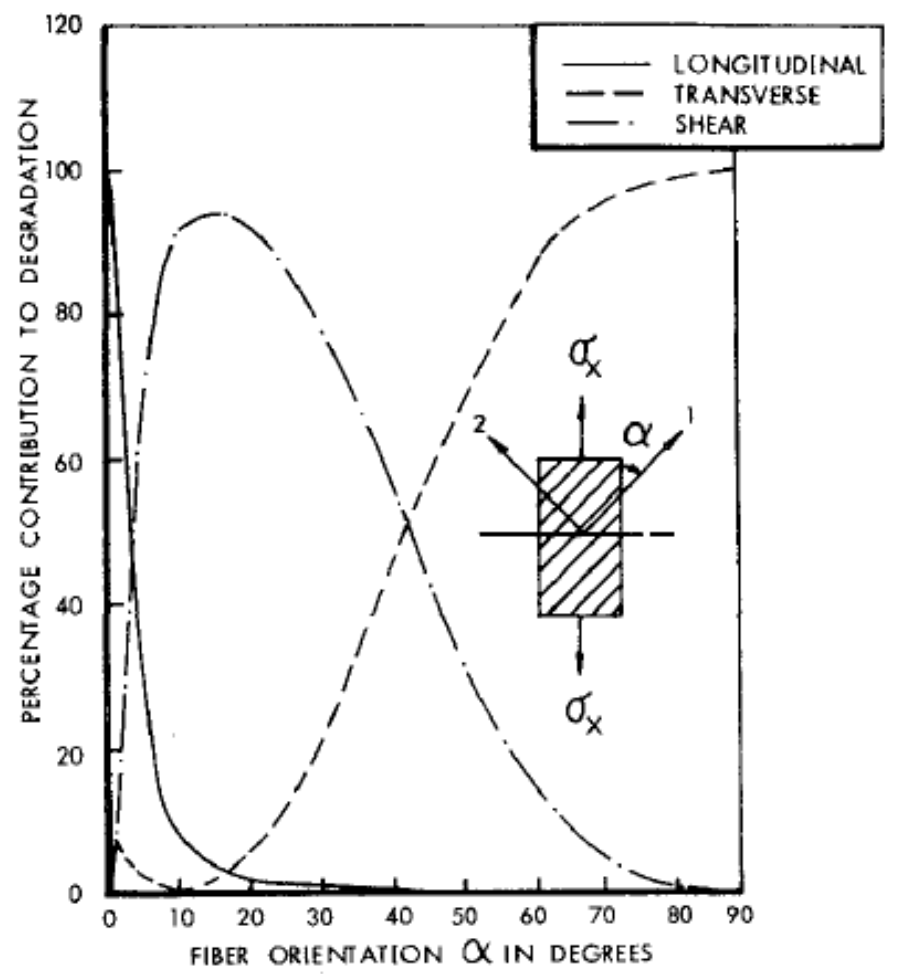

Source: after Sandhu (SANDHU, 1976).

Also, the choice for these stacks and geometry are based on a recommendation of Herakovich that for off-axis coupons the fibers should not go from one grip to the other of the testing machine. Testing velocity is based on Ribeiro (RIBEIRO, 2013) tests, which used $0.5 \mathrm{~mm} / \mathrm{min}$ for them. Moreover, due to high aspect ratio of the coupons $(\approx 15)$, it is satisfied that the global shear stress $\tau_{x y}$ is negligible. Therefore, only the global axial $\sigma_{x}$ stress is experienced by the laminate and used to obtain the stress and strain states in the material coordinate system using the CLT to account for the mutual influence of $\sigma_{22}$ and $\tau_{12}$. 
To perform the off-axis tests, six specimens are manufactured, being two for monotonic and four to cyclic testing. As a note, the average stress-strain curves monotonic tests are used to perform the cyclic ones. The overall results are depicted in Figure 50.

Figure 50: Monotonic and cyclic stress-strain curves of $\left[45^{\circ}\right]_{6}$ (a) and $\left[70^{\circ}\right]$ (b) off-axis coupons.

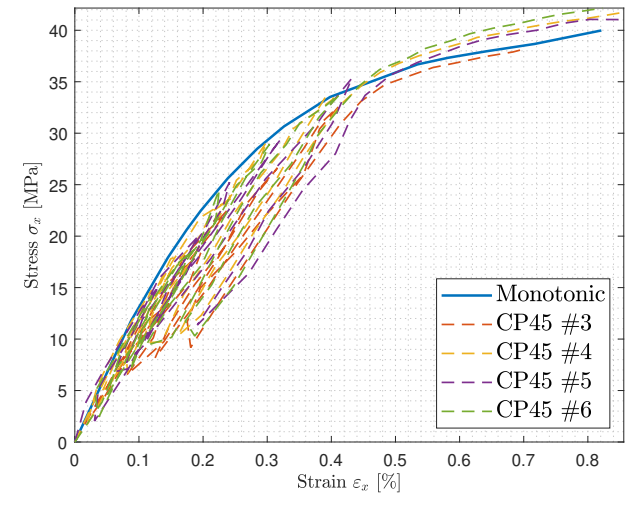

(a)

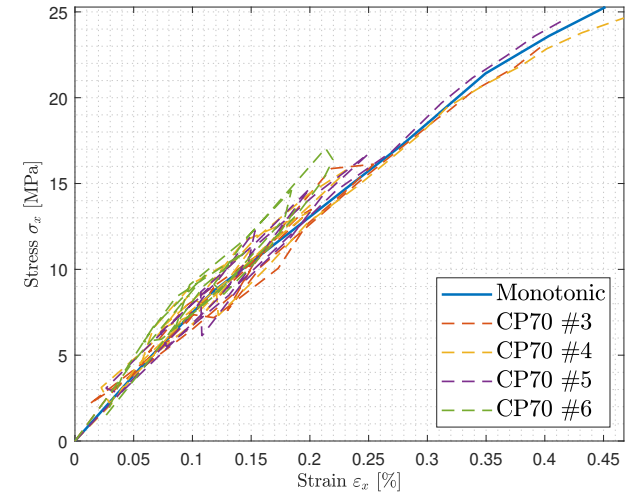

(b)

Source: by the author.

Notice that only the global $\varepsilon_{x}$ strain is depicted in Figures 50a and 50b. This is done for the sake of simplicity, since one needs to capture also $\varepsilon_{y}$ to assess shear and transverse tension effects in the material coordinate system, that is done using the DIC technique. The separate influences are depicted in Figure 51 for the $\left[45^{\circ}\right]_{6}$ off-axis laminate, as an example.

Figure 51: Transverse (a) and shear (b) stress-strain cyclic curves for $\left[45^{\circ}\right]_{6}$ coupons: material coordinate system.

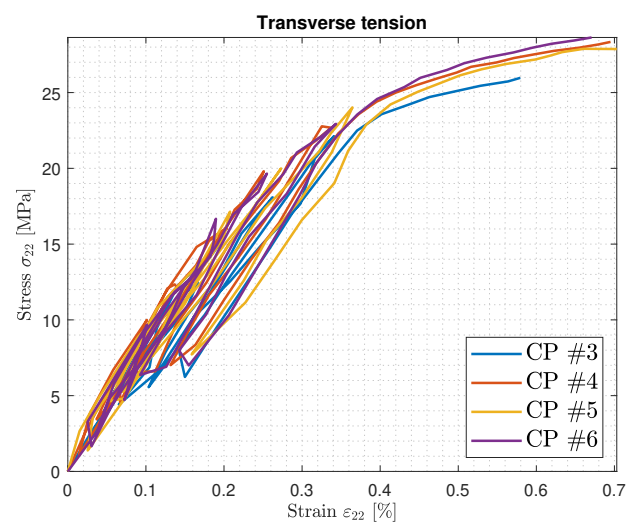

(a)

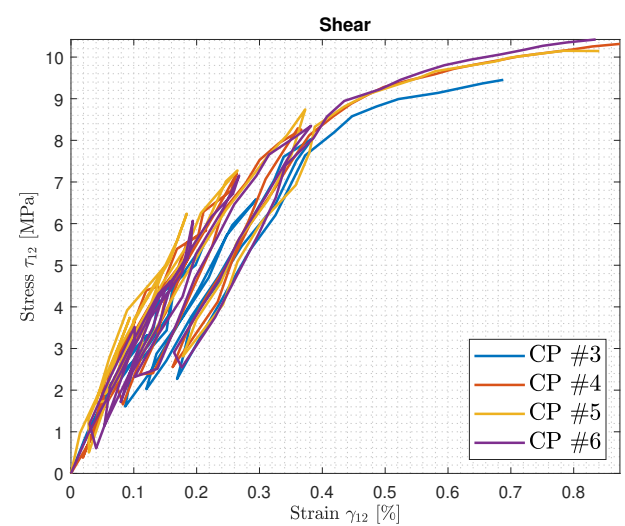

(b)

Source: by the author. 
It is worth to mention that the failure modes of all coupons are in agreement with the expected as shown in Figure 52.

Figure 52: Failure modes observed in off-axis $\left[70^{\circ}\right]_{6}$ (a) and $\left[45^{\circ}\right]_{6}$ (b) coupons.

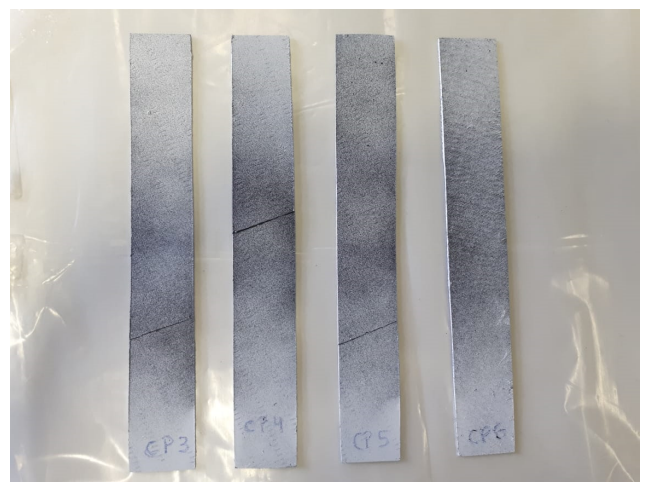

(a)

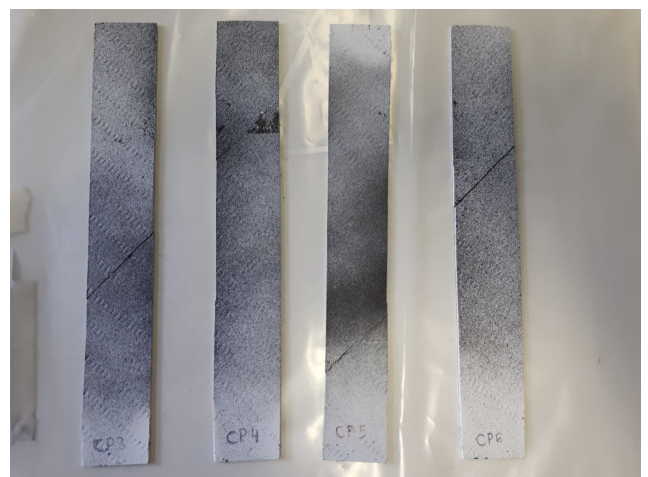

(b)

Source: by the author.

From the separate influences, it is now possible to obtain $d_{2}, d_{6} \times \sqrt{\hat{Y}}$ as usual and using Eq. (3.23) obtain the coupling coefficient $b$ for each cycle. Figure 53 shows the obtained results for damage evolution and in Figure 54 the coupling coefficient $b$ is depicted as a function of the total thermodynamic force $\sqrt{\hat{Y}}$ for both off-axis coupons in each cycle. Notice that in it, the values of $b$ for both orientation angles of $45^{\circ}$ and $70^{\circ}$ are the average ones of all the tested specimens for each cycle as listed in Table 13.

Table 13: Coupling coefficient, thermodynamic force and damage variables values results for off-axis laminates (average of tested coupons)

\begin{tabular}{ccccc|cccc}
\hline Coupon & \multicolumn{5}{c|}{$[45]_{6}$} & \multicolumn{4}{c}{$[70]_{6}$} \\
\hline Cycle & $d_{2}$ & $d_{6}$ & $\sqrt{\hat{Y}}$ & Coupling $b$ & $d_{2}$ & $d_{6}$ & $\sqrt{\hat{Y}}$ & Coupling $b$ \\
\hline 1 & 0 & 0 & 2.972 & 1.379 & 0 & 0 & 2.146 & 1.306 \\
2 & 0.109 & 0.068 & 4.278 & 1.387 & 0.082 & 0.078 & 3.178 & 1.565 \\
3 & 0.167 & 0.129 & 5.906 & 1.384 & 0.166 & 0.143 & 4.389 & 1.626 \\
4 & 0.238 & 0.262 & 7.812 & 1.381 & 0.195 & 0.164 & 5.269 & 1.599 \\
5 & 0.311 & 0.341 & 10.188 & 1.381 & 0.318 & 0.307 & 9.301 & 1.615 \\
\hline
\end{tabular}

Some interesting features of Figure 54 are noteworthy. Firstly, it is noticeable that the value of the coupling coefficient $b$ fluctuates more during the tests for the $70^{\circ}$ specimen, which is the one with the transverse stress dominated degradation response. Also, it is perceived that $b$ grows in the first three cycles and then stabilizes for the last two ones. 
Figure 53: Damage evolution: coupled cases of transverse tension (a) in-plane shear (b).

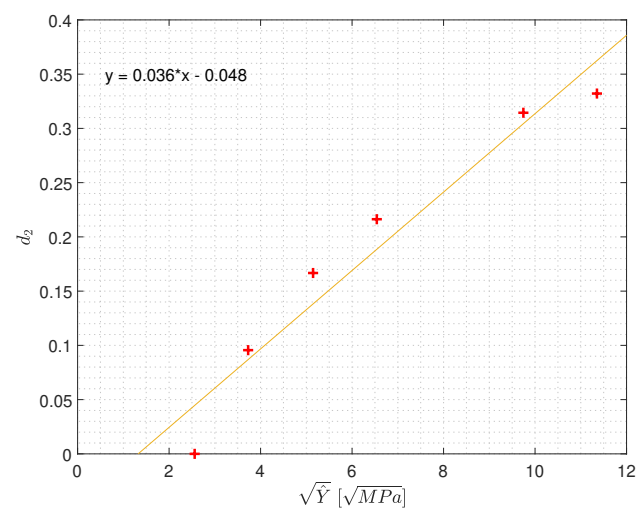

(a)

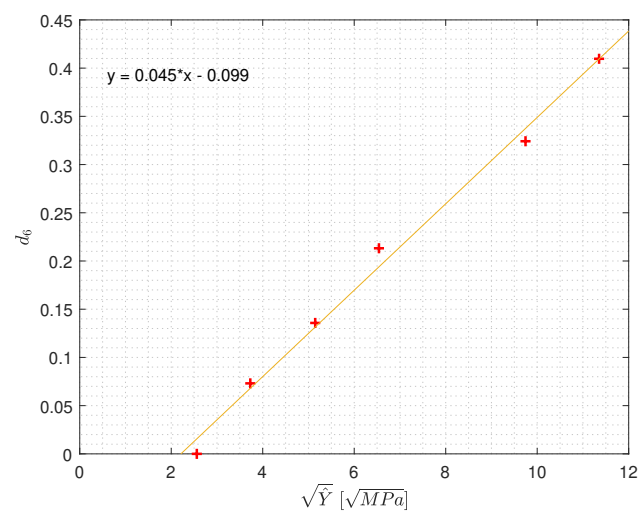

(b)

Source: by the author.

Figure 54: Coupling coefficient $b$ as a function of the equivalent thermodynamic force.

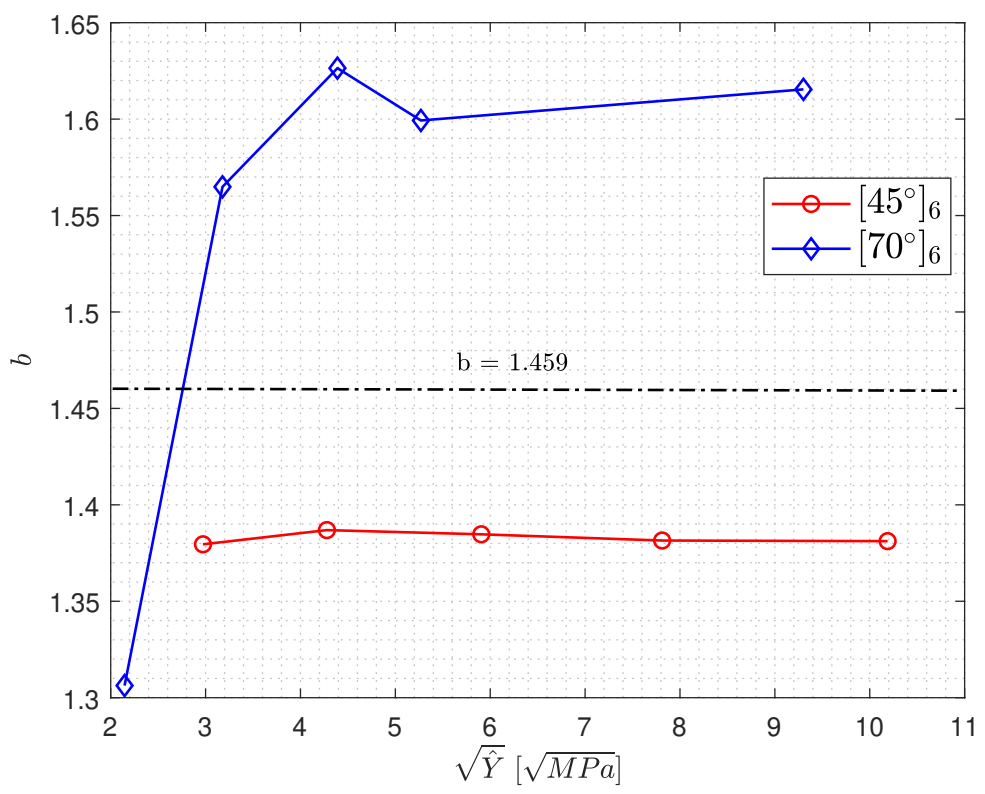

Source: by the author.

Regarding the $45^{\circ}$ laminates behavior, it is clear to see that the obtained values of $b$ are almost constant during the tests. Thus, for the maximum possible simultaneous influence of both transverse and shear stresses in degradation, the coefficient that translates this feature seems to not vary significantly. Lastly, it is verified experimentally that the coupling coefficient $b$ is a function not only of the thermodynamic force level but, also, varies with the fiber orientation. As an example, in a naive preliminary proposal, one could take the average values of $b$ for each orientation and raise the data shown in Figure 55. 
Figure 55: Coupling coefficient $b$ as a function of the fiber orientation $\theta$.

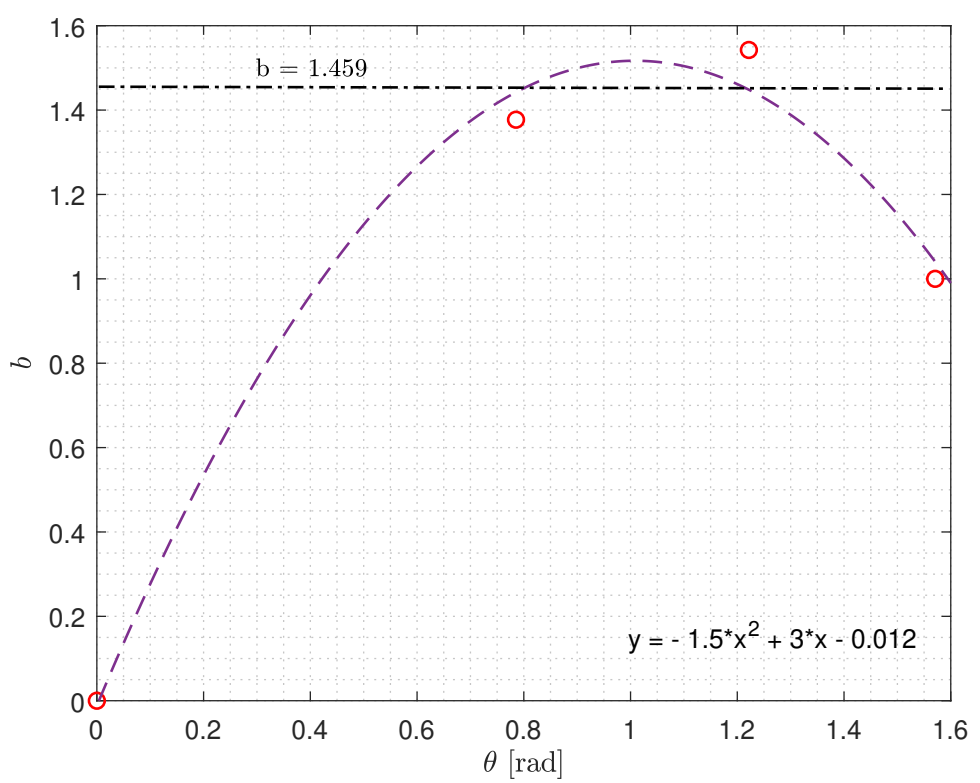

Source: by the author.

In the example, a quadratic fitting of the data is chosen. Notice that even though no experimental values of $b$ where obtained for $0^{\circ}$ and $90^{\circ}$ it is trivial that it must assume values of 0 and 1 , respectively.

The discussion about the parameter $b$ was performed to emphasize some key aspects and issues that can arise while trying to obtain it and to raise up some future work proposals. Nevertheless, due to the selected material model formulation, a constant value of $b$ needs to be obtained. Thus, it is simply taken as the average value of the average values obtained for $b$ for each specimen in each cycle. Finally, it follows that,

$$
b=1.459 \text {. }
$$

\subsection{Methodology for Shear-After-Impact}

In this section, the experimental apparatus for SAI testing is described. Firstly, shear testing using the three rail fixture described in the ASTM D4255 standard is introduced as a tool for damage tolerance evaluation. Also, the drop-weight specimen from the ASTM D7136 standard is adapted so it can be tested in the rail apparatus. Finally, the computational-experimental methodology for SAI is proposed.

\subsubsection{Rail Test}

Based in the extensive literature of shear testing for composite materials, it can be observed that several procedures of obtaining shear properties for these materials currently 
exists. One of the consolidated references for performing these tests is the ASTM D4255 standard for rail test. In it, two procedures are introduced with this goal as shown in Figure 56 .

Figure 56: Two rail (a) and three rail (b) test standardized apparatus.

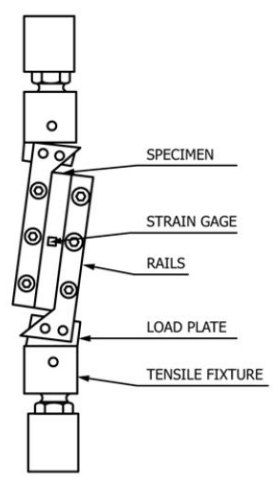

(a)

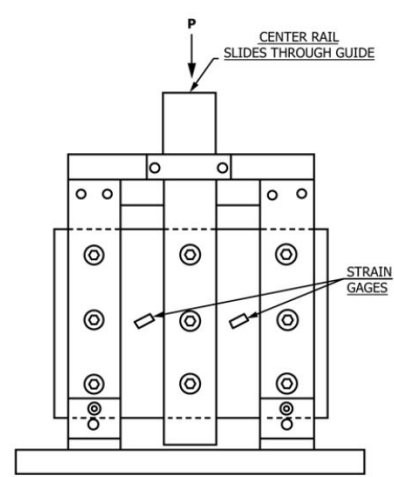

(b)

Source: American Society for Testing and Materials.

In Procedure A (Fig. 56a), two steel rails are screwed for fixing the specimen and the applied tensile load induces a shear state in the coupon. On the other hand, in Procedure B (Fig. 56b) three rails are screwed with the same purpose. The main difference is that the center rail is free to slide through a guide while the side rails are fixed to a support base. A compression load is applied in the central rail which induces a shear state in the composite laminate. The shear stress in both procedures is obtained by,

$$
\begin{aligned}
\tau_{i} & =\frac{P_{i}}{A} ; \text { for } 2 \text {-rail, } \\
\tau_{i} & =\frac{P_{i}}{2 A} ; \text { for } 3 \text {-rail, }
\end{aligned}
$$

where $P_{i}$ is the applied load at the $i$-th data point. Also, the ultimate shear strength is given as,

$$
\begin{aligned}
F^{u} & =\frac{P^{\max }}{A} ; \text { for } 2 \text {-rail, } \\
F^{u} & =\frac{P^{\max }}{2 A} ; \text { for } 3 \text {-rail, }
\end{aligned}
$$

in which $F^{u}$ is the ultimate shear stress and $P^{\max }$ is the force carried out by a coupon that is the lesser between one of the following:

1. Maximum force before failure;

2. Force at 5\% engineering shear strain; 
3. Force at the bending limit.

The last is defined by,

$$
B_{y}=\frac{\left|\varepsilon_{1}-\varepsilon_{2}\right|}{\left|\varepsilon_{1}+\varepsilon_{2}\right|} \times 100 \leq 10 \%
$$

with $B_{y}$ being the bending limit and $\varepsilon_{1,2}$ the measured strains between opposite faces of the coupon. The $B_{y}$ value indicates the difference among stress-strain or force-strain slopes from opposite sides of the specimen and its limit is defined as $10 \%$ by the standard.

Lastly, the engineering shear strain is calculated as,

$$
\gamma_{i}=\left|\varepsilon_{(+45)}\right|+\left|\varepsilon_{(-45)}\right|
$$

where $\varepsilon_{(+,-45)}$ are the indicated normal strains in the $+45^{\circ},-45^{\circ}$ directions at the $i$-th data point and $\gamma_{i}$ is the engineering shear strain.

In this work, the three rail apparatus for shear tests will be employed for the SAI analyses due to dimensional adaptations made in the drop-weight coupons for accommodating in the rails.

\subsubsection{Drop-Weight Specimen Adaptation}

The complete drop-weight system is depicted in Figure 57. The test specimen is fixed with the toggle clamps that are attached to the inertial base. There is a support used to attach the impactor with the Kistler 9011A load cell and to set the test height. This load cell is a piezoelectric transducer capable to measure compression forces at the $z$-axis (same direction of gravitational acceleration) under dynamic or quasi-static conditions. Thus, some quartz crystals generate an electric charge proportional to the mechanic loading with a sensitivity of $4.3 \mathrm{pC} / \mathrm{N}$ and a range of $96 \mathrm{kN}$. For the displacement measurement a M70LL/50 Laser Distance Sensor (LDS) from MEL Intelligent Sensors \& Measuring Systems is used. With a measuring range of $50 \mathrm{~mm}$, sampling rate of $400 \mathrm{kHz}$ and $100 \mathrm{kHz}$ of measuring frequency it is capable of capturing with high precision the displacements occurring in an impact event during a short amount of time. Both the LDS and load cell are connected to a charge amplifier/data acquisition unit from Kistler named LabAmp (model $5165 \mathrm{~A}$ ). This equipment can be used wherever dynamics signals of mechanical quantities are measured with piezoelectric, piezotron (IEPE) or voltage output sensor (for example, the LDS from MEL). The data acquisition unit is capable of capturing up to $200 \mathrm{kSps}$ (200,000 samples per second) in each of its four channels simultaneously, integrated with digital low-pass, high-pass and notch filters if needed. Moreover, the software can capture data and make its fast Fourier transform (FFT) in real time being a powerful equipment for the experiments made by the Group of Aeronautical Structures (GEA - Grupo de Estruturas Aeronáuticas, from Brazilian Portuguese). Finally, it is also possible to index 
some weights to the support with the objective to increase the impact energy for some test. The drop-weight system, or simply, drop-tower, equipped with the aforementioned is capable of measuring the force, displacement and energy histories (i.e. force, displacement and energy through time).

Figure 57: Drop-weight test complete system.

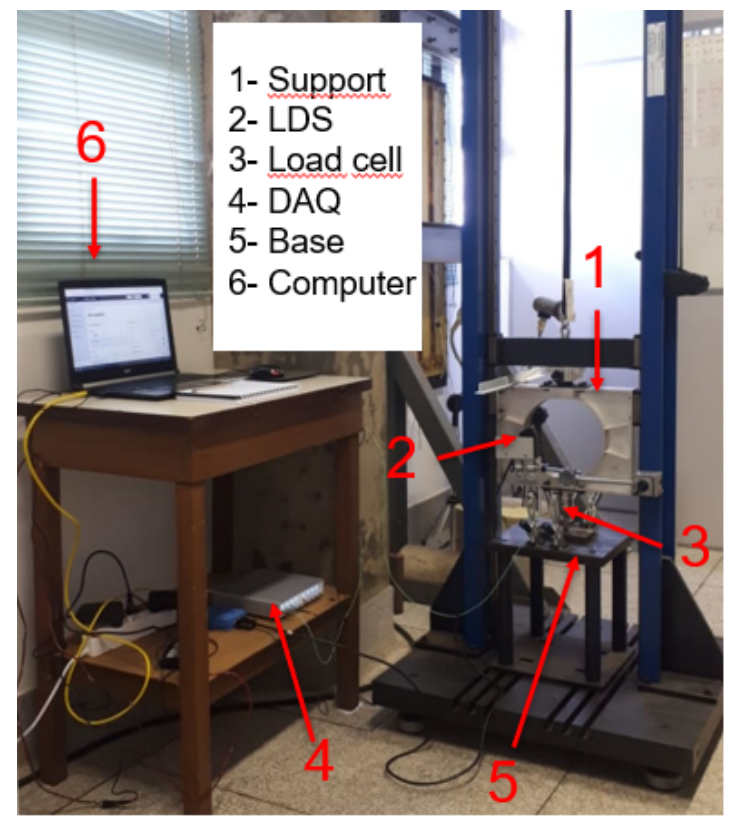

Source: by the author.

Aiming to perform experimental shear-after-impact tests, the 3-rail apparatus described in the aforementioned ASTM standard was manufactured and is depicted in Figure 58.

Figure 58: Three rail apparatus for shear and SAI tests.

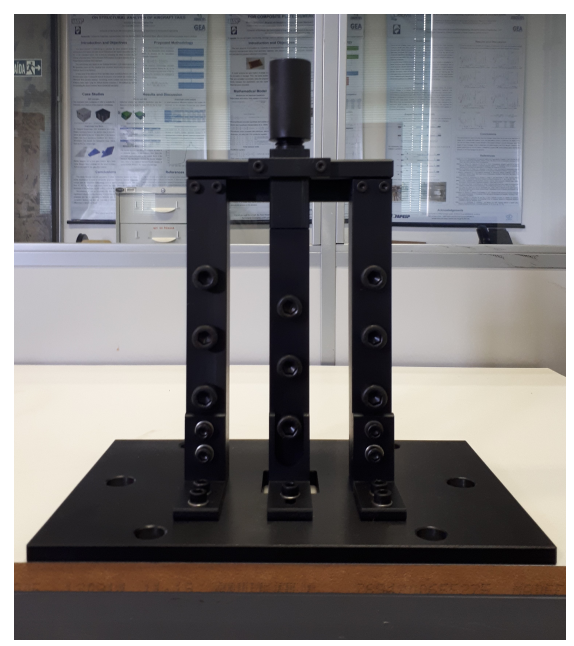

Source: by the author. 
Firstly, some care was taken to guarantee good performance of the rails. After manufacture (which followed the adequate tolerances), the set went through a black oxide process to protect the material from rust maximizing its corrosion resistance. Also, seasonal adequate lubrication of the testing fixture is done as an additional caution to prevent friction of the center rail with the guiding hole.

The next step is to adapt the drop-weight coupon so it can be also used in the 3 -rail test. This occurs because of the presence of some guiding pins in the drop-tower basis (Figure 59).

Figure 59: Drop-tower basis with guiding pins (circled).

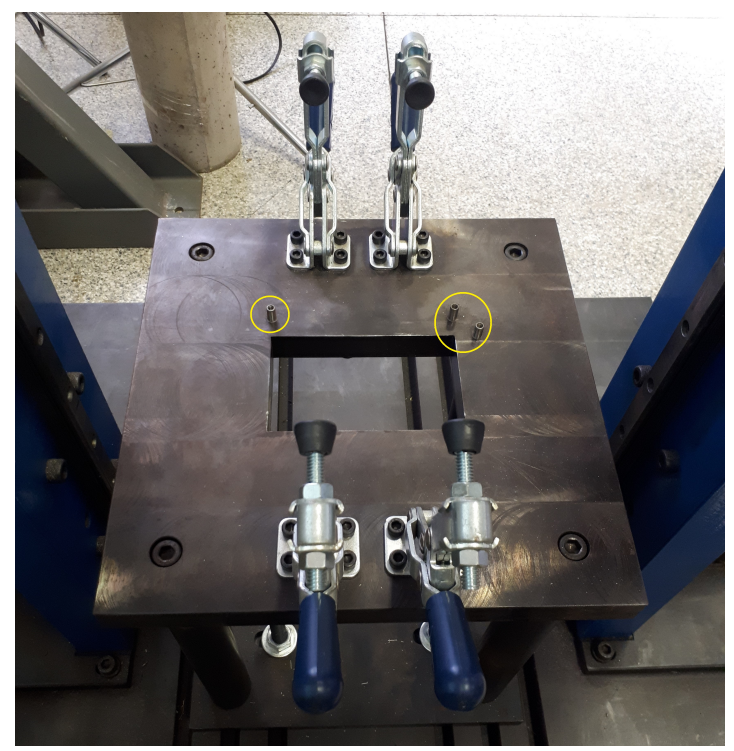

Source: by the author.

As can be noticed, these pins restricts the specimen movement during impact tests. Besides this, it also ties the specimen size, since impact should be performed at the center of it. Thus, a new specimen is proposed to perform the drop-weight test in these conditions and it is thought for further post-impact shear testing. The specimen scheme is shown in Figure 60.

The SAI specimen is a square plate that can be impacted in two points represented by the crosses. The dashed lines represents the limits of the drop-weight specimen described in the ASTM D7136 standard. As can be noticed, the impact points for SAI coincides with the impact points of two overlapping drop-test coupons. Thus, this specimen is positioned in the drop-weight basis as usual and fixed by the four clamps. An impact test is performed and, after it, the laminate is turned upside-down and is rotated so that another impact event can be performed. By following this procedure, two impact tests are conducted and the impacted side of one is the non-impacted side of the other; but these are not at the same point, as represented in the scheme in Figure 61. 
Figure 60: Proposed specimen for SAI testing.
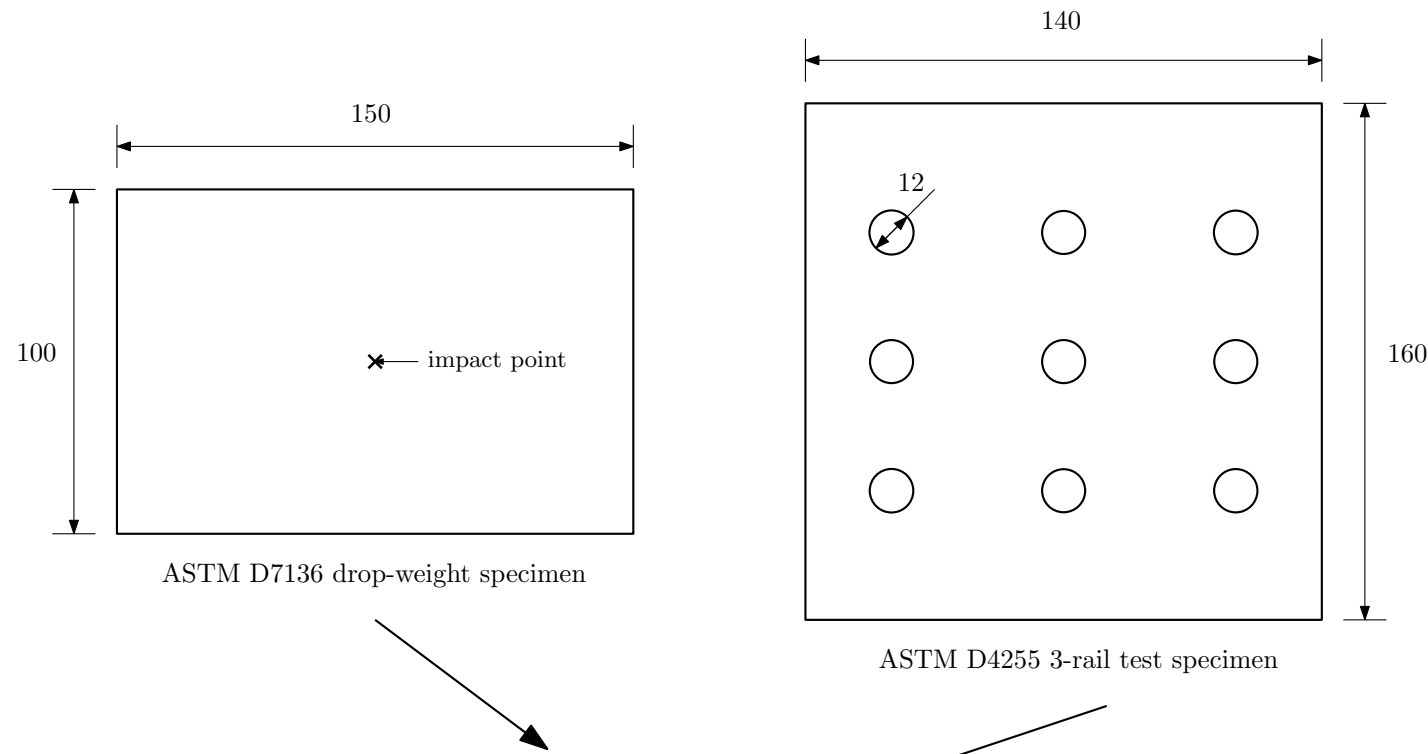

ASTM D4255 3-rail test specimen

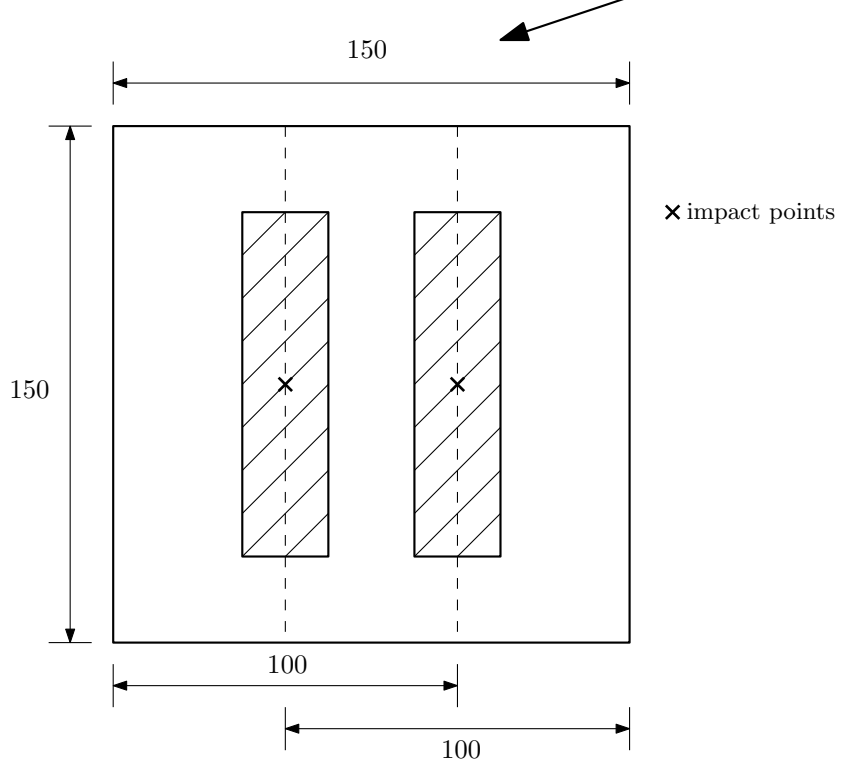

Specimen for SAI

Source: by the author.

Since impact is a non-uniform event, there are no guarantees that symmetrical damage conditions will occur during the impact events and, furthermore, equilibrium of force and momentum during the SAI testing is not assured although, for LEI at the BVID limit, it can maybe be a reasonable approximation taking it into account.

Going back to the SAI specimen description, in Figure 60 the hatched regions coincides with the free areas (i.e. visible ones) of the rail test coupons. So, these are the areas in which the DIC technique will be carried out. Moreover, after performing both impacts, the specimen holes (for fixture of it in the shear test apparatus) are made and SAI test is done. It is worth to mention that this free region is very small possessing only 
Figure 61: Impact events scheme in the SAI proposed coupon.

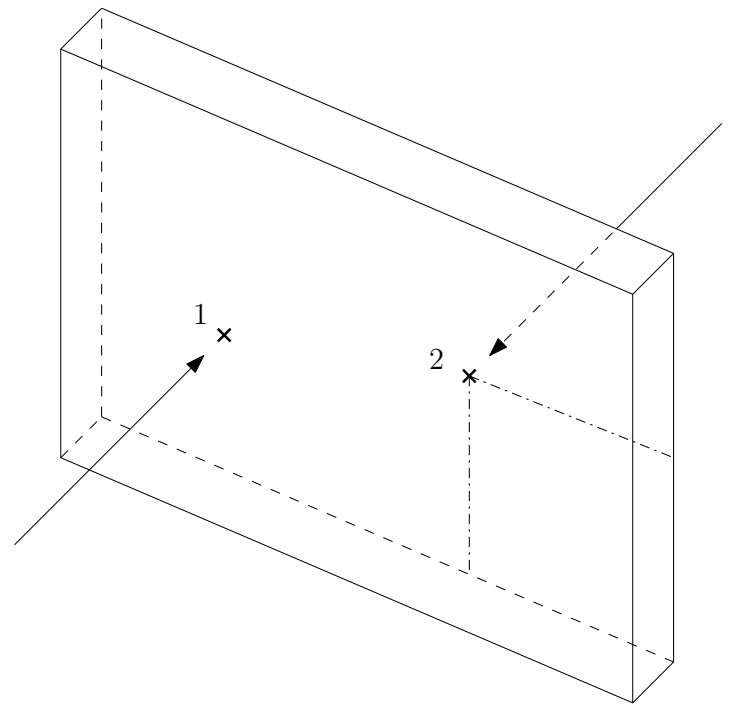

Source: by the author.

$24 \mathrm{~mm}$ of width. Therefore, it is of fundamental importance to assure that the impacts will be low-energy ones with localized damage, reinforcing the significance of the adopted strategies for its characterization, namely: mode one energy ratio (MOER) and loss factor criteria.

Figure 62 presents the adopted strategy scheme. Firstly, the coupon concept is thought so it can be tested under drop-weight impact and 3-rail shear experiments. Provided with the composite laminate stiffness parameters (which allows the calculation of $D^{*}$ ), dimensions (allows obtaining $K_{b s}$ ) and elastic properties (making possible to obtain $K_{\alpha}$ ) the loss-factor limit condition can be verified for the intended impact test. If it is satisfied, the drop-weight test is realized and its results provides conditions for computing the energy associated with the first eigenmode. Thus, if this energy is at least equal to $98 \%$ of the total impact energy, than the MOER criterion is also satisfied, validating the drop-weight test since it can be classified as a low-energy impact event. Finally, provided with the drop-test results, the impacted coupon is used for further SAI experiments and, also, computational model feeding is carried out.

Notice that the loss factor criterion can be satisfied and, even though, if the MOER criterion is not, the specimen has to be rethought. Moreover, the usage of the first is only to get a previous knowledge of the damage extension and laminate behavior at the impact event. This emphasizes that the loss factor approach is only an approximation for experimental drop-weight test concept. The true factor which defines the impact event as a low-energy one is the MOER criterion. Nevertheless, due to the reduced impact areas of the proposed SAI specimen, very low heights are going to be used for the forthcoming drop-tests and, consequently, both localized damage (characterizing a quasi-static response 
Figure 62: Proposed low-energy impact evaluation strategy.

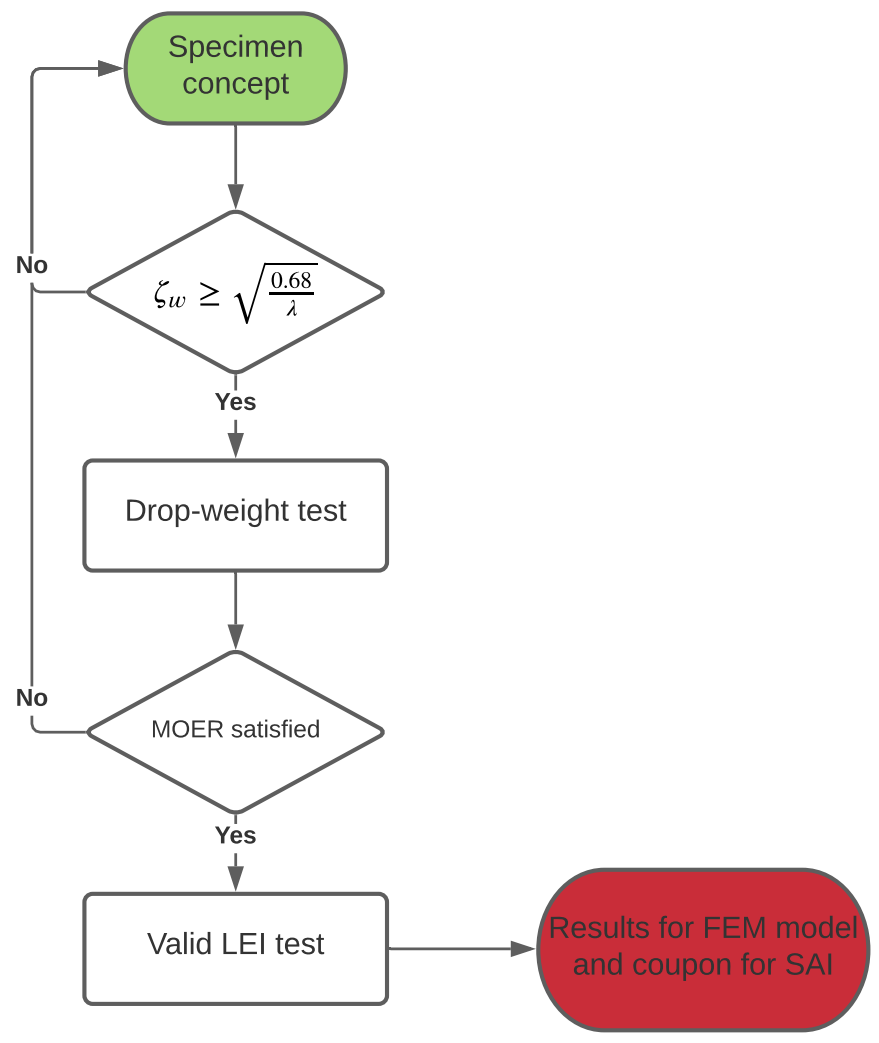

Source: by the author.

of the laminate) and low-energy regime are expected to occur.

\subsubsection{Proposed Methodology for SAI}

As far as the author knows, there are no documented works with the objective of proposing a methodology or guidelines for shear-after-impact damage, resistance, strength and/or behavior assessment in the literature. The present author found only a series of two works by Feng et al. (FENG et al., 2017a; FENG et al., 2017b) in which the buckling, post-buckling and fatigue performances of stiffened composite laminated panels with different impact positions under in-plane shear-after-impact loading is investigated. In these, impacts of 50J energy levels were introduced in the stiffened panels and further SAI experiments were carried out. Comparison with undamaged panels is done and the changes in buckling and failure loads are obtained.

Thus, the present work arises with the objective of fulfill the existent gap in the literature. Provided with the introduced barely-visible impact damage natural of low-energy impact events and the novel specimen thought especially for shear-after-impact testing, a methodology for SAI analysis is proposed. Figure 63 shows up a schematic flowchart of 
the proposed procedure.

Figure 63: Proposed methodology flowchart for SAI analysis.

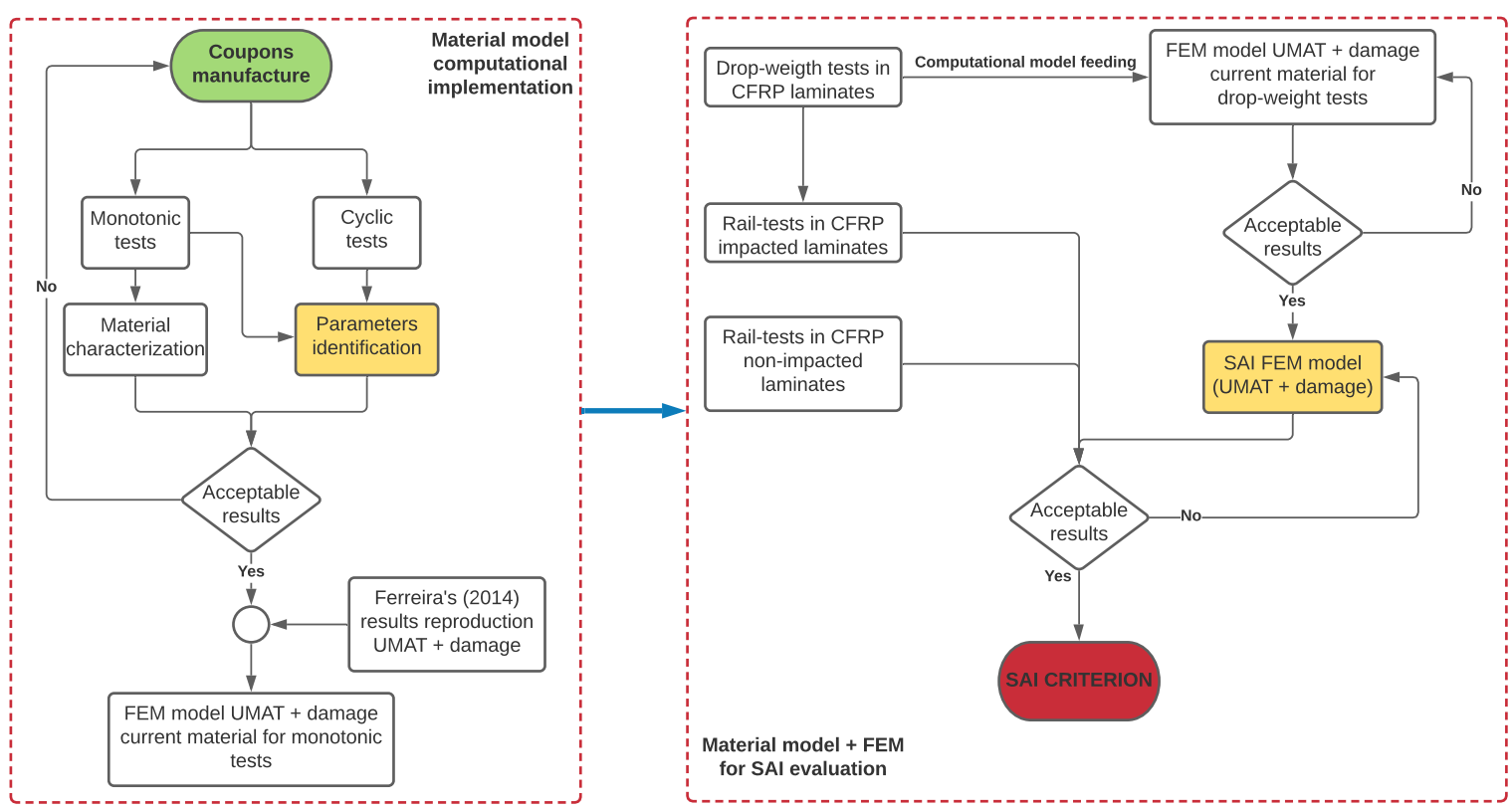

Source: by the author.

The SAI methodology is based in a computational-experimental approach. In it, drop-weight tests are performed in metallic plates to serve as input and confrontation experimental data with finite element simulations in ABAQUS. If these presents good agreement, than a mesh convergence analysis is performed. Next, since the Boeing fixture does not provides a "trivial condition of fixture", a study of the influence of the boundary conditions on the computational model needs to be realized. These results will feed the simulations using the employed material model for composites and UMAT subroutines. In parallel, coupons manufacture for monotonic and cyclic quasi-static tests shall be performed so material characterization and the material model parameters identification for the employed CFRP system are assessed. Of course, the SAI coupons are also manufactured for further experiments. If acceptable results during the experiments are achieved, than the FEM model for the current material is fed. Reproduction of Ferreira's (FERREIRA, 2014) results for implicit simulations in ABAQUS using UMAT and damage evolution are done to serve as basis for verification of the FEM model of the impact simulations to be performed with the employed material system. Provided with all of these, and taking care to follow the procedure for LEI analysis explained in Figure 62, drop-weight tests are performed in the CFRP laminates and these will serve for both computational model feeding and rail-tests in these damaged coupons. The last is used to evaluate the potentialities of the employed material model and strategy. Also, rail tests in undamaged CFRP laminates are carried out and are used as a sound basis for comparison with those realized in 
the damaged plates. Finally, with the collected computational and experimental results, the shear-after-impact adopted methodology for evaluation of the behavior of composite laminated plates submitted to post-impact shear loads is evaluated. If satisfactory results were obtained, than a SAI criterion based on the damage index concept introduced in the work by Medeiros (MEDEIROS, 2016) can maybe be proposed.

Moreover, due to the dimensions of the proposed SAI specimen, some interesting tests can be performed. Regarding to Figure 60, different impact energies can be used to induce damage in the impact points 1 and 2. Obviously, this intentionally introduces unbalanced testing conditions. Nevertheless, it also creates the possibility to evaluate the influence of this effect on the composite laminate damage evolution and failure behavior. Another possible test is to induce damage only in one of the impact points. Thus, the peculiarities in the response of the damaged an undamaged regions during the rail test can be simultaneously studied. Figure 64 shows up a summary of the SAI analysis.

Figure 64: Summary of SAI analysis.

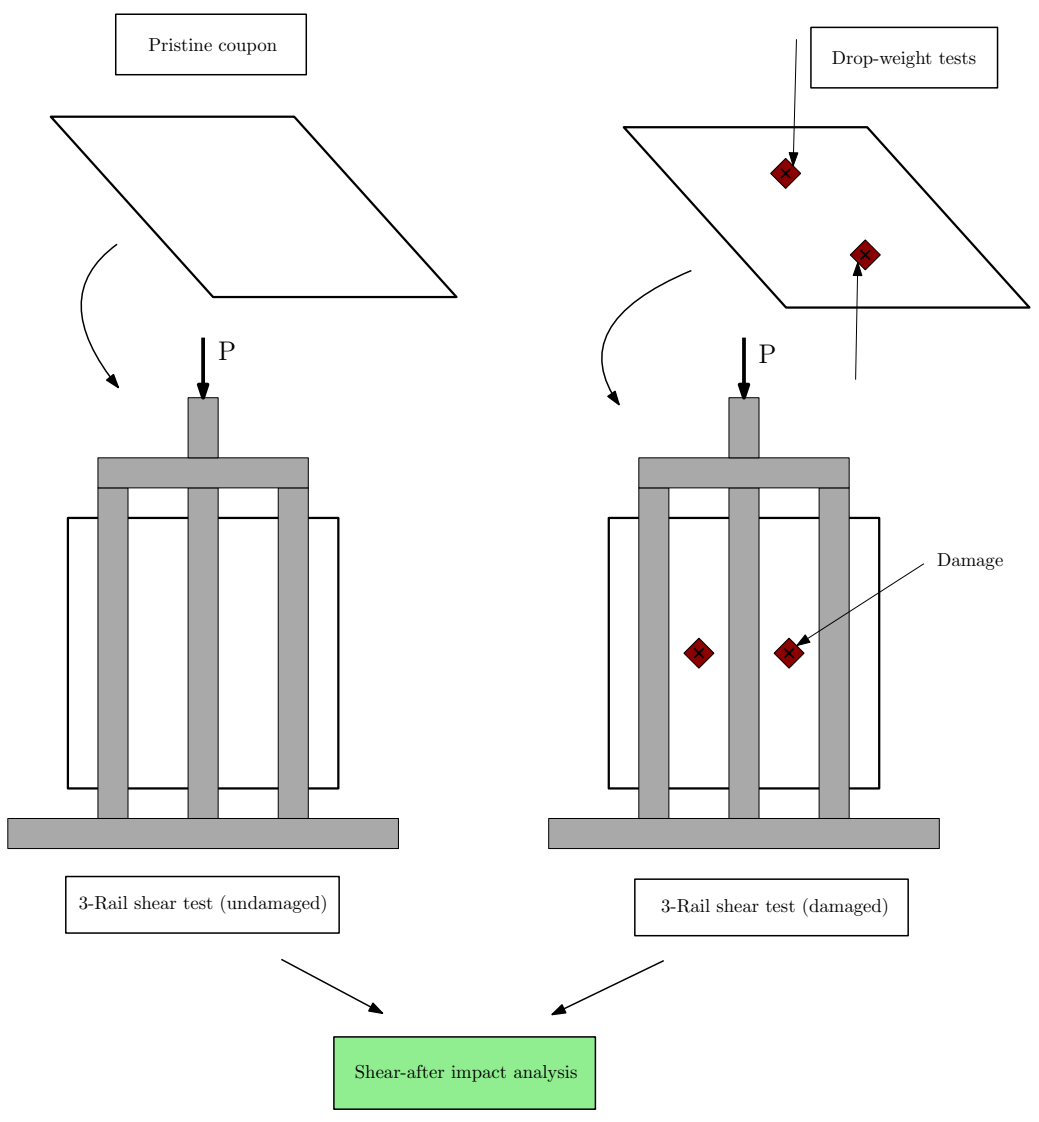

Source: by the author.

Lastly, is important to highlight that the rail test produces a simple shear stress state in the laminate, as discussed in Figure 4. 


\subsubsection{SAI Damage Index}

In order to access residual strength and, consequently, damage tolerance of structures it is common to define metrics that are used to quantify the damage severity and to provide insight about the necessity of repairing (or even discarding) these structures using structural health monitoring devices and techniques. In the case of composite laminates it is usually more difficult to define one metric that incorporates all variables that are relevant to the problem since it depends, for example, of the fiber orientation. Now, since the SAI analysis is basically based in the comparison between the pristine and damaged laminates shear stress-strain curves, a good strategy is to define a damage index that is energy-based, thus a phenomenological one. It follows,

$$
\mathrm{DI}_{\mathrm{SAI}}=1-\frac{\bar{E}_{D}}{\bar{E}_{P}},
$$

in which,

$$
\begin{aligned}
& \bar{E}_{D}=E_{D}-E_{D}^{\min }, \\
& \bar{E}_{P}=E_{P}-E_{D}^{\min },
\end{aligned}
$$

where $E_{D}$ and $E_{P}$ are the toughness of the damaged and pristine specimens, respectively. Notice that the damage index for SAI ranges from 0 (no damage) to 1 (fully damaged) with the parameters $\bar{E}_{D}$ and $\bar{E}_{P}$ defined such that this is always true.

Furthermore, when designing structures it is of good practice to define a stress allowable. In the case, following the strategy of Medeiros (MEDEIROS, 2016) that is based on the factor of safety FS $=1.5$ defined by the U.S. Federal Aviation Administration (FAA) for aeronautic structures, here it is defined that the allowable toughness for damaged specimens, which is related to the ultimate shear stress of the pristine coupon is such that,

$$
E_{D}^{\min }=\int_{0}^{\gamma_{12}^{\text {frac }}} \frac{\tau_{12}^{\mathrm{ult}}}{\mathrm{FS}} d \gamma
$$

where $\gamma_{12}^{\text {frac }}$ is the fracture shear strain and $\tau_{12}^{\text {ult }}$ is the ultimate shear stress for the undamaged coupon. Therefore, any laminate submitted to a low-energy impact which possess a toughness greater than that defined by Eq. (3.31) is within the range $0 \leq \mathrm{DI}_{\mathrm{SAI}} \leq 1$. Of course, if $E_{D}=E_{D}^{\text {min }}$ than $\mathrm{DI}_{\mathrm{SAI}}=1$. Figure 65 exemplifies the above-mentioned.

Therefore, the damage index assessment follows a simple procedure. Firstly, one needs to submit a pristine coupon under 3-rail test to obtain the stress-strain curve and integrate it. From the ultimate shear stress the minimum allowable toughness is obtained. Than, after performing drop-weight tests to introduce damage, the 3-rail test is conducted in the impacted coupon. After that, the stress-strain curve is obtained and, consequently, the toughness for the damaged specimen. If it is between the range $E_{D}^{\min } \leq E_{D} \leq E_{P}$, than the damage index $\mathrm{DI}_{\mathrm{SAI}}$ is calculated using Eq. (3.29) and, with it, the damage severity is obtained. 
Figure 65: SAI damage index: example of experimental curve defining $E_{P}$ and $E_{D}^{\min }$.

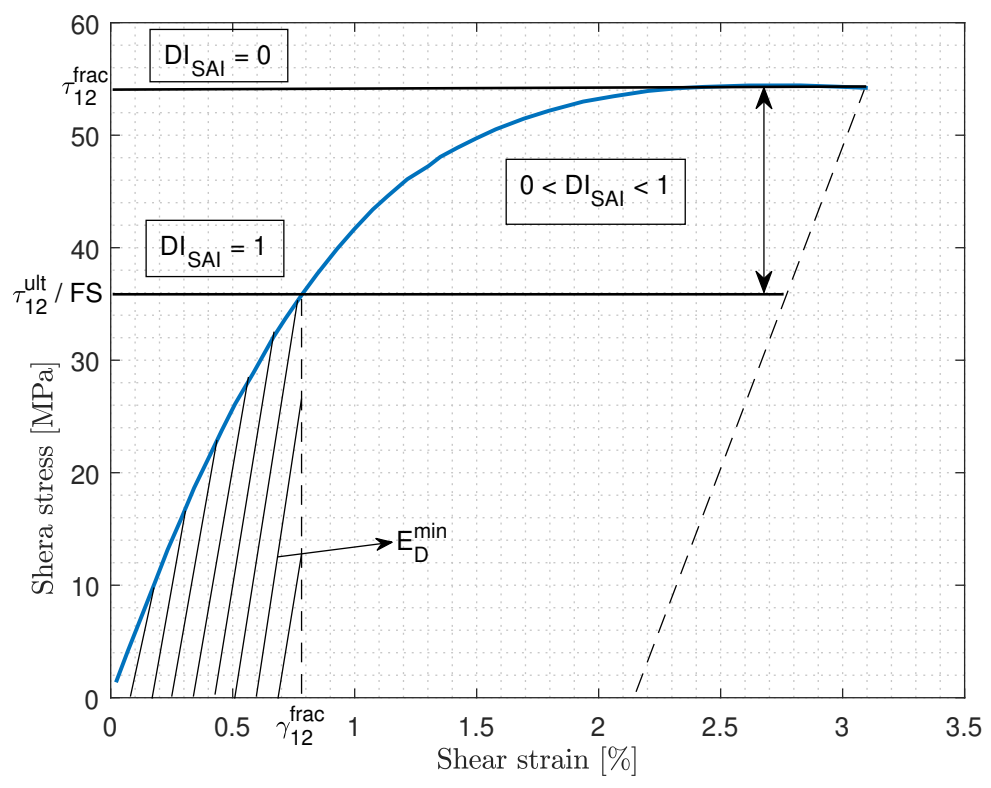

Source: by the author. 



\section{APPLICATION OF THE METHODOLOGY}

This chapter presents the experimental methodology for assessing the shear-after-impact behavior of FRP composite plates. Aiming to observe well-known damage/failure mechanisms which allows neglecting delamination, within the framework of the 2D CDM material model discussed earlier, some carbon plates with UD stacking sequences and the geometry of the new specimen for SAI were manufactured and tested.

Within the objective of experimentally assess the shear-after-impact behavior of FRP composite plates based on the proposed methodology presented in the previous chapter, there are some specific goals:

- Successfully execute rail tests in CFRP plates using the new specimen validating the 3-rail apparatus and demonstrating the applicability of the this novel coupon, as well as establishing a simple manufacture process for it;

- Comparison of experimental results obtained from rail in unidirectional and tensile testing in $[ \pm 45]$ angle-ply carbon laminates (undamaged);

- Execute, for each coupon, two drop-weight tests and compare their results;

- Perform rail tests in damaged (BVID) laminates and compare the results with those obtained from rail testing in pristine coupons.

Thus, achieving such specific goals enables the first step of the SAI analysis that is based on the tuning between the experimental and numerical analyses.

\subsection{Experimental Methodology}

The experimental portion of the shear-after-impact methodology is composed by a few steps culminating in a pretty straightforward approach. Actually, it is based on the successful fulfillment of each specific goal presented at the previous section. Thus, for each SAI experimental analysis one needs at least two coupons: one for the pure and the other for the damaged rail tests in the specimens. The complete SAI experimental methodology is depicted in Figure 66.

From a bigger plate two square $150 \mathrm{~mm}$ sided plates are cut. These will be the coupons used for the SAI experimental testings. Also, it is important to highlight that taking these specimens from the same plate is important to avoid variability in the results due to manufacture of the composites themselves. After being cut, the specimens are taken to a drilling machine that will make the nine circular cutouts with the support of a drilling 
Figure 66: Methodology for SAI experimental analysis.

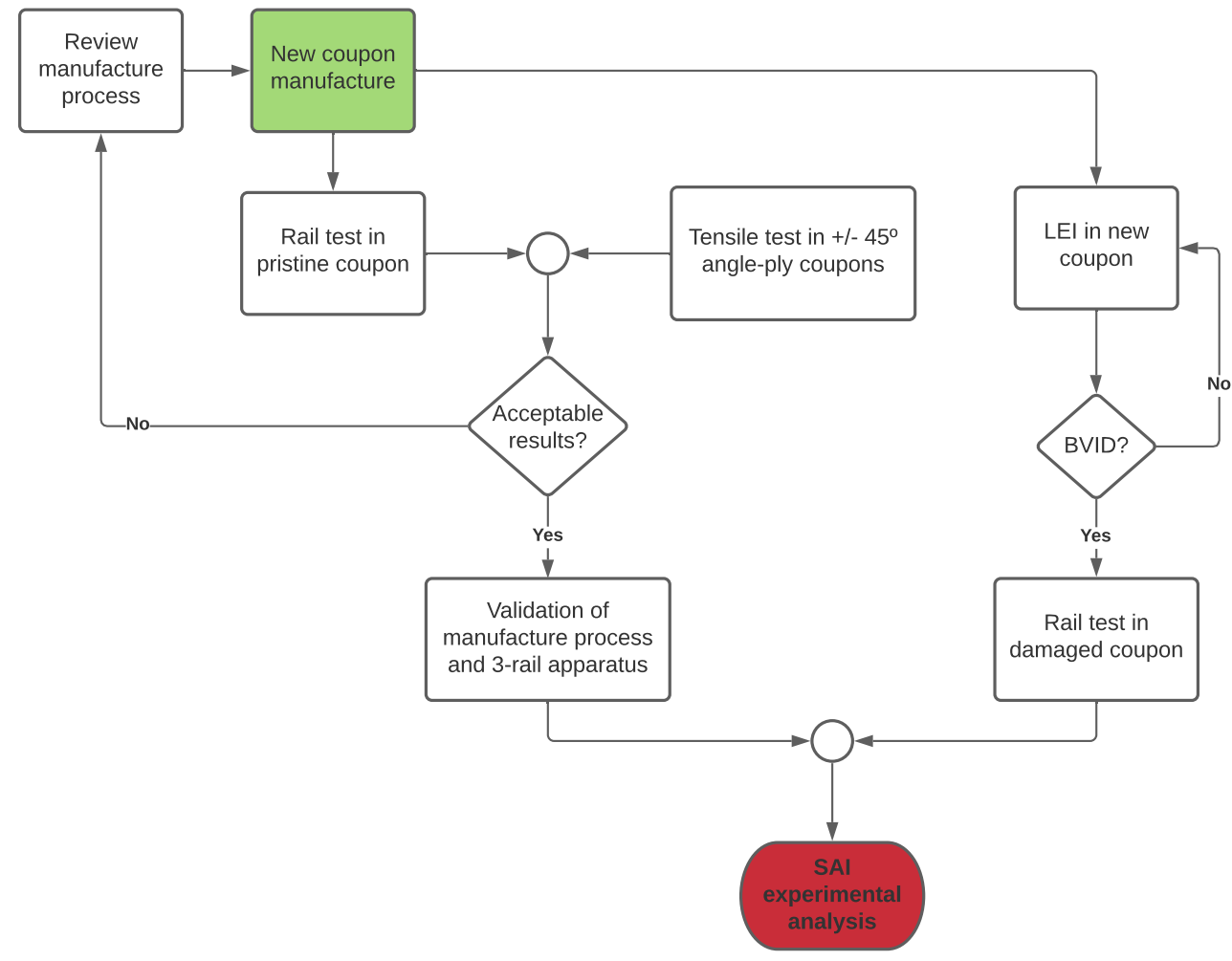

Source: by the author.

mask (two aluminum plates if holes previously drilled that makes a sandwich with the FRP plate) that serves as a template. Here handling and machining should be done with caution to avoid damage introduction due to the drilling process.

With both coupons in hands, rail testing in the one chosen not to suffer impact damage can be carried out. Results in terms of the shear stress-strain curve are obtained and compared with those obtained from tensile test in $[ \pm 45]_{n s}$ angle-ply laminates. If similar results are observed then the rail test is said to be a valid one, as well it validates the manufacture strategy adopted and the 3-rail apparatus. In the meantime, the coupon destined to be damaged by impact is taken to the drop-tower. In time, it is worth to mention that the drilling process takes place before the impact events so it can be done using the template; after damaging the structure one can found it a little warped, making it an almost impossible task to guarantee the needed distances of the holes for the coupon to fit into the 3-rail apparatus. This can cause the presence of pre-tension during assembly creating a "false" stiffness increase by the specimen. Proceeding, after performing both drop-tests, the damaged specimen is submitted to a rail test and its results are compared with those obtained for the pristine one which characterizes and finishes the SAI experimental analysis. 


\subsection{Results and Discussion}

Two CFRP plates with $[0]_{16}$ layups were chosen to be subjected under the SAI procedure. These possess 150x150x3.0 mm dimensions with nine $10.0 \mathrm{~mm}$ diameter holes weighting in average $95.8 \mathrm{~g}$. The drop-weight characteristics of the impact tests are listed in Table 14 while Figure 67 shows the results of both drop tests embodying the force, displacement and energy histories and the force-displacement curves of the events.

Table 14: Drop-test parameters for the $[0]_{16}$ laminates.

\begin{tabular}{lrrrr}
\hline Impactor mass $[\mathrm{kg}]$ & Test heigh $[\mathrm{m}]$ & Initial velocity $[\mathrm{m} / \mathrm{s}]$ & Total impact energy [J] & Impactor radius [mm] \\
\hline 4.826 & 0.117 & 1.51 & 5.53 & 8.0 \\
\hline Impactor material & & & Aluminum \\
Plate thickness $[\mathrm{mm}]$ & & 3.0 \\
\hline
\end{tabular}

Figure 67: Impact force (a), displacement (b) and energy (c) histories; force-displacement curves (d).

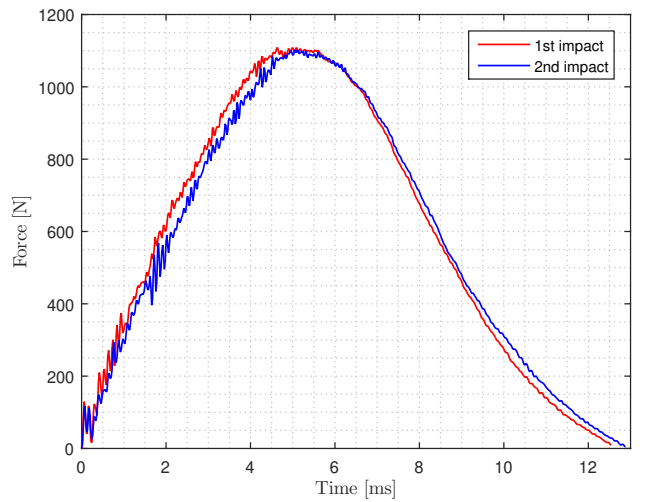

(a)

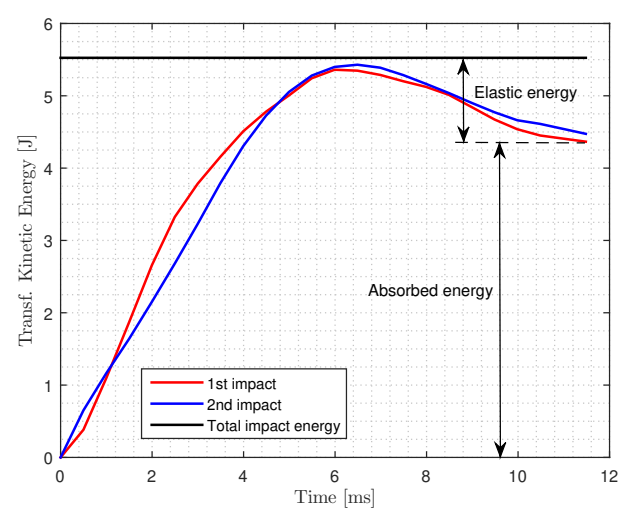

(c)

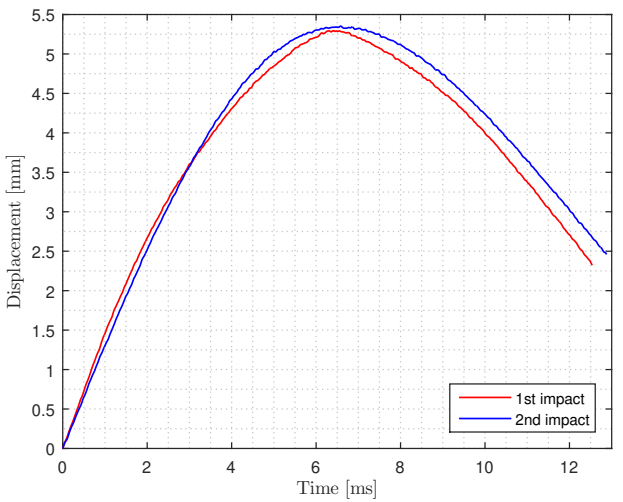

(b)

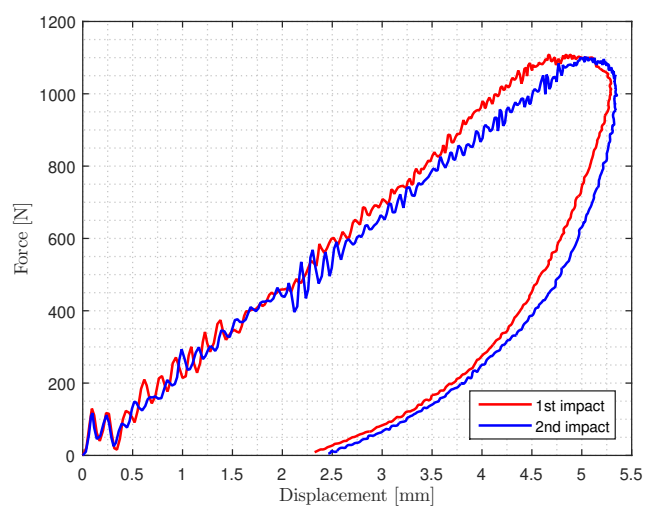

(d)

Source: by the author. 
For both tests, a sampling acquisition rate of $50 \mathrm{kSps}$ was used. Also, the impact energy level is chosen analyzing several works regarding the low-energy impact considering barely-visible impact damage, e.g. (TITA; CARVALHO; VANDEPITTE, 2008), (RIBEIRO, 2013), (FERREIRA et al., 2019) and (THORSSON; WAAS; RASSAIAN, 2018a), being cautious with the existing different nuances between the experimental apparatus used at these works and sample geometries.

It can be noticed from Fig. 67a some high-frequency oscillations at the initial portion of both curves up to approximately $1 \mathrm{~ms}$ due to the accommodation of the impactor with the target. Further oscillations observed occurs due to the introduction of damage. Also, as expected, the damage from the second impact is slightly greater than the one from the first strike. This behavior is observed in Figs. 67b and 67c too since the maximum displacement is also slightly greater in the second impact in comparison with the first one. Introduction of damage also implies in greater impact during (second event) since the plate needs to dissipate more of the transferred kinetic energy. Still, the dissipation phenomenon can be observed as the positive "to-the-left" shift in every curve (from the second impact with relation to the first one) all after damage introduction being verified for the second impact event, which occurs mainly between 1.5 and $2.2 \mathrm{~ms}$ and further oscillations are due to crack propagation through the plate. Lastly, permanent indentation is accused analyzing Fig. 67d but, since the displacement is measured with respect to the impactor and not to the plate directly, these residual displacements are only approximations of the real values. Nevertheless, it is noticed a greater value for permanent indentation in the second impact which was expected to occur. Table 15 sums up the obtained results of both impact events.

Table 15: CFRP drop-tests summary for $[0]_{16}$ laminates.

\begin{tabular}{lcc}
\hline Physical quantity & $1^{\text {st }}$ impact & $2^{\text {nd }}$ impact \\
\hline Impact duration [ms] & 12.54 & 12.88 \\
Peak force [N] & 1108.32 & 1102.06 \\
Maximum displacement [mm] & 5.29 & 5.35 \\
Indentation [mm] & 2.32 & 2.46 \\
Elastic energy [J] & 1.16 & 1.05 \\
Absorbed energy [J] & 4.37 & 4.48 \\
\hline
\end{tabular}

Afterwards, both pristine and damaged plates were subjected to a 3-rail shear tests following the ASTM D4255 standard procedure where the applied load in the central (or movable) rail by the INSTRON universal testing machine was of compression with a constant head displacement of $2.0 \mathrm{~mm} / \mathrm{min}$. Figure 68 shows the shear stress-strain obtained curves for both plates, compared with the experimental average curve of the $[ \pm 45]_{4 s}$ angle-ply coupons used previously for material shear characterization. 
Figure 68: Shear stress-strain curves: pristine/damaged rail test and \pm 45 angle-ply coupons.

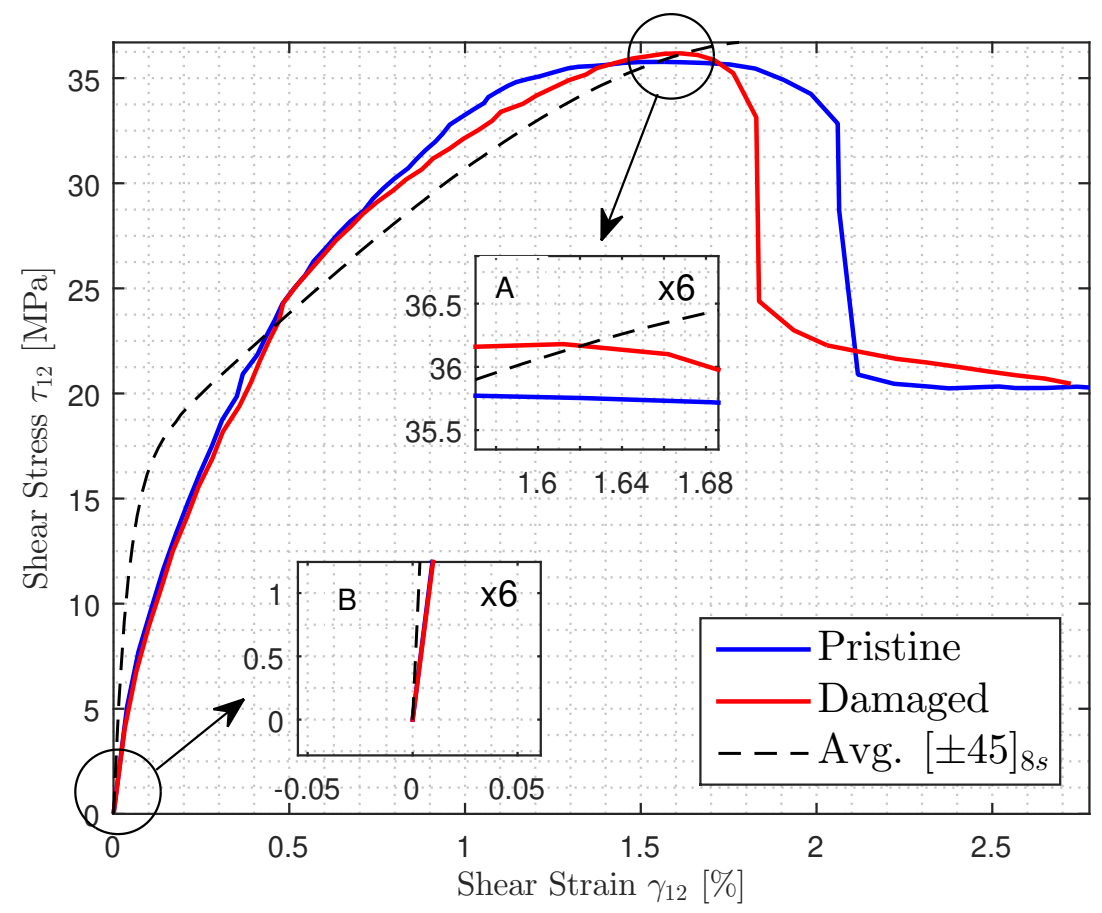

Source: by the author.

It can be noticed from Figure 68 that the damaged laminate possesses no significant reduction in stiffness then the pristine one. This is a consequence of the employed impact energy level considering both the laminates' geometrical and physical characteristics. Since the impact occurs on unidirectional thin laminates, it is observed that the bending stresses in the bottom of the plate introduce matrix cracks firstly at the non-impacted side of the coupon, which is in accordance with the expected (ABRATE, 1998). Moreover, the unique noticeable difference between both pristine and damaged coupons is that the last presents a premature failure regarding to the ultimate shear strain, which is $1.66 \%$ while for the prior is $1.83 \%$ at approximately the same shear stress level. Then, both coupons are capable of supporting further load application with the pristine one presenting a broader interval before fracture. Also, it is worth to mention that both curves for rail tests have similar characteristics with the one for the $[ \pm 45]_{4 s}$ coupons. Since only one rail test for each was performed, there is no significant statistical volume of data for definitive conclusions. Further tests are planned and performed with the aid of the numerical predictions from finite element method and results are presented latter in this text. Yet, it is notable that the rail tests resulted in a more non-linear behavior of the material compared with the bi-linear tendency of the angle-ply ones and, besides that, rail testing results provided lower stiffness values than the tensile tests in \pm 45 . The last point discussed, again, can be explained by the low quality of the pre-impregnated resin system which leads to ill-results 
regarding the matrix related properties. Also, as observed in Figure 68, maybe tensile testing in $45^{\circ}$ angle-ply laminates can provide similar levels of ultimate shear strain as UD with BVID under rail test for similar ultimate shear stress levels as well. Regarding to qualitatively results, Figure 69 shows up both pristine and impacted coupons after the rail tests.

Figure 69: Pristine (left) and impacted (right) plates after 3-rail test.

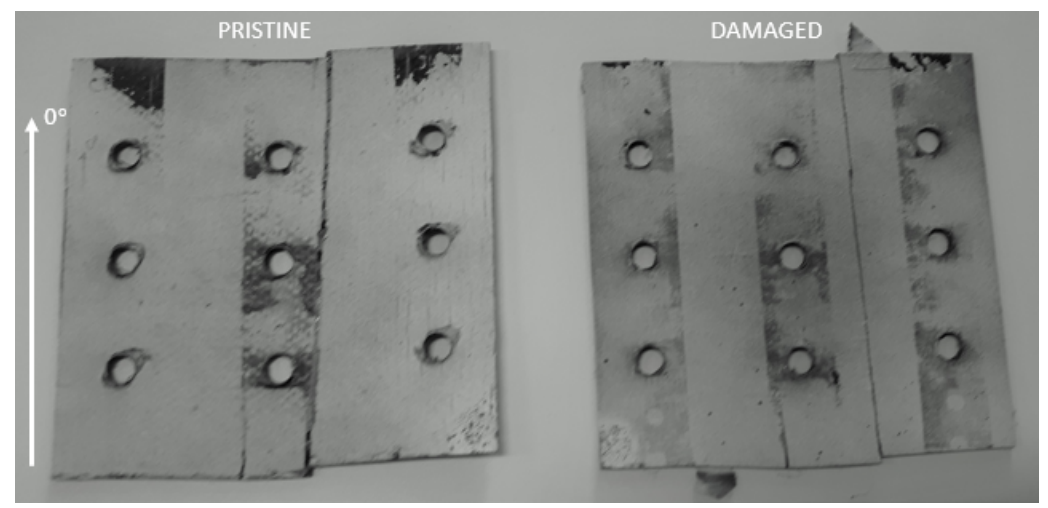

Source: by the author.

It can be observed that both coupons failed with cracks growing at the fibers' direction, parallel to load application. Also, the onset occurs nearby the moving rail for the intact specimen while for the impacted one it is observed the growth of the already existing cracks induced by impact. In time, it is worthy to mention that the performed impacts are large mass ones, as classified by Olsson (OLSSON, 1992). Moreover, no noticeable undesirable effects like screw bearing, laminate local/global buckling and stress concentration at the holes were observed during both tests and a simple shear state is noticed during DIC data treatment, as desired. Lastly, a comparison of tests performed without using the drilling mask to manufacture the cutouts is shown in Figure 70.

Figure 70: Pristine and impacted plates after 3-rail test without usage of a drilling mask.

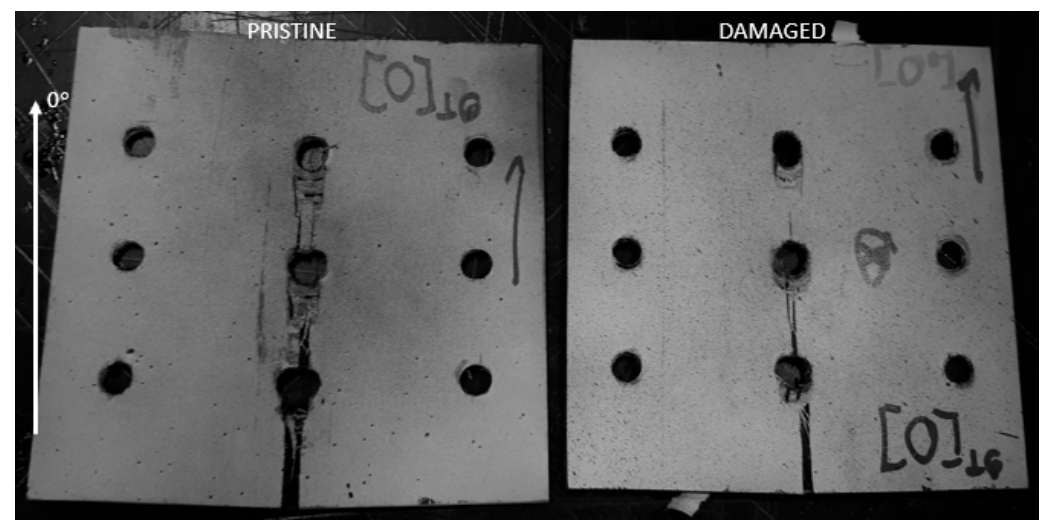

Source: by the author. 
Notice that, for both specimens, failure occurs at the holes of the central rail implying failure due to screw bearing. Furthermore, even for the damaged plate (impact point marked with a cross inside a circle) no visible crack propagation due to impact damage is observable. This occurs probably due to imprecise drilling of the holes that consequently leads to an asymmetrical grip of the 3-rail screws which ultimately translates to premature failure due to bearing and ill-results. With respect to the damage index, it is expected a low value for it since the impacted specimen possess similar stiffness and ultimate stress and strain. Also, for the studied laminate, the numerical integration of the experimental stress-strain curve provides the toughness for both pristine and damaged coupons. With this, it is possible to define the minimum allowable toughness, as discussed in Chapter 3. Thus, for this case the last is equal to $E_{D}^{\min }=0.0723 \mathrm{~J} / \mathrm{mm}^{3}$ while $E_{P}=0.5958$ $\mathrm{J} / \mathrm{mm}^{3}$ and $E_{D}=0.5078 \mathrm{~J} / \mathrm{mm}^{3}$ providing a damage index of $\mathrm{DI}_{\mathrm{SAI}}=0.1477$ indicating low impact induced damage in the sample.

Computational investigations using the finite element method software ABAQUS along with an UMAT are also made to have insight about the tendencies that should be experimentally observed. The study cases selected to assess these are of manually degrading the matrix related elastic properties $E_{22}, G_{12}$ and $\nu_{12}$ by a percentage value of the pristine ones in the damaged region experimentally estimated. The degradation percentages are of $10 \%, 30 \%$ and $50 \%$ in relation with the original ones. The FE model mimics the characteristics of the $\left[0^{\circ}\right]_{16}$ 3-rail test specimen where a simple rectangular geometry, equal to the one between the central and one free rail, is chosen for the analyses possessing 27.5 $\mathrm{mm}$ width, $150 \mathrm{~mm}$ length and $2.0 \mathrm{~mm}$ thickness meshed with S8R quadrilateral shell elements with 8 nodes, $6 \mathrm{DoF} /$ node and reduced integration. The boundary conditions are of encastre at the fixed rail side and pre-defined displacement in the $x$-direction of 2.0 $\mathrm{mm}$ at the free rail side. The region of impact is modeled simply as a partition of the part model in ABAQUS with the corresponding degraded properties as depicted in Figure 71.

Figure 71: FE model of the $\left[0^{\circ}\right]_{16}$ 3-rail test specimen.

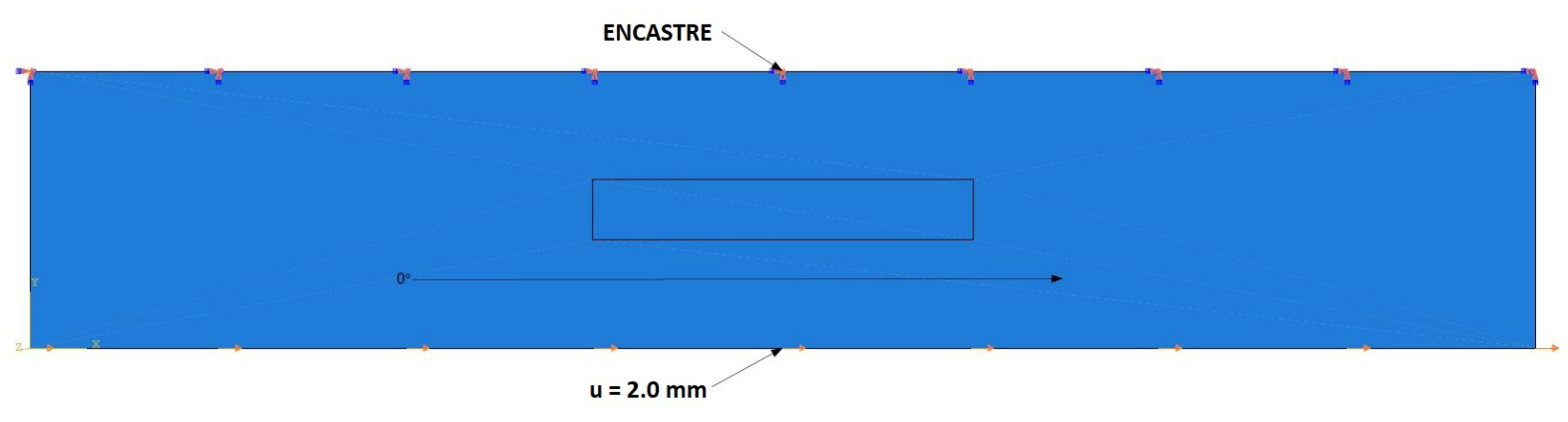

Source: by the author.

Notice that the central region is where the degradation is located and if no damage is introduced, one simply deletes this extra region for the pristine FE model of the 3-rail 
test. About the estimation of the damaged region in the coupon the methodology adopted is a semi-analytical approach following the performed drop-test and Abrate (ABRATE, 1998). The first is made by a visual inspection aided by a magnifier trying to estimate the damage extension. Also, according to Abrate the damaged region after a LEI follows an elliptical shape that obeys,

$$
\begin{aligned}
& a=2 \sqrt{\pi}\left(\frac{D_{11}}{m}\right)^{1 / 4}[2(A+1)]^{1 / 8} \sqrt{t}, \\
& b=\frac{a}{\left(D_{11} / D_{22}\right)^{1 / 4}},
\end{aligned}
$$

in which $a$ and $b$ are the the ellipse radii as usual. Moreover, $D_{i j}$ are the elements of the bending-torsion stiffness matrix from CLT, $m$ is the specimen mass and $t$ is the impact duration time that can be estimated or experimentally obtained. In this approach, the last is adopted. Also, the parameter $A$ is interpreted as an stiffness ratio that is given as,

$$
A=\frac{D_{12}+2 D_{66}}{\sqrt{D_{11} D_{22}}} .
$$

Applying this methodology, the values obtained for the ellipse radii are of $a=20$ $\mathrm{mm}$ and $b=8 \mathrm{~mm}$ for the experimentally tested coupon. These are estimated values but are probably near the real value since the visual inspection provided something next to these, with values between $\approx 16 \mathrm{~mm}$ and $\approx 6 \mathrm{~mm}$ for $a$ and $b$ respectively. Of course, there is internal damage that can not be noticed with such rudimentary equipment but is currently the best that can be done at GEA's facilities. So, being conservative, the values adopted for the FE model were those obtained using Abrate's approach. There are also two important things to mention. Notice that this region is modelled as a rectangle and not as an ellipse; this is due to the easiness of meshing the model and one should not obtain very different tendencies if adopts the elliptical region instead of the rectangular one. Another thing is that the different degradation values used here for the study does not represent necessarily the size of the damaged region. Of course, as you increase the transferred kinetic energy the size of this region also increases. For this study this is not considered since it would add another parameter to consider during the analyses. The main goal of the subsequent computational simulations is to investigate the tendencies that should be experimentally observed in shear-after-impact testing. To account for the different sizes of the damaged region more drop-tests are recommended.

Starting the investigations, firstly, a comparison depicted in Figure 72 between the damage onset envelopes used in the current work and Ferreira's one is made. It is noticeable that both envelopes capture similar behaviors, including the ultimate shear stress and strain prediction. Even though, for the current envelope the stress plateau is not present (for the FE $[ \pm 45]_{4 s}$ model) as it is in Ferreira's envelope in which the stress-strain curve stays linear-elastic until it reaches it. This feature is not present for 
the angle-ply computational model using the current work envelope. Also, for the rail test model, ultimate and fracture shear stresses are different from each other even though the ultimate shear strain remains almost the same. Yet, other discrepancy between the two models is in the value of the fracture shear strain, which is also greater for the rail one.

Figure 72: Comparison between shear stress-strain curves using the current work damage onset envelope and Ferreira (2014) for tensile \pm 45 angle-ply and rail test laminate.

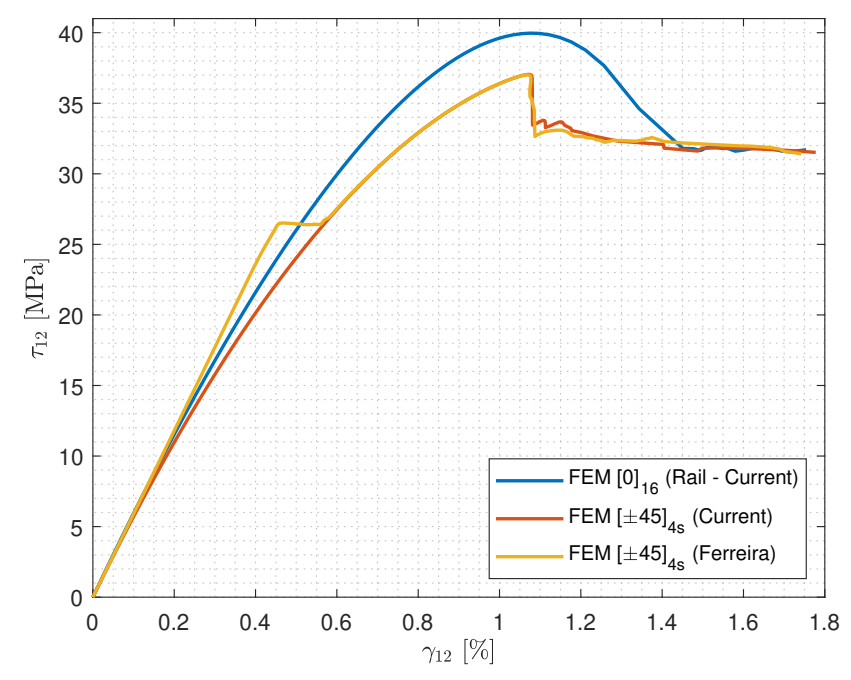

Source: by the author.

Moreover, an interesting comparison is the one among the damage variables $d_{2}$ and $d_{6}$ evolution during the FE analysis, as shown in Figure 73.

Figure 73: Damage variables evolution during FEA of rail tests (a) and tensile \pm 45 angle-ply (b) models.

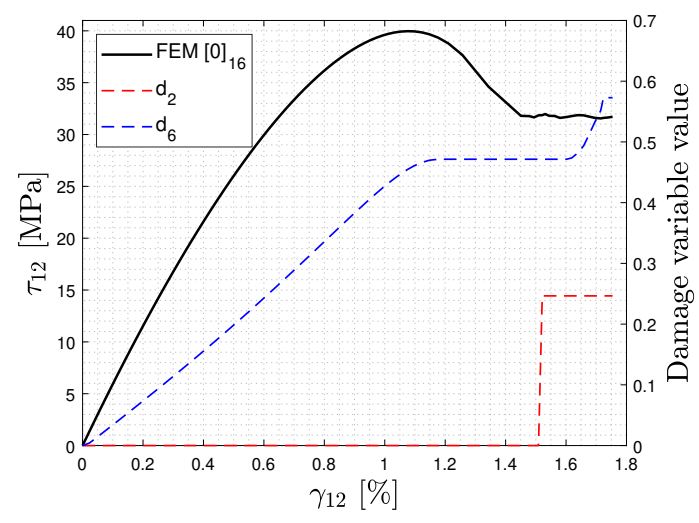

(a)

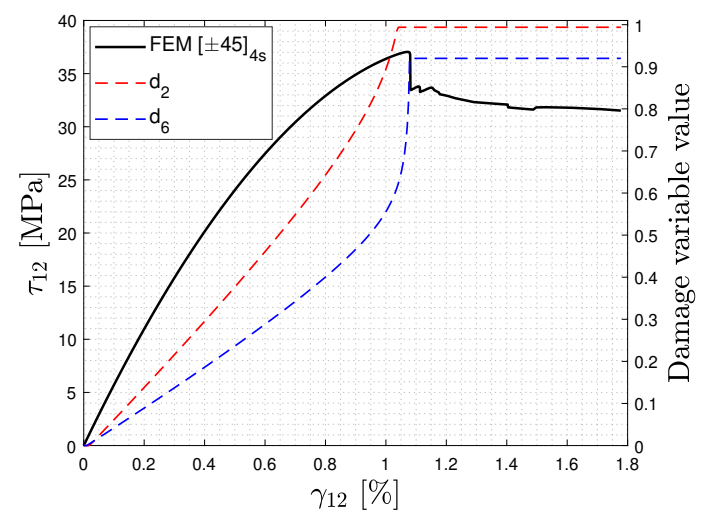

(b)

Source: by the author. 
In it, the damage variable behavior under simple (rail test) and pure (tensile angle-ply) shear states are compared. Notice that for the case of pure shear the damage variable $d_{2}$ is not activated until the fracture shear stress is reached. On the other hand, the damage variable $d_{6}$ is activated at the beginning, growing linearly until the ultimate shear stress where it remains constant and grow up again a little bit after the activation of $d_{2}$. For the pure shear state, both damage variables are activated at the beginning and grows linearly until the ultimate/fracture shear stresses are reached (both are the same in this case), where both of them grows to their greater values. For $d_{6}$, this growth is abrupt while for $d_{2}$ is smoother. Qualitatively, Figure 74 shows up the distribution of $d_{6}$ for the cases of simple and pure shear states in ABAQUS at the ultimate shear stresses of both models.

Figure 74: Damage variable $d_{6}$ distribution at ultimate shear stress for rail test (a) and tensile \pm 45 angle-ply (b) computational models.
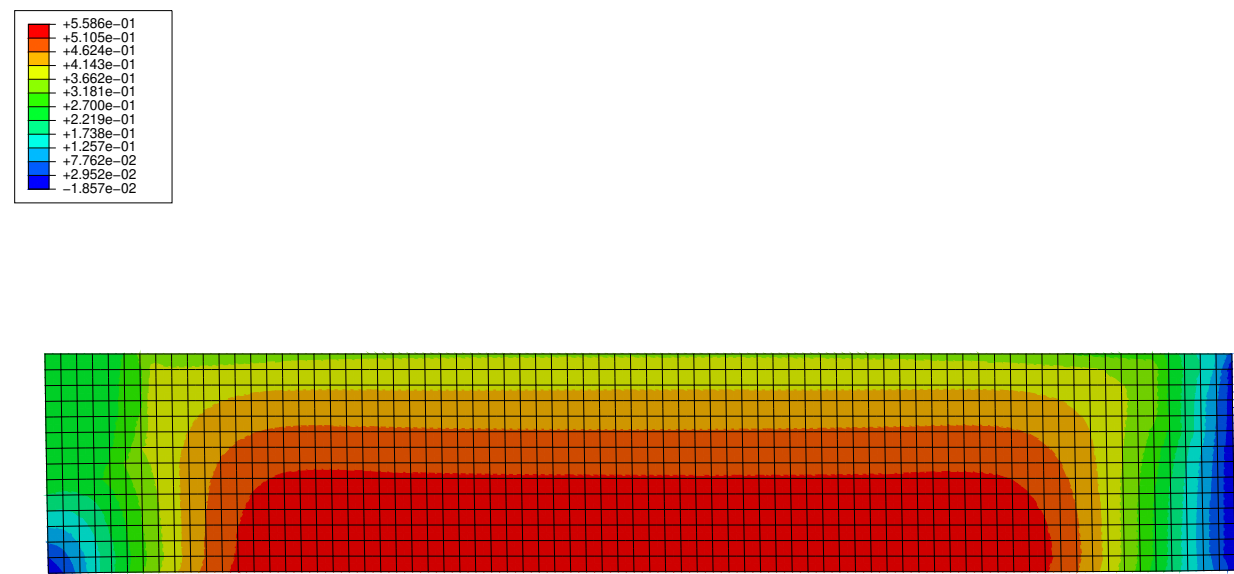

(a)
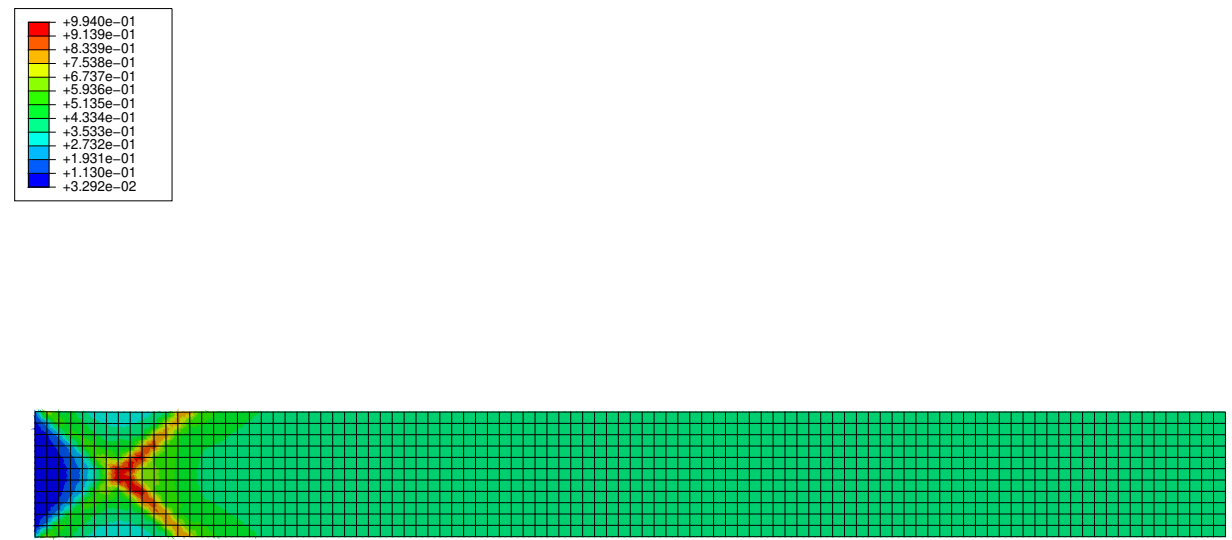

(b)

Source: by the author. 
Table 16 sums up the commented results for the ultimate and fracture shear stresses and strains, compiled with the experimental results obtained for rail and tensile angle-ply tests.

Table 16: Results summary for simple (rail) and pure (angle-ply) shear comparisons: current work and Ferreira's (2014) envelopes

\begin{tabular}{|c|c|c|c|c|c|}
\hline Model & Envelope & $\begin{array}{c}\text { Ultimate shear } \\
\text { stress }[\mathrm{MPa}]\end{array}$ & $\begin{array}{c}\text { Ultimate shear } \\
\text { strain }[\%]\end{array}$ & $\begin{array}{c}\text { Fracture shear } \\
\text { stress }[\mathrm{MPa}]\end{array}$ & $\begin{array}{c}\text { Fracture shear } \\
\text { strain }[\%]\end{array}$ \\
\hline Rail $\left[0^{\circ}\right]_{16}$ & Current work & 39.97 & 1.076 & 31.8 & 1.451 \\
\hline $\begin{array}{l}\text { Angle-ply } \\
\text { layup }\left[ \pm 45^{\circ}\right]_{4 s}\end{array}$ & Current work & 37.04 & 1.075 & 37.04 & 1.075 \\
\hline $\begin{array}{l}\text { Angle-ply } \\
\text { layup }\left[ \pm 45^{\circ}\right]_{4 s}\end{array}$ & Ferreira (2014) & 36.99 & 1.074 & 36.99 & 1.074 \\
\hline $\begin{array}{l}\text { Exp. value } \\
\text { rail test }\end{array}$ & $\mathrm{n} / \mathrm{a}$ & 35.77 & 1.498 & 32.84 & 2.06 \\
\hline $\begin{array}{l}\text { Exp. value } \\
\text { angle-ply }\end{array}$ & $\mathrm{n} / \mathrm{a}$ & 37.71 & 1.86 & 37.71 & 1.86 \\
\hline
\end{tabular}

Regarding the shear stress-strain curves for the damaged models, Figure 75 shows up the obtained results.

Figure 75: Computational shear stress-strain curves: pristine/damaged rail tests FE model results.

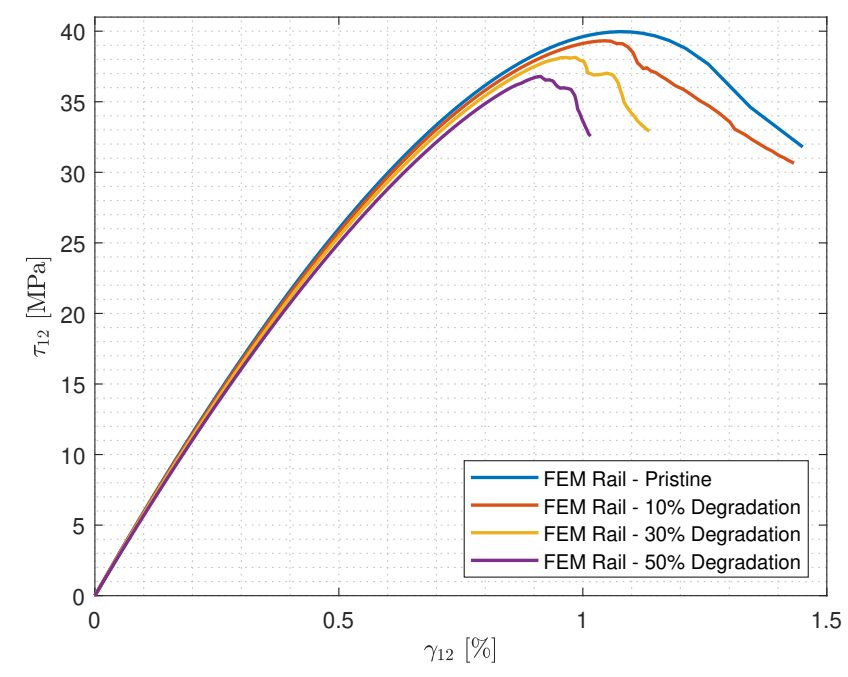

Source: by the author.

As can be noticed, for higher degradation percentages (i.e. greater impact induced damage) both the ultimate and fracture shear strain and stresses are lower. Of course, this represents a premature failure of the specimen as you increase damage. Also, a decrease in the stiffness is observed as should be expected. Thus, for greater degradation, it is observed lower values of the SAI damage index; these results are compiled quantitatively in Table 17. 
Table 17: Shear-after-impact damage index FEM results

\begin{tabular}{cccc}
\hline Damage Index & $10 \%$ Degradation & 30\% Degradation & $50 \%$ Degradation \\
\hline $\mathrm{DI}_{\mathrm{SAI}}$ & 0.0416 & 0.3062 & 0.4259 \\
\hline
\end{tabular}

As verified, the values of the damage index grows up for higher degradation. Yet, it can be seen that, considering the methodology applied and the simplifications adopted for the computational model, the experimental drop-weight test induced damage that degraded the material elastic properties in somewhere between $10 \%$ and $30 \%$ of the undamaged values. Furthermore, it is worth to mention that for all $\mathrm{FE}$ analyses mesh convergence is considered for a maximum relative error of $5 \%$ between the variables of interest from one result to the other.

In this chapter, the impact and post-impact behavior of carbon-fiber reinforced composite plates with $[0]_{16}$ layup under the barely-visible impact damage limit or, simply, low-energy impact, was experimentally assessed considering for the post-impact event a simple shear stress state. For this, a new specimen that fits both drop-weight and 3-rail tests is proposed and the experiments follows a straightforward procedure of analysis. Some key aspects are noticed during the impact events that introduce damage in the coupon, that are:

- Time duration of the $2^{\text {nd }}$ impact increases as a consequence of the necessity of dissipate more of the transferred kinetic energy culminating in a positive shift in all force/displacement curves;

- Damage introduction due to the $2^{\text {nd }}$ impact event is slightly more severe;

- Permanent indentation grows for the $2^{\text {nd }}$ impact even though the experimental value is only an approximation of the real one.

Regarding to the shear-after-impact analysis, more experiments need to be made. Since only one set of two plates are used for comparison using the proposed methodology for SAI, some tendencies should be observed but this can only be used as a basis for further testing. These are performed after FE analysis, following a procedure of gradually degrading the material elastic properties related to the matrix, to aid the strategy for SAI. Nevertheless, both the pristine and impacted experimentally tested coupons show remarkable similarity in the stress-strain curves, with the second presenting approximately the same ultimate shear stress and strain values as compared with the $45^{\circ}$ angle-ply specimens which can imply that the last ones can underestimate these values. Also a highly non-linear behavior is noticed for the rail test results compared with the bi-linear trending of the angle-ply laminates. As expected, failure occurs at the already existing crack due to 
its growth in the fiber direction for the damaged plate, in which two low-energy impacts in the BVID range are performed as for the pristine one, crack onset and propagation occur at the neighborhood of the central rail. Furthermore, the angle-ply laminate under tension accuses greater shear modulus than the ones submitted to rail testing. Lastly, the DIC technique was fully capable of capturing the desired simple shear state as depicted in Figures 76 and 77.

Figure 76: DIC strain field of pristine coupon at ultimate (a) and fracture (b) shear stresses; FEM strain field of pristine coupon at fracture shear stress (c).

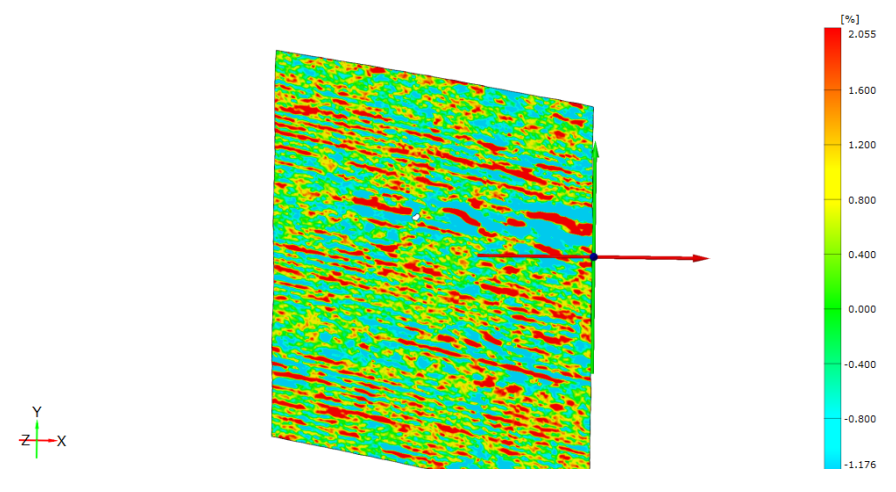

(a)

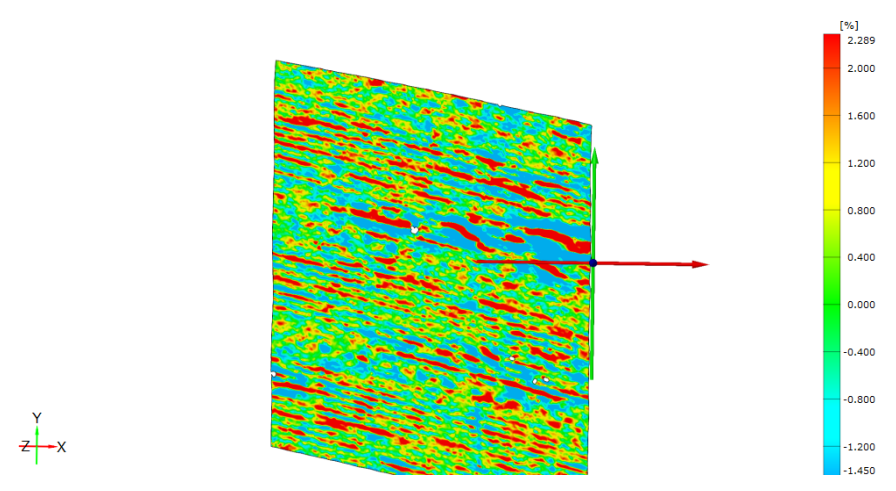

(b)

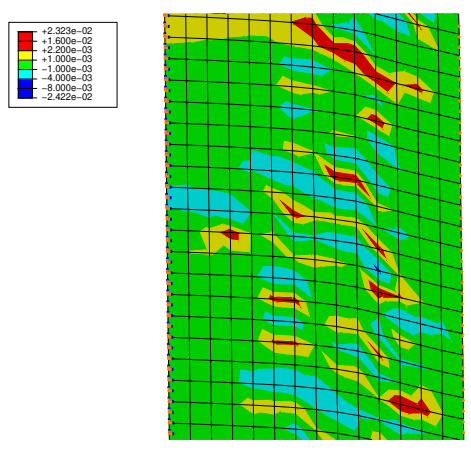

(c)

Source: by the author.

Notice that the finite element analysis shows the instant of fracture initiation that 
Figure 77: DIC strain field of damaged coupon at ultimate (a) and fracture (b) shear stresses; FEM strain field of damaged coupon with $50 \%$ degradation at fracture shear stress (c).

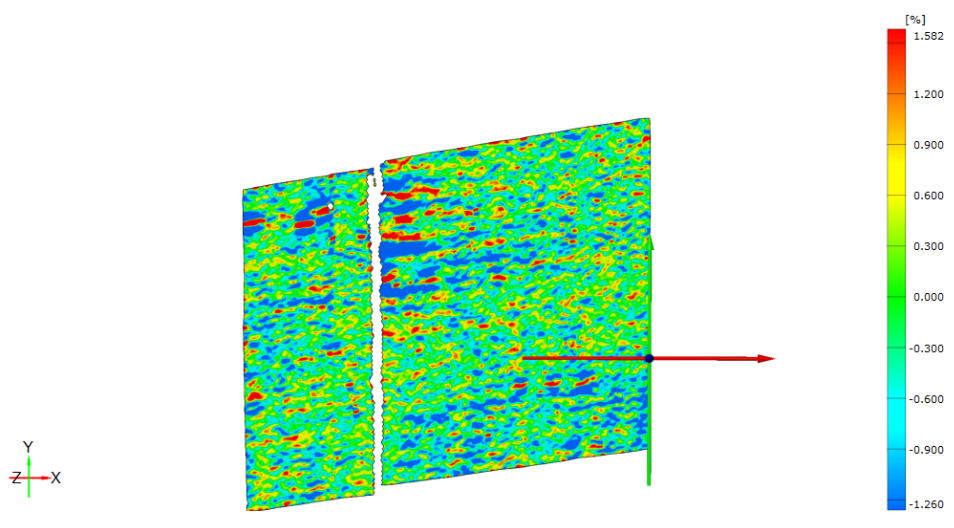

(a)

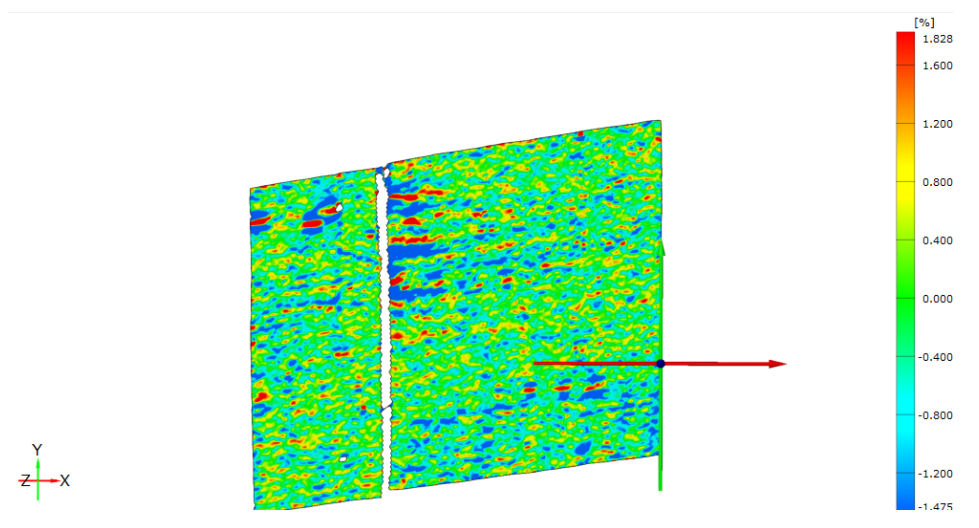

(b)

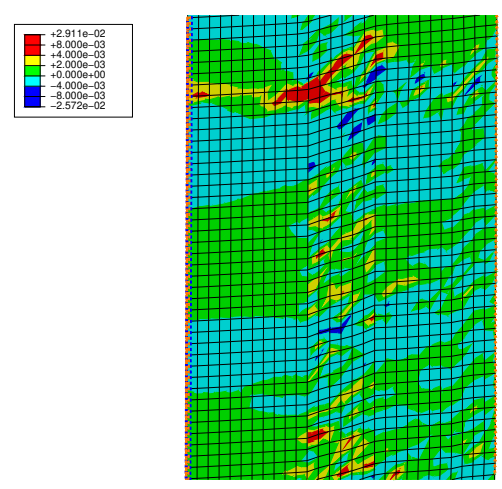

(c)

Source: by the author.

are shown in both figures, since the model does not account for element loss during the simulation. Also, it is concluded that the rail apparatus and the analysis procedure are promising in being used as tools for residual strength assessment. Moreover, since the low-energy impact is simulated only by the degradation of the matrix elastic properties, 
Figure 77c can only evaluate qualitatively the expected strain field and, so, the obtained values are not necessarily representative of the real SAI experimental test. 



\section{FINAL REMARKS}

\subsection{Conclusions}

The main goal of this work was of conducting an investigation of the SAI behavior of fiber-reinforced polymer composite materials since there are no documented works aiming this objective in the literature. In this context, some analyses were realized and conclusions can be made trying to pave the path to fulfill the existing gap in the area.

Firstly, a literature review on the relevant subjects was performed. In it, attention is given to damage and failure of composite materials. Also, the behavior of these materials under impact loadings is reviewed. The range of LVI, within the optics of BVID, is defined by the mode-one energy ratio criterion where the terminology is modified for LEI, a better one. Within the framework of the finite element method, a meso-scale two-dimensional material model accounting for progressive failure is chosen to assess the behavior of FRP composites. This criterion is based on continuum damage mechanics, being thermodynamic compatible and, consequently, obeys its laws. It is worth to mention that the present author thinks that meso-scale material models are unable to capture separately fiber, matrix and fiber-matrix interface failures. This is due to the ply homogenization done in the first step of meso-scale analyses, the common feature of all these models. Still, characterization experiments are performed to identify material properties and, regarding to the failure criterion parameters accounting damage evolution and coupling, cyclic tests were done. Also, the reliability of the hand-layup manufactured laminates is taken care by monitoring the cure cycle, assessing the fiber and matrix volume fractions and by adopting systematic procedures during its fabrication and machining.

Furthermore, a shear-after-impact experimental analysis approach was proposed based mainly on Medeiro's (MEDEIROS, 2016) work on flexure-after-impact. In it, pristine specimens are compared with damaged ones to assess its post-impact shear behavior. A new coupon is idealized for these tests that are performed with a drop-weight machine and, after that, with the 3-rail test apparatus. Also, the coupon geometry makes possible to perform impacts in two different points, with different energy levels if desired, and it suits the Boeing fixture for drop-weight testing.

Additionally, some preliminary study cases were carried out. Two quasi-static tensile tests and one impact drop-weight test were chosen for the initial investigations. The material and tests were firstly studied and performed by Tita (TITA, 2003), followed by Ferreira (FERREIRA, 2014). Also, for the tensile tests with fibers oriented in the loading direction, comparison with the already obtained results of this work is done as well. Good qualitative and quantitative responses are documented. In the case of the shear test, 
interesting results were obtained and good prediction capabilities of the FE model are registered. Among with it, the FE model was tested with the pure linear-elastic behavior, i.e. without damage, as well and similar results were obtained. Moreover, it is important to notice that within a quasi-static response as proposed by Cristoforou and Yigit (YIGIT; CHRISTOFOROU, 2007) one can use the UMAT to describe impact tests, i. e. an implicit numerical solution, neglecting inertia terms. This approach turns easier the link between using the outputs from impact simulations as inputs for the shear (after impact) ones and can be the adopted numerical strategy in future works.

For the shear-after-impact methodology application drop-tests were realized in $\left[0^{\circ}\right]_{16}$ specimens to introduce damage. After, the damaged an undamaged coupons passed trough rail testing to obtain the shear stress-strain curves for both of them. These are used to propose a energy-based damage index to serve as a metric of the residual strength for SAI of FRP laminates. Moreover, finite element analysis were realized for gradually growing material elastic properties degradation to observe the tendencies that should be expected in further experiments and to evaluate the potentialities and limitations of the employed material model with the current work damage onset envelope and the one proposed by Ferreira (FERREIRA, 2014).

Also, an experimental drop-weight test was performed in an Al 7075-T6 metallic plate with the apparatus present at the GEA facility. Some data treatment was done with the Savitzky-Golay filtering combined with a genetic optimization implemented algorithm to calibrate the drop-tower. This opens the door for future works using the experimental setup at EESC/USP.

Finally, the main goal of this Master's dissertation was achieved, with novel guidelines for the analysis of shear-after-impact (low-energy and face-on) behavior of FRP composites being obtained towards the application of the proposed methodology in which the last had its potentialities and limitations explored. In general, the SAI test is complementary to compression- and flexure-after-impact tests. In this sense, the ideal is to evaluate the post-impact structural integrity by combining the results of all three analyses and, then, having a more complete damage metric to assess the residual strength or other property of interest.

\subsection{Future Works}

There are several research lines and improvements that can be explored based on the study and results of this work. In reference of the material model application, firstly, it would be interesting to use a compression test device (e.g. IITRI Compression Test) to perform these experiments in $[0]_{n}$ and $[90]_{n}$ coupons to obtain these stress-strain curves and from those identify the related parameters and evolution laws by means of the secant method. Certainly this will improve its predictions capabilities. Also, considering 
delamination onset and propagation its a suggestion for future works. Considering a 3D material model using, for example, the Cohesive Zone Method (CZM) or the Virtual Crack Closure Technique (VCCT) is an important improvement. It is important to remember that both these methods were developed to analyze metallic materials and does not possess a physical meaning when applied to composite laminated structures, besides its wide application and good prediction capabilities. One could use other strategies such as phenomenological ones or even starting from theoretical approaches considering first principles for equations derivations. Still, an interesting study is the one involving carrying out more off-axis cyclic tests to obtain better results regarding to the fluctuation of the coupling parameter b through these cycles and also its dependency with the orientation angle $\theta$. This should improve results from FE simulations of low-energy impacts and others.

Regarding to the definition of what a low-energy (or velocity) impact is there are some suggestions as well. The author thinks that a good strategy would that of planning a series of several drop-weight tests to limit the range of some dimensionless parameters that should define the LEI considering a precise definition of barely-visible impact damage. For example, one expects to see different damage/failure modes for a LEI in unidirectional than in cross-ply laminates with the prior being dominated by matrix cracks while the last presenting both matrix cracks and delamination. This approach would use the dimensionless parameters derived by Cristoforou and Yigit (YIGIT; CHRISTOFOROU, 2007) but with a different focus than the presented in their works. For them, the objective is to characterize impacts regarding to the type of dynamic response that is expected to arise from the event. Thus, the concern is in identifying the equations of motion and its solutions in terms of force and displacement histories, for example, while here the focus would be of characterizing LEI considering the ranges in which these parameters varies in the definition of BVID.

Yet, it would be of good practice to improve the experimental evaluation with some more sophisticated damage detection techniques such as ultrasound, C-scan and scanning electron microscopy, to name a few. Regarding the methodology, with the 3D material model to account for delamination it is left for future works the shear-after-impact analysis for cross-ply, angle-ply and quasi-isotropic laminates. The evaluation of these structures with a damage metric is of unique importance for Structural Health Monitoring issues. Lastly, an interesting study is the one involving the usage of a 2-rail testing device. Comparing results of the 3 -rail and the 2-rail tests which induces a simple and a pure shear stress state respectively would be an interesting study and could provide good results for residual strength assessment. 



\section{BIBLIOGRAPHY}

ABRATE, S. Impact on laminated composite materials. 1991.

Impact on composite structures. [S.l.]: Cambridge university press, 1998.

ADAMS, D. O. et al. A v-notched rail shear test for composite laminates. In: Proceedings of the Annual Conference and Exposition on Experimental and Applied Mechanics. [S.l.: s.n.], 2002.

ANDERSON, T. L. Fracture mechanics: fundamentals and applications. [S.l.]: CRC press, 2005.

ANGELO, M. V. Análise quase-estática de estruturas escalonadas laminadas em material compósito via modelo fenomenológico de falhas e elementos finitos estendidos: desenvolvimento de uma ferramenta computacional. 2018. Tese (Doutorado) — Universidade de São Paulo, 2018.

BAERE, I. D.; PAEPEGEM, W. V.; DEGRIECK, J. Design of a modified three-rail shear test for shear fatigue of composites. Polymer Testing, Elsevier, v. 27, n. 3, p. 346-359, 2008.

BOGENFELD, R.; KREIKEMEIER, J.; WILLE, T. Review and benchmark study on the analysis of low-velocity impact on composite laminates. Engineering Failure Analysis, Elsevier, v. 86, p. 72-99, 2018.

BOUVET, C.; RIVALLANT, S. Damage tolerance of composite structures under low-velocity impact. In: Dynamic Deformation, Damage and Fracture in Composite Materials and Structures. [S.1.]: Elsevier, 2016. p. 7-33.

BOUVET, C.; RIVALLANT, S.; BARRAU, J.-J. Low velocity impact modeling in composite laminates capturing permanent indentation. Composites Science and Technology, Elsevier, v. 72, n. 16, p. 1977-1988, 2012.

CALIRI JUNIOR, M. F. Contribution to the theory of plates: structural analyses of laminated composites and sandwich structures via unified formulations. 2017. Tese (Doutorado) — Universidade de São Paulo, 2017.

CALIRI JUNIOR, M. F.; FERREIRA, A. J.; TITA, V. Through-the-thickness stress profiles in laminated composite and sandwich structure plates via unified formulation. Composites Part B: Engineering, Elsevier, v. 107, p. 29-42, 2016.

A new finite element for thick laminates and sandwich structures using a generalized and unified plate theory. International Journal for Numerical Methods in Engineering, Wiley Online Library, v. 109, n. 2, p. 290-304, 2017.

CALIRI JUNIOR, M. F.; TITA, V.; FERREIRA, A. J. M. New generalized unified solution method for thin laminated plates. AIAA Journal, American Institute of Aeronautics and Astronautics, v. 54, n. 8, p. 2556-2560, 2016.

CANTWELL, W. J.; MORTON, J. The impact resistance of composite materials - a review. composites, Elsevier, v. 22, n. 5, p. 347-362, 1991. 
CHABOCHE, J. Description thermodynamique et phénoménologique de la viscoplasticité cyclique avec endommagement (publication no. 1978-3). 92320 chatillon. France: Office National dEtudes et de Recherches Aérospatiales, 1978.

CHABOCHE, J.; LEMAITRE, J. Aspects phénoménologiques de la rupture par endommagement. Journal de Mécanique Appliquée, v. 2, n. 3, p. 317-365, 1978.

CHABOCHE, J.-L. Continuous damage mechanics - a tool to describe phenomena before crack initiation. Nuclear Engineering and Design, Elsevier, v. 64, n. 2, p. 233-247, 1981.

CHRISTOFOROU, A. P. Impact dynamics and damage in composite structures. Composite structures, Elsevier, v. 52, n. 2, p. 181-188, 2001.

CHRISTOFOROU, A. P.; ELSHARKAWY, A. A.; GUEDOUAR, L. H. An inverse solution for low-velocity impact in composite plates. Computers \& Structures, Elsevier, v. 79, n. 29-30, p. 2607-2619, 2001.

CHRISTOFOROU, A. P.; YIGIT, A. S. Characterization of impact in composite plates. Composite structures, Elsevier, v. 43, n. 1, p. 15-24, 1998.

Scaling of low-velocity impact response in composite structures. Composite Structures, Elsevier, v. 91, n. 3, p. 358-365, 2009.

COLHERINHAS, G. B. Ferramenta de otimização via algoritmos genéticos com aplicações em engenharia. 2016 — Universidade de Brasília, 2016.

CORDEBOIS, J.; SIDOROFF, F. Endommagement anisotrope en élasticité et plasticité. JMTA, Numéro spécial, p. 45-60, 1982.

DANIEL, I. M.; ISHAI, O. Engineering mechanics of composite materials. [S.l.]: Oxford university press New York, 1994. v. 3.

DAVILA, C. G.; CAMANHO, P. P.; ROSE, C. A. Failure criteria for frp laminates. Journal of Composite materials, Sage Publications Sage CA: Thousand Oaks, CA, v. 39, n. 4, p. 323-345, 2005.

DONADON, M. et al. A progressive failure model for composite laminates subjected to low velocity impact damage. Computers \& Structures, Elsevier, v. 86, n. 11-12, p. $1232-1252,2008$.

FENG, Y. et al. Experimental investigation on different positional impact damages and shear-after-impact (sai) behaviors of stiffened composite panels. Composite Structures, Elsevier, v. 178, p. 232-245, 2017.

Investigation on impact damage evolution under fatigue load and shear-after-impact-fatigue (saif) behaviors of stiffened composite panels. International Journal of Fatigue, Elsevier, v. 100, p. 308-321, 2017.

FERREIRA, G. F. et al. Development of a finite element via unified formulation: Implementation as a user element subroutine to predict stress profiles in composite plates. Thin-Walled Structures, Elsevier, v. 157, p. 107107, 2020. 
FERREIRA, G. F. O. Estruturas de material compósito sob carregamento de tração e impacto: avaliação de um modelo de material. 2014 - Universidade de São Paulo, 2014.

FERREIRA, G. F. O. et al. Computational analyses of composite plates under low-velocity impact loading. Materials Today: Proceedings, Elsevier, v. 8, p. 778-788, 2019.

GIBSON, R. F. Principles of composite material mechanics. 3. ed. [S.l.]: Taylor \& Francis, CRC Press, 2012. (Mechanical Engineering: A Series of Textbooks and Reference Books).

GONZÁLEZ, E. et al. Simulation of drop-weight impact and compression after impact tests on composite laminates. Composite Structures, Elsevier, v. 94, n. 11, p. 3364-3378, 2012.

HAHN, H.; TSAI, S. On the behavior of composite laminates after initial failures. Journal of Composite Materials, Sage Publications Sage CA: Thousand Oaks, CA, v. 8, n. 3, p. 288-305, 1974.

HASHIN, Z.; ROTEM, A. A fatigue failure criterion for fiber reinforced materials. Journal of composite materials, Sage Publications Sage CA: Thousand Oaks, CA, v. 7, n. 4 , p. 448-464, 1973.

HERAKOVICH, C. T. Mechanics of fibrous composites. [S.l.]: John Wiley \& Sons, 1998.

HINTON, M.; KADDOUR, A. S.; SODEN, P. D. Failure criteria in fibre reinforced polymer composites: the world-wide failure exercise. [S.l.]: Elsevier, 2004.

HODGKINSON, J. M. Mechanical testing of advanced fibre composites. [S.l.]: Elsevier, 2000.

HUBBARD, J. H.; HUBBARD, B. B. Vector calculus, linear algebra, and differential forms: a unified approach. [S.l.: s.n.], 2015.

JACOB, F.; TED, B. A first course in finite elements. [S.l.]: Wiley, 2007.

JOHNSON, A. F.; PICKETT, A. K.; ROZYCKI, P. Computational methods for predicting impact damage in composite structures. Composites Science and Technology, Elsevier, v. 61, n. 15, p. 2183-2192, 2001.

JONES, R. M. Mechanics of Composite Materials. 2. ed. [S.l.]: Taylor \& Francis, 1999. (Materials Science \& Engineering Series).

KACHANOV, L. On the creep fracture time, izv. Akad Nauk USSR Otd. Tekh., v8, p. 26-31, 1958.

Introduction to continuum damage mechanics. [S.l.]: Springer Science \& Business Media, 2013. v. 10.

KADDOUR, A.-S.; HINTON, M. Maturity of 3d failure criteria for fibre-reinforced composites: Comparison between theories and experiments: Part b of wwfe-ii. Journal of Composite Materials, Sage Publications Sage UK: London, England, v. 47, n. 6-7, p. 925-966, 2013. 
KRAJCINOVIC, D.; FONSEKA, G. The continuous damage theory of brittle materials. J. appl. Mech, v. 48, n. 4, p. 809-824, 1981.

KRAJCINOVIC, D.; SILVA, M. A. G. Statistical aspects of the continuous damage theory. International Journal of Solids and Structures, Elsevier, v. 18, n. 7, p. 551-562, 1982.

LADEVEZE, P.; LEDANTEC, E. Damage modelling of the elementary ply for laminated composites. Composites science and technology, Elsevier, v. 43, n. 3, p. 257-267, 1992.

LEE, S.; MUNRO, M. Evaluation of in-plane shear test methods for advanced composite materials by the decision analysis technique. Composites, Elsevier, v. 17, n. 1, p. 13-22, 1986.

LEEUWEN, D. V. et al. Comparison of static shear test methodologies; test results and analysis. In: 2008 Beijing International Wind Energy Conference. [S.l.: s.n.], 2008. p. 29-31.

LEKHNITSKII, S. Theory of elasticity of an anisotropic elastic body. Hoden-Day, San Francisco, 1965.

LEMAITRE, J. A course on damage mechanics. [S.l.]: Springer Science \& Business Media, 2012.

LEMAITRE, J.; CHABOCHE, J.-L. Mécanique des matériaux solides. [S.l.]: Dunod Paris, 1985. v. 2.

LEMAITRE, J.; DESMORAT, R. Engineering damage mechanics: ductile, creep, fatigue and brittle failures. [S.l.]: Springer Science \& Business Media, 2005.

LIN, S.; WAAS, A. M. Experimental and numerical study on low velocity impact damage of a shear dominated composite laminate. In: AIAA Scitech 2019 Forum. [S.l.: s.n.], 2019. p. 1269.

LIU, D.; MALVERN, L. E. Matrix cracking in impacted glass/epoxy plates. Journal of Composite Materials, Sage Publications Sage CA: Thousand Oaks, CA, v. 21, n. 7, p. 594-609, 1987.

LOPES, C. et al. Multiscale simulation strategy for low-velocity impact on frp. In: Proc. Am. Soc. Compos.-29th Tech. Conf. Compos. Mater. [S.l.: s.n.], 2014.

LOVE, A. E. H. On the small free vibrations and deformation of a thin elastic shell. Philosophical Transactions of the Royal Society of London, The Royal Society London, n. 179, p. 491-546, 1888.

MATZENMILLER, A.; LUBLINER, J.; TAYLOR, R. A constitutive model for anisotropic damage in fiber-composites. Mechanics of materials, Elsevier, v. 20, n. 2, p. 125-152, 1995.

MEDEIROS, R. d. Development of a criterion for predicting residual strength of composite structures damaged by impact loading. 2016. Tese (Doutorado) Universidade de São Paulo, 2016. 
MEER, F. P. Van der. Mesolevel modeling of failure in composite laminates: constitutive, kinematic and algorithmic aspects. Archives of Computational Methods in Engineering, Springer, v. 19, n. 3, p. 381-425, 2012.

MINDLIN, R. D. Influence of rotatory inertia and shear on flexural motions of isotropic, elastic plates. J. appl. Mech., v. 18, p. 31-38, 1951.

MIOT, S. Rupture de structures composites stratifiées sous chargements statique et de fatigue. 2009. Tese (Doutorado) — Aix-Marseille 1, 2009.

MIOT, S.; HOCHARD, C.; LAHELLEC, N. A non-local criterion for modelling unbalanced woven ply laminates with stress concentrations. Composite Structures, Elsevier, v. 92, n. 7, p. 1574-1580, 2010.

MURAKAMI, S. Effect of cavity distribution in constitutive equations of creep and creep damage. In: EUROMECH Colloquium on Damage Mechanics, Cachan, France. [S.l.: s.n.], 1981.

NILSSON, E. Residual strength prediction of composite laminates containing impact damage. Interface, v. 6, n. 7, p. 8, 2005.

NIU, M. C.-Y. Composite airframe structures: practical design information and data. [S.l.]: Adaso Adastra Engineering Center, 1992.

OBERKAMPF, W. L.; ROY, C. J. Verification and validation in scientific computing. [S.l.]: Cambridge University Press, 2010.

OLSSON, R. Impact response of orthotropic composite plates predicted from a one-parameter differential equation. AIAA journal, v. 30, n. 6, p. 1587-1596, 1992.

Closed form prediction of peak load and delamination onset under small mass impact. Composite Structures, Elsevier, v. 59, n. 3, p. 341-349, 2003.

PINHO, S.; IANNUCCI, L.; ROBINSON, P. Physically-based failure models and criteria for laminated fibre-reinforced composites with emphasis on fibre kinking: Part i: Development. Composites Part A: Applied Science and Manufacturing, Elsevier, v. 37, n. 1, p. 63-73, 2006.

Physically based failure models and criteria for laminated fibre-reinforced composites with emphasis on fibre kinking. part ii: Fe implementation. Composites Part A: Applied Science and Manufacturing, Elsevier, v. 37, n. 5, p. 766-777, 2006.

PINHO, S. T. et al. Failure models and criteria for frp under-in-plane or three-dimensional stress states including shear non-linearity. 2005.

PUCK, A.; SCHÜRMANN, H. Failure analysis of frp laminates by means of physically based phenomenological models. In: Failure Criteria in Fibre-Reinforced-Polymer Composites. [S.1.]: Elsevier, 2004. p. 832-876.

RABOTNOV, Y. N. Creep rupture. In: Applied mechanics. [S.l.]: Springer, 1969. p. $342-349$.

REDDY, J. N. An introduction to the finite element method. New York, 1993. 
Mechanics of laminated composite plates and shells: theory and analysis. [S.l.]: CRC press, 2003.

REISSNER, E. The effect of transverse shear deformation on the bending of elastic plates. J. appl. Mech., p. A69-A77, 1945.

RIBEIRO, M. L. Damage and progressive failure analysis for aeronautic composite structures with curvature. 2013. Tese (Doutorado) - Universidade de São Paulo, 2013.

RIBEIRO, M. L. et al. Experimental and numerical dynamic analysis of laminate plates via carrera unified formulation. Composite Structures, Elsevier, v. 202, p. 1176-1185, 2018.

SANDHU, R. Nonlinear behavior of unidirectional and angle ply laminates. Journal of Aircraft, v. 13, n. 2, p. 104-111, 1976.

SAVITZKY, A.; GOLAY, M. J. Smoothing and differentiation of data by simplified least squares procedures. Analytical chemistry, ACS Publications, v. 36, n. 8, p. 1627-1639, 1964.

SEBAEY, T. et al. Damage resistance and damage tolerance of dispersed cfrp laminates: Design and optimization. Composite Structures, Elsevier, v. 95, p. 569-576, 2013.

SHI, Y.; PINNA, C.; SOUTIS, C. Modelling impact damage in composite laminates: a simulation of intra-and inter-laminar cracking. Composite Structures, Elsevier, v. 114, p. 10-19, 2014.

SHI, Y.; SWAIT, T.; SOUTIS, C. Modelling damage evolution in composite laminates subjected to low velocity impact. Composite Structures, Elsevier, v. 94, n. 9, p. 2902-2913, 2012.

SODEN, P.; HINTON, M.; KADDOUR, A. Biaxial test results for strength and deformation of a range of e-glass and carbon fibre reinforced composite laminates: failure exercise benchmark data. In: Failure Criteria in Fibre-Reinforced-Polymer Composites. [S.1.]: Elsevier, 2004. p. 52-96.

SOUZA, G. S. et al. Optimization of thin-walled composite beams considering static loadings. 2019.

SOUZA, G. S. C. Otimização genética de vigas de paredes finas compósitas: uma abordagem fenomenológica. 2017.

SPIVAK, M. Calculus on manifolds: a modern approach to classical theorems of advanced calculus. [S.l.]: Westview Press, 1971.

SUN, J.; LEE, K.; LEE, H. Comparison of implicit and explicit finite element methods for dynamic problems. Journal of Materials Processing Technology, Elsevier, v. 105, n. 1-2, p. 110-118, 2000.

TALREJA, R. Damage and fatigue in composites-a personal account. Composites Science and Technology, Elsevier, v. 68, n. 13, p. 2585-2591, 2008. 
THORSSON, S. I. Experimental and numerical investigation of fiber reinforced laminated composites subject to low-velocity impact. Ph. D. Thesis, 2017.

THORSSON, S. I. et al. Experimental investigation of composite laminates subject to low-velocity edge-on impact and compression after impact. Composite Structures, Elsevier, v. 186, p. 335-346, 2018.

THORSSON, S. I.; WAAS, A. M.; RASSAIAN, M. Low-velocity impact predictions of composite laminates using a continuum shell based modeling approach part a: Impact study. International Journal of Solids and Structures, Elsevier, v. 155, p. 185-200, 2018.

Numerical investigation of composite laminates subject to low-velocity edge-on impact and compression after impact. Composite Structures, Elsevier, v. 203, p. 648-658, 2018.

TITA, V. Contribuição ao estudo de danos e falhas progressivas em estruturas de material compósito polimérico. 2003. Tese (Doutorado) — University of São Paulo, 2003.

TITA, V.; CARVALHO, J. D.; VANDEPITTE, D. Failure analysis of low velocity impact on thin composite laminates: Experimental and numerical approaches. Composite Structures, Elsevier, v. 83, n. 4, p. 413-428, 2008.

TRAVESA, A. T. Simulation of delamination in composites under quasi-static and fatigue loading using cohesive zone models. [S.l.]: Universitat de Girona, 2006.

VINSON, J. R.; SIERAKOWSKI, R. L. The behavior of structures composed of composite materials. [S.1.]: Springer, 2006. v. 105.

WANG, Y.-L.; HAO, Q.-D.; OU, J.-P. Evaluation of in-plane shear test methods for composite material laminates. Journal of Chongqing University, , v. 6, n. 3, p. 221-226, 2007.

YIGIT, A. S.; CHRISTOFOROU, A. P. Limits of asymptotic solutions in low-velocity impact of composite plates. Composite Structures, Elsevier, v. 81, n. 4, p. 568-574, 2007. 

Appendix 



\section{APPENDIX A - COMPUTATIONAL INVESTIGATIONS OF QUASI-STATIC TESTS}

As discussed before, this Appendix is destined to the preliminary investigations that will allow shear-after-impact computational and experimental analyses to be carried out. Firstly, the computational verification of the finite element models for quasi-static and drop-weight tests in which confrontation with the previously experimental results of Tita (TITA, 2003) is performed. Also, it is verified if the UMAT and VUMAT subroutines were successfully implemented with ABAQUS. This approach is chosen simply because Ferreira's (FERREIRA, 2014) computational results were already validated with the experimental data obtained by Tita (TITA, 2003).

Here is important to define what does verification and validation means. Verification is understood as the investigative process with respect to the successful implementation of a numerical code and the accuracy of the obtained results, given the employed mathematical model. On the other hand, validation stands for the process of evaluation of the physical precision of the obtained results, comparing them with high-fidelity experimental data (OBERKAMPF; ROY, 2010).

Two study cases were chosen to perform the initial computational analyses. Those are tensile tests in $\left[0^{\circ}\right]_{5}$ and $\left[ \pm 45^{\circ}\right]_{4 s}$ specimens, experimentally characterized by Tita (TITA, 2003) and computational implemented (with Ribeiro's modified criterion) by Ferreira (FERREIRA, 2014). The material properties and strength values identified by Tita are exposed in Table 18.

Table 18: Tita's material properties and strength values

\begin{tabular}{lllr}
\hline \multicolumn{2}{c}{ Elastic properties } & \multicolumn{2}{c}{ Strength values } \\
\hline$E_{11}$ & $127 \mathrm{GPa}$ & $X_{T}$ & $1940 \mathrm{MPa}$ \\
$E_{22}$ & $10 \mathrm{GPa}$ & $X_{C}$ & $930 \mathrm{MPa}$ \\
$G_{12}$ & $5.4 \mathrm{GPa}$ & $Y_{T}$ & $47 \mathrm{MPa}$ \\
$G_{23}$ & $3.05 \mathrm{GPa}$ & $Y_{C}$ & $130 \mathrm{MPa}$ \\
$\nu_{12}$ & 0.34 & $S_{12}$ & $53 \mathrm{MPa}$ \\
$\nu_{23}$ & 0.306 & $S_{13}$ & $53 \mathrm{MPa}$ \\
$\rho_{c}$ & $1580 \mathrm{~kg} / \mathrm{m}^{3}$ & $S_{23}$ & $89 \mathrm{MPa}$ \\
\hline
\end{tabular}

Obs: $G_{12}=G_{13}$ and $\nu_{12}=\nu_{13}$

The finite element model mimics the characteristics of the $\left[0^{\circ}\right]_{5}$ coupon. It possess $15 \mathrm{~mm}$ width, $250 \mathrm{~mm}$ length and $0.9 \mathrm{~mm}$ thickness, meshed with structured S8R shell elements with 8 nodes, 6 DoF per node and reduced integration. Thus, each ply have 0.18 $\mathrm{mm}$ thickness. The boundary conditions are of encastre (i.e. no translations and rotations 
allowed) and predefined displacement in the $x$-direction of $2.0 \mathrm{~mm}$ in opposite ends of the specimen. The damage parameter $d_{1}$ was set to be equal to 0.99 for the case in which $\sigma_{11} \geq X_{T}$, representing the brittle failure of the laminate. Also, the same incrementation strategy employed in Ferreira's work to avoid convergence issues during the simulation was adopted here. Also, the associated material model parameter for this problem is simply $E_{11}=E_{11_{0}}$. The computational FE model, compiled along with the UMAT subroutine, obtained results are depicted in Figure 78.

Figure 78: Computational FE results for tension in $\left[0^{\circ}\right]_{5}$ laminate.

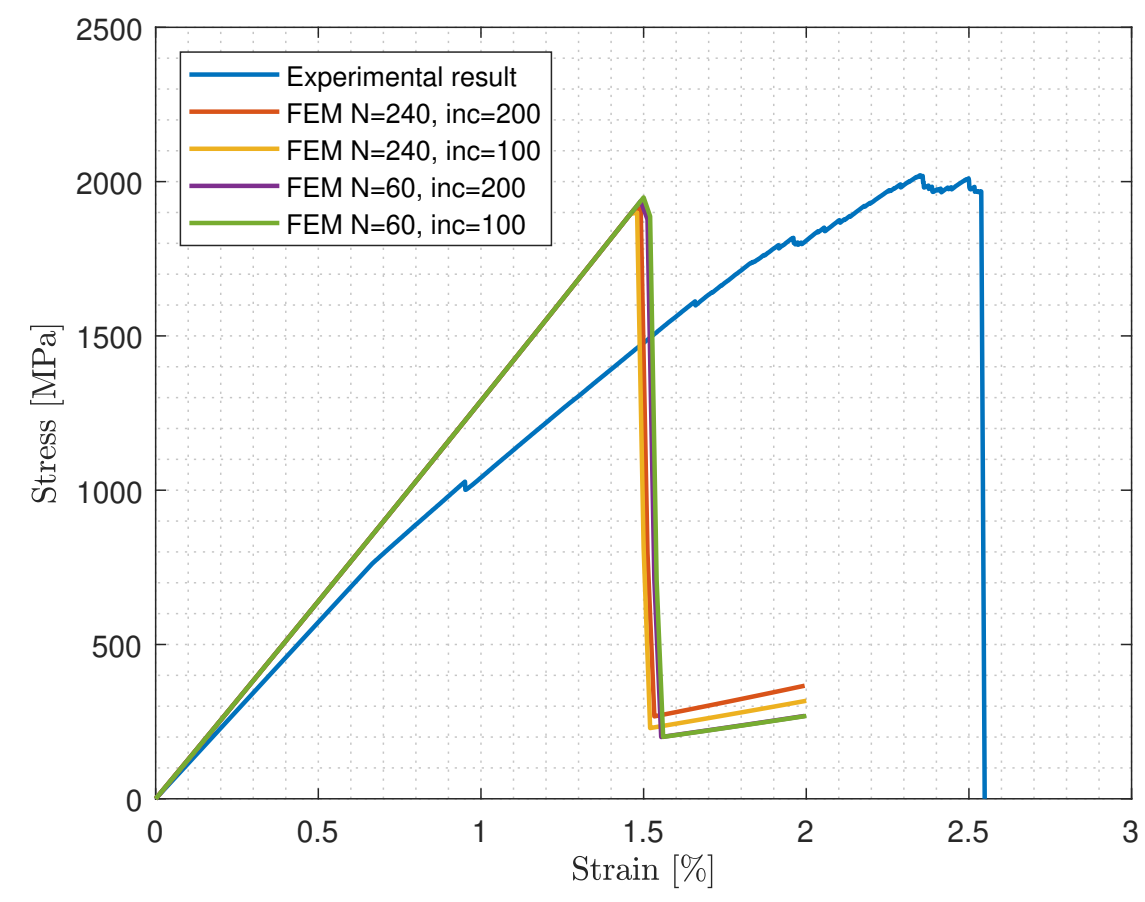

Source: by the author.

Firstly, it can be noticed that the FE results differs from the experimental one from Tita (TITA, 2003) specially for the maximum strain before failure. It is noteworthy to say that the stress-strain experimental curve presents change in slope with some sudden drops in the stress value probably due to the failure of some fibers during the test. On the other hand, Ferreira's model presented good maximum stress values when compared with Tita's result. Also, the sensitivity of the FE simulations with respect to the mesh density and number of increments is assessed. Figure 79 shows up a detailed cut of the stress-strain computational curve.

It can be noticed that the stress-strain curves possesses linear elastic behaviors with change in slopes for similar strain values. Although, for meshes with 60 elements this slope assumes negative values, while for finer meshes of 240 elements it assumes positive values. Also, this positive slope value physically represents the stiffness change due to 
Figure 79: Detailed cut of FE results for tension in $\left[0^{\circ}\right]_{5}$ laminate.

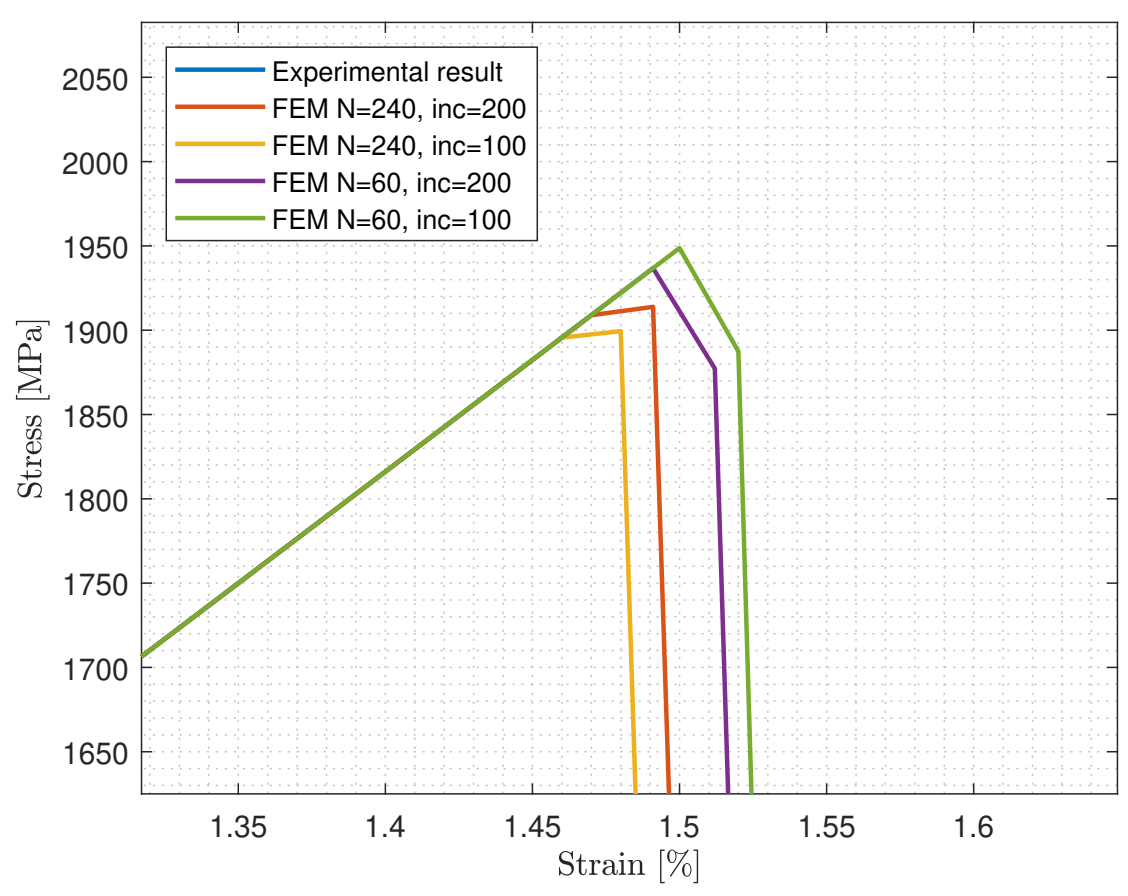

Source: by the author.

the ply damage in direction 1 . Moreover, all simulations presents very fast failure after damage detection/activation since the material behavior is brittle. Also, the FE models with 240 elements shows up capability of supporting increasing load after damage detection. Finally, all the simulations showed up that the material presents some residual strength to support loads after failure. This can be related to the experimental raw results, where after failure some fibers are still resisting to the applied load even though the ply have already suffered fracture. Still, it is notably that the change in the number of increments does not contaminate significantly the results. Quantitatively, Table 19 sums up the obtained results and compares them with the experimental one.

Table 19: Tensile results for $\left[0^{\circ}\right]_{5}$ coupon comparison

\begin{tabular}{|c|c|c|c|c|c|}
\hline Parameter & Tita (2003) & $\begin{array}{r}\text { FEM } \\
\mathrm{N}=240, \text { inc }=200\end{array}$ & $\begin{array}{r}\text { FEM } \\
\mathrm{N}=240, \text { inc }=100\end{array}$ & $\begin{array}{r}\text { FEM } \\
\mathrm{N}=60, \text { inc }=200\end{array}$ & $\begin{array}{r}\mathrm{FEM} \\
\mathrm{N}=60, \text { inc }=100\end{array}$ \\
\hline Stress before failure $X_{T}[\mathrm{MPa}]$ & 1940 & 1914 & 1899 & 1877 & 1887 \\
\hline Corresponding strain [\%] & 2.54 & 1.49 & 1.48 & 1.51 & 1.52 \\
\hline Damage detection stress [MPa] & $\mathrm{n} / \mathrm{a}$ & 1909 & 1896 & 1937 & 1949 \\
\hline
\end{tabular}

Regarding the experimental results of Tita, the computational obtained results of this work for 240 elements and 200 increments presents relative errors of $1.34 \%$ and $41.34 \%$ for the failure stress and the corresponding strain, respectively. Thus, Ferreira's model presented conservative results since the specimen failure occurred for lower values 
of strains.

By virtue of the great difference obtained for the ultimate strain value and due to documented stiffness drops in the stress-strain curves as a consequence of removing strain gages during the tests carried by Tita, the material model was tested with respect to the experimental results for the CFRP characterized in this work in Figure 44. The same simulation strategies (i.e. mesh density and number of increments) adopted for the previous analyses were employed for it. The obtained result is depicted in Figure 80.

Figure 80: Computational FE and experimental results for tension in $\left[0^{\circ}\right]_{8 T}$ laminate.

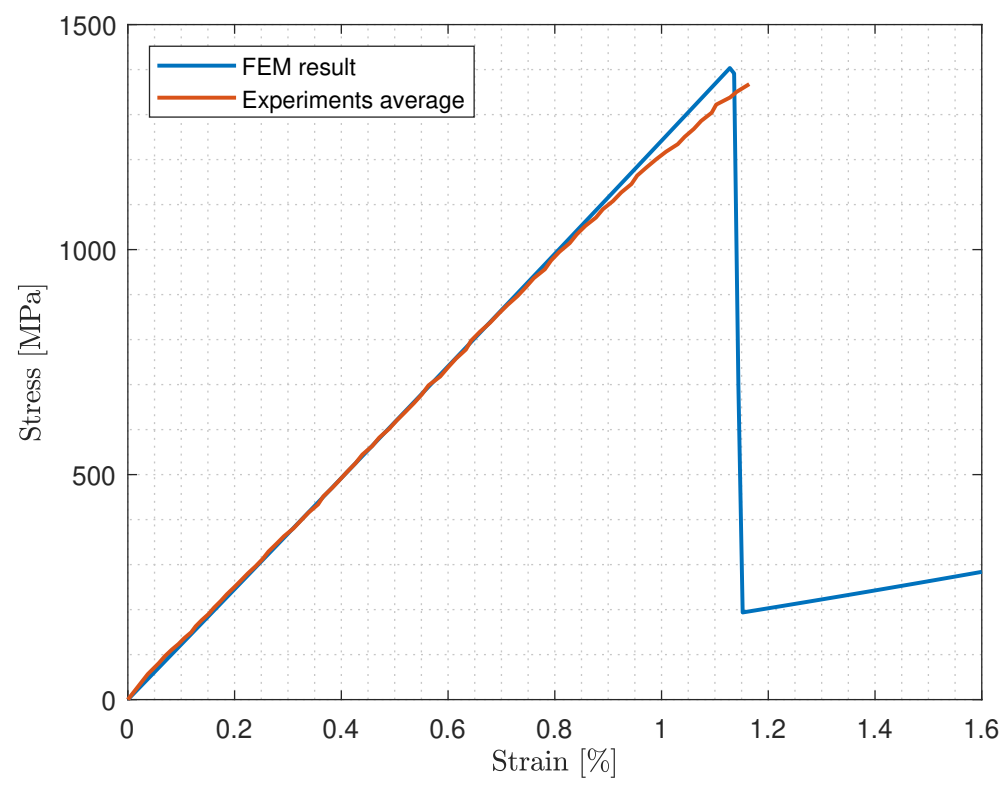

Source: by the author.

It can be observed that good agreement between the FE and experimental results were obtained. Table 20 shows up a comparative between the results.

Table 20: Tensile results for $\left[0^{\circ}\right]_{8 T}$ coupon comparison

\begin{tabular}{lrrr}
\hline Parameter & Experimental & FEM N=800 inc=200 & Relative error [\%] \\
\hline Stress before failure $X_{T}[\mathrm{MPa}]$ & 1404.8 & 1391.5 & 0.95 \\
Corresponding strain $[\%]$ & 1.18 & 1.14 & 3.51 \\
Damage detection stress $[\mathrm{MPa}]$ & $\mathrm{n} / \mathrm{a}$ & 1403 & $\mathrm{n} / \mathrm{a}$ \\
\hline
\end{tabular}

Regarding the experimental curve, it can be noticed that a non-linear behavior is detected somewhere around $1000 \mathrm{MPa}$ stress value, mathematically represented by a change in the slope of it. Again, the greater difference observed is in the ultimate strain value but, this time, it is distinct by a reasonable amount. This reinforce that the adopted strategy to obtain the strain field using the DIC technique is a good approach. Moreover, 
these results demonstrate that the implemented material model in this work captures with great accuracy the behavior of FRP laminates under tension in direction 1. Furthermore, the $\left[ \pm 45^{\circ}\right]_{4 s}$ specimen was also computationally implemented with the UMAT subroutine. It possess $250 \mathrm{~mm}$ length, $25 \mathrm{~mm}$ width and $2.7 \mathrm{~mm}$ thickness. Increment number, mesh density and element type used for its simulation were the same as the ones for the $\left[0^{\circ}\right]_{5}$ coupon. The boundary conditions were of encastre and predefined displacement of $4.5 \mathrm{~mm}$ at opposite faces. In addition, for this model the relevant damage variables are $d_{2}$ and $d_{6}$ with its respective evolution laws shown in Eq. (2.73). Also, the elliptical ply damage threshold limit of Eq. (2.59) proposed by Ferreira and illustrated in Figure 24 are relevant as well. Moreover, the material model parameters used are the same as the ones identified by Ferreira and the reference should be consulted for its assessment. Figure 81 shows a comparative between the FE model and Tita's experimental results.

Figure 81: Computational FE and experimental results for tension in $\left[ \pm 45^{\circ}\right]_{4 s}$ laminate.

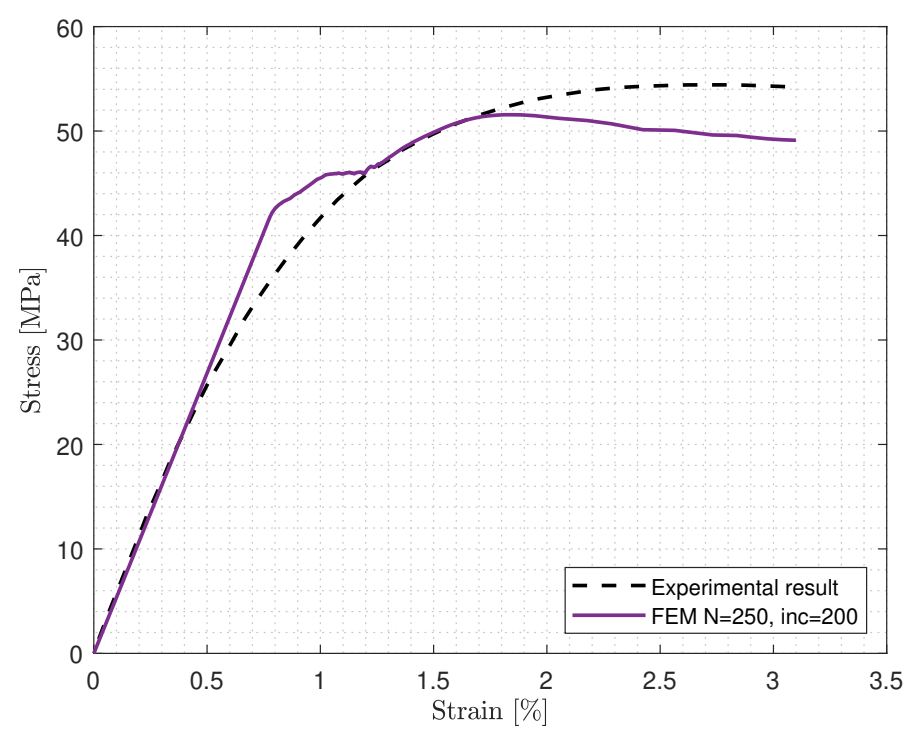

Source: by the author.

Some interesting features can be observed. The computational stress-strain curve presents a linear behavior at its first portion, followed by a non-linear one firstly detected for approximately $42 \mathrm{MPa}$ of stress as can be seen in the detailed cut of Figure 82, representing damage detection and a change in slope (i.e. reduction of $G_{12}$ ). Additionally, the FE model captures well the behavior of the laminate until $30 \mathrm{MPa}$ stress in which it diverges by a significant amount from the experimental curve. After the identification of ply damage, the FE curve presents a plateau of almost constant stress values $(\approx 46$ $\mathrm{MPa})$ for growing strain $(\approx 1.0-1.2 \%)$ until it reaches the experimental curve. Then, the FE model presents good agreement with the experiment, showing the same tendency and accuracy until $1.70 \%$ strain value. 
Figure 82: Detailed cut of obtained results for tension in $\left[ \pm 45^{\circ}\right]_{4 s}$ laminate.

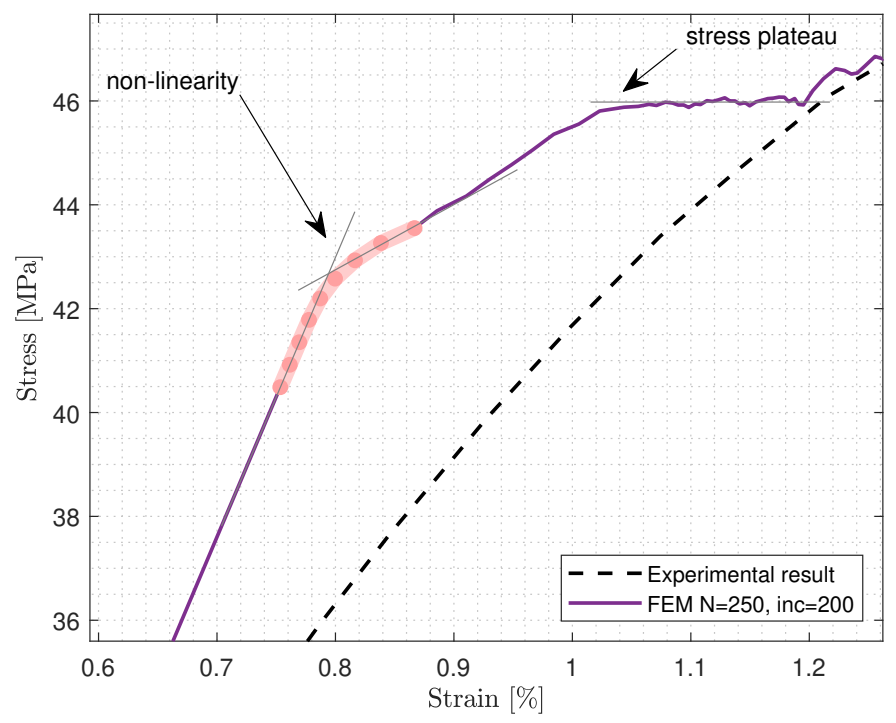

Source: by the author.

After this, the FE model smoothly diverges from the experimental results, although it possess similar failure stress values of $49.1 \mathrm{MPa}$ and $54.2 \mathrm{MPa}$ (relative error of $9.41 \%$ ) for the FE model and the experiment, respectively. Also, the oscillation pattern noticed by Ferreira is not here observed. All these features can be better observed while monitoring the damage variables values in Figure 83.

Figure 83: Damage variables values monitoring for $\left[ \pm 45^{\circ}\right]_{4 s}$ laminate.

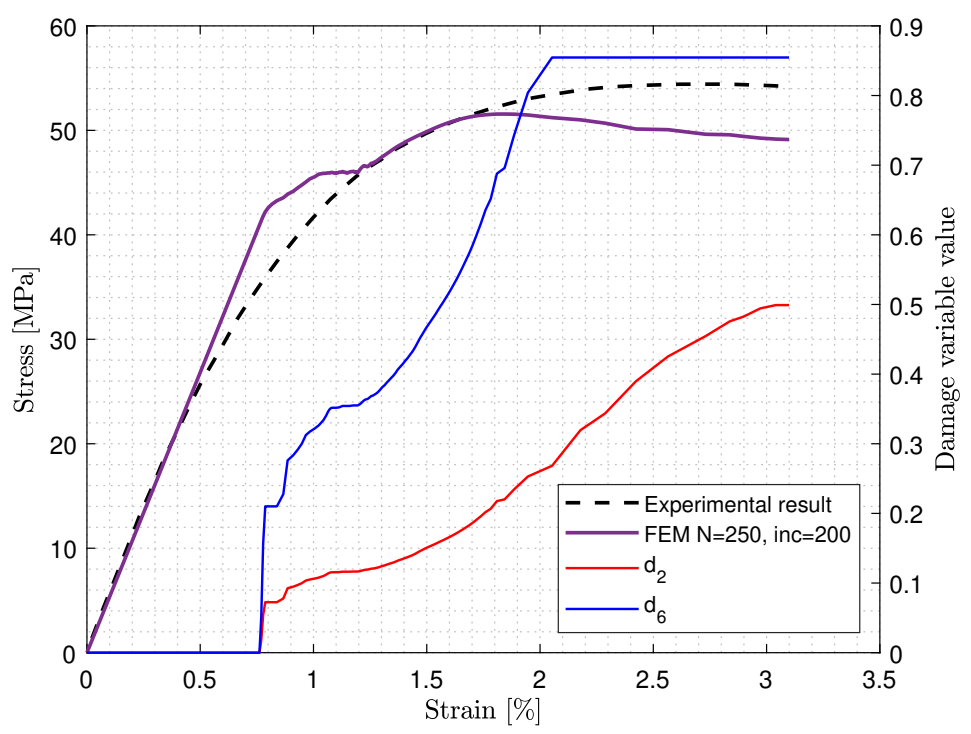

Source: by the author. 
It can be observed that the damage variable $d_{6}$ is the main responsible for the change of the behavior of the stress-strain curve from linear to non-linear. Also, the stress plateau observed is due to damage plateau values of both $d_{2}$ and $d_{6}$. Next, both values grows with $d_{6}$ still dominating the behavior until the stress sudden drop which is characterized by the abrupt growth of both damage variables that remains constant at the oscillatory portion of the stress-strain FE curve. It is worth to mention that these values were taken at one integration point of the first element to experience damage. Qualitatively, this behavior is captured by the FE simulation and depicted in Figures 84 and 85 .

In them, the behavior of the damage variables is assessed at the interest moments of damage detection (a), plateau (b) and sudden growth (c), respectively. Moreover, the material model showed that, regarding to some of the reported issues, it can model the behavior of these laminates. 
Figure 84: Damage variables monitoring: $d_{2}$.
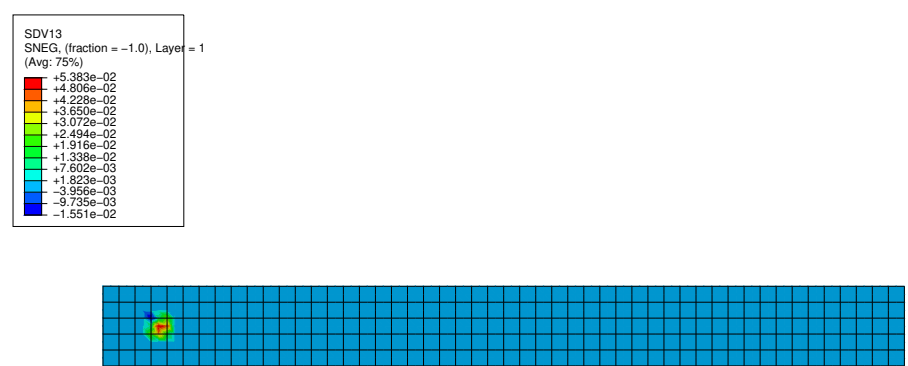

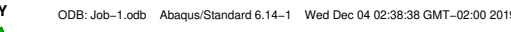

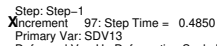

Deformed Var: U Deformation Scale Factor: $+1.000++0$

(a)
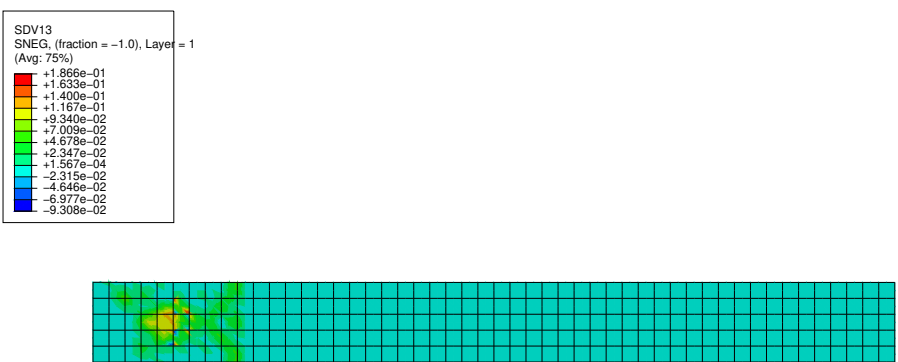

Y ODB: Job-1.odb Abaqus/Standard 6.14-1 Wed Dec 04 02:38:38 GMT-02:00 2019

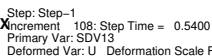

(b)
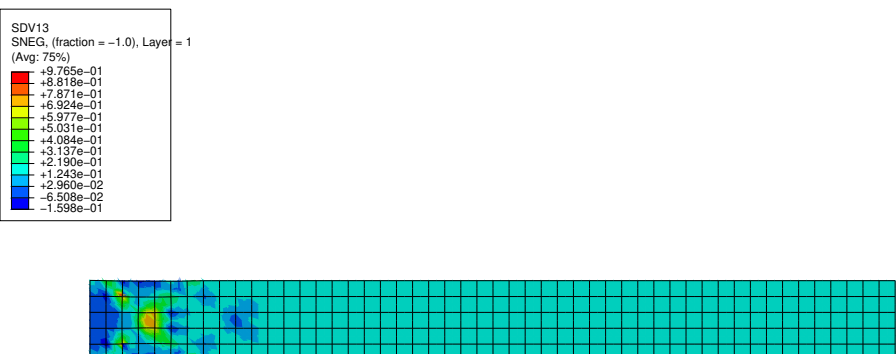

ODB: Job-1.odb Abaqus/Standard 6.14-1 Wed Dec 04 02:38:38 GMT-02:00 2019

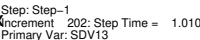

Primary Var: SDV13

(c)

Source: by the author. 
Figure 85: Damage variables monitoring: $d_{6}$.
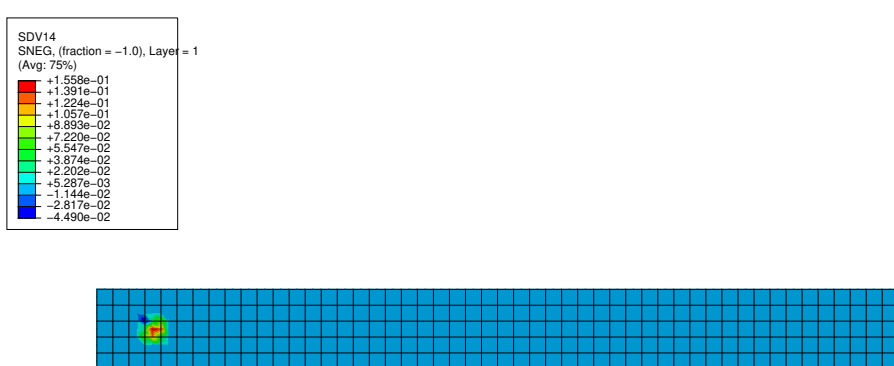

Y ODB: Job-1.odb Abaqus/Standard 6.14-1 Wed Dec 04 02:38:38 GMT 020020019

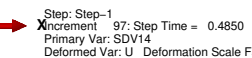

(a)
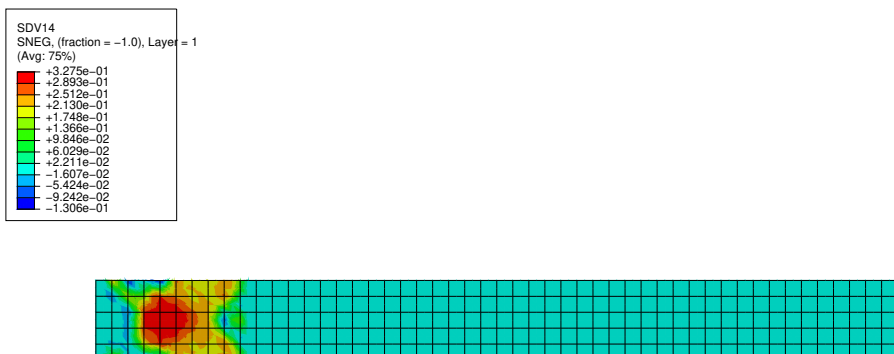

Y ODB: Job-1.0db Abaqusistandard 6.14-1 Wed Dec 04 02:38:38 GMT-02:00 2019

$\longrightarrow \begin{gathered}\text { Step: Step- } 1 \\ \text { Primemant } 108: \text { Step Time }\end{gathered}=0.5400$

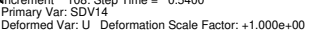

(b)
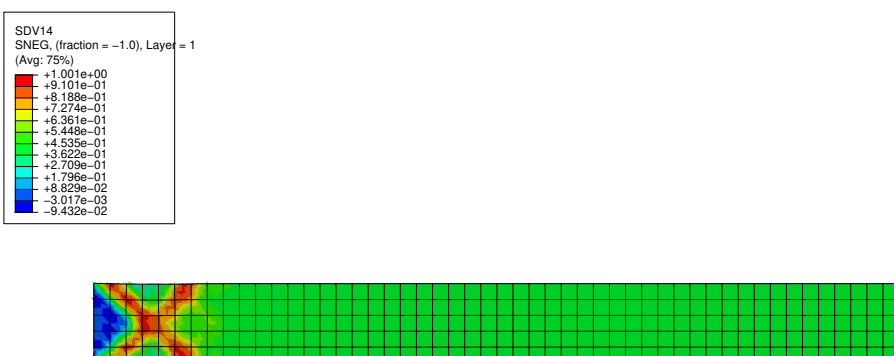

Y ODB: Job-1.odb Abaqus/Standard 6.14-1 Wed Dec 04 02:38:38 GMT 02:00 2019

Step: Step-1
Xncrement 202: Step Time $=1.010$
Primary Var: SDV14

Xncrement 202: Step Time
Primary Var: SDV 14
Detory

(c)

Source: by the author. 



\section{APPENDIX B - DROP-TOWER CALIBRATION}

An aluminum 7075-T6 metallic plate was chosen to be subjected under an impact event to assess the influence of the boundary conditions in the computational model of the drop-test. Thus, following the experimental-computational strategy already described, confrontation of the obtained results is carried out. The test parameters are described in Table 21 and were chosen in this fashion so that only the residual plastic strains in the plate are responsible for the absorbed energy level during the impact. Plate dimensions are of 150x100 mm weighting 53.6g.

Table 21: Drop-test parameters for Al 7075-T6

\begin{tabular}{|c|c|c|c|c|}
\hline Impactor mass $[\mathrm{kg}]$ & Test height $[\mathrm{m}]$ & Initial velocity $[\mathrm{m} / \mathrm{s}]$ & Total impact energy $[\mathrm{J}]$ & Impactor radius [mm] \\
\hline 4.83 & 0.117 & 1.51 & 5.525 & 8.0 \\
\hline \multicolumn{4}{|c|}{ Impactor material } & Aluminum \\
\hline \multicolumn{4}{|c|}{ Plate thickness $[\mathrm{mm}]$} & 1.5 \\
\hline \multicolumn{4}{|c|}{ Gravitational acceleration $\left[\mathrm{m} / \mathrm{s}^{2}\right]$} & 9.7849 \\
\hline
\end{tabular}

Also, for obtaining the total impact energy, the local gravitational acceleration is calculated considering Helmert's equation,

$$
g(\phi)=9.780327 \times\left[1.0026454-0.0026512 \cos (2 \phi)+0.0000058 \cos ^{2}(2 \phi)\right]
$$

in which $\phi$ is the latitude of São Carlos city (São Paulo, Brazil) where the tests were performed.

Firstly, the sample acquisition rate of the LabAmp equipment needs to be set. For example, for an impact duration of $4 \mathrm{~ms}$ and with $200 \mathrm{kSps}$ (kilo samples per second), the equipment will capture up to 800 data points for the force and displacement histories. Thus, for impact events with low duration, high sampling acquisition rate is required to capture the smallest possible tendencies in these curves and, consequently, the target's behavior. In this sense, for the drop-weight test in the aluminum plate the sampling rate was set in $50 \mathrm{kSps}$. Figure 86 shows up the force history.

It can be noticed high-frequency oscillations in the initial portion of the curve (up to approximately $0.8 \mathrm{~ms}$ ) due to the accommodation of the impactor with the plate. Some of these oscillations persist until $1.4 \mathrm{~ms}$ but with lower amplitude. Moreover, it can be seen that the curve is smooth in some sense, thus, since no more high-level (frequency and/or amplitude) oscillations are noticed it can be stated that no damage was introduced in the plate (i.e. there was no cracks onset and propagation) during the impact event. 
Figure 86: Force history of the drop-test for the Al 7075-T6 plate.

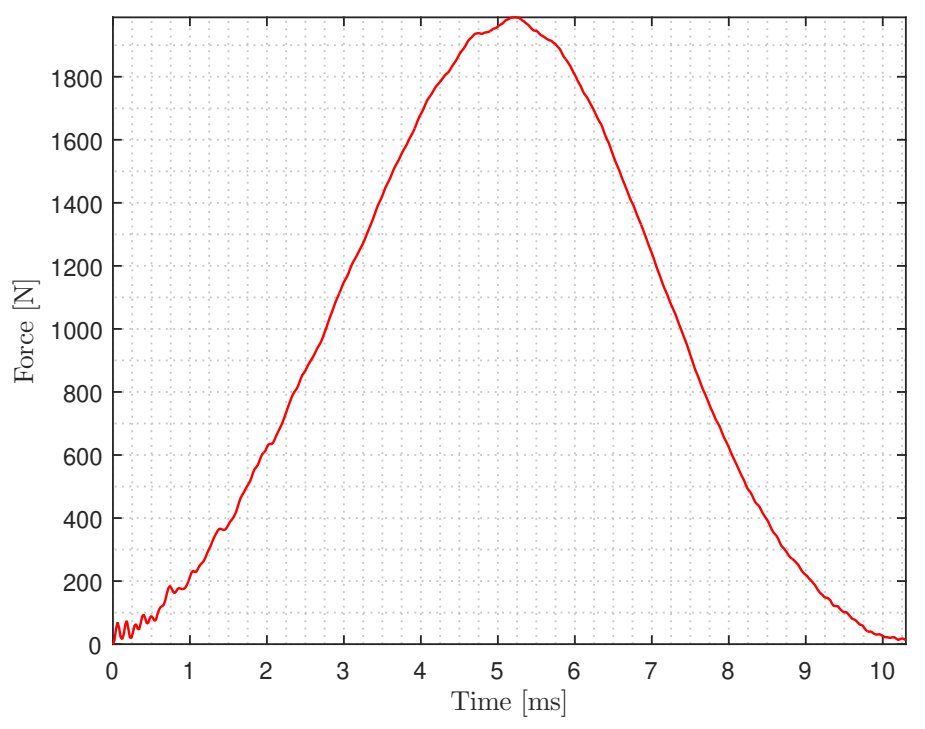

Source: by the author.

Regarding to the displacement history some caution needs to be take. Since the LDS system needs a plane and white surface for its well-functioning, a T-beam is coupled in the drop-tower frame with this purpose (Figure 87).

Figure 87: Drop-tower frame with the white paint sprayed T-beam (circled).

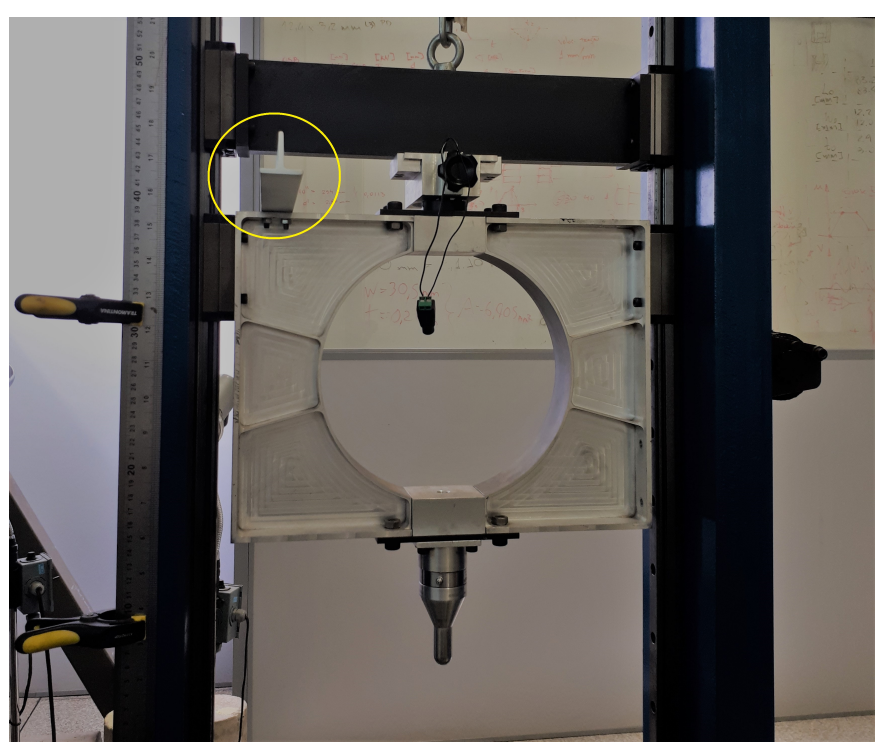

Source: by the author.

Then, a Mitutoyo magnetic base with the LDS attached to it is assembled in the lateral beams of the drop-tower and the laser is pointed to the white T-beam for displacement measurement. Also, the physical quantity that is captured is voltage and 
provided with the LDS calibration curve it is converted to distance. Moreover, there is still necessity to be taken into account the difference between the distances of the beam and impactor heights. Thus, the conversion is given as,

$$
u_{i}(t)=-2.5\left(V_{i}(t)-V_{0}\right)
$$

where $u_{i}$ is the displacement for the $i$-th data (in millimeters), $V_{i}$ is the measured voltage for the same data point and $V_{0}$ is the reference measured voltage taken as the one captured at the moment of contact between the impactor and the target (both in volts). The minus sign in the right hand-side of Eq. (B.2) is introduced to make the displacement positive. Finally, the displacement is plotted against time as presented in Figure 88.

Figure 88: Displacement history of the drop-test for the Al 7075-T6 plate.

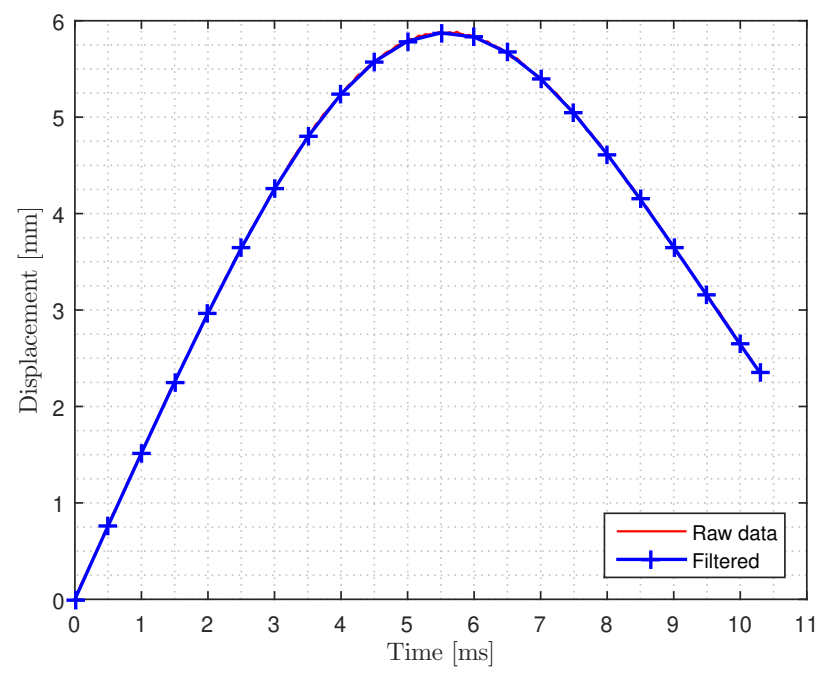

Source: by the author.

From Figure 88, it can be noticed two features in contrast with the force history of Figure 86, listed as follows:

1. The displacement history does not return to zero: this is simply because the LDS keeps capturing voltage (or distance) measures even though the impactor is not in contact with the target anymore (i.e. there is no more force measurement);

2. There is the raw and the filtered data curves: this is done because of the precision of the acquirement of the LDS and is going to be explained when the energy history is presented.

Moreover, no high-frequency oscillations are readily noticed in the displacement history. Provided with the force and displacement histories a force-displacement curve can be produced. Figure 89 presents it, plotted with reference to the raw data of displacement. 
Figure 89: Force-displacement curve of the drop-test for the Al 7075-T6 plate.

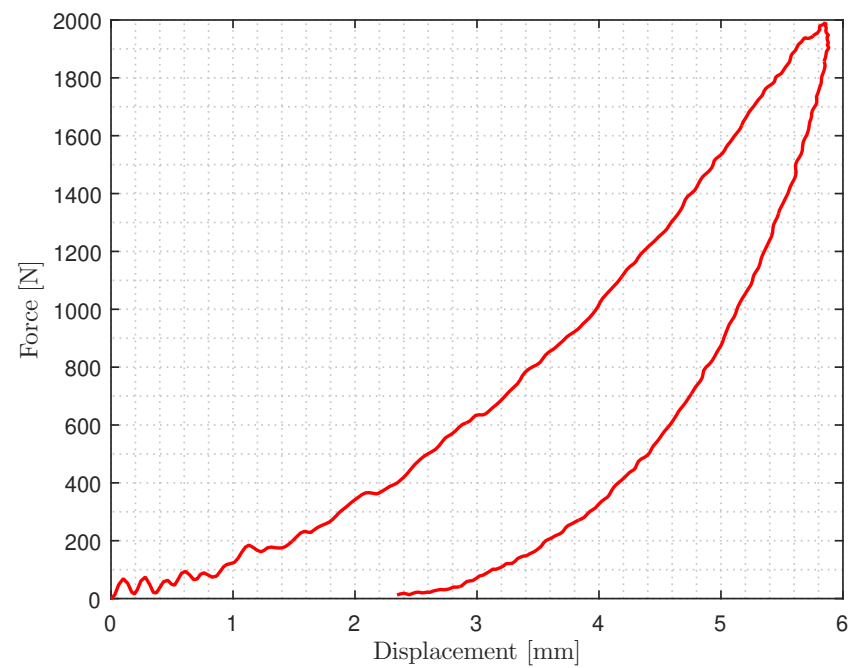

Source: by the author.

In the curve a permanent indentation of the plate is accused. Also, during the drop-test this feature is noticed. Since the displacement is measured with respect to the impactor and not to the plate directly, the residual displacement (i.e. plastic strain) in Figure 89 is only an approximation of the real value. Nevertheless, it can be noticed that the permanent deformation is approximately $40 \%$ of the maximum displacement experienced by the aluminum plate.

Regarding to the energy history, some data treatment need to be performed before its plotting. The numeric integration of the force history approach done by Tita (TITA, 2003; TITA; CARVALHO; VANDEPITTE, 2008) to obtain the instant velocity and, posteriorly, the energy history provided unrealistic results; it accused that the transferred kinetic energy returns to zero meaning that no energy absorption occurred during the impact event which is not true. Thus, a numeric differentiation approach was employed although it is naturally a less accurate method than the last one. Using the central difference approximation method for the first derivative of the displacement, it follows that the velocity is given as,

$$
v(t)=\frac{u(t+h)-u(t-h)}{2 h}+\mathcal{O}\left(h^{2}\right),
$$

where $h=\left(t_{n}-t_{0}\right) / n$ is the adopted time step with $n$ the number of data points, $t_{n}$ and $t_{0}$ the final and initial analysis times, respectively. Figure 90 shows up the obtained instant velocity for the differentiation performed with raw and filtered data.

It can be noticed good agreement in the tendency of the curves. Both starts with the initial velocity value, pass through zero at the point of maximum displacement and then reaches negative values indicating a slope change in the displacement. All this discussion 
Figure 90: Instant velocity of the drop-test for the Al 7075-T6 plate.

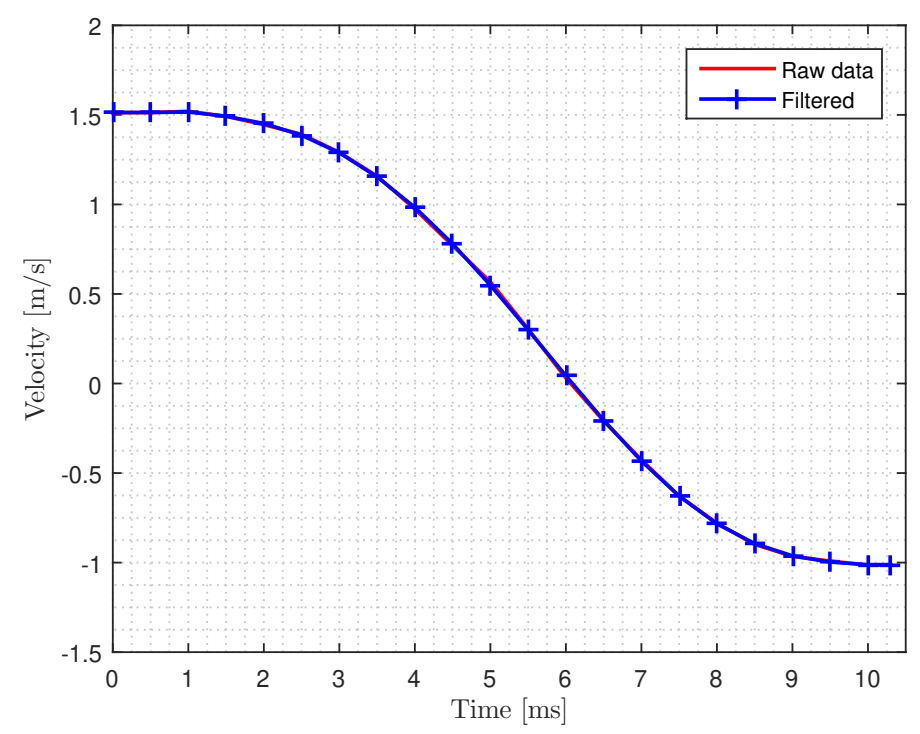

Source: by the author.

is done with the objective of justifying the hereafter employed strategy for obtaining the energy history. With the raised raw data during the drop-tests, if one tries to plot this curve, the obtained result will be the one depicted in Figure 91.

Figure 91: Energy history of the drop-test for the Al 7075-T6 plate (complete raw data).

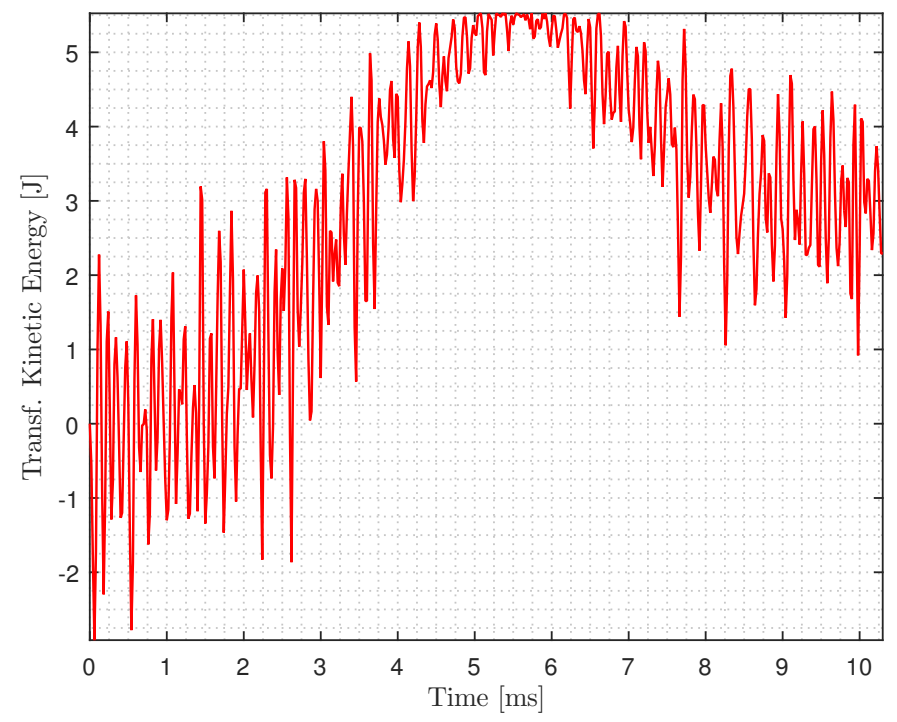

Source: by the author.

In this, all data points are included. This result encourages the appliance of a filter on the displacement data for well-capturing the energy history. Therefore, a Savitzky-Golay 
(S-G) filter (SAVITZKY; GOLAY, 1964) was applied in the displacement curve to smooth the data, increasing the precision of it without distorting its tendency. Further, the equally spaced subsets of $2 m+1$ odd data points are fitted to a polynomial of degree $p \leq 2 m$ in the least squares sense. This filter is native in MATLAB (sgolayfilt), where the data treatment was performed. The inputs for the filter are the points to be smoothed, the polynomial order and the frame length that must be odd. After some try outs, it was noticed that the relative error between the displacement of the raw and filtered data points varies with the number of points taken for the filtering (and, consequently, with the frame length) and the polynomial order chosen. Therefore, an optimization based on genetic algorithms (GA) was performed with the objective of minimizing the relative error and maximizing the correlation coefficient between the raw and filtered data. At this point it is important to say that the curves depicted in Figures 88 and 90 were already shown with the optimal parameters found by the GA. The used genetic algorithm toolbox was previously developed in the work of Colherinhas (COLHERINHAS, 2016). In the GA, populations of individuals (or chromosomes) are randomly generated and evaluated by a fitness function that rank them according to one or more criteria. Then, those chromosomes are submitted to selection mechanisms and genetic operators such as cross-over and mutation to prosper through generations (SOUZA et al., 2019). This GA toolbox is real value codified to reduce computational cost during its execution. Also, it makes use of uniform creep mutation that locally explores the search space by the hands of little perturbations on the genes (multiplies one of them by a value close to unity) and blend cross-over (BLX- $\alpha$ ) enlarging the father's crossing region which raises the genetic variability. Finally, elitism and decimation mechanisms takes place. These are deterministic types of genetic operators that are used to preserve and remove individuals with high and low values of fitness values, respectively. After the initial population is generated and the chromosomes evaluated by the fitness function, some of the individuals needs to perpetuate to the next generation. This is performed by a stochastic selection strategy known as the roulette-wheel method. In it, an imaginary roulette will arbitrarily select individuals based on the cumulative probability given as,

$$
q_{i}=\sum_{k=1}^{i} P_{k}
$$

where $P_{k}$ is the probability that one individual has of being chosen. The last is,

$$
P_{i}=f(i) / \sum_{i=1}^{N} f(i)
$$

with $N$ being the number of chromosomes in the population. The parameters used through the optimization process are depicted in Table 22.

These parameters were chosen after several tr yout executions of the algorithm providing good convergence characteristics, cost-benefit computational cost and quality 
Table 22: Optimization parameters

\begin{tabular}{ccr}
\hline Parameter & Methodology & Value \\
\hline Number of generations & - & 100 \\
Number of chromosomes per generation & - & 50 \\
Decimation step & - & 20 \\
Decimation percentage & Deterministic & $20 \%$ \\
Elitism percentage & Deterministic & $2 \%$ \\
Mutation probability & Uniform creep & $2 \%$ \\
Cross-over probability & BLX- $\alpha$ & $60 \%$ \\
\hline
\end{tabular}

of results of the GA. The problem constraints were chosen in the limiting intervals $1 \leq$ order $\leq 10$ and $1 \leq n_{p} \leq 100$ where $n_{p}$ is defined as the step in which the acquired data is considered for analysis, i.e. $u\left(1: n_{p}: n\right)$ with $n$ the number of measured points, and order is the polynomial order used in S-G filter. These limits were chosen so that reasonable results are encountered. Aiming the maximum relative error $e_{r}$ minimization and correlation coefficient $R$ maximization and having in mind that both of these values ranges from zero to unity, the fitness function is defined so that a normalization of its values are achieved. Thus, it follows that,

$$
f_{\text {obj }}=\max \left(R^{e_{r}}\right)
$$

where $f_{\text {obj }}$ is the objective function. Thus, it follows that $f_{\text {obj }} \rightarrow 0$ if $R \rightarrow 0$ (independently of the $e_{r}$ value) and $f_{\text {obj }} \rightarrow 1$ if $e_{r} \rightarrow 1$ depending on the $R$-value. The last is simply,

$$
e_{r}=\left|1-u_{i}^{f} / u_{i}^{r}\right|
$$

in which $u_{i}^{f}$ and $u_{i}^{r}$ are the $i$-th displacement filtered and raw data points, respectively. The obtained optimization convergence curve of the problem is shown in Figure 92a.

In can be noticed that with few generations the GA provided high fitness values for the objective function. Also, there was a generation in which the medium fitness of the individuals were all closely to unity, showing that the adopted strategy provides good convergence. The optimal values found were of order $=9$ and $n_{p}=25$, resulting in a frame length of 22 data points. The relative error considering these values is shown in Figure 92b.

As can be seen, low error values were found. As a matter of fact, the maximum value is of $0.52 \%$ at the initial portion of the impact event reinforcing the initial "gross behavior" of the velocity curve in Figure 90. Regarding to the correlation coefficient, its value is of 0.9998 providing a fitness value of almost 1.0. Finally, the energy history for the drop-weight test is shown in Figure 93.

Again, in it the frame length was of 25 points, with the S-G filter order of 9 and the propagating gross behavior in the initial portion of the impact event. Nevertheless, it 
Figure 92: Optimization convergence curve (a) and relative error between raw and filtered displacement data (b).

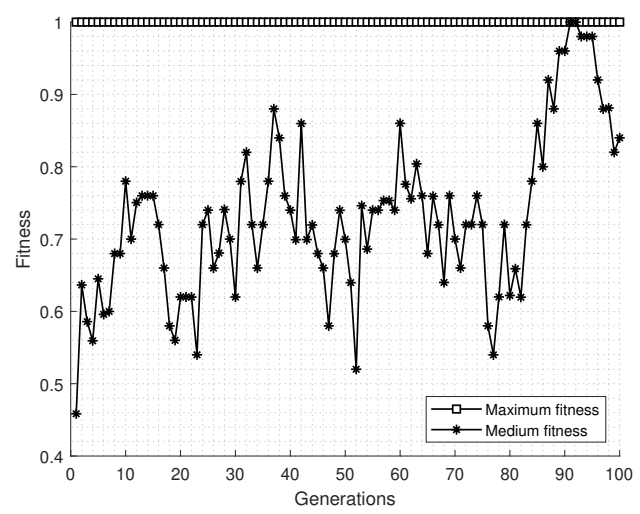

(a)

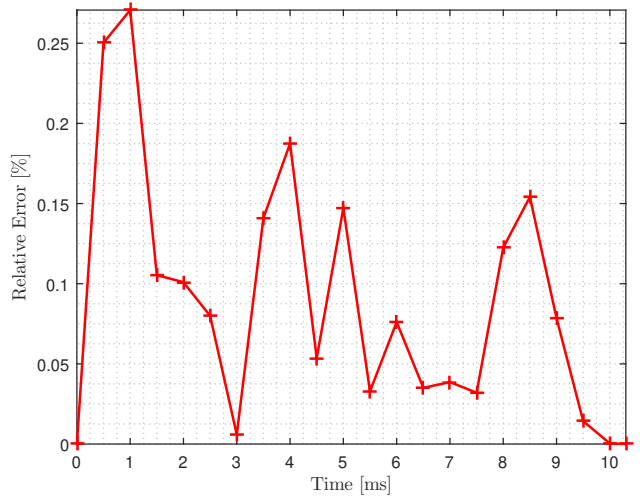

(b)

Source: by the author.

Figure 93: Energy history of the drop-test for the Al 7075-T6 plate.

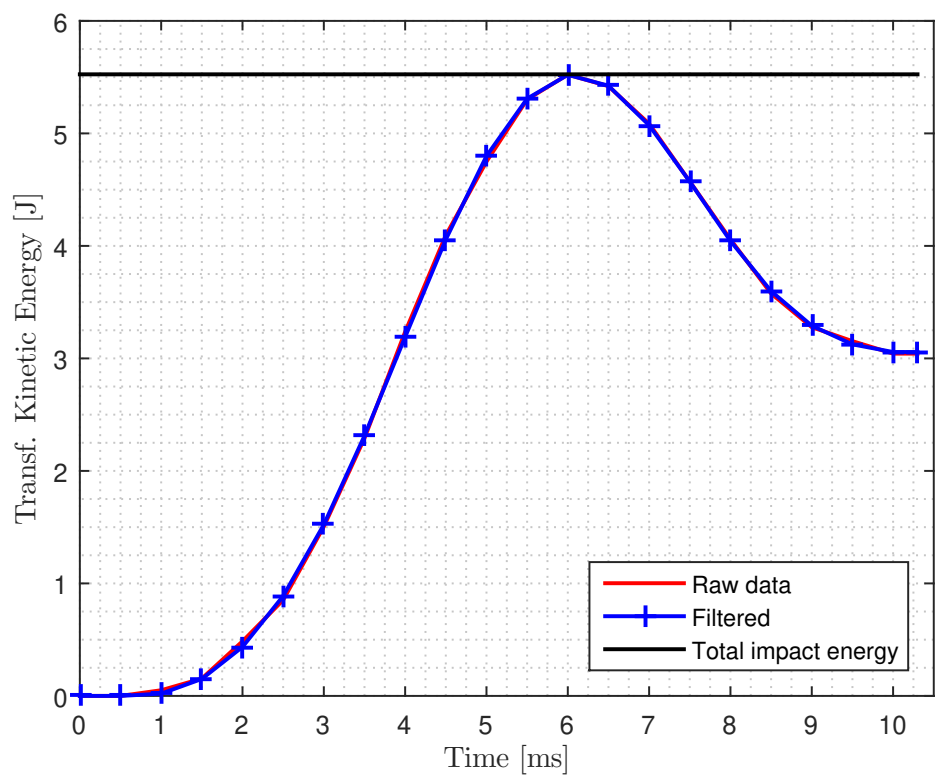

Source: by the author.

can be seen that it is physically consistent: the transferred kinetic energy starts from zero, scale to the total impact energy level and drops to the value of the absorbed energy that, for this drop-test, represents the greater portion of the total energy. This optimization strategy for obtaining the energy history by smoothing the data of measured displacement by the LDS is hereafter employed for the upcoming drop-tests in CFRP plates. In short, the procedure used in this work to obtain the energy history is shown in Figure 94. 
Figure 94: Energy history acquirement procedure.

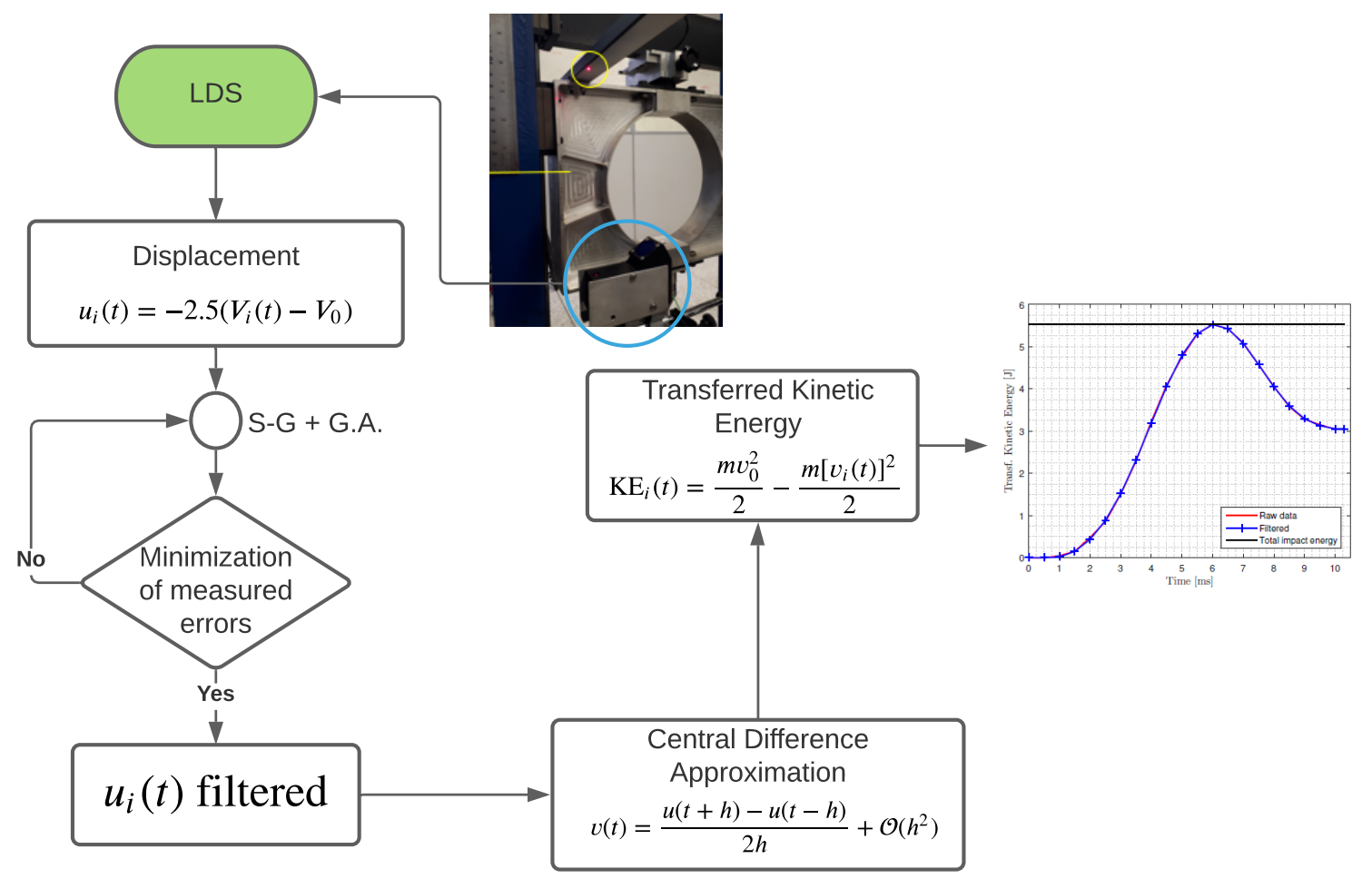

Source: by the author.

Regarding to some quantitative results, table 23 sums up the obtained outcomes of the drop-weight test in the aluminum plate.

Table 23: Aluminum plate drop-test summary of results

\begin{tabular}{lrr}
\hline Physical quantity & Value & Unit \\
\hline Impact duration & 10.3 & $\mathrm{~ms}$ \\
Peak force & 1988.5 & $\mathrm{~N}$ \\
Maximum displacement & 5.88 & $\mathrm{~mm}$ \\
Indentation & 2.35 & $\mathrm{~mm}$ \\
Elastic energy & 2.49 & $\mathrm{~J}$ \\
Absorbed energy & 3.04 & $\mathrm{~J}$ \\
\hline
\end{tabular}

Lastly, it is worth to mention that the absorbed energy represents around $55.07 \%$ of the total impact energy and, considering the approximation for the plate residual displacement (indentation), it follows that the elastic displacement value is of $3.53 \mathrm{~mm}$. 